\title{
OPTICALLY ACTIVE DYE-BASED SYSTEMS TEMPLATED BY DNA EXHIBITING EXCITONIC DELOCALIZATION
}

\author{
by \\ Brittany Lynn Cannon
}

\author{
A dissertation \\ submitted in partial fulfillment \\ of the requirements for the degree of \\ Doctor of Philosophy in Materials Science and Engineering \\ Boise State University
}

May 2018 
(C) 2018

Brittany Lynn Cannon

ALL RIGHTS RESERVED 


\section{BOISE STATE UNIVERSITY GRADUATE COLLEGE}

\section{DEFENSE COMMITTEE AND FINAL READING APPROVALS}

of the dissertation submitted by

Brittany Lynn Cannon

Dissertation Title: Optically Active Dye-Based Systems Templated by DNA Exhibiting Excitonic Delocalization

Date of Final Oral Examination: $\quad 22$ February 2018

The following individuals read and discussed the dissertation submitted by student Brittany Lynn Cannon, and they evaluated her presentation and response to questions during the final oral examination. They found that the student passed the final oral examination.

William B. Knowlton, Ph.D.

Bernard Yurke, Ph.D.

Paul H. Davis, Ph.D.

Paul W.K. Rothemund, Ph.D.
Co-Chair, Supervisory Committee

Co-Chair, Supervisory Committee

Member, Supervisory Committee

Member, Supervisory Committee

The final reading approval of the dissertation was granted by William B. Knowlton, Ph.D., Co-Chair of the Supervisory Committee. The dissertation was approved by the Graduate College. 


\section{DEDICATION}

This dissertation is dedicated to my mother and to my wonderful husband.

Mom, through the years you have raised me to become an independent and successful woman. I hope that this will act as proof to your success as a mother. I couldn't have been blessed with a better mentor and friend to support me through this rollercoaster of life.

Thank you for all that you do.

"If there ever comes a day when we can't be together, keep me in your heart. I'll stay there forever."

$\sim$ Winnie the Pooh

Colin, your infinite love and encouragement have given me the motivation and perseverance to push through the difficult times. I owe much of my success to you. I look forward to our (hopefully) long life together.

"Every love story is beautiful, but ours is my favorite."

$\sim$ Anonymous 


\section{ACKNOWLEDGEMENTS}

Through my few years at Boise State University, there have been many individuals and groups that have aided in my success. I am grateful for every piece of advice and all words of encouragement that I have been provided with. Without such an amazing support group, the completion of this dissertation would not have been possible. I would like to specifically acknowledge a few individuals who have significantly influenced my experience:

I would like to begin by thanking my advisors: Dr. William Knowlton and Dr. Bernard Yurke. Dr. Knowlton has gone above and beyond as an adviser and mentor. Most notably, Dr. Knowlton has had an impactful influence on both my academic career and personal life, which has probably gone under appreciated. I can only hope that the culmination of this work will do justice to the mentorship that he has provided me with. Dr. Yurke has given me astounding confidence in my work through our long and somewhat tedious discussions that, more often than not, lead to long mathematical derivations. The theoretical background that he has provided me with will (without a doubt) help immensely with my future career. Additionally, I would like to extend the utmost gratitude to my committee members Drs. Paul Davis and Paul Rothemund. Both have been instrumental in the successful progression of my dissertation. Their advice was appreciated more than I could ever reciprocate. 
I am grateful for Dr. Elton Graugnard, Dr. Wan Kuang, Dr. William Hughes, Dr. Lizandra Godwin, Dr. Reza Zadagen, Dr. Jennifer Padilla, and Dr. Natalya Hallstrom for all of their support, encouragement, and research advice.

I am appreciative of the comradery that I have developed amongst the fellow students. Particularly, I would like to thank Donald Kellis and Lance Patten for their aid in the laboratory and overall emotional support. I am also grateful to Brett Ward, Michael Tobiason, Sadao Takabayashi, and Shohei Kotani for bringing light and sarcasm into my life.

Finally, I would like to extend my appreciation to the Materials Science staff who made the magic happen, specifically, Chad Watson, Jessica Economy, Stephanie Moran, Jamie Hayward, and Krystal Holaday. Without their guidance, I would have been lost in a sea of forms. 


\begin{abstract}
The concept of quantum computing was first developed in the early 1980's. The attraction of quantum computers is their potential capacity to solve extremely complex problems, such as factorization, on a timescale far faster than that of classical computers. However, realization of quantum computation is currently in its infancy, and recent implementations possess serious drawbacks that reduce their appeal. Some challenges of current designs include the necessity to cool the systems using liquid helium to near absolute zero temperatures $(15 \mathrm{mK})$ in order to maintain sufficiently long-lifetimes of the Qbits (i.e., unit of quantum information), difficulty with scaling up the processing systems, and prohibitively high manufacturing costs.

Fundamentally, the key physical effect that enables high processing speeds in quantum computers is quantum superposition, which allows a single qbit to have two (or more) definite states (e.g., 0 and 1) simultaneously. Maintaining a superposition of states at room temperature, however, has proven difficult with silicon-based technology. Coherent exciton delocalization, which involves the superposition of excitonic states characterized by the delocalization of excitons (i.e., electron-hole pairs) across spatially proximate but separated molecules, has been observed in biological photosynthetic systems at ambient temperatures (295 K). Natural photosynthetic systems are composed of protein scaffolds that encompass and elegantly arrange an aggregate of optically active dye molecules (i.e., cluster of chromophores) with nanometer-scale precision in a manner that promotes coherence despite the inherently warm and "noisy" (i.e., rapidly
\end{abstract}


fluctuating) environment inside a plant. As a result, light energy absorbed from the sun is quickly and efficiently transferred through the dye aggregate in a wavelike manner that both optimizes the transfer pathway and minimizes energy loss. Thus, exploiting excitonic delocalization, as inspired by biology, offers a potential path forward towards realizing quantum computing at room temperature.

Here, we demonstrate coherent exciton delocalization in systems that utilize DNA, a biological material that affords atomically precise arrangement of dyes (e.g., Cy5) with nanometer proximity, as a scaffold. Leveraging the inherent programmability and functionality of DNA, which undergoes Watson-Crick base-pairing to enable simple structural control than the complex folding mechanisms involved with proteins, we have designed two dye-DNA complexes that are described in two journal manuscripts contained within this dissertation (Chapters 2 and 3). The first manuscript, which described the behavior and spectral properties of a relatively simple linear dye-DNA complex, achieved two milestones towards quantum information processing: (i) the identification of Cy5 dyes as promising candidates for the development of exciton-based devices and quantum gates due to the large Davydov splitting observed spectrally (i.e., a manifestation of dye-dye coupling and coherent exciton delocalization), and (ii) the data necessary to determine the physical parameters for a phenomenological theoretical model of exciton transport between Cy5 dyes within a DNA complex. The second manuscript, which encompassed a larger, more rigid, two-dimensional Holliday junction structure designed to form dye aggregates of a pre-determined size including dimers, trimers, and tetramers, validated the physical parameters used in the theoretical work for the first manuscript, showing that the same parameters can be used for other dye-DNA 
configurations. It also demonstrated that large Davydov splitting in dye aggregates can be achieved using a larger, more rigid two-dimensional Holliday junction structure. Taken together, the two manuscripts combined give confidence to the phenomenological theoretical model, which can be used as a predictive engineering tool for designing dyeDNA based excitonic devices and quantum gates, or as an analysis tool for determining dye configurations based on spectral data. 
TABLE OF CONTENTS

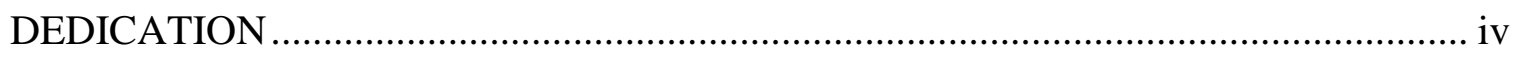

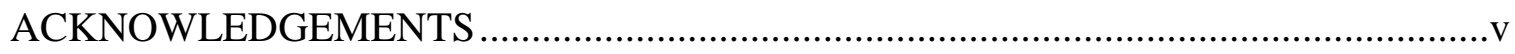

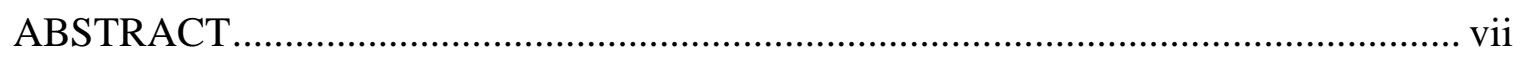

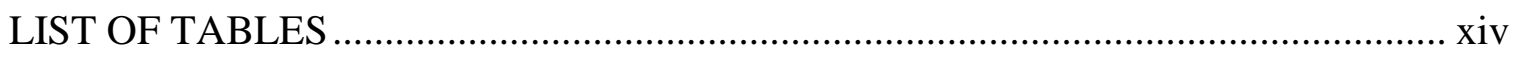

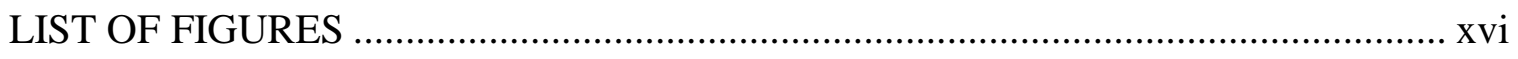

LIST OF ABBREVIATIONS/SYMBOLS ......................................................... xxiii

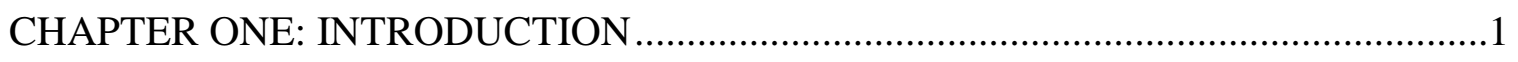

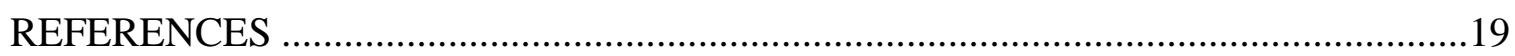

CHAPTER TWO: COHERENT EXCITON DELOCALIZATION IN A TWO-STATE DNA-TEMPLATED DYE AGGREGATE SYSTEM ..............................................26

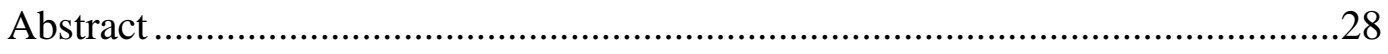

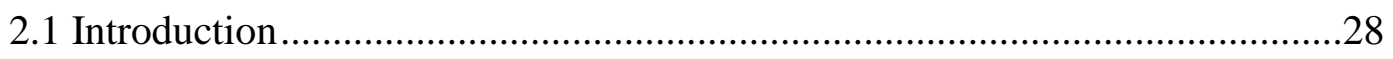

2.2 Theoretical Analysis of Optical Spectra for Structural Modeling ..................32

2.3 Kinetics and Thermodynamics of the Two-State Model ...............................33

2.3.1 Two-State Second-Order Kinetics Reaction Model .......................33

2.3.2 Fitting Reaction Kinetics Data ...................................................36

2.3.3 Effects of DNA Concentration on J- and H-Aggregate Populations 37

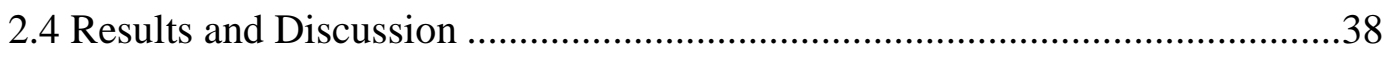

2.4.1 Optical Analysis of Dye-Aggregate States ...................................38 
2.4.2 Investigation of Aggregation State Reaction Kinetics and Thermodynamics

2.4.3 Effects of Salt and DNA Concentrations on J- and H-Aggregate

Populations.

2.4.4 Distance Dependence of Coherent Exciton Delocalization.

2.5 Conclusion

2.6 Acknowledgments .53

2.7 Supporting Information........................................................................ 54

2.7.1 Strand Sequences and Dye Details .54

2.7.2 Fitting of the Absorption and Circular Dichroism (CD) Data using the Kühn-Renger-May Model.....

2.7.3 Detailed Derivation of Second-Order Reactions Kinetics Model....69

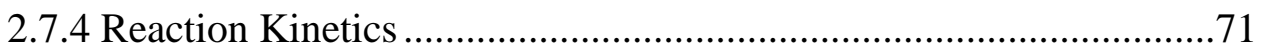

2.7.5 Spectral Analysis of Absorbance and Fluorescence Data.................78

2.7.6 Structural Characterization ................................................... 80

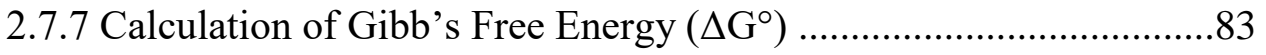

2.7.8 Salt and DNA Concentration Studies .........................................8

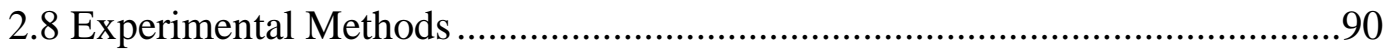

2.8.1 Preparation of DNA-Templated Dye Aggregate Constructs ............90

2.8.2 Optical Characterization of Dye Aggregates ...............................91

2.8.3 Structural Characterization of DNA-Templated Dye Aggregates ....93

REFERENCES

CHAPTER THREE: LARGE DAVYDOV SPLITTING AND STRONG

FLUORESCENCE SUPPRESSION: AN INVESTIGATION OF EXCITON

DELOCALIATION IN DNA-TEMPLATED HOLLIDAY JUNCTION DYE

AGGREGATES .104 


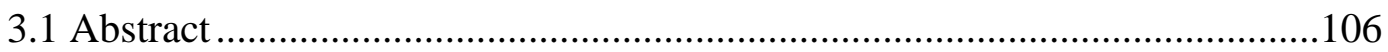

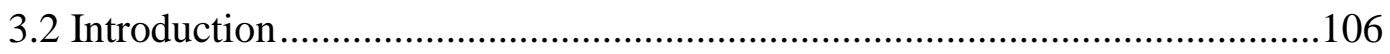

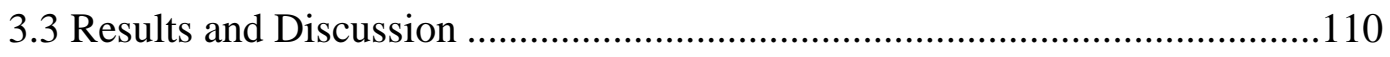

3.3.1 Optical Characterization of 4AJ Templated Dye Aggregates.........110

3.3.2 Theoretical Spectral Modeling of 4AJ Templated Dye

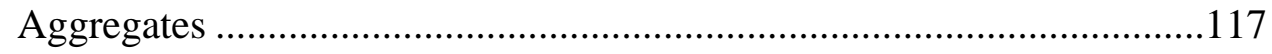

3.3.3 Optical Reporter Functionality ......................................................121

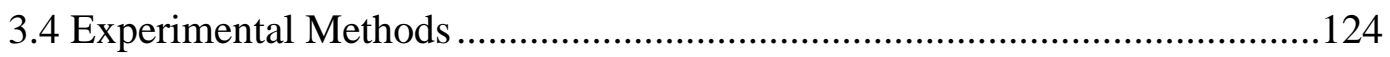

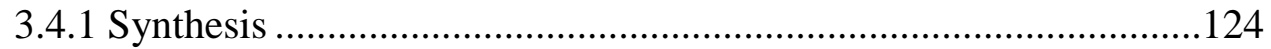

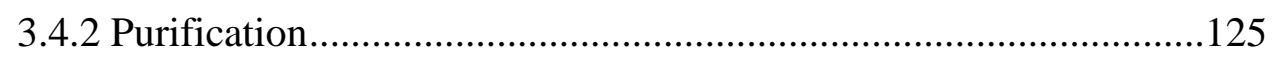

3.4.3 Optical Characterization ................................................................126

3.4.4 Optical Reporter Studies .............................................................126

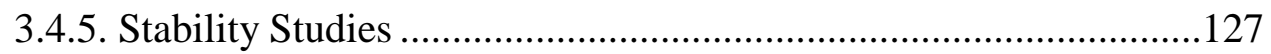

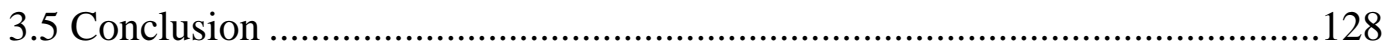

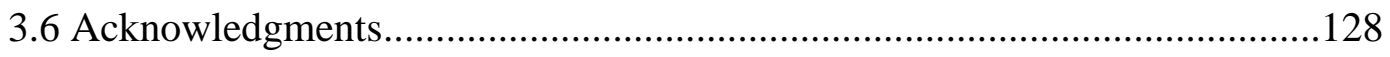

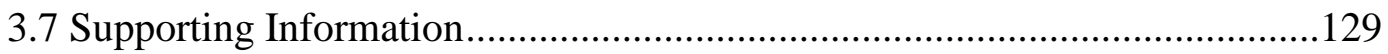

3.7.1 Strand Sequences and Dye Details ..................................................129

3.7.2 Spectral Analysis of Absorbance and Fluorescence Data................132

3.7.3 Fitting the Absorption and Circular Dichroism (CD) data using the Kühn-Renger-May Model.....................................................................135

3.7.4 Optical Reporter........................................................................139

3.7.5 Structural Characterization ............................................................141

3.7.6 Salt and DNA Concentration Studies ...........................................144

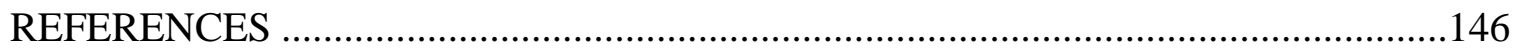


CHAPTER FOUR: SUMMARY AND FUTURE WORK 


\section{LIST OF TABLES}

Table 2.1. Activation energies $\left(E_{J \rightarrow H}\right.$ and $\left.E_{H \rightarrow J}\right)$, frequency factors $(A)$, and free energy $\left(\Delta G^{\circ}\right)$ values for aggregation state transitions. ............................. 45

Table 2.2 DNA Oligomer Sequences........................................................... 56

Table 2.3 List of Absorbance Peak Maxima, Extinction Coefficients, and Full Widths at Half Maximum (FWHM) for a variety of dye-DNA constructs.

Table 2.4 Kühn-Renger-May model fit parameters for the J-dimer and H-tetramer absorbance and CD spectra.

Table 2.5 Kühn-Renger-May model fitting outputs describing the dye orientations and positions.

Table 2.6 Kühn-Renger-May model fit parameters for the poor alternative fits of the H-tetramer absorbance and CD spectra shown in Figure 2.8.

Table 2.7 Kühn-Renger-May model fitting outputs describing the dye orientations and positions of the poor alternative fits for the H-tetramer absorbance and CD spectra shown in Figure 2.8.

Table 2.8 Reaction kinetics fitting parameters obtained for each data set shown in the top row of panels in Figure 2.10. All dye-DNA constructs were at 1.25 $\mu \mathrm{M}$ DNA concentration with $93.75 \mathrm{mM}$ added $\mathrm{MgCl}_{2}$. The number following the temperature describes the corresponding data set for which the fit was applied. 75

Table 2.9 Reaction kinetics fitting parameters obtained for each data set shown in the bottom row of panels in Figure 2.10. All dye-DNA constructs were at $1.25 \mu \mathrm{M}$ DNA concentration with $6.25 \mathrm{mM}$ added $\mathrm{MgCl}_{2}$. The number following the temperature describes the corresponding data set for which the fit was applied.

Table 2.10 Calculated forward $\left(k_{J \rightarrow H}\right)$ and reverse $\left(k_{H \rightarrow J}\right)$ reaction rate constants for the $\mathrm{J}_{0}-\mathrm{H}_{93.75}$ reactions shown in the upper row of panels in Figure 2.10. all dye-DNA constructs were at $1.25 \mu \mathrm{M}$ DNA concentration with $93.75 \mathrm{mM}$ 
added $\mathrm{MgCl}_{2} . K_{e q}$ values were found by fitting the absorbance data in Figure 2.17.

Table 2.11 Calculated forward $\left(k_{J \rightarrow H}\right)$ and reverse $\left(k_{H \rightarrow J}\right)$ reaction rate constants for the $\mathrm{H}_{100}-\mathrm{J}_{6.25}$ reactions shown in the bottom row panels in Figure 2.10. All dye-DNA constructs were at $1.25 \mu \mathrm{M}$ DNA concentration with $6.25 \mathrm{mM}$ added $\mathrm{MgCl}_{2} . K_{e q}$ values were found by fitting the absorbance data in Figure 2.17.

Table 2.12 Calculated forward $\left(k_{J \rightarrow H}\right)$ and reverse $\left(k_{H \rightarrow J}\right)$ reaction rate constants and corresponding equilibrium constants for $\mathrm{H}_{100}-\mathrm{J}_{6.25}$ obtained using the second method, see Eqs. (2.28) and (2.29). All dye-DNA constructs were at $1.25 \mu \mathrm{M}$ DNA concentrations with $6.25 \mathrm{mM}$ added $\mathrm{MgCl}_{2}$. $K_{\text {eq }}$ values were found by fitting the absorbance data in Figure 2.17 78

Table 2.13 Gaussian fitting parameters obtained for the peak maximum of each absorbance (Abs) and fluorescence (FL) data set shown in Figure 2.11. 80

Table 2.14 Equilibrium constant, $K_{e q}$, and fit regression coefficients, $R^{2}$. Samples were held at room temperature with $15 \mathrm{mM}$ added $\mathrm{MgCl}_{2}$. DNA concentrations were varied. 83

Table 3.1 Optical excitonic delocalization behavior per 4AJ construct .................. 117

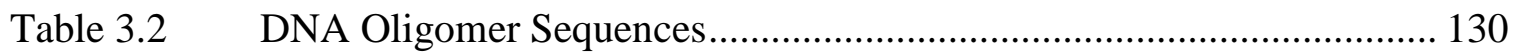

Table 3.3 Gaussian fitting parameters obtained for the peak maximum of each absorbance (Abs) and fluorescence (FL) data set shown in Figure 3.7. . 134

Table 3.4 Goodness of fit parameters for absorbance and CD spectra of various 4AJ configurations. 138

Table 3.5 Kühn-Renger-May model fitting outputs describing each dye orientation and position. 


\section{LIST OF FIGURES}

Figure 1.1. Illustration of typical dsDNA structure highlighting the various nanoscale features and modes of bonding that occur during hybridization. Adapted from Wikipedia. ${ }^{25}$........................................................................... 3

Figure 1.2. (a) Illustration of a DNA origami structure composed of a long scaffold strand (black) and corresponding short staple strands (color). (b) A variety of DNA origami shapes that can be designed (top two panels), constructed, and characterized using AFM (bottom two panels). The small white bars at the bottom of the images indicate $100 \mathrm{~nm}$ scale bars. Adopted from Rothemund. ${ }^{30}$

Figure 1.3. Illustration of the DNA brick concept. (a) Small staple strands act as Lego bricks such that (b) a library of bricks can be used to create a variety of 2D and 3D structures as shown in (c) and (d) depending upon the brick selection. The resulting DNA structure is similarly formed using a selection of strands to create the desired, finite shape..$^{38}$ 6

Figure 1.4. Diagram illustrating the spatial proximity of two dyes enabling FRET.... 7

Figure 1.5. Illustration of donor dye fluorescence overlap with acceptor dye absorbance.

Figure 1.6. Energy diagram illustrating the effects of dipole-dipole coupling between molecular dyes, resulting in splitting of the excited energy states. Allowed (solid black arrows) and forbidden (dashed black arrows) optical electronic state transitions are highly dependent on the relative geometrical orientation of the dyes, which can be categorized as (a) headto-tail (anit-parallel), (b) parallel, or (c) oblique stacking arrangements.. 15

Figure 1.7. Illustrations representing a variety of optical excitonic delocalization phenomenon. 16

Figure 2.1. Energy diagram illustrating the different excitation and relaxation pathways possible within (a) J-dimer, (b) H-dimer, and (c) oblique systems. Allowed absorption transitions are represented as solid black arrows, while dashed arrows indicate optically forbidden transitions. Blue and red arrows signify observed emission processes and nonradiative relaxation, respectively. The small arrows adjacent to each excited energy 
level represent the transition dipole moment of the corresponding molecules. Adapted from Kasha with permission. ${ }^{14}$

Figure 2.2. Schematic illustration of the constituents of the two-state second-order reaction kinetics model in which two J-dimers couple together to form an $\mathrm{H}$-tetramer through a four-way branch migration process. Also illustrated are the internal rearrangements within the core of the H-tetramer due to DNA breathing. As shown, dye dimers result when four-way branch migration steps cause the horizontal arms to become either longer or shorter than that of the dye tetramer, where all four dyes are located at the junction core

Figure 2.3. (a) Relative visible absorbance (black traces and ordinate axes), fluorescence emission (blue traces and ordinate axes), and (b) CD spectra of the monomer (15 $\mathrm{mM} \mathrm{MgCl} 2$ ). (c) Illustration depicting one possible representation of a single $\mathrm{Cy} 5$ dye internally functionalized onto a DNA duplex. (d) Relative visible absorbance, fluorescence emission, and (e) CD spectra for the J-dimer (duplex, $0 \mathrm{mM} \mathrm{MgCl} 2$ ). (f) Illustrative representation of the J-dimer stacked in a head-to-tail arrangement within a DNA duplex. (g) Relative visible absorbance, fluorescence emission, and (h) $\mathrm{CD}$ spectra of the H-tetramer (155 $\mathrm{mM} \mathrm{MgCl} 2)$ stacking arrangements. (i) Illustrative representation of the $\mathrm{H}$-tetramer formed within a four-armed DNA construct. Fluorescence spectra were acquired by exciting the dye constructs at their respective absorbance maxima. The FWHM of the absorbance (Abs) and fluorescence (FL) spectra were determined using a Gaussian fit, as described further in section 2.7.5. Theoretical fits (green dashed curves) were produced using in-house software as described further in section 2.7.2. All samples were prepared at $1.5 \mu \mathrm{M}$ DNA concentrations

Figure 2.4. Schematic illustrations of the reaction kinetics setup for solutions transitioning from (a) duplexes (J-dimers, $0 \mathrm{mM} \mathrm{MgCl} 2)$ to $4 \mathrm{AJs}(\mathrm{H}-$ tetramers, $93.75 \mathrm{mM} \mathrm{MgCl}_{2}$ ) and (b) 4AJs (H-tetramers, $100 \mathrm{mM} \mathrm{MgCl} 2$ ) to duplexes (J-dimers, $6.25 \mathrm{mM} \mathrm{MgCl}_{2}$ ). Initial dye-DNA solutions were prepared at DNA concentrations of $20 \mu \mathrm{M}$ to yield final concentrations of $1.25 \mu \mathrm{M}$ after dilution. Colors shown represent actual experimentally observed color changes. The depicted dye stacking arrangements are schematic representations and are not meant to imply specific geometric configurations. Corresponding reaction kinetics data of the time-dependent transitions: (c) $\mathrm{J}_{0} \rightarrow \mathrm{H}_{93.75}\left(0 \mathrm{mM} \rightarrow 93.75 \mathrm{mM} \mathrm{MgCl}_{2}\right.$ ) and (d) $\mathrm{H}_{100} \rightarrow$ $\mathrm{J}_{6.25}(100 \rightarrow 6.25 \mathrm{mM} \mathrm{MgCl} 2)$. Shown are representative data sets fit to Eq. (14) using the averaged rate constant measured over multiple runs for a given temperature. The log of the averaged forward $\left(k_{J \rightarrow H}\right)$ and reverse $\left(k_{H \rightarrow J}\right)$ rate constants obtained from a total of three independent measurements are plotted versus the inverse temperature for (e) $\mathbf{J}_{0} \rightarrow$ $\mathrm{H}_{93.75}$ and (f) $\mathrm{H}_{100} \rightarrow \mathrm{J}_{6.25}$, respectively, to determine the activation 
energies of the forward $\left(E_{J \rightarrow H}\right)$ and reverse $\left(E_{H \rightarrow J}\right)$ reactions based on the best fit to the data (black line). Error bars were determined using the standard deviation. Structural characterization of the aggregates using electrophoresis to monitor the migration of $(\mathrm{g}) \mathrm{J}$ - and $(\mathrm{h}) \mathrm{H}$-aggregates, prepared at 0 and $100 \mathrm{mM}$ added $\mathrm{MgCl}_{2}$, respectively, relative to a variety of DNA constructs (schematically represented). Absorbance spectra (i) of the excised and eluted bands were obtained for the J-dimer (red box) and the H-tetramer (blue box).

Figure 2.5. Observed UV-vis absorbance spectra with varied (a) salt (DNA concentration held constant at $1.5 \mu \mathrm{M}$ ) and (b) DNA (added $\mathrm{MgCl}_{2}$ concentration held constant at $15 \mathrm{mM}$ ) concentrations. Changes in J-dimer (black squares and ordinate axes) and $\mathrm{H}$-tetramer (blue circles and ordinate axes) fractions (based on their respective absorbance peaks) as a function of (c) salt (i.e., $\mathrm{MgCl}_{2}$ ) and (d) DNA concentrations. The dashed lines connecting the points in panel c are for visual aid only, while the curves in panel d were obtained by fitting the data according to Eqs (19) and (20). Each spectrum was acquired at room temperature.

Figure 2.6 UV-Vis absorbance spectra documenting changes in the optical properties of dye aggregates as the number of bps separating the dye pair is increased. Samples were prepared at $1.0 \mu \mathrm{M}$ DNA concentration with 15 $\mathrm{mM} \mathrm{MgCl} 2$ added. Spectra are normalized by DNA concentration.

Figure 2.7. Molecular structure of the Cy5 dye adapted from IDT. The two long carbon tethers are used to attach onto the DNA oligomers. The attachment sites by which the dye is covalently bound to the DNA backbone are indicated by 5' and 3', representing the directionality of the attachment scheme.

Figure 2.8. Three poor alternative fits of the H-tetramer absorbance (top panels) and CD (middle panels) spectra using the Kühn-Renger-May model to quantitatively compare the goodness of fit for a variety of dye configurations (bottom panels), including (a) square, (b) in line, and (c) stacked configurations.

Figure 2.9. Raw (i.e., non-normalized) reaction kinetics data demonstrating aggregate state transitions at $20^{\circ} \mathrm{C}, 22{ }^{\circ} \mathrm{C}, 25^{\circ} \mathrm{C}$, and $30{ }^{\circ} \mathrm{C}$. The dye-DNA constructs $(1.25 \mu \mathrm{M}$ DNA concentration) were monitored at $562 \mathrm{~nm}$, the $\mathrm{H}$-tetramer peak absorption maximum, in order to eliminate interference from the monomer. The first $\sim 10-12$ minutes of the data examines any baseline absorption due to the TAE buffer, after which the dye-DNA constructs were added into the solution. The top row of panels $\left(\mathrm{J}_{0}-\mathrm{H}_{93.75}\right)$ show switching from the $\mathrm{J}$-dimer state initially at $0 \mathrm{mM} \mathrm{MgCl}_{2}$ to predominantly $\mathrm{H}$-tetramer states at a final salt concentration of $93.75 \mathrm{mM}$ $\mathrm{MgCl}_{2}$. The bottom row of panels $\left(\mathrm{H}_{100}-\mathrm{J}_{6.25}\right)$ demonstrate switching from 
$\mathrm{H}$-tetramers initially at $100 \mathrm{mM} \mathrm{MgCl} 2$ to mainly J-dimers at a final salt concentration of $6.25 \mathrm{mM} \mathrm{MgCl} 2$. Small spikes evident in the data at the time of injection are the result of momentarily inserting a pipette into the incident light beam path

Figure 2.10. Reaction kinetics data demonstrating J-dimer to H-tetramer (top row of panels) and H-tetramer to J-dimer (bottom row of panels) transitions. The black curves shown in each plot represent the fit used to calculate the second-order reaction rate constants. Fitting parameters and regression coefficient parameters are listed in Tables 2.8 and 2.9. Reaction rate constants are calculated from the fits are listed in Table 2.12

Figure 2.11. Relative UV-Vis absorbance spectra (top panels) and fluorescence (bottom panels) of the monomer (15 $\mathrm{mM}$ added $\left.\mathrm{MgCl}_{2}\right)$, J-dimer ( $0 \mathrm{mM}$ added $\mathrm{MgCl}_{2}$ ), and $\mathrm{H}$-tetramer (155 $\mathrm{mM}$ added $\mathrm{MgCl}_{2}$ ) from left to right, respectively. Each dataset was fit to a series of Gaussian curves to obtain information regarding the peak positions and full width at half maximums (FWHMs). All solutions of dye-DNA constructs were prepared at $1.5 \mu \mathrm{M}$.

Figure 2.12. Illustrations of completed (a) three, (b) four, and (c) six-armed structures that were used as controls for monitoring the migration of duplexed strands during gel electrophoresis. Strand sequences are listed in Table 2.2

Figure 2.13. UV-Vis absorbance spectra normalized by DNA concentration showing spectral changes as a function of salt $(\mathrm{NaCl})$ concentration. Note that the scans were not necessarily taken at chemical equilibrium, but still indicate that a two-state quantum coherent system exists. The absorbance spectrum acquired by Markova et al. has been inserted into the data set at the appropriate salt concentration to demonstrate reproducibility. 83

Figure 2.14. Raw UV-Vis absorbance spectra taken after completed reaction kinetics runs for both $\mathrm{J}_{0}-\mathrm{H}_{93.75}$ reactions (left side panel) and $\mathrm{H}_{100}-\mathrm{J}_{6.25}$ reactions (right side panel). All solutions of dye-DNA constructs consisted of 1.25 $\mu \mathrm{M}$ DNA concentrations. Panels on the left were taken at $93.75 \mathrm{mM}$ $\mathrm{MgCl}_{2}$ concentrations, while the panels on the right were taken at 6.25 $\mathrm{mM} \mathrm{MgCl} 2$ concentration.

Figure 2.15. UV-Vis absorbance spectra (normalized by area) taken after completed reaction kinetics runs for both $\mathrm{J}_{0} \rightarrow \mathrm{H}_{93.75}$ reactions (left side panel) and $\mathrm{H}_{100} \rightarrow \mathrm{J}_{6.25}$ reactions (right side panel). Each scan was fit to Eq. (2.21) to give relative percentages of each individual aggregate state: $\boldsymbol{m}$ (monomer), $\boldsymbol{j}$ (J-dimer), and $\boldsymbol{h}$ (H-tetramer). All solutions of dye-DNA constructs consisted of $1.25 \mu \mathrm{M}$ DNA concentrations. Panels on the left 
were taken at $93.75 \mathrm{mM} \mathrm{MgCl} 2$ concentrations, while the panels on the right were taken at $6.25 \mathrm{mM} \mathrm{MgCl} 2$ concentration.

Figure 2.16. UV-Vis absorbance spectra of PAGE purified monomer $(\boldsymbol{M}(\boldsymbol{\lambda})$; green curve), J-dimer $(\boldsymbol{J}(\boldsymbol{\lambda})$; red curve), and H-tetramer $(\boldsymbol{H}(\boldsymbol{\lambda})$; blue curve). all spectra have been normalized by area and subsequently used to determine the percentages of each state present in non-purified samples. 87

Figure 2.17. UV-Vis absorbance scans (normalized by area) showing spectral changes as a function of salt ( $\mathrm{MgCl}_{2}$, left panels) and DNA (right panels) concentrations. Each scan is fit to Eq. (2.75) to give relative percentages of each individual aggregate state: $\boldsymbol{m}$ (monomer), $\boldsymbol{j}$ (J-dimer), and $\boldsymbol{h}(\mathrm{H}-$ tetramer).

Figure 3.1. Schematics and energy diagrams for various dye aggregate arrangements: (a) monomer, (b) H-dimer, (c) J-dimer, and (d) oblique dimer. The top schematic shows dye molecules represented as purple spheres, while the dipole orientation is depicted by black arrows. Each energy diagram shows a ground energy state $\left(\mathrm{E}_{0}\right)$, the first excited energy state $(\mathrm{E})$ for the monomer, and the excited energy state's split energy levels (higher energy, $\mathrm{E}^{+}$and lower energy, $\mathrm{E}^{-}$) for dimers due to exciton delocalization. Allowed energy transitions are provided as solid black arrows and forbidden transitions are shown as dashed black arrows. Adapted from Kasha with permission. ${ }^{4}$..... 108

Figure 3.2. Absorbance (black traces in left column), fluorescence (blue traces), and circular dichroism (black traces in right column) spectra corresponding to the 4AJ templated $(\mathrm{a}, \mathrm{b})$ monomer, $(\mathrm{c}, \mathrm{d})$ adjacent dimer, $(\mathrm{e}, \mathrm{f})$ transverse dimer, $(\mathrm{g}, \mathrm{h})$ trimer, and $(\mathrm{i}, \mathrm{j})$ tetramer dye aggregates. The inset in (i) shows the suppressed fluorescence emission peak at $664 \mathrm{~nm}$ that occurs due to a forbidden energy transfer mechanism. All spectra have been normalized by DNA concentration. The fluorescence studies were performed by monitoring the emission over a range of wavelengths and exciting the aggregates at their respective absorbance maxima. All samples were prepared at $10 \mu \mathrm{M}$ DNA concentration in a $1 \times$ TAE buffer solution with $15 \mathrm{mM} \mathrm{MgCl} 2$ added, PAGE purified, and normalized by the resulting DNA concentrations $(\sim 0.5-2.5 \mu \mathrm{M})$. 114

Figure 3.3. Absorbance (a,d,g, and j) and CD (b, e, h, and k) spectral fits with corresponding structures (c, f, i, and $\mathrm{l}$ ) of the adjacent dimer, transverse dimer, trimer, and tetramer aggregates, respectively. Experimental data sets are shown as black open circles, while KRM theoretical fits are given as red curves. All samples were prepared at $10 \mu \mathrm{M}$ DNA concentration in a $1 \times$ TAE buffer solution with $15 \mathrm{mM} \mathrm{MgCl} 2$ added, PAGE purified, and normalized by the resulting DNA concentrations $(\sim 0.5-2.5 \mu \mathrm{M})$. 
Fluorescence measurements were obtained by exciting samples at their respective absorption maxima.............................................................. 120

Figure 3.4. (a) Schematic illustration and (b) experimental demonstration of the 4AJ tetramer undergoing strand-invasion using fluorescence detection $(\lambda \mathrm{fl}=$ $666 \mathrm{~nm}$ ). (c) Schematic illustration and (d) experimental demonstration of the $4 \mathrm{AJ}$ tetramer formation via DNA hybridization using UV-Vis absorbance, or colorimetric, detection $(\lambda \mathrm{abs}=565 \mathrm{~nm})$

Figure 3.5. Molecular structure of the Cy5 dye adapted from IDT. The two long carbon tethers represent the directionality of the attachment scheme onto the phosphate backbone of the DNA.

Figure 3.6. Illustration depicting the complementary DNA domains of each strand (Cy5-A, Cy5-B, Cy5-C, Cy5-D, etc.) that hybridize to form the (a) complete four-armed junction (4AJ) structure, (b) absorbance detection scheme, and (c) fluorescence detection scheme. The locations of Cy5 molecules are indicated by purple spheres; however, do not suggest orientation.

Figure 3.7. Peak fitting performed on both absorbance (left panels) and fluorescence (right panels) spectra (black circles) using a Gaussian curve (solid curves) and a least squares fitting procedure for the 4AJ (a) monomer, (b) adjacent dimer, (c) transverse dimer, (d) trimer, and (e) tetramer configurations. The fluorescence studies were performed by monitoring the emission over a range of wavelengths and exciting the aggregates at their respective absorbance maxima

Figure 3.8. Optical reporter data demonstrating transitions between non-fluorescent and fluorescent states for the fluorescence-based detection scheme (a) and between non-absorbing and absorbing states for the absorbance-based detection scheme (b). In the fluorescence-based detection scheme, one of the $4 \mathrm{AJ}$ strands was augmented with a $10 \mathrm{nt}$ toehold to enable toeholdmediated strand invasion in order to remove of one of the dye-labeled strands. Upon removal of one of the dyes from the tetramer aggregate, excitonic quantum coherence between aggregates ceases and the dyes fluoresce. Samples were excited at $565 \mathrm{~nm}$ (the tetramer absorbance maximum) and monitored at $666 \mathrm{~nm}$ (the peak emission). The experiments were performed at room temperature with a DNA concentration of $\sim 0.4$ $\mu \mathrm{M}$. In the absorbance-based detection scheme, absorbance changes were induced by the combination of two halves of the 4AJ via DNA hybridization, thereby enabling tetramer formation and promoting absorbance at $565 \mathrm{~nm}$. The fluorescence-based experiments were performed at room temperature with a DNA concentration of $\sim 0.1 \mu \mathrm{M}$. The first 10 minutes of the data, which examines any baseline 
fluorescence/absorption due to the initial construct, has been removed along with small spikes that resulted from momentarily inserting the pipette into the incident light beam path.

Figure 3.9. Absorbance (bottom panels) and fluorescence (top panels) spectra depicting the optical behavior of the 4AJ aggregate structures measured pre- (left panels, $t=0$ minutes) and post (right panels, $t=30$ minutes) reaction kinetic run as demonstrated in Figure 3.8a.

Figure 3.10. (a) Images of PAGE gel containing various 4AJ-templated dye-DNA constructs. All lanes that consist of DNA oligomers labeled with a single Cy5 dye molecule are identified by the violet box. Complexes that include two strands that are half-complementary and form a dye dimer are signified by the blue box. Orange boxes indicates well-formed 4AJ constructs with a variety of dye arrangements. The yellow box is used to highlight higher order DNA (most likely octamer) structures that form spontaneously in solution. Note that Lane 10, corresponding to the tetramer, does not have a band in the yellow-boxed region as compared to Lanes 7-9, indicating that higher ordered structures are not prevalent. Color-coded arrows direct towards the corresponding illustration of the DNA construct. To verify that the tetramer complexes were stable and did not form "octamer: structures while in equilibrium, the yellow and orange bands of (b) Lane 9 and (c) Lane 10 were excised, allowed to elute into solution and reach equilibrium over 24 hours, and then re-purified in a PAGE gel.

Figure 3.11. UV-Vis absorbance spectra demonstrating the stability of the 4AJ tetramer in solution with variable salt (i.e., $\mathrm{MgCl}_{2}$, left panel) and DNA (right panel) concentrations. The salt stability experiment was conducted with samples held at constant DNA concentrations of $1.5 \mu \mathrm{M}$, while DNA stability samples were conducted with samples held at constant salt concentrations of $15 \mathrm{mM} \mathrm{MgCl}$

Figure 4.1. Illustration of an excitonic beam splitter with optical readouts. Adapted by Yurke and Kuang. ${ }^{82}$ 157 


\section{LIST OF ABBREVIATIONS/SYMBOLS}

\begin{tabular}{ll} 
a.u. & Arbitrary Units \\
$\mathrm{bp}$ & Base-pair \\
$\mathrm{cm}$ & Centimeter \\
$\mathrm{CD}$ & Circular Dichroism \\
$\mathrm{Cy} 5$ & Cyanine dye \\
${ }^{\circ} \mathrm{C}$ & Degrees Celsius \\
$\mathrm{DNA}$ & Deoxyribonucleic acid \\
$\mathrm{dsDNA}$ & Double stranded DNA \\
$\mathrm{eV}$ & Electron volt \\
$K_{e q}$ & Equilibrium rate constant \\
EC-CD & Excitonically-Coupled Circular Dichroism \\
FMO & Fenna-Matthews Olson complex \\
$R_{0}$ & Förster radius \\
FRET & Förster Resonance Energy Transfer \\
$4 \mathrm{AJ}$ & Four-Armed Junction \\
FWHM & Full-Width at Half-Maximum \\
$\Delta \mathrm{G}^{\circ}$ & Gibb's Free Energy \\
$\mathrm{HPLC}$ & Kigh-performance liquid chromatography \\
$\mathrm{ISGC}$ & Kilo Joule \\
\hline &
\end{tabular}




\begin{tabular}{|c|c|}
\hline $\mathrm{Mg}^{2+}$ & Magnesium Cation \\
\hline $\mathrm{MgCl}_{2}$ & Magnesium Chloride \\
\hline MSE & Materials Science and Engineering Department \\
\hline$\mu \mathrm{L}$ & Microliter \\
\hline$\mu \mathrm{M}$ & Micromolar \\
\hline $\mathrm{meV}$ & Milli-electron volt \\
\hline $\mathrm{mK}$ & Milli-Kelvin \\
\hline $\mathrm{mM}$ & Millimolar \\
\hline Milli-Q & Millipore trademark name \\
\hline $\mathrm{mV}$ & Millivolts \\
\hline $\min$ & Minutes \\
\hline M & Molar \\
\hline $\mathrm{nm}$ & Nanometer \\
\hline NASA & National Aeronautics and Space Administration \\
\hline NSF & National Science Foundation \\
\hline NIR & Near-Infrared \\
\hline nt & Nucleotide \\
\hline PAGE & Poly Acrylamide Gel-Electrophoresis \\
\hline Qbit & Quantum Bit \\
\hline$Q_{D}$ & Quantum Efficiency \\
\hline$k$ & Reaction rate constant \\
\hline$n$ & Refractive index \\
\hline $\mathrm{rcf}$ & Relative Centrifugal Force \\
\hline
\end{tabular}




\begin{tabular}{|c|c|}
\hline $\mathrm{Na}^{+}$ & Sodium Cation \\
\hline $\mathrm{NaCl}$ & Sodium Chloride \\
\hline$s$ & Seconds \\
\hline SOG & Special Optical Glass \\
\hline$T$ & Temperature \\
\hline $3 \mathrm{D}$ & Three-dimensional \\
\hline$t$ & Time \\
\hline$\mu$ & Transition dipole moment \\
\hline TAE & Tris(hydroxymethyl)aminomethane, acetic acid, and \\
\hline & ethylenediaminetetraacetic acid \\
\hline $2 \mathrm{D}$ & Two-dimensional \\
\hline UV & Ultraviolet \\
\hline Vis & Visible \\
\hline V & Volts \\
\hline$\lambda$ & Wavelength \\
\hline
\end{tabular}




\section{CHAPTER ONE: INTRODUCTION}

Until recently, the idea of quantum computing seemed more like science fiction than imminent reality ever since its first conception in the early 1980's. ${ }^{1,2}$ In 1999, the Canadian company D-Wave introduced the first 'quantum computer'. Though the company faced harsh criticism owing to their insufficient evidence of quantum speedup, ${ }^{3,}$

${ }^{4}$ their quantum computer is now being utilized by world-class organizations including Lockheed Martin, Google, and NASA. ${ }^{5}$ The superconductor-based design of D-Wave's quantum computer necessitates special laboratory facilities to maintain near-absolute zero temperatures $(15 \mathrm{mK})$ to maintain coherence of the quantum bits (Qbits), preventing the system from being monetarily feasible for large scale commercialization. These nearabsolute zero temperatures are imperative for reducing the noise in the system and consequently increasing the accuracy of the computations. Due to this significant constraint, the development of an alternative quantum computational system that can operate at room temperature is extremely attractive.

Looking to biology for inspiration, the recent observation of exciton delocalization at ambient temperatures within the protein-based Fenna-Matthews-Olson (FMO) complex found in naturally occurring photosynthetic systems suggests a promising path towards inexpensive organic room temperature quantum computation. ${ }^{6-10}$ Photosynthetic systems such as the FMO complex are composed of dye aggregates arranged with nanometer precision by a protein scaffold. ${ }^{11}$ The extremely small distances separating the dyes results in dipole-dipole coupling between the molecules, enabling 
excitonic delocalization across all of the dyes to create a superposition of the exciton (i.e., electron-hole pair). This quantum mechanical effect causes the exciton to efficiently and effectively transfer from the antennae of the photosynthetic complex to its reaction center via quantum coherent wave-like behavior, as opposed to an inefficient classical particlelike hopping mechanism. ${ }^{7,12-18}$

Though nature has successfully optimized the folding of proteins into architectures that enhance rather than impede exciton delocalization, anthropogenically designing or predicting a protein-based scaffold is difficult a priori owing to the complex nature of amino-acid interactions. An alternative biomaterial, deoxyribose nucleic acid (DNA), can be used instead to more readily enable precise control over the placement of dyes. Structural DNA nanotechnology was pioneered by Nadrian Seeman in 1982 with the demonstration of DNA as a self-assembling material for designing and fabricating complex two- (2D) and three-dimensional (3D) crystalline structures. ${ }^{19-24}$ DNA's use as a structural platform takes advantage of the simplicity and elegance of the biological polymer's design rules. A single strand of DNA (ssDNA) is composed of three major constituents: (a) a phosphate backbone, (b) a pentose sugar (ribose), and a nitrogenous base [i.e., adenine (A), thymine $(T)$, cytosine $(C)$, or guanine $(\mathrm{G})$, Figure 1.1]. The nitrogenous bases, or nucleotides, are distinguished into two groups: pyrimidines ( $\mathrm{T}$ and $\mathrm{C}$, which have one carbon-nitrogen ring) and purines (A and G, which have two carbonnitrogen rings). The nucleotides form chains or single strands of DNA by covalent bonding between the sugar of one nucleotide and the phosphate backbone of another adjacent molecule, giving rise to a well-defined spacing between each nucleotide of $\sim 0.36 \mathrm{~nm}$. Double stranded DNA (dsDNA), forms a right-handed double helix composed 
of two single strands of DNA in which complementary bases (i.e., A-T and C-G)

hybridize together, stabilized by hydrogen bonding and base-stacking (i.e., pi-pi overlap,

Figure 1.1). Additionally, due to the asymmetry of the two ends of the phosphate

backbone (i.e., 5' end of the sugar versus the 3' end of the sugar) the two strands of the

double helix run antiparallel to each other, thereby providing structural directionality.

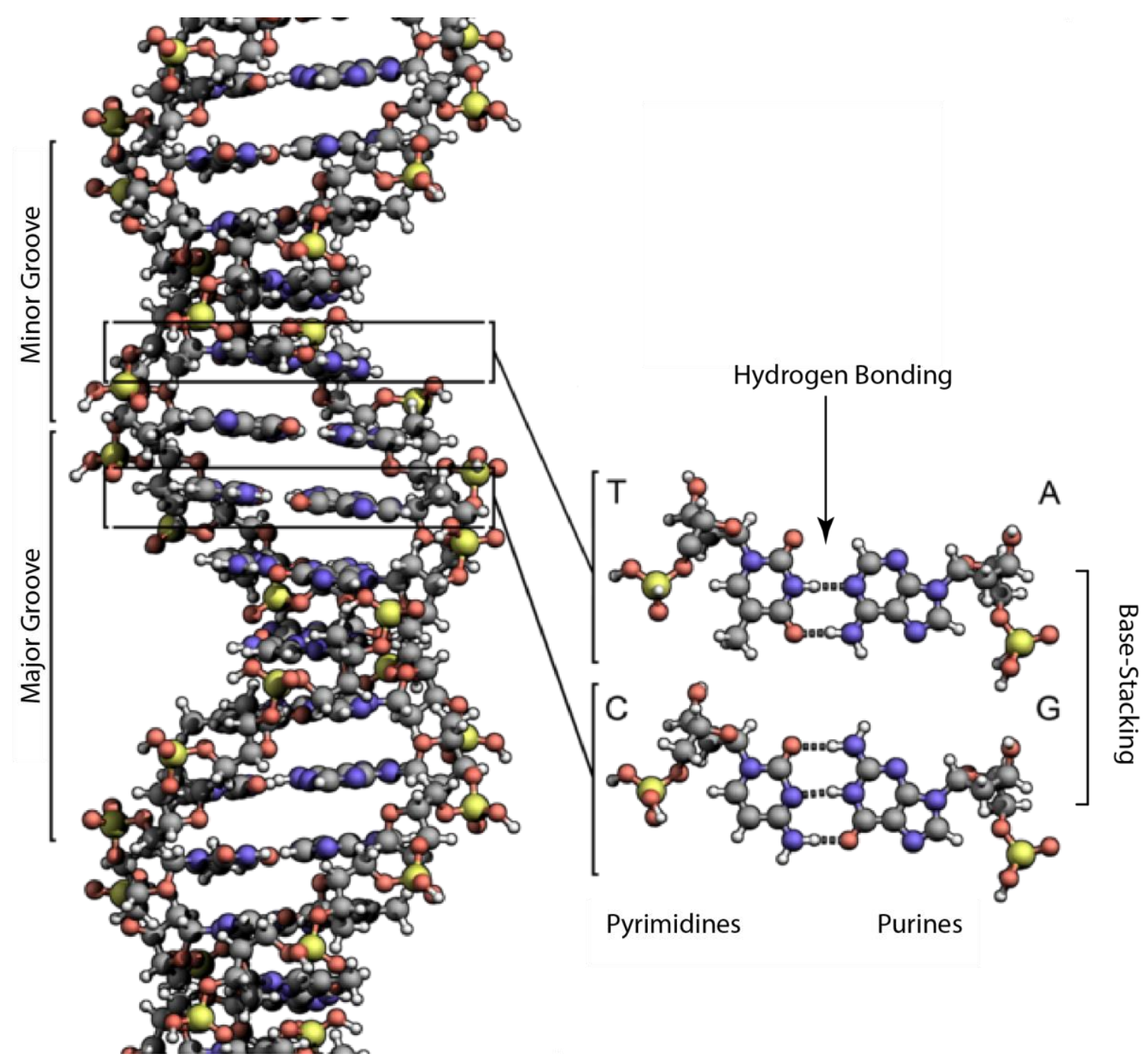

Figure 1.1. Illustration of typical dsDNA structure highlighting the various nanoscale features and modes of bonding that occur during hybridization. Adapted from Wikipedia. ${ }^{25}$ 
Capitalizing on the simple design rules offered by DNA, Paul Rothemund introduced the technique of DNA origami in 2006, which revolutionized the field of DNA nanotechnology, as has been widely acknowledged within the scientific community. ${ }^{26-29}$ DNA origami involves the folding of a long ssDNA scaffold strand (black strand, Figure 1.2a), derived from circular genomic DNA, into a finite, nanoscale structure using short staple strands (colored strands, Figure 1.2a). Similar to the construction of large arrays, as introduced by Seeman, DNA origami is a bottom-up approach in which trillions of structures are constructed at once using a one-pot selfassembly technique that is driven thermodynamically and kinetically towards the desired shape.

a

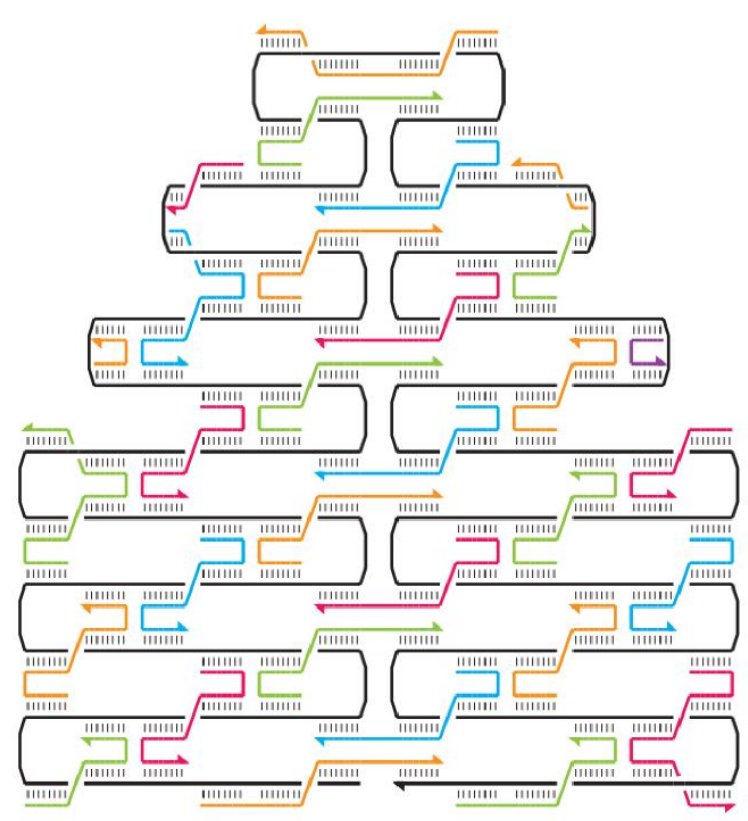

b
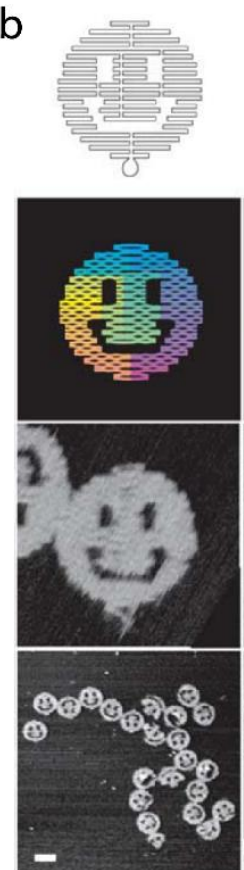
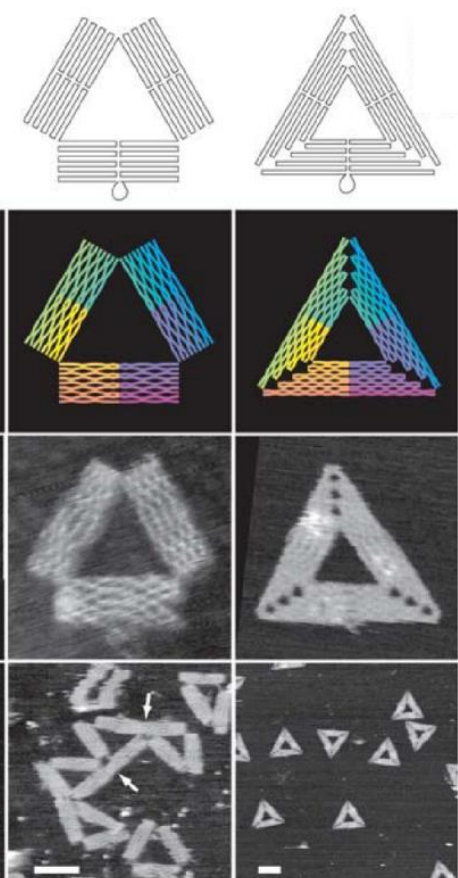

Figure 1.2. (a) Illustration of a DNA origami structure composed of a long scaffold strand (black) and corresponding short staple strands (color). (b) A variety of DNA origami shapes that can be designed (top two panels), constructed, and characterized using AFM (bottom two panels). The small white bars at the bottom of the images indicate $100 \mathrm{~nm}$ scale bars. Adopted from Rothemund. ${ }^{30}$ 
Design and modeling of DNA origami shapes and structures, as shown in the first and second panel of Figure 1.2b, can be achieved using free software, such as caDNAno, ${ }^{31-33}$ NUPACK, ${ }^{34-36}$ and $\mathrm{CanDo}^{37}$ to rapidly prototype and model the assembled DNA structure as well as its mechanical, thermodynamical, and structural properties. The most common technique used to characterize DNA origami structure formation is atomic force microscopy (AFM). AFM images of DNA origami structures are depicted in the bottom two panels of Figure 1.2b. AFM is a scanning probe microscopy technique that enables non or minimally destructive near atomic resolution topographical mapping of soft materials such as DNA in their native aqueous environment.

Subsequent to the development of DNA origami, a method for fabricating twoand three-dimensional DNA bricks was announced in which discrete and complicated DNA nanostructures could be modularly assembled using only short staple strands (Figure 1.3). ${ }^{38,39}$ Each staple strand consists of four unique sequence domains that selectively hybridize to four independent, neighboring strands, and, like Legos, can be joined together to create larger structures (Figure 1.3a). Thus, a multitude of molecularly precise structures and architectures can be fabricated from a master library of DNA strands by selectively choosing the strands necessary for each design (Figure 1.3b-e). 

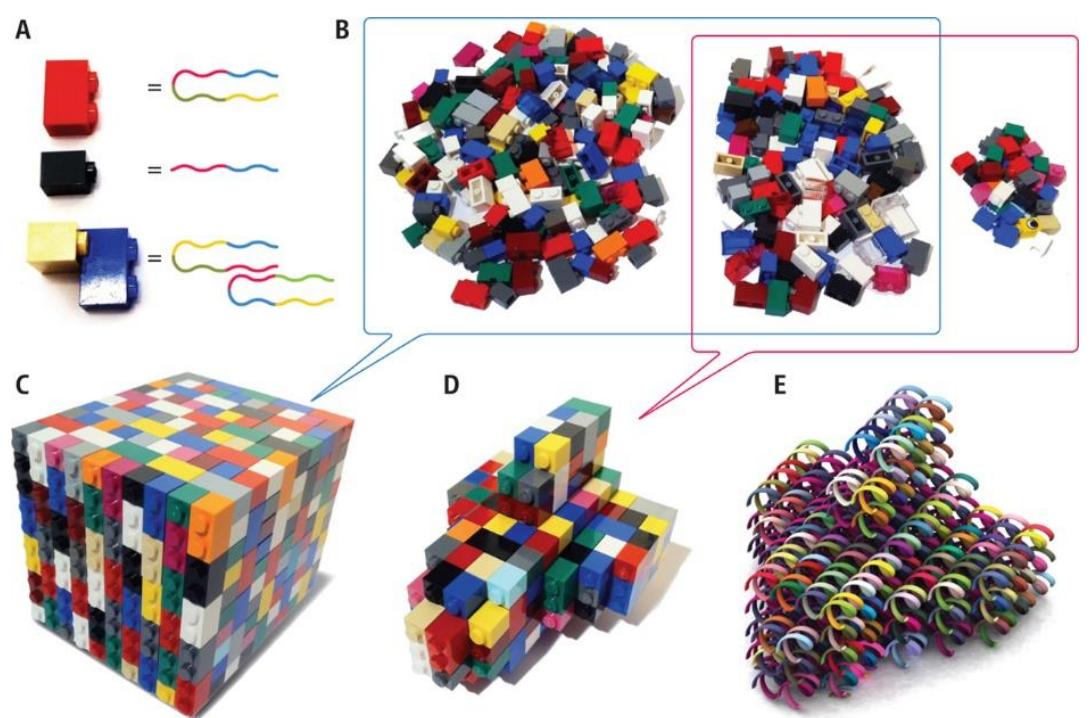

Figure 1.3. Illustration of the DNA brick concept. (a) Small staple strands act as Lego bricks such that (b) a library of bricks can be used to create a variety of $2 D$ and 3D structures as shown in (c) and (d) depending upon the brick selection. The resulting DNA structure is similarly formed using a selection of strands to create the desired, finite shape. ${ }^{38}$

Both DNA origami and DNA bricks offer programmability by which optically active nanoparticles (e.g., metal nanoparticles, organic dyes, or inorganic quantum dots) can be precisely positioned and arranged onto the DNA nanostructures. Importantly, the physical proximity with which nanoparticles can be arranged onto the DNA nanostructures results in near-field interactions, including quantum coherence and excitonic energy transfer. In the case of organic molecular dyes, excitonic energy transfer most commonly culminates as incoherent Förster resonance energy transfer (FRET) or coherent exciton delocalization, depending on the strength of dipole-dipole coupling between the molecules. Both of these phenomena are described in further detail below.

Förster resonance energy transfer (FRET) is an exquisitely sensitive distancedependent phenomenon that has been utilized to probe a wide range of biological, biophysical, and optoelectronic interactions. FRET is a physical process whereby energy in the form of excitons (i.e., boson-like quasi-particles comprised of electron-hole pairs) 
is transferred incoherently and nonradiatively from a donor dye to an acceptor dye through intermolecular dipole-dipole coupling. ${ }^{40}$ In order for FRET to occur, four criteria must be met: (i) the two dyes must be physically close to one another ( $\sim-12 \mathrm{~nm}$, Figure 1.4), (ii) the fluorescence emission spectrum of the donor dye must overlap with the absorbance spectrum of the acceptor dye (Figure 1.4), (iii) the transition dipole moments of the donor and acceptor must lie approximately parallel to one another, and (iv) the fluorescence (radiative) lifetime of the donor molecule must be sufficiently long relative to the FRET rate. The efficiency of the FRET process $\left(E_{F R E T}\right)$ has been shown to be highly dependent on the radial distance $(r)$ between the donor and acceptor dye, as given by: 41

$$
E_{F R E T}=\frac{1}{1+\left(\frac{r}{R_{0}}\right)^{6}}
$$

where $R_{0}$ is the Förster radius, defined as the dye-dye separation distance at which half of the donor excitation energy is transferred to the acceptor dye, such that the FRET efficiency is $50 \%$.

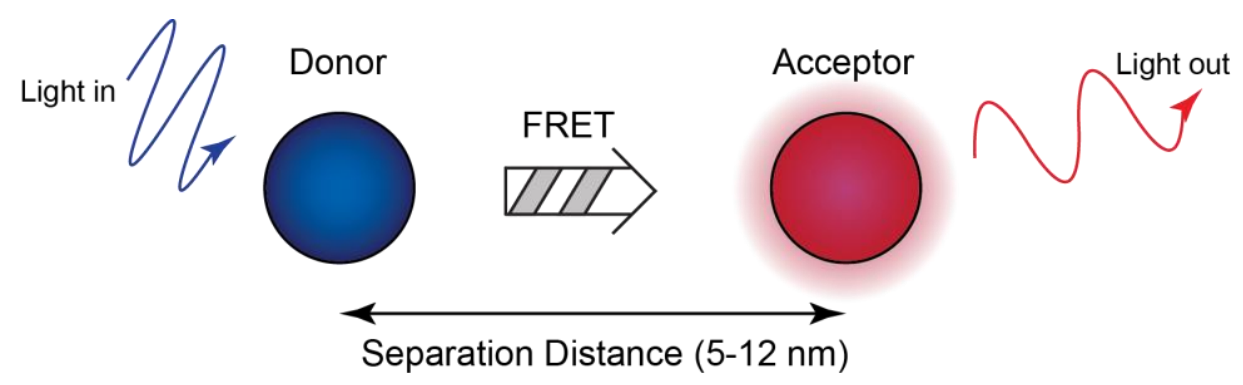

Figure 1.4. Diagram illustrating the spatial proximity of two dyes enabling FRET. 
The Förster radius, in angstroms $(\AA)$, can be expressed as a function of the spectral overlap integral $(J)$, quantum efficiency $\left(Q_{D}\right)$ of FRET transfer, index of refraction $(n)$, and relative dye orientation $\left(\kappa^{2}\right):^{42}$

$$
R_{0}^{6}(\AA)=8.79 \times 10^{-5} Q_{D} n^{-4} \kappa^{2} J
$$

where the spectral overlap integral is written as a function of integrals: ${ }^{42}$

$$
J\left(M^{-1} c m^{-1} n m^{4}\right)=\frac{\int \epsilon(\lambda) f(\lambda) \lambda^{4} d \lambda}{\int f(\lambda) d \lambda} .
$$

The extinction coefficient of the acceptor dye absorbance cross-section is given as $\epsilon(\lambda)$, the emission spectrum of the donor dye is $f(\lambda)$, with both given as a function of wavelength $(\lambda)$. When the dyes have a fixed orientation relative to each other one has: ${ }^{43}$, 44

$$
\kappa=\cos \left(\theta_{T}\right)-3 \cos \left(\theta_{1}\right) \cos \left(\theta_{2}\right)
$$

where $\theta_{T}$ is the total angle between the transition dipole moments of the two neighboring dyes and $\theta_{1}$ and $\theta_{2}$ are the individual angles of the transition dipole moments for dye 1 and 2, respectively, relative to the line running between the centers of the two dyes. For solution based systems, $\kappa^{2}$, derived from Eq. (1.4), is typically taken to be $2 / 3$ based on the expected random (isotropic) orientation of dyes attached to a rotationally free linker. 


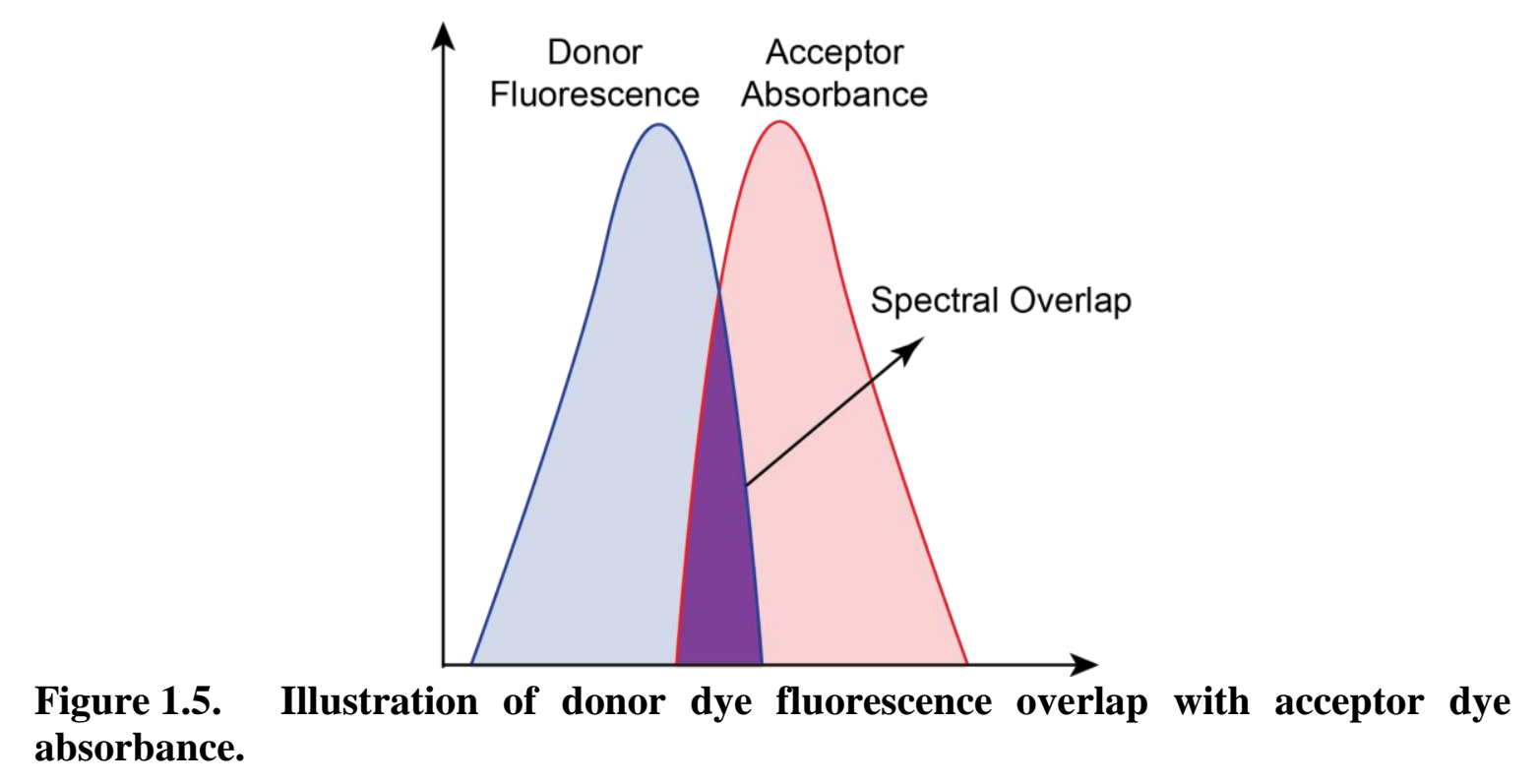

Due to its exquisite distance sensitivity, FRET-based systems have often been employed as molecular rulers. ${ }^{44,45}$ Additionally, FRET systems have been instrumental in the imaging of biological phenomena (both in vivo and in vitro), and are considered one of the leading technologies for excitonic-based devices. The potential advantages of FRET-based systems result from three main properties: (1) nanosecond excitonic energy transfer speeds, (2) all optical inputs and outputs, and (3) the nanometer scale distancedependence ( 5-12 nm) of FRET efficiency which allows for the development of sensitive biosensors. The two main disadvantages of FRET-based systems are the inevitable loss of energy as it is transferred between dyes and the stepwise relegation of excited states at higher energies to lower energies. Additionally, FRET is inherently 'incoherent' due to the nearly complete relaxation of the exciton due to environmental interactions prior to transferring its energy to the neighboring dye. These limitations can be partially overcome by selecting dye pairs with the greatest spectral overlap and/or by optimizing the distance between the dyes. However, upon further decreasing the separation between dyes below the Förster limit produces a new series of excitonic 
dipole-dipole interactions that cannot be characterized by Förster's theory, but rather through molecular exciton theory.

Molecular exciton theory was developed by Alexander Davydov in $1948^{46,47}$ and Michael Kasha in $1963^{48,49}$ to explore the quantum mechanical basis for optical phenomena that result from intermolecular excitonic interactions at length scales below that of FRET $(\sim 0.2-2 \mathrm{~nm})$. Molecular exciton theory permits the development of a theoretical explanation for various optical phenomena that result from the aggregation of optically active dyes. Aggregates can be formed through either self-aggregation or induced aggregation, both of which have been observed experimentally. ${ }^{50,51}$ Typically, self-aggregation of dyes occurs spontaneously within a solution, driven by $\pi-\pi$ stacking and Van der Waals interactions. In contrast, the aggregation of some dye molecules must be promoted through the addition of ionic salts or a substrate such as DNA. ${ }^{52-56}$ Specifically, dyes can be incorporated into a DNA-templated system via four attachment schemes: (i) intercalation within the minor or major grooves of the helix, (ii) intercalation between base-pairs, (iii) substitution of a base with a non-nucleosidic molecule, or (iv) covalently tethering the dye onto a DNA strand using a carbon linker. ${ }^{54,57}$ Following Frenkel's exciton model, ${ }^{58}$ below I describe the phenomenological manifestation of exciton delocalization. Assuming a two-dye aggregate, or dimer, containing a single exciton, the wavefunction, $\varphi$, describing the superposition of the exciton can be expressed in the general form:

$$
|\varphi\rangle=\alpha|1\rangle+\beta|2\rangle
$$

where $\alpha$ and $\beta$ are complex numbers whose norms squared must sum to 1 given by: 


$$
|\alpha|^{2}+|\beta|^{2}=1
$$

Here, $|\alpha|^{2}$ represents the probability of finding the exciton on dye 1 and $|\beta|^{2}$ represents the probability of finding the exciton on dye 2 .

The energy eigenstates of the system can be obtained by solving the eigenvectoreigenvalue problem:

$$
H|\epsilon\rangle=\epsilon|\epsilon\rangle .
$$

$H$ is the Hamiltonian of the system, $\epsilon$ is the energy, and $|\epsilon\rangle$ is the corresponding eigenstate that satisfies the equation. Following from this, the Hamiltonian for the twodye, one-exciton system can be written as:

$$
H=E(|1\rangle\langle 1|+| 2\rangle\langle 2|)+J(|1\rangle\langle 2|+| 2\rangle\langle 1|),
$$

where $E$ is the energy of the absorbance peak maximum and $J$ is the dipole-dipole coupling energy (also known as the exchange energy) between the transition dipoles of dye 1 and dye 2 . Owing to the proximity with which dyes must be positioned relative to each other $(\sim 0.2-2 \mathrm{~nm})$, the coupling strength $(J)$ of exciton delocalization far surpasses that of FRET, and can be described mathematically by:

$$
J=\frac{1}{4 \pi \epsilon}\left(\frac{\boldsymbol{\mu}_{1} \cdot \boldsymbol{\mu}_{2}}{|\boldsymbol{R}|^{3}}-3 \frac{\left(\boldsymbol{R} \cdot \boldsymbol{\mu}_{1}\right)\left(\boldsymbol{R} \cdot \boldsymbol{\mu}_{2}\right)}{|\boldsymbol{R}|^{5}}\right),
$$

where $\boldsymbol{\mu}_{1}$ and $\boldsymbol{\mu}_{2}$ denote the transition dipoles of dye 1 and dye 2, respectively, $\boldsymbol{R}$ represents the vector connecting the two dipoles, and $\epsilon$ is the vacuum permittivity. Note that Eq. (1.9) is only valid when the size of the dye molecule is small compared to the distance between the molecules, such that a dipole approximation can be made. Given the symmetry of the system, the Hamiltonian can be easily solved and conceptualized as coupled harmonic oscillators that have two modes of oscillation, namely: symmetric $\left(\left|E_{S}\right\rangle\right)$ and anti-symmetric $\left(\left|E_{A}\right\rangle\right)$ energy modes expressed as: 


$$
\begin{aligned}
& \left|E_{S}\right\rangle=\frac{1}{\sqrt{2}}[|1\rangle+|2\rangle], \\
& \left|E_{A}\right\rangle=\frac{1}{\sqrt{2}}[|1\rangle-|2\rangle],
\end{aligned}
$$

where $|1\rangle$ and $|2\rangle$ represent dyes 1 and 2, respectively. Due to these two modes of coupling, the excited energy states of the dyes result in band splitting (i.e., Davydov splitting). Substituting into Eq. (1.8), we arrive at:

$$
\begin{aligned}
& E_{S}=E+J, \\
& E_{A}=E-J .
\end{aligned}
$$

Since $J$ can be positive or negative, either $E_{S}$ or $E_{A}$ can be the higher energy state of the two one-exciton excited-state energies, depending upon the sign of $J$, and Davydov Splitting can be expressed as:

$$
\left|E_{S}-E_{A}\right|=2|J|
$$

More generally, moving to larger aggregates beyond dimers, it should be noted that for $n$ dyes coupled together there will be $n$ distinct energy eigenstates.

Given a dimer system composed of two identical dyes and analyzing the possible arrangements in which the transition dipole moments can arrange, we are presented with three cases: (i) head-to-tail or antiparallel (Figure 1.6a), (ii) parallel (Figure 1.6b), and (iii) oblique (Figure 1.6c). Note that a single dye is termed a monomer, a pair of dyes a dimer, a triplet of dyes a trimer, etc.

To determine which energy state transitions are optically allowed or forbidden for each case, we simplify Eq. (1.9) as:

$$
J=\frac{J_{0}}{R^{3}}\left(\boldsymbol{\mu}_{1} \cdot \boldsymbol{\mu}_{2}-3\left(\boldsymbol{\mu}_{12} \cdot \boldsymbol{\mu}_{1}\right)\left(\boldsymbol{\mu}_{12} \cdot \boldsymbol{\mu}_{2}\right),\right.
$$

where $\boldsymbol{\mu}_{\mathbf{1 2}}$ is the dot product of $\boldsymbol{\mu}_{\mathbf{1}}$ and $\boldsymbol{\mu}_{\mathbf{2}}$ given as a unit vector in which its tail is located at the center of dye 1 and its head is located at the center of dye 2 , and $J_{0}$ is a 
constant. Additionally, definitions of general terms are required to express the interaction of an applied electromagnetic field (i.e., incoming photon) on an electric dipole (i.e., dye aggregate), using Fermi’s golden rule: ${ }^{59}$

$$
\begin{aligned}
& \Gamma_{G \rightarrow E_{S}} \propto \frac{\mu^{2}}{2}\left|\left(\boldsymbol{\mu}_{\mathbf{1}}+\boldsymbol{\mu}_{2}\right) \cdot \boldsymbol{E}\right|^{2}, \\
& \Gamma_{G \rightarrow E_{A}} \propto \frac{\mu^{2}}{2}\left|\left(\boldsymbol{\mu}_{\mathbf{1}}-\boldsymbol{\mu}_{\mathbf{2}}\right) \cdot \boldsymbol{E}\right|^{2},
\end{aligned}
$$

where $\Gamma_{G \rightarrow E_{S, A}}$ is the transition rate from the ground state $(G)$ to the symmetric $\left(E_{S}\right)$ and anti-symmetric $\left(E_{A}\right)$ states, respectively. Now, we consider all 3 cases:

CASE 1: Head-to-tail, $\boldsymbol{\mu}_{\mathbf{1 2}}=\boldsymbol{\mu}_{\mathbf{1}}=\boldsymbol{\mu}_{\mathbf{2}}=\boldsymbol{\mu}$. Substituting into Eq. (1.13), we find:

$$
J=-\frac{2 J_{0}}{R^{3}}
$$

and hence the coupling constant, $J$, is negative. Accordingly, from Eq. (1.11), we can see that $E_{S}$ is the lower energy state, and $E_{A}$ is the higher energy state. Applying an electromagnetic field, we find that:

$$
\begin{aligned}
& \Gamma_{G \rightarrow E_{S}} \propto 2 \mu^{2}|\boldsymbol{n} \cdot \boldsymbol{E}|^{2}, \\
& \Gamma_{G \rightarrow E_{A}} \propto 0 .
\end{aligned}
$$

Thus, there is an optically allowed energy transition from the ground state to the exciton in its lower energy symmetric state and an optically forbidden energy transition to the higher energy anti-symmetric state, as illustrated in Figure 1.6a.

CASE 2: Parallel, $\boldsymbol{\mu}_{\mathbf{1}}=\boldsymbol{\mu}_{\mathbf{2}}=\boldsymbol{\mu}$. Substituting into Eq. (1.13), we find:

$$
J=\frac{J_{0}}{R^{3}}
$$


and hence $J$ is positive. From Eq. (1.11), we can see that $E_{S}$ is now the higher energy state, and $E_{A}$ is the lower energy state. Applying an electromagnetic field to the dipole:

$$
\begin{aligned}
& \Gamma_{G \rightarrow E_{S}} \propto 2 \mu^{2}|\boldsymbol{n} \cdot \boldsymbol{E}|^{2}, \\
& \Gamma_{G \rightarrow E_{A}} \propto 0 .
\end{aligned}
$$

Thus the optically driven transition to the higher energy symmetric state is allowed, while the lower energy state has a forbidden transition, as illustrated in Figure $1.6 \mathrm{~b}$.

$$
\text { CASE 3: Oblique, } \boldsymbol{\mu}_{\mathbf{1}}=\cos \left(\frac{\theta}{2}\right) \hat{\boldsymbol{\imath}}+\sin \left(\frac{\theta}{2}\right) \hat{\boldsymbol{\jmath}} \text { and } \boldsymbol{\mu}_{\mathbf{2}}=\cos \left(\frac{\theta}{2}\right) \hat{\boldsymbol{\imath}}-\sin \left(\frac{\theta}{2}\right) \hat{\boldsymbol{\jmath}} \text {. }
$$

Substituting into Eq. (1.13), we find:

$$
J=\frac{J_{0}}{R^{3}} \cos (\theta),
$$

in which $J$ is dependent on $\theta$ for $0^{\circ}<\theta<180^{\circ}$, with $J$ positive for $0^{\circ}<\theta<$ $90^{\circ}$ and negative for $90^{\circ}<\theta<180^{\circ}$,. From Eq. (1.11), we find:

$$
\begin{aligned}
& E_{S}=E+\frac{J_{0}}{R^{3}} \cos (\theta), \\
& E_{A}=E-\frac{J_{0}}{R^{3}} \cos (\theta) .
\end{aligned}
$$

Applying an electromagnetic field to the system gives:

$$
\begin{aligned}
& \Gamma_{G \rightarrow E_{S}} \propto \mu^{2} \cos ^{2}\left(\frac{\theta}{2}\right) E_{x}{ }^{2}, \\
& \Gamma_{G \rightarrow E_{A}} \propto \mu^{2} \sin ^{2}\left(\frac{\theta}{2}\right) E_{y}{ }^{2},
\end{aligned}
$$

and thus, optical transitions to both excited energy states are allowed, as shown in Figure 1.6c. 

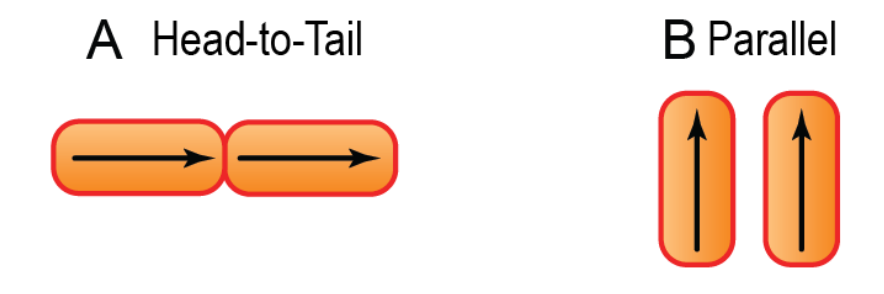

C Oblique
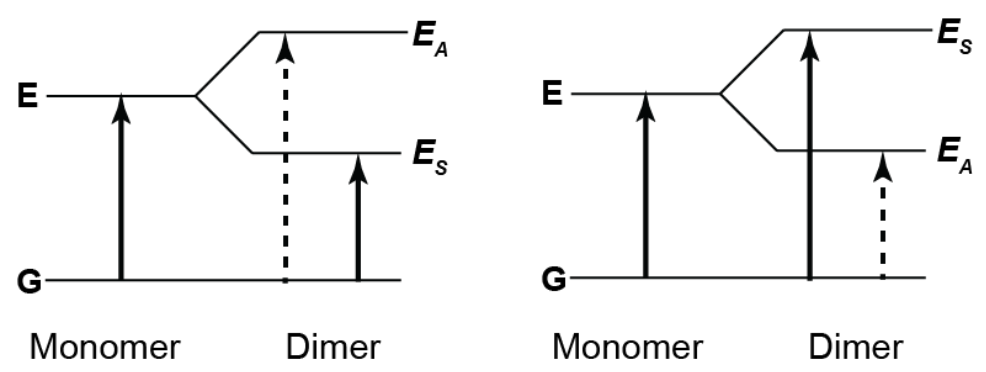

Monomer Dimer
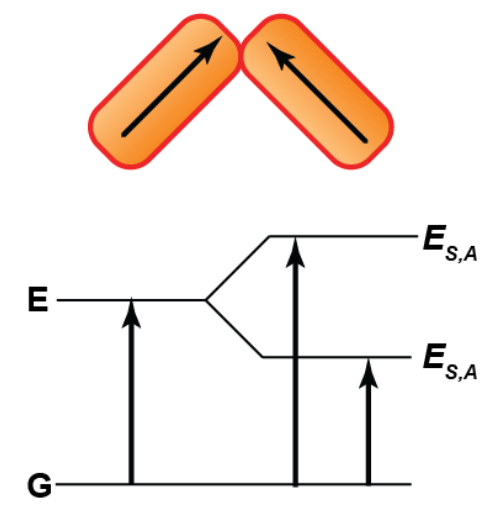

Monomer Dimer

Figure 1.6. Energy diagram illustrating the effects of dipole-dipole coupling between molecular dyes, resulting in splitting of the excited energy states. Allowed (solid black arrows) and forbidden (dashed black arrows) optical electronic state transitions are highly dependent on the relative geometrical orientation of the dyes, which can be categorized as (a) head-to-tail (anit-parallel), (b) parallel, or (c) oblique stacking arrangements.

It should be noted that for simplicity, the theory presented above does not include vibronic effects, which are necessary for making accurate experimental predications. Kühn, Renger, and May have developed theoretical work with vibronic effects taken into account, which forms the basis for the KRM theoretical model presented in Chapters 2 and $3 .{ }^{60}$ Experimentally, molecular exciton theory has been found to accurately predict the preferential stacking arrangement and can be characterized using optical techniques, including absorbance, fluorescence, and circular dichroism (CD) spectroscopy. Depending on the stacking arrangement and strength of exciton coupling, the spectral signatures of exciton delocalization can be drastically different. For instance, Jaggregates, which were first observed by Jelley ${ }^{50,51}$ and Scheibe ${ }^{61-63}$ and are stacked in a head-to-tail arrangement (Figure 1.6a), exhibit a bathochromic (i.e., red) spectral shift in the absorbance maximum and a near resonant fluorescence emission that gives rise to a small Stokes shift and a high-intensity, sharp emission peak. The unique optical 
properties of J-aggregates have been harnessed for the development of nonlinear optical devices, light-harvesting devices, and biological probes. ${ }^{54,64-68}$ Conversely, H-aggregates, which stack in a parallel fashion (Figure 1.6b), are typically used as biological markers owing to a significantly reduced fluorescence emission that arises from the optically forbidden lowest energy transition. Finally, oblique aggregates (Figure 1.6c) are stacked at an angle and produce a band (Davydov) splitting of the absorbance spectrum that reflects a combination of both $\mathrm{J}$ - and $\mathrm{H}$-aggregate spectral properties. Other spectral signatures of exciton delocalization include exchange narrowing, ${ }^{69,70}$ resonance fluorescence, ${ }^{71}$ Dicke supperradiance,,${ }^{72,73}$ superquenching,,${ }^{74}$ superfluorescence, ${ }^{75,76}$ and circular dichroism, ${ }^{67,77,78}$ each of which are each illustrated in Figure 1.7.
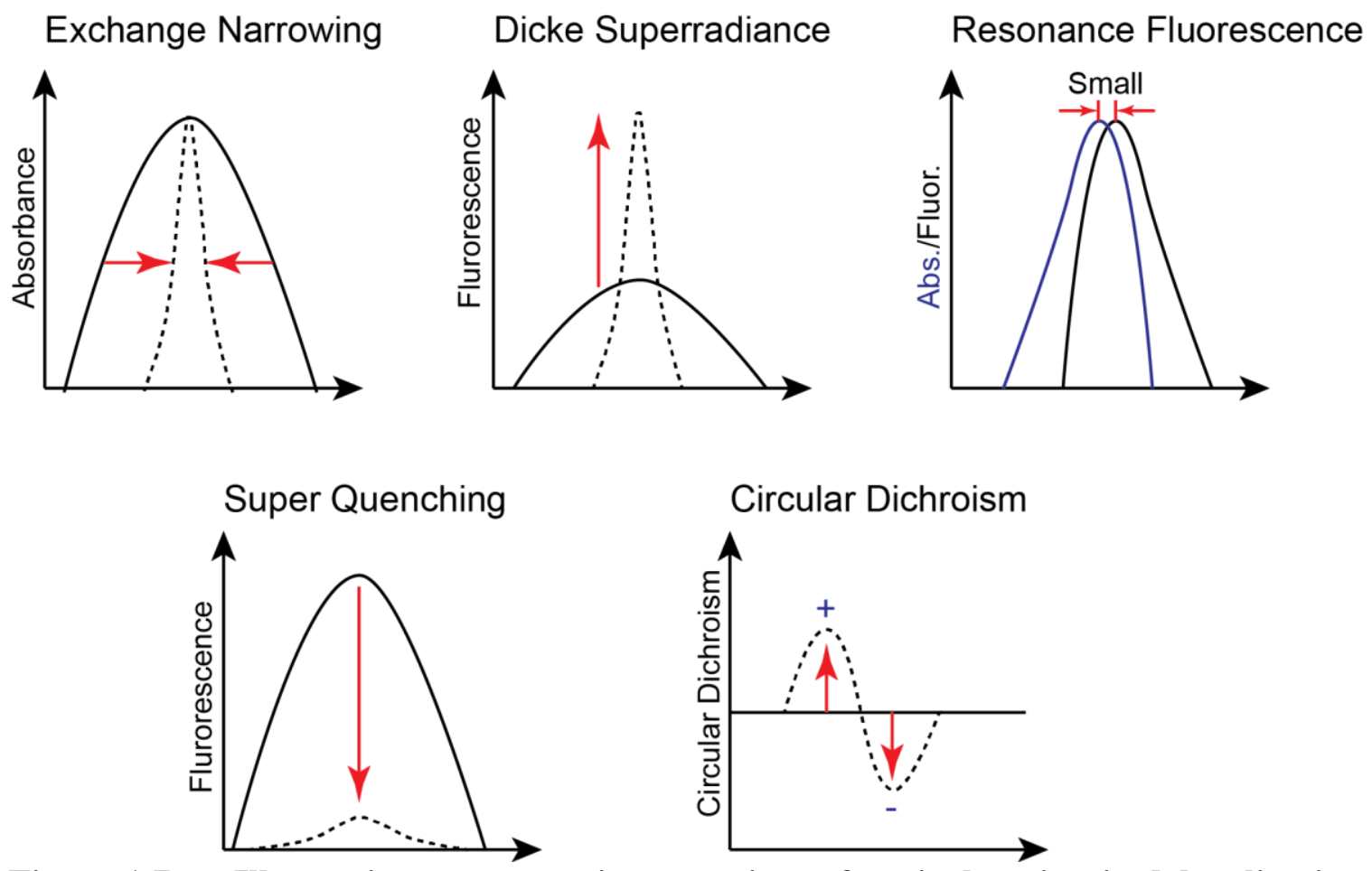

Figure 1.7. Illustrations representing a variety of optical excitonic delocalization phenomenon.

The purpose of this dissertation is to leverage the programmability and functionality of DNA to proximally position molecular dyes into aggregate 
configurations that facilitate exciton delocalization, and optically characterize the dyeDNA constructs to reveal signatures of excitonic coupling and identify the dye stacking arrangements. By determining the preferential stacking arrangements of the aggregates within the dye-DNA construct, we hope to be able to understand the mechanisms that drive aggregation and thus control the aggregation. Accordingly, by controlling the stacking arrangements, we can engineer the optical properties of the aggregate to develop the quantum devices necessary for quantum logic operations and thus provide a path towards room temperature universal quantum computing.

Previous work relating to DNA-templated dye aggregates and exciton delocalization has focused primarily on identifying amenable dye-to-DNA functionalization schemes that enable either J- or H-aggregates for optical reporting applications. ${ }^{54,57,79} \mathrm{~A}$ few studies have reported on Davydov splitting ${ }^{67}$ and dynamic structures that are capable of switching between electronically isolated molecular dyes to excitonically-coupled aggregates; ${ }^{52}$ however, to our knowledge, there has not been any previous work that demonstrates large Davydov splitting (>200 meV) or structures that can be tuned between primarily J-like aggregates and primarily H-like aggregates.

The bulk of this dissertation is composed of two papers, both of which have been published for publication. ${ }^{80,81}$ Chapter Two focuses on understanding the basic design specifications and metrics necessary for inducing, maintaining, and optimizing excitonic quantum coherence in DNA-templated molecular aggregates. Specifically, the chapter investigates a simple dye dimer and the effects of altering particular external parameters (e.g., temperature, salt concentration, and DNA concentration) on the system. Results indicated that systems with low salt and DNA concentrations favored J-aggregate type 
behavior. Increasing the salt and/or DNA concentration promoted higher-ordered aggregation of the duplexed dimers into a tetramer with predominantly $\mathrm{H}$-aggregate characteristics through formation of a mobile 4-arm (Holliday) junction via a strandmediated displacement reaction. This salt and/or DNA concentration dependence enables dynamic and discrete control over the aggregates, their optical properties, and resultant quantum coherent behavior. Chapter Three examines a structurally more complex system comprised of a 4-arm junction (i.e., Holliday Junction) that provides a simple structure for modulating the number of dyes constituting the aggregate (i.e., dimer, trimer, or tetramer). It was found that the 4-arm DNA junction improved the excitonic delocalization behavior as evidenced by a larger Davydov splitting in the absorbance and $\mathrm{CD}$ spectra and greater fluorescence suppression, which is hypothesized to be a result of the greater structural rigidity afforded by the immobile 4-arm junction. Additionally, by exploiting the unique optical properties of the tetramer (i.e., large Davydov splitting and superfluorescence suppression) we demonstrate two modes of optical detection, using both absorbance and fluorescence characterization techniques. Finally, Chapter 4 summarizes the results of Chapters Two and Three, offering possible directions for future work. 


\section{REFERENCES}

[1] Feynman, R. P., Simulating Physics with Computers. International Journal of Theoretical Physics 1982, 21 (6-7), 467-488.

[2] Benioff, P., The Computer as a Physical System - A Microscopic QuantumMechanical Hamiltonian Model of Computers as Represented by Turing-Machines. Journal of Statistical Physics 1980, 22 (5), 563-591.

[3] Cho, A., Computer Science: Quantum or Not, Controversial Computer Yields No Speedup. Science 2014, 344 (6190), 1330-1331.

[4] Ronnow, T. F.; Wang, Z. H.; Job, J.; Boixo, S.; Isakov, S. V.; Wecker, D.; Martinis, J. M.; Lidar, D. A.; Troyer, M., Defining and Detecting Quantum Speedup. Science 2014, 345 (6195), 420-424.

[5] Meet D-Wave: Our Vision and History. (accessed February).

[6] Briggs, J. S.; Eisfeld, A., Equivalence of Quantum and Classical Coherence in Electronic Energy Transfer. Physical Review E 2011, 83 (5).

[7] Dawlaty, J. M.; Ishizaki, A.; De, A. K.; Fleming, G. R., Microscopic Quantum Coherence in a Photosynthetic-Light-Harvesting Antenna. Philosophical Transactions of the Royal Society a-Mathematical Physical and Engineering Sciences 2012, 370 (1972), 3672-3691.

[8] Eisfeld, A.; Briggs, J. S., Classical Master Equation for Excitonic Transport Under the Influence of an Environment. Physical Review E 2012, 85 (4).

[9] Fidler, A. F.; Caram, J. R.; Hayes, D.; Engel, G. S., Towards a Coherent Picture of Excitonic Coherence in the Fenna-Matthews-Olson Complex. Journal of Physics B-Atomic Molecular and Optical Physics 2012, 45 (15).

[10] Larson, C. R.; Seng, C. O.; Lauman, L.; Matthies, H. J.; Wen, J. Z.; Blankenship, R. E.; Allen, J. P., The Three-Dimensional Structure of the FMO Protein from Pelodictyon phaeum and the Implications for Energy Transfer. Photosynthesis Research 2011, 107 (2), 139-150. 
[11] Fenna, R. E.; Matthews, B. W., Chlorophyll Arrangement in a Bacteriochlorophyll Protein from Chlorobium-limicola. Nature 1975, 258 (5536), 573-577.

[12] Collini, E., Spectroscopic Signatures of Quantum-Coherent Energy Transfer. Chemical Society Reviews 2013, 42 (12), 4932-4947.

[13] Engel, G. S.; Calhoun, T. R.; Read, E. L.; Ahn, T.-K.; Mancal, T.; Cheng, Y.-C.; Blankenship, R. E.; Fleming, G. R., Evidence for Wavelike Energy Transfer through Quantum Coherence in Photosynthetic Systems. Nature 2007, 446 (7137), 782-786.

[14] Engel, G. S., Quantum Coherence in Photosynthesis. Procedia Chemistry 2011, 3, 222-231.

[15] Ishizaki, A.; Fleming, G. R., Quantum Coherence in Photosynthetic Light Harvesting. In Annual Review of Condensed Matter Physics, Vol 3, Langer, J. S., Ed. 2012; Vol. 3, pp 333-361.

[16] Nalbach, P.; Thorwart, M., Quantum Coherence and Entanglement in Photosynthetic Light-Harvesting Complexes. In Quantum Efficiency in Complex Systems, Pt I: Biomolecular Systems, Weber, E. R.; Thorwart, M.; Wurfel, U., Eds. 2010; Vol. 83, pp 39-75.

[17] Scholes, G. D.; Fleming, G. R.; Olaya-Castro, A.; van Grondelle, R., Lessons From Nature about Solar Light Harvesting. Nature Chemistry 2011, 3 (10), 763-774.

[18] Scholes, G. D., Quantum Biology: Coherence in Photosynthesis. Nature Physics 2011, 7 (6), 448-449.

[19] Kallenbach, N. R.; Ma, R. I.; Seeman, N. C., An Immobile Nucliec-Acid Junction Contructed From Oligonucleotides. Nature 1983, 305 (5937), 829-831.

[20] Seeman, N. C., Nucleic-Acid Junction and Lattices. Journal of Theoretical Biology 1982, 99 (2), 237-247.

[21] Seeman, N. C.; Kallenbach, N. R., Optimal-Design of Immobile and Semi-mobile Nucleic-Acid Junctions. Biophysical Journal 1982, 37 (2), A93-A93.

[22] Seeman, N. C.; Kallenbach, N. R., Design of Immobile Nucliec-Acid Junctions. Biophysical Journal 1983, 44 (2), 201-209.

[23] Seeman, N. C., DNA in a Material World. Nature 2003, 421 (6921), 427-431.

[24] Winfree, E.; Liu, F. R.; Wenzler, L. A.; Seeman, N. C., Design and Self-Assembly of Two-Dimensional DNA Crystals. Nature 1998, 394 (6693), 539-544. 
[25] Wikipedia: DNA. https://en.wikipedia.org/wiki/DNA (accessed August 20).

[26] Rothemund, P., Design of DNA Origami. Proceedings of the IEEE/ACM International Conference in Computer-Aided Design, Pasadena, CA, 2005, 471.

[27] Arbona, J. M.; Elezgaray, J.; Aime, J. P., Modelling the Folding of DNA Origami. Europhysics Letters 2012, 100 (2), 6.

[28] Rothemund, P. W. K.; Andersen, E. S., Nanotechnology: The Importance of Being Modular. Nature 2012, 485 (7400), 584-585.

[29] Zadegan, R. M.; Norton, M. L., Structural DNA Nanotechnology: From Design to Applications. International Journal of Molecular Sciences 2012, 13 (6), 7149-7162.

[30] Rothemund, P. W. K., Folding DNA to Create Nanoscale Shapes and Patterns. Nature 2006, 440 (7082), 297-302.

[31] Dietz, H.; Douglas, S. M.; Shih, W. M., Folding DNA into Twisted and Curved Nanoscale Shapes. Science 2009, 325 (5941), 725-730.

[32] Douglas, S. M.; Marblestone, A. H.; Teerapittayanon, S.; Vazquez, A.; Church, G. M.; Shih, W. M., Rapid Prototyping of 3D DNA-Origami Shapes with caDNAno. Nucleic Acids Research 2009, 37 (15), 5001-5006.

[33] Douglas, S. M.; Dietz, H.; Liedl, T.; Hogberg, B.; Graf, F.; Shih, W. M., Selfassembly of DNA into Nanoscale Three-dimensional Shapes. Nature 2009, 459 (7245), 414-418.

[34] Zadeh, J. N.; Steenberg, C. D.; Bois, J. S.; Wolfe, B. R.; Pierce, M. B.; Khan, A. R.; Dirks, R. M.; Pierce, N. A., NUPACK: Analysis and Design of Nucleic Acid Systems. Journal of Computational Chemistry 2011, 32 (1), 170-173.

[35] Dirks, R. M.; Pierce, N. A., An Algorithm for Computing Nucleic Acid Basepairing Probabilities Including Pseudoknots. Journal of Computational Chemistry 2004, 25 (10), 1295-1304.

[36] Dirks, R. M.; Bois, J. S.; Schaeffer, J. M.; Winfree, E.; Pierce, N. A., Thermodynamic Analysis of Interacting Nucleic Acid Strands. Siam Review 2007, 49 (1), 65-88.

[37] Li, Z.; Wang, L.; Yan, H.; Liu, Y., Effect of DNA Hairpin Loops on the Twist of Planar DNA Origami Tiles. Langmuir 2012, 28 (4), 1959-1965. 
[38] Ke, Y. G.; Ong, L. L.; Shih, W. M.; Yin, P., Three-Dimensional Structures SelfAssembled from DNA Bricks. Science 2012, 338 (6111), 1177-1183.

[39] Wei, B.; Dai, M. J.; Yin, P., Complex Shapes Self-assembled from Single-stranded DNA Tiles. Nature 2012, 485 (7400), 623-+.

[40] Forster, T., Intermolecular Energy Migration and Fluorescence. Annals of Physics 1948, 437, 55-75.

[41] Dietrich, A.; Buschmann, V.; Muller, C.; Sauer, M., Fluorescence Resonance Energy Transfer (FRET) and Competing Processes in Donor-Acceptor Substituted DNA Strands: A Comparative Study of Ensemble and Single-Molecule Data. Reviews in Molecular Biotechnology, 2002; Vol. 82, pp 211-231.

[42] Clegg, R. M., Forster Resonance Energy Transfer - FRET What is It, Why do It, and How It's Done. Laboratory Techniques in Biochemistry and Molecular Biology, 2009; $33,1-57$.

[43] Van der Meer, B. W., Kappa-squared: From Nuisance to New Sense. Reviews in Molecular Biotechnology 2002, 82, 181-196.

[44] Van der Meer, B., FRET - Forster Resonance Energy Transfer: From theory to applications. 1st edition; Wiley-VCH: Weinheim, 2013.

[45] Didenko, V. V., DNA Probes using Fluorescence Resonance Energy Transfer (FRET): Designs and Applications. Biotechniques 2001, 31 (5), 1106-+.

[46] Davydov, A. S., Theory of Absorption Spectra of Molecular Crystals. Institute of Physics, Academy of Sciences of Ukrainian SSR: Kyiv, Ukraine, 1948; 18.

[47] Davydov, A. S., The Theory of Molecular Excitons. Soviet Physics 1964, 82 (3-4), 145-178.

[48] Kasha, M., Energy Transfer Mechanisms and Molecular Exciton Model for Moelcular Aggregates. Radiation Research 1963, 20 (1), 55-\&.

[49] Kasha, M., The Exciton Model in Molecular Spectroscopy. Pure and Applied Chemistry 1965, 11 (3-4), 371.

[50] Jelley, E. E., Spectral Absorption and Fluorescence of Dyes in the Molecular State. Nature 1936, 138, 1009-1010.

[51] Jelley, E. E., Molecular, Nematic and Crystal States of I: I-Diethyl-Cyanine Chloride. Nature 1937, 139 (3519), 631-632. 
[52] Asanuma, H.; Shirasuka, K.; Takarada, T.; Kashida, H.; Komiyama, M., DNA-Dye Conjugates for Controllable $\mathrm{H}^{*}$ Aggregation. Journal of the American Chemical Society 2003, 125 (8), 2217-2223.

[53] Ruedas-Rama, M. J.; Alvarez-Pez, J. M.; Orte, A., Formation of Stable BOBO-3 H-Aggregate Complexes Hinders DNA Hybridization. Journal of Physical Chemistry B 2010, 114 (27), 9063-9071.

[54] Asanuma, H.; Fujii, T.; Kato, T.; Kashida, H., Coherent Interactions of Dyes Assembled on DNA. Journal of Photochemistry and Photobiology C-Photochemistry Reviews 2012, 13 (2), 124-135.

[55] Mooi, S. M.; Keller, S. N.; Heyne, B., Forcing Aggregation of Cyanine Dyes with Salts: A Fine Line between Dimers and Higher Ordered Aggregates. Langmuir 2014, 30 (32), 9654-9662.

[56] Ruedas-Rama, M. J.; Orte, A.; Martin-Domingo, M. C.; Castello, F.; Talavera, E. M.; Alvarez-Pez, J. M., Interaction of YOYO-3 with Different DNA Templates to Form H-Aggregates. Journal of Physical Chemistry B 2014, 118 (23), 6098-6106.

[57] Kashida, H.; Asanuma, H., Preparation of Supramolecular Chromophoric Assemblies using a DNA Duplex. Physical Chemistry Chemical Physics 2012, 14 (20), 7196-7204.

[58] Frenkel, J., On the Transformation of Light into Heat in Solids. II. Physical Review 1931, 37 (10), 1276-1294.

[59] Merzbacher, E., Quantum Mechanics. 3rd ed.; Hamilton Printing Company: United State of America, 1998.

[60] Kühn, O.; Renger, T.; May, V., Theory of exciton-vibrational dynamics in molecular dimers. Chemical Physics 1996, 204 (1), 99-114.

[61] Scheibe, G., Über die Veränderlichkeit der Absorptionsspektren in Lösungen und die Nebenvalenzen als ihre Ursache. Angewandte Chemie 1937, 50.11, 212-219.

[62] Scheibe, G.; Kandler, L.; Ecker, H., Polymerisation and Polymere Adsorption as a Cause of Novel Absorption Bands of Organic Pigments. Naturwissenschaften 1937, 25 , 75-75.

[63] Scheibe, G.; Mareis, A.; Ecker, H., The Reversible Polymerisation as a Cause of Unusual Absorption Bands III. Naturwissenschaften 1937, 25, 474-475. 
[64] Wang, M. M.; Silva, G. L.; Armitage, B. A., DNA-templated Formation of a Helical Cyanine Dye J-aggregate. Journal of the American Chemical Society 2000, 122 (41), 99779986.

[65] Eisfeld, A.; Briggs, J. S., The J-band of Organic Dyes: Lineshape and Coherence Length. Chemical Physics 2002, 281 (1), 61-70.

[66] Wuerthner, F.; Kaiser, T. E.; Saha-Moeller, C. R., J-Aggregates: From Serendipitous Discovery to Supramolecular Engineering of Functional Dye Materials. Angewandte Chemie-International Edition 2011, 50 (15), 3376-3410.

[67] Markova, L. I.; Malinovskii, V. L.; Patsenker, L. D.; Haner, R., J- vs. H-type Assembly: Pentamethine Cyanine (Cy5) as a Near-IR Chiroptical Reporter. Chemical Communications 2013, 49 (46), 5298-5300.

[68] Li, Z. a.; Mukhopadhyay, S.; Jang, S.-H.; Bredas, J.-L.; Jen, A. K. Y., Supramolecular Assembly of Complementary Cyanine Salt J-Aggregates. Journal of the American Chemical Society 2015, 137 (37), 11920-11923.

[69] Knapp, E. W., Lineshapes of Molecular Aggregates - Exchnage Narrowing and Intersite Correlation. Chemical Physics 1984, 85 (1), 73-82.

[70] Walczak, P. B.; Eisfeld, A.; Briggs, J. S., Exchange Narrowing of the J Band of Molecular Dye Aggregates. Journal of Chemical Physics 2008, 128 (4).

[71] Marcus, R. J.; Haugen, G. R., Resonance Fluorescence in Chlorophyll a Solutions. Photochemistry and Photobiology 1965, 4, 183-192.

[72] Monshouwer, R.; Abrahamsson, M.; vanMourik, F.; vanGrondelle, R., Superradiance and Exciton Delocalization in Bacterial Photosynthetic Light-harvesting Systems. Journal of Physical Chemistry B 1997, 101 (37), 7241-7248.

[73] Dicke, R. H., Coherence in Spontaneoous Radiation Processes. Physical Review 1954, 93 (1), 99-110.

[74] Jones, R. M.; Bergstedt, T. S.; Buscher, C. T.; McBranch, D.; Whitten, D., Superquenching and Its Applications in J-aggregated Cyanine Polymers. Langmuir 2001, 17 (9), 2568-2571.

[75] Bonifacio, R.; Lugiato, L. A., Cooperative Radiation Processes in 2-level Systems - Superfluorescence. Physical Review A 1975, 11 (5), 1507-1521. 
[76] Bonifacio, R.; Lugiato, L. A., Cooperative Radiation Processes in 2-level Systems - Superfluorescence: 2. Physical Review A 1975, 12 (2), 587-598.

[77] Person, R. V.; Monde, K.; Humpf, H. U.; Berova, N.; Nakanishi, K., A New Approach in Exciton-coupled Circular-Dichroism (ECCD) - Insertion of an Auxillary Sterogenic Center. Chirality 1995, 7 (3), 128-135.

[78] Eisfeld, A.; Kniprath, R.; Briggs, J. S., Theory of the Absorption and Circular Dichroism Spectra of Helical Molecular Aggregates. Journal of Chemical Physics 2007, 126 (10).

[79] Armitage, B. A., Cyanine Dye-DNA Interactions: Intercalation, Groove Binding, and Aggregation. DNA Binders and Related Subjects 2005, 253, 55-76.

[80] Cannon, B. L.; Kellis, D. L.; Patten, L. K.; Davis, P. H.; Lee, J.; Graugnard, E.; Yurke, B.; Knowlton, W. B., Coherent Exciton Delocalization in a Two-State DNATemplated Dye Aggregate System. Journal of Physical Chemistry A 2017, 121 (37), 69056916.

[81] Cannon, B. L.; Patten, L. K.; Kellis, D. L.; Davis, P. H.; Lee, J.; Graugnard, E.; Yurke, B.; Knowlton, W. B., Large Davydov Splitting and Strong Flurescence Suppression: An Investigation of Exciton Delocalization in DNA-Templated Holliday Junction Dye Aggregates. The Journal of Physical Chemistry A 2018, 122 (8), 2086-2095.

[82] Yurke, B.; Kuang, W., Passive Linear Nanoscale Optical and Molecular Electronics Device Synthesis from Nanoparticles. Physical Review A 2010, 81 (3). 


\section{CHAPTER TWO: COHERENT EXCITON DELOCALIZATION IN A TWO-STATE DNA-TEMPLATED DYE AGGREGATE SYSTEM}

This chapter is published by the American Chemical Society in Journal of Physical Chemistry $A$ and should be referenced appropriately.

Reference:

Cannon, B. L.; Kellis, D. L.; Patten, L. K.; Davis, P. H.; Lee, J.; Graugnard, E.; Yurke, B; Knowlton, W. B., Coherent Exciton Delocalization in a Two-State DNA-Templated Dye Aggregate System, Journal of Physical Chemistry A 2017, 121 (37), 6905-6916.

Reproduced/modified by permission of the American Chemical Society.

*This chapter includes modifications from the originally published version. 


\title{
Coherent Exciton Delocalization in a Two-State DNA-Templated Dye Aggregate System
}

\author{
Brittany L. Cannon ${ }^{\mathrm{a}}$ \\ Donald L. Kellis ${ }^{\mathrm{a}}$ \\ Lance K. Patten ${ }^{\mathrm{a}}$ \\ Paul H. Davis ${ }^{\mathrm{a}}$ \\ Jeunghoon Lee ${ }^{\mathrm{b}}$ \\ Elton Graugnard ${ }^{\mathrm{a}}$ \\ Bernard Yurke $e^{\mathrm{a}, \mathrm{c}}$ \\ William B. Knowlton ${ }^{\mathrm{a}, \mathrm{c}}$
}

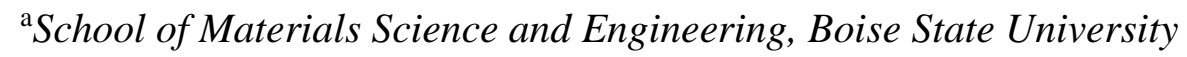

${ }^{\mathrm{b}}$ Department of Chemistry and Biochemistry, Boise State University

${ }^{\mathrm{c}}$ Department of Electrical and Computer Engineering, Boise State University

1920 University Dr., Boise ID 83725 USA. 


\begin{abstract}
Coherent exciton delocalization in dye aggregate systems gives rise to a variety of intriguing optical phenomena, including $\mathrm{J}$ - and $\mathrm{H}$-aggregate behavior and Davydov splitting. Systems that exhibit coherent exciton delocalization at room temperature are of interest for the development of artificial light-harvesting devices, colorimetric detection schemes, and quantum computers. Here, we report on a simple dye system templated by DNA that exhibits tunable optical properties. At low salt and DNA concentrations, a DNA duplex with two internally functionalized Cy5 dyes (i.e., dimer) persists and displays predominantly J-aggregate behavior. Increasing the salt and/or DNA concentrations was found to promote coupling between two of the DNA duplexes via branch migration, thus forming a four-armed junction (i.e., tetramer) with $\mathrm{H}$-aggregate behavior. This $\mathrm{H}$-tetramer aggregate exhibits a surprisingly large Davydov splitting in its absorbance spectrum that produces a visible color change of the solution from cyan to violet and gives clear evidence of coherent exciton delocalization.
\end{abstract}

\title{
2.1 Introduction
}

Coherent exciton delocalization, the process by which excitons (i.e., electron-hole pairs) spread in a wavelike manner over spatially separated molecular dyes, ${ }^{1-5}$ is an intriguing phenomenon that has attracted the interest of quantum computational theorists for the role it might play in photosynthesis. ${ }^{5-11}$ Frenkel, Davydov, and Kasha ${ }^{12-15}$ pioneered the theoretical description of exciton delocalization within molecular crystals and aggregates. Exciton delocalization in dye aggregate systems has been shown to exhibit itself in a wide variety of optical phenomena, including Dicke superradiance, ${ }^{16-18}$ Davydov splitting, ${ }^{12,13,19-21}$ and its more specific manifestations: J- 
and $\mathrm{H}$-aggregate behavior, ${ }^{22-39}$ superquenching, ${ }^{28,40}$ exchange narrowing, ${ }^{41,42}$ superfluorescence, ${ }^{43,44}$ resonance fluorescence,${ }^{45-47}$ and excitonically coupled circular dichroism (EC-CD) ${ }^{26,48}$ many of which depend strongly on the geometrical configuration of the dye aggregates. Shown in Figure 2.1, coherent coupling between two dyes results in a splitting of the excited state energy levels for which the allowed energy transitions, and thus optical properties are strikingly different depending on whether the dye molecules undergo head-to-tail stacking (referred to as J-aggregates, Figure 2.1a) or parallel stacking (referred to as H-aggregates, Figure 2.1b). In particular, J-aggregates, first observed by Jelley ${ }^{49,50}$ and Scheibe, ${ }^{51-53}$ exhibit a bathochromic (red) shift in absorbance, while H-aggregates exhibit a hypsochromic (blue) shift in absorbance relative to the monomeric dye. J-aggregates have been shown to produce a nearly resonant fluorescence emission (i.e., very small Stokes shift), yielding a high-intensity, sharp emission peak that can be exploited for the development of nonlinear optical devices,${ }^{30}$ light-harvesting systems, and biological probes. ${ }^{22,54}$ Conversely, H-aggregates have been used primarily as biological markers ${ }^{35,37,55,56}$ due to a significantly reduced fluorescence emission intensity accompanied by a very large Stokes shift that arises from an optically forbidden energy transition. Intermediate geometrical configurations, termed oblique, exhibit a resonant band (Davydov) splitting of the absorbance spectrum that reflects a mixture of $\mathrm{J}$ - and $\mathrm{H}$ - aggregate optical properties resulting from the dye stacking geometry. ${ }^{20}$ 

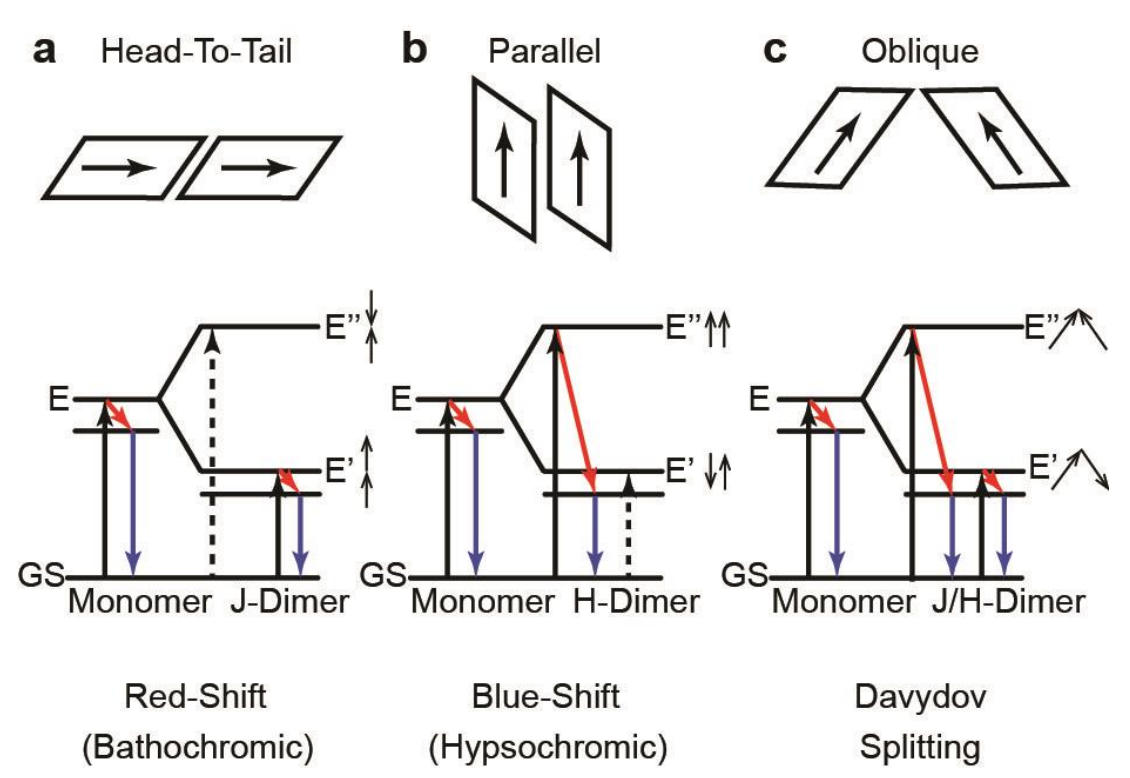

Figure 2.1. Energy diagram illustrating the different excitation and relaxation pathways possible within (a) J-dimer, (b) H-dimer, and (c) oblique systems. Allowed absorption transitions are represented as solid black arrows, while dashed arrows indicate optically forbidden transitions. Blue and red arrows signify observed emission processes and nonradiative relaxation, respectively. The small arrows adjacent to each excited energy level represent the transition dipole moment of the corresponding molecules. Adapted from Kasha with permission. ${ }^{14}$

Currently, coherent exciton delocalization is being explored in naturally occurring photosynthetic systems as a highly efficient energy transfer mechanism at ambient temperatures. ${ }^{6,10,11,57-59} \mathrm{Within}$ these light-harvesting systems, proteins organize groups of optically active dyes into aggregates with nearest-neighbor distances of 1-2 nm or less, which is necessary to induce and sustain coherence. ${ }^{59}$ Mimicking intricate protein and dye interactions found within photosynthetic light-harvesting systems to assemble dye aggregates in a manner that promotes exciton delocalization has proven difficult owing to the complexity of protein folding mechanisms and dye-protein interactions. ${ }^{8,60}$ An alternative approach is to use DNA as the biomolecular scaffold. Largely because of Watson-Crick base pairing and the linear structure of DNA oligomers, DNA assembly follows simple design rules. In addition, DNA can be easily labeled (i.e., functionalized) with an assortment of dye species using various dye attachment methods that enable 
proximal positioning of the dyes, thereby facilitating coherent interactions. ${ }^{40,61}$ Covalent attachment of dyes onto DNA oligomers ${ }^{26,48,62-65}$ enables precise control of aggregate size and specificity of the covalent attachment site as well as offers a simple method for investigating the effects of dye surroundings on dye-dye interactions and the preferred aggregation stacking arrangement. As already established by Markova et al., two cyanine-based Cy5 dyes (see section 2.7.1) covalently bound within a DNA duplex form a J-dimer while in the presence of $100 \mathrm{mM}$ sodium chloride $(\mathrm{NaCl}) .{ }^{26} \mathrm{In}$ our work, we investigate the transition of J-type to H-type aggregate constructs at high salt and/or DNA concentrations through theoretical fitting of the spectral data, gel electrophoresis, and reaction kinetics studies, which supports the coupling of two J-dimers to form an $\mathrm{H}$ tetramer by the combination of two DNA duplexes into a four-armed junction (4AJ). Strictly speaking, the DNA-templated J-dimers and H-tetramers are chiral oblique aggregates, as evidenced by our circular dichroism (CD) data, but exhibit predominantly J- or H-characteristics, respectively. Within the bulk solution, the equilibrium population ratios between the two aggregate states (i.e., J-dimer/duplex and H-tetramer/4AJ) are adjusted by varying the salt and DNA concentrations, thereby enabling control of the optical properties. In particular, for this two-state system, the large Davydov splitting $(103 \mathrm{~nm}, 336 \mathrm{meV})$ manifested in the absorption spectra gives rise to vivid color changes as a result of the large separation between the two absorption peaks, establishing a distinct and visible signature of exciton delocalization. ${ }^{66}$ Exploiting this unique DNAtemplated method for discretely switching between aggregate states and precisely controlling the optical properties of the system provides a viable platform for the 
development of excitonic based optoelectronic devices toward artificial light-harvesting systems, novel colorimetric detection schemes, and quantum computation. ${ }^{8,9}$

\subsection{Theoretical Analysis of Optical Spectra for Structural Modeling}

To extract structural information from the experimental data, theoretical absorbance and CD spectra were computed for various dye configurations and compared with the experimental spectra. The theoretical calculations proceeded by constructing a system Hamiltonian following a generalized approach guided by Kü hn, Renger, and May that considers the case of $\mathrm{N}$ dye molecules having arbitrary positions and orientations. ${ }^{67-71}$ This approach, which we will term the Kühn-Renger-May (KRM) model from here on, avoids any perturbational treatment of the exciton-vibrational coupling. The energy eigenvalues and eigenvectors of this Hamiltonian were computed on a truncated Hilbert space. The construction of the Hamiltonian and the truncation of the Hilbert space are described in section 2.7.2. Since the distance between the dyes is expected to be less than the length of the molecules, to achieve a better approximation rather than model the exciton exchange interaction as that between a pair of point dipoles, each dipole is modeled as a pair of opposite charges separated by a distance comparable to that of the length of the molecule (section 2.7.2). The optical transition probabilities were computed for each eigenstate, and smooth absorption and CD spectra were obtained by convolving the transition probability line spectra with a Gaussian line shape, thereby approximating the effect of the vibronic continuum on spectral line shape. The absorbance and CD data were simultaneously fit to match the two dominant peaks of the absorption and CD spectra and obtain a generally good qualitative agreement for the shape of the spectra. The purpose of the KRM modeling is to obtain quantitative 
information regarding the dye stacking positions and orientations. From a qualitative inspection of the experimental data, it may be apparent that the dyes do not stack strictly in head-to-tail J-aggregate or face-to-face $\mathrm{H}$-aggregate configurations, but it is not possible to determine the actual configurations that the structures will adopt.

\subsection{Kinetics and Thermodynamics of the Two-State Model}

As will be highlighted when discussing the results of the reaction kinetics and thermodynamic studies of the system, the model that best describes the data is a secondorder reaction in which two J-dimers reversibly combine to form an $\mathrm{H}$-tetramer. This model is also corroborated by the theoretical fitting of optical spectra, which shows two J-dimer duplexes that couple together to form a $4 \mathrm{AJ}$ with four dyes (i.e., a tetramer) exhibiting predominantly $\mathrm{H}$-aggregate behavior. Here, the model is described in detail.

\subsubsection{Two-State Second-Order Kinetics Reaction Model}

The system in this study can be characterized by the following isothermal chemical reaction:

$$
[\mathrm{J}]+[\mathrm{J}] \underset{k_{H \rightarrow J}}{\stackrel{k_{J \rightarrow H}}{\rightleftharpoons}}[\mathrm{H}]
$$

where [J], [H], $k_{J \rightarrow H}$, and $k_{H \rightarrow J}$ represent J-dimers, and $\mathrm{H}$-tetramer concentrations, the reaction rate constant for J-dimers combining to form $\mathrm{H}$-tetramers (i.e., forward reaction), and the reaction rate constant for $\mathrm{H}$-tetramers dissociating into pairs of J-dimers (i.e., reverse reaction), respectively. A schematic illustration of the J-dimer to H-tetramer states is given in Figure 2.2. The transition occurs via a four-way branch migration process to form a Holliday Junction-like structure with dyes located at the junction core. ${ }^{72-82}$ Figure 2.2 also indicates that, although the $\mathrm{H}$-type configuration is treated as a 
single state in the two-state reaction kinetics model, it is depicted as undergoing internal rearrangements due to breathing and restacking of the DNA bases near the Holliday junction core. These arrangements occur on $1 \mu \mathrm{s}$ to $1 \mathrm{~s}$ time scales and are fast compared to interconversion of J-dimers and H-tetramers. The internal rearrangements in Figure 2.2 are shown causing either a horizontal or vertical displacement, or separation of the dyes, resulting in the formation of dye dimers. We hypothesize that the existence of these multiple internal states (i.e., dimers that result from horizontal or vertical displacement) largely accounts for the J-aggregate like feature of the H-tetramer absorbance spectrum that appears at $665 \mathrm{~nm}$.

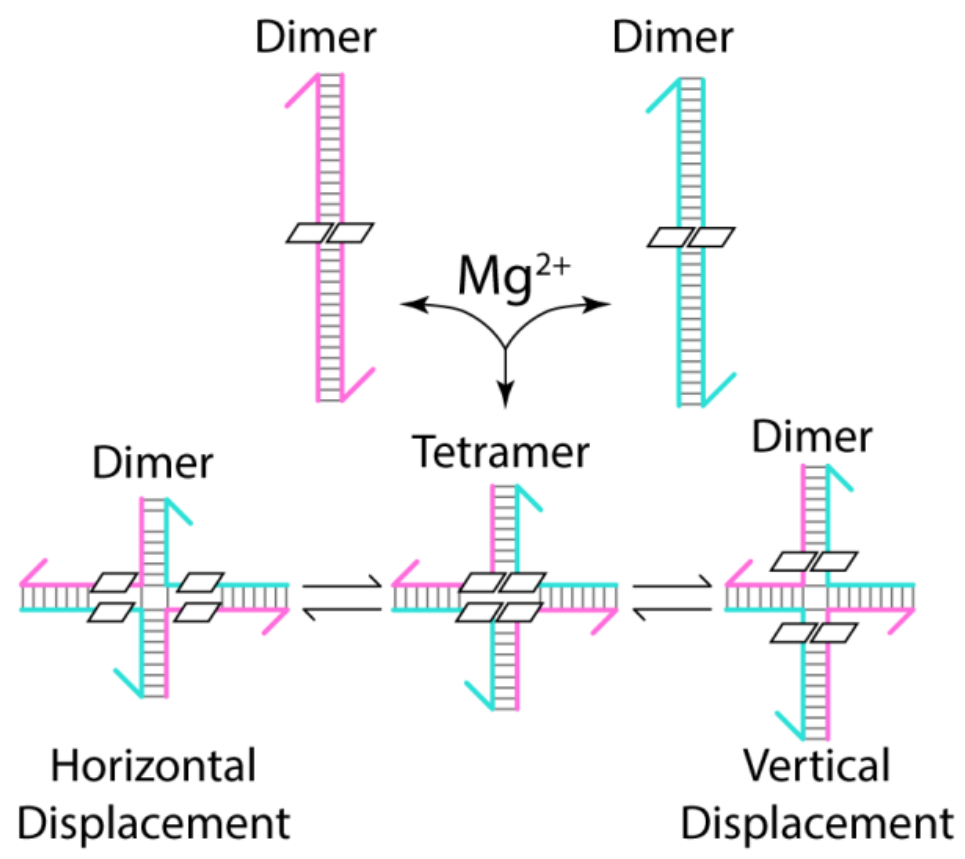

Figure 2.2. Schematic illustration of the constituents of the two-state second-order reaction kinetics model in which two J-dimers couple together to form an H-tetramer through a four-way branch migration process. Also illustrated are the internal rearrangements within the core of the H-tetramer due to DNA breathing. As shown, dye dimers result when four-way branch migration steps cause the horizontal arms to become either longer or shorter than that of the dye tetramer, where all four dyes are located at the junction core. 
Here, we derive he equations that follow from Eq. (2.1) that were used to fit the experimental kinetics and thermodynamic data. The rate equations for such a system are:

$$
\frac{\mathrm{d}[\mathrm{J}]}{\mathrm{d} t}=-2 k_{J \rightarrow H}[\mathrm{~J}]^{2}+2 k_{H \rightarrow J}[\mathrm{H}]
$$

and

$$
\frac{\mathrm{d}[\mathrm{H}]}{\mathrm{d} t}=k_{J \rightarrow H}[\mathrm{~J}]^{2}-k_{H \rightarrow J}[\mathrm{H}] .
$$

From Eqs. (2.2) and (2.3), it follows that:

$$
\frac{\mathrm{d}[\mathrm{J}]}{\mathrm{d} t}+2 \frac{\mathrm{d}[\mathrm{H}]}{\mathrm{d} t}=0 .
$$

If, at time $t=0,[\mathrm{~J}]=[\mathrm{J}]_{0}$ and $[\mathrm{H}]=[\mathrm{H}]_{0}$, then upon integrating Eq. (2.4), the conservation of mass equation is found:

$$
[\mathrm{H}]=\frac{1}{2}([\mathrm{DNA}]-[\mathrm{J}]),
$$

where [DNA] represents the total double-stranded DNA (dsDNA) concentration, which is constant and approximately equal to the sum of $[\mathrm{J}]_{0}$ and $2[\mathrm{H}]_{0}$. Solving for $[\mathrm{J}]$ in Eq. (2.5) and substituting into Eq. (2.3) gives a rate equation in terms of $[\mathrm{H}]$ :

$$
\frac{\mathrm{d}[\mathrm{H}]}{\mathrm{d} t}=k_{J \rightarrow H}([\mathrm{DNA}]-2[\mathrm{H}])^{2}-k_{H \rightarrow J}[\mathrm{H}],
$$

Integrating the rate equation ${ }^{83}$ and solving for $[\mathrm{H}]$ yields:

$$
[\mathrm{H}]=\frac{1}{2}[\mathrm{DNA}]-\frac{\sqrt{q}}{4 c}\left(\frac{1+\alpha e^{-\sqrt{q} t}}{1-\alpha e^{-\sqrt{q} t}}\right)+\frac{b}{4 c} .
$$

where the constants in Eq. (2.7) are given in terms of the rate constants and DNA concentration by:

$$
\begin{aligned}
& a=-\mathrm{k}_{H \rightarrow J}[\mathrm{DNA}], \\
& b=k_{H \rightarrow J}, \\
& c=2 k_{J \rightarrow H},
\end{aligned}
$$

and 


$$
\begin{aligned}
& q=-4 a c+b^{2}=8 k_{J \rightarrow H} k_{H \rightarrow J}[\mathrm{DNA}]+k_{H \rightarrow J}^{2}, \\
& \alpha=\frac{2 c[\mathrm{~J}]_{0}+b-\sqrt{q}}{2 c[\mathrm{~J}]_{0}+b+\sqrt{q}} .
\end{aligned}
$$

Details pertaining to the full derivation can be found in section 2.7.3.

\subsubsection{Fitting Reaction Kinetics Data}

To reduce the number of parameters used when fitting the reaction kinetics data, the rate equations were examined in the long time limit (i.e., $t=\infty$ ) at which the reaction equilibrium was reached. Thus, from Eq. (2.7), one obtains the following:

$$
[\mathrm{H}]_{t=\infty}=\frac{1}{2}[\mathrm{DNA}]-\frac{\sqrt{q}}{4 c}+\frac{b}{4 c} .
$$

Solving for $\frac{1}{2}[\mathrm{DNA}]$ in Eq. (2.11) and substituting into Eq. (2.7), the $\frac{b}{4 c}$ term is eliminated. Analyzing the newly simplified Eq. (2.7) for which $t=0$, one finds:

$$
[\mathrm{H}]_{t=0}=[\mathrm{H}]_{t=\infty}-\frac{\sqrt{q}}{2 c}\left(\frac{\alpha}{1-\alpha}\right) .
$$

Rearranging Eq. (2.12) to solve for the $\frac{\sqrt{q}}{2 c}$ term and substituting into Eq. (2.7), the $\frac{\sqrt{q}}{2 c}$ term is eliminated, thus yielding the following:

$$
[\mathrm{H}]=\left([\mathrm{H}]_{t=0}-[\mathrm{H}]_{t=\infty}\right)\left(\frac{(1-\alpha) e^{-\sqrt{q} t}}{1-\alpha e^{-\sqrt{q} t}}\right)+[\mathrm{H}]_{t=\infty}
$$

Because the $\alpha$ term is nearly zero (see Tables 2.8 and 2.9), Eq. (2.13) can be rewritten as:

$$
[\mathrm{H}]=\left([\mathrm{H}]_{t=0}-[\mathrm{H}]_{t=\infty}\right)\left(\frac{(1-\alpha) e^{-\sqrt{q} t}}{1-\alpha e^{-\sqrt{q} t}}\right)+[\mathrm{H}]_{t=\infty} .
$$

Thus, the fitting equation is equivalent to a decaying exponential function. To more accurately determine the rate constants, absorption spectra were obtained for completed reaction kinetics experiments performed at a variety of temperatures to ascertain the final concentrations of J-dimers and H-tetramers, thereby providing the equilibrium constant, $K_{e q}$, for the reaction at a series of temperatures. Expressing $\alpha$ in terms of $K_{e q}$ and 
substituting into Eq. (2.13) eliminates one of the unknown variables in the fit, such that only three parameters $\left(q,[\mathrm{H}]_{t=\infty}\right.$, and $\left.[\mathrm{H}]_{t=0}\right)$ are varied during the fitting procedure $(\mathrm{Eq}$. 2.14).

The temperature dependent rate constants appearing in Eq. (2.1) can be determined by evaluating reaction kinetics data obtained at different temperatures using Eq. (2.14). Assuming the reactions exhibit Arrhenius behavior, the activation energy and frequency factor for the forward $(\mathrm{J} \rightarrow \mathrm{H})$ and reverse $(\mathrm{H} \rightarrow \mathrm{J})$ reactions can then be calculated.

\subsubsection{Effects of DNA Concentration on J- and H-Aggregate Populations}

Because of the second-order nature of the proposed reaction mechanism, Eq. (2.1), the direction of the reaction will be dependent on the DNA concentration, with formation of the H-tetramers favored at high DNA concentration. Note that the effective dye concentration is proportional to the DNA concentration. Additionally, one can solve for a single population (i.e., [J] or [H]) by relating Eq. (5) to the equilibrium constant, $K_{e q}:$

$$
K_{e q}[\mathrm{~J}]^{2}=[\mathrm{H}]=\frac{1}{2}([\mathrm{DNA}]-[\mathrm{J}]),
$$

Rearranging Eq. (2.15) gives:

$$
2 K_{e q}[\mathrm{~J}]^{2}+[\mathrm{J}]-[\mathrm{DNA}]=0 .
$$

Solving for [J] and disregarding the negative solutions leads to:

$$
[\mathrm{J}]=\frac{\sqrt{1+8 K_{e q}[\mathrm{DNA}]}-1}{4 K_{e q}} .
$$

Similarly, $[\mathrm{H}]$ can be found to be: 


$$
[\mathrm{H}]=\frac{1}{2}\left([\mathrm{DNA}]-\frac{\sqrt{1+8 K_{e q}[\mathrm{DNA}]}-1}{4 K_{e q}}\right) .
$$

Thus, using Eqs. (2.17) and (2.18), while conserving mass, the following relationships expressing the relative fractions of $\mathrm{J}$-dimers and $\mathrm{H}$-tetramers in solution can be obtained:

$$
\begin{aligned}
& \frac{[\mathrm{J}]}{[\mathrm{J}]+2[\mathrm{H}]}=\frac{\sqrt{1+8 K_{e q}[\mathrm{DNA}]}-1}{4 K_{e q}[\mathrm{DNA}]}, \\
& \frac{2[\mathrm{H}]}{[\mathrm{J}]+2[\mathrm{H}]}=1-\frac{\sqrt{1+8 K_{e q}[\mathrm{DNA}]}-1}{4 K_{e q}[\mathrm{DNA}]},
\end{aligned}
$$

\subsection{Results and Discussion}

\subsubsection{Optical Analysis of Dye-Aggregate States}

To optically characterize each dye-aggregate state and identify key coherent exciton delocalization spectral signatures, the absorbance, fluorescence, and CD spectra were measured for Cy5-DNA systems in $1 \times \mathrm{TAE}$ buffer with $0 \mathrm{mM} \mathrm{MgCl} 2$ (J-dimer) and $155 \mathrm{mM} \mathrm{MgCl} 2$ (H-tetramer) added at a DNA concentration of $1.5 \mu \mathrm{M}$ (Figure 2.3). Spectral analysis of the dye monomer (Figure 2.3a) shows absorbance and emission peaks at 646 and $672 \mathrm{~nm}$, respectively, yielding a $26 \mathrm{~nm}$ Stokes shift, which is in accordance with the literature values. ${ }^{26,84}$ The full width at half-maximum (FWHM) of the absorbance (Abs) and fluorescence (FL) spectra were determined using a Gaussian fit to the data, as described further in section 2.7.5. As expected, the monomer did not produce an observable CD signal in the visible region of the spectrum (Figure 2.3b), indicating molecular achirality and the absence of excitonic coupling. In contrast, the right-handedness of the $\beta$-DNA macromolecule structure gives rise to an anticipated chiral induced CD signal in the UV region. ${ }^{85}$ Figure 2.3c, visualized using the UCSF Chimera software package, ${ }^{86,87}$ depicts a possible representation of the monomer Cy5 
internally functionalized onto a DNA duplex. The J-dimer optical spectra presented in Figure $2.3 \mathrm{~d}$ show similar spectral properties to those previously reported. ${ }^{26}$ The J-dimer absorbance spectrum showed a peak at $665 \mathrm{~nm}$ that is red-shifted nearly $20 \mathrm{~nm}$ relative to that of the monomer $\left(\lambda_{\max }=646 \mathrm{~nm}\right)$. Additionally, the J-dimer fluorescence maximum occurred at $667 \mathrm{~nm}$, corresponding to an almost imperceptible (2 nm) Stokes shift relative to the absorption maximum. Both the absorbance and fluorescence peaks underwent a narrowing of the FWHM by 20\% compared to the monomer. The narrowing of the Jdimer absorbance peaks can be attributed to exchange narrowing (i.e., motional narrowing), ${ }^{41}$ while the small Stokes shift and narrowing of the fluorescence peak are indicative of resonance fluorescence and consistent with J-aggregate behavior. ${ }^{22,46,47}$ Both exchange narrowing and resonance fluorescence are optical responses consistent with excitonic delocalization. To further corroborate the nature of the J-dimer, Figure 2.3e reveals a biphasic visible CD spectrum indicative of an EC-CD phenomenon, which is also characteristic of exciton delocalization. The observed +/- of the CD peaks, moving from long wavelength (i.e., low energy) to short wavelength (i.e., high energy), indicates right-handedness. ${ }^{48}$ The handedness of the J-dimer is most likely influenced by the righthanded $\beta$-DNA macromolecule. 

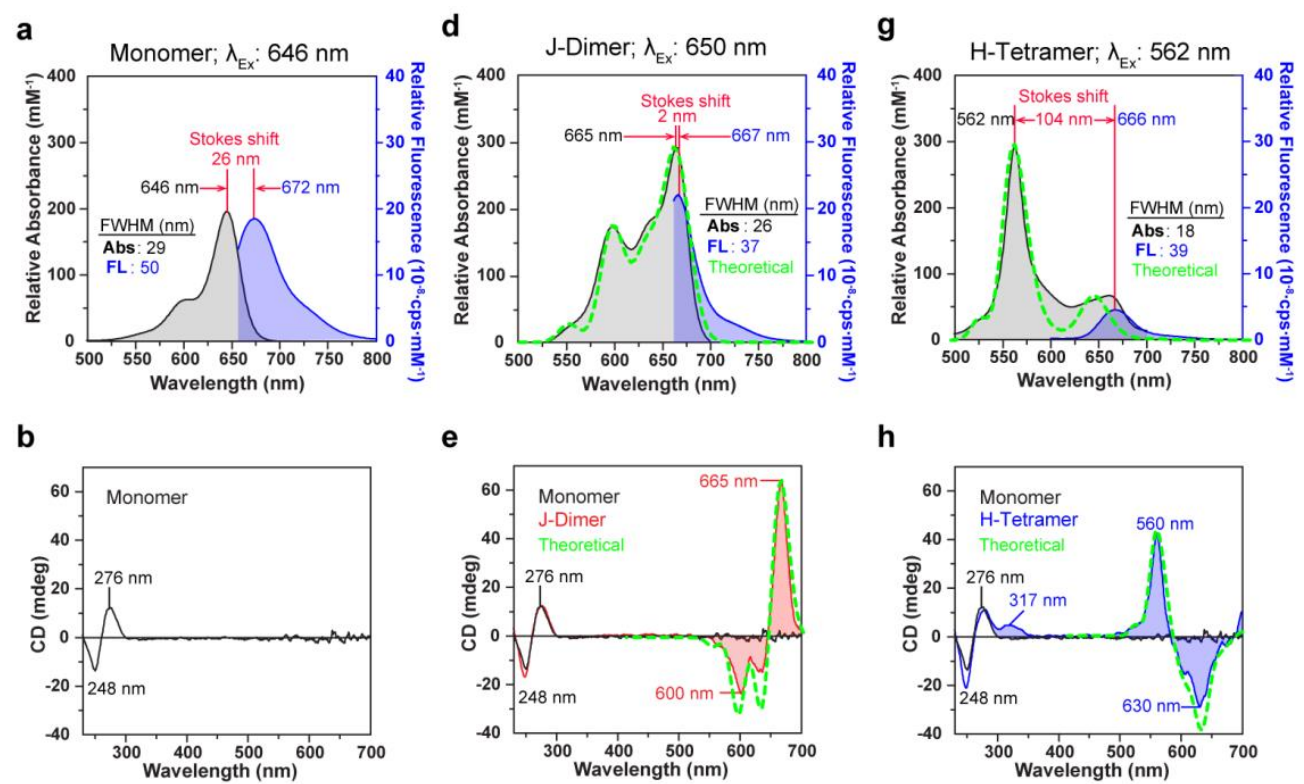

h

f
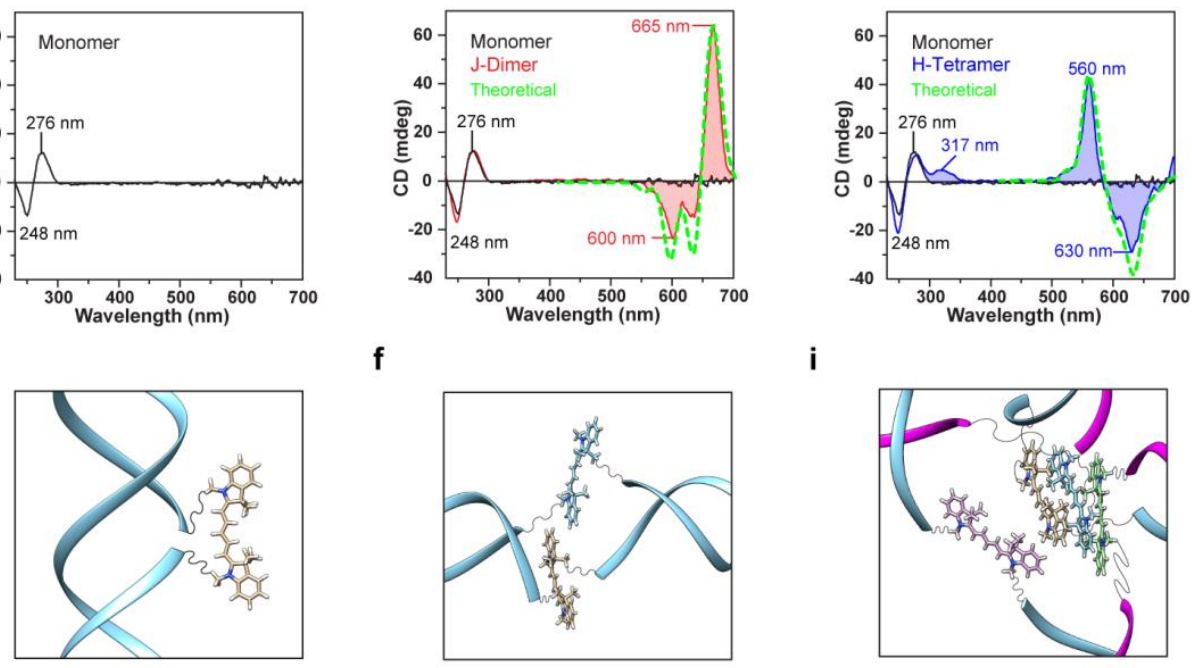

i

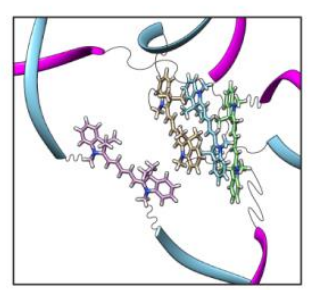

Figure 2.3. (a) Relative visible absorbance (black traces and ordinate axes), fluorescence emission (blue traces and ordinate axes), and (b) CD spectra of the monomer (15 $\mathrm{mM} \mathrm{MgCl}$ ). (c) Illustration depicting one possible representation of a single Cy5 dye internally functionalized onto a DNA duplex. (d) Relative visible absorbance, fluorescence emission, and (e) CD spectra for the J-dimer (duplex, $0 \mathrm{mM}$ $\mathrm{MgCl}_{2}$ ). (f) Illustrative representation of the J-dimer stacked in a head-to-tail arrangement within a DNA duplex. (g) Relative visible absorbance, fluorescence emission, and (h) CD spectra of the H-tetramer (155 $\left.\mathrm{mM} \mathrm{MgCl}_{2}\right)$ stacking arrangements. (i) Illustrative representation of the H-tetramer formed within a fourarmed DNA construct. Fluorescence spectra were acquired by exciting the dye constructs at their respective absorbance maxima. The FWHM of the absorbance (Abs) and fluorescence (FL) spectra were determined using a Gaussian fit, as described further in section 2.7.5. Theoretical fits (green dashed curves) were produced using in-house software as described further in section 2.7.2. All samples were prepared at $1.5 \mu \mathrm{M}$ DNA concentrations.

From fitting of the absorbance and $\mathrm{CD}$ data to the KRM model, the relative

distances and orientations between the Cy5 dyes can be predicted, thus providing the information necessary for visualizing the dye stacking arrangement of the J-dimer (Figure 2.3f). Fitting determined that the dyes are indeed stacked in a head-to-tail arrangement, as 
would be expected in a J-type aggregate, and oriented with a slight obliqueness that manifests itself in the optical spectra as an increase in the vibronic peak at $598 \mathrm{~nm}$. Additionally, it was determined that the center-to-center distance between the two dyes is $\sim 1.51 \mathrm{~nm}$, with the closest distance between the two dye molecules being $0.37 \mathrm{~nm}$. Fitting input parameters and output dye angles and positions are listed in Tables 2.4 and 2.5 , respectively. The H-aggregate (Figure $2.3 \mathrm{~g}$ ) exhibited a substantially blue-shifted absorbance peak at $562 \mathrm{~nm}$ as compared to the monomer $(646 \mathrm{~nm})$. Because of the semimobility of the $4 \mathrm{AJ}$, the $\mathrm{H}$-tetramer is in rapid equilibrium with each of the asymmetrical four-armed structures composed of two J-dimers (Figure 2.2). The simultaneous presence of both the blue-shifted (H-aggregate behavior) and red-shifted (J-aggregate behavior) peaks, with respect to the monomer peak, is hypothesized to result from the combination of the rapid equilibrium between the two aggregate states (i.e., H-tetramer and J-dimers) and an obliqueness in the dye stacking arrangement. Though the obliqueness of the dye stacking is akin to Davydov splitting, the aggregate exhibits predominantly $\mathrm{H}$-aggregate behavior. Here, the splitting is greater than $100 \mathrm{~nm}(\sim 336 \mathrm{meV})$, which is quite large, leading to an observed color change of the solution from cyan (monomer/J-dimer) to violet upon formation of H-tetramers. Additionally, with an emission peak maximum at $666 \mathrm{~nm}$, the system has a $104 \mathrm{~nm}$ Stokes shift relative to the blue-shifted absorbance peak. Similar to the J-dimer spectrum, the H-tetramer absorbance peak at $562 \mathrm{~nm}$ exhibited spectral narrowing (18 nm FWHM compared to the monomer peak's FWHM of $29 \mathrm{~nm}$ ) as a consequence of exchange narrowing. The fluorescence peak intensity, when excited at the H-tetramer absorbance maximum $(562 \mathrm{~nm})$, was reduced by a factor of 4 
relative to both the monomer and the J-dimer, as would be expected based on Kasha's molecular exciton theory for H-aggregates. ${ }^{14,15}$

Theoretical fitting of the H-tetramer absorbance and CD spectra (green dashed curves, Figure $2.3 \mathrm{~g}, \mathrm{~h}$ ) using the KRM model also provides insight into the orientation of the four dyes, which is schematically visualized in Figure 2.3i. The fitting input parameters and output dye angles and positions for the best fit are listed in Tables 2.4 and 2.5 , respectively. It was found that the dyes are positioned such that three dyes stack in a nearly parallel fashion with $\sim 0.4 \mathrm{~nm}$ separation, while the fourth dye stacks at an angle relative to the other dyes and with a somewhat greater nearest-neighbor separation. The variation in the orientation and spacing of the dyes relative to one another may occur due to strain within the $4 \mathrm{AJ}$ core. The best fit absorbance spectrum displays two peaks: one at $462 \mathrm{~nm}$, which matches the experimental absorbance peak, and the other at $650 \mathrm{~nm}$, which does not quite match the experimental red-shifted peak at $665 \mathrm{~nm}$ (Figure 2.3g). The obliqueness of this stacking arrangement enables a good fit of the KRM model to the observed EC-CD signal (Figure 2.3h). Interestingly, the CD spectrum reveals a -/+ biphasic signal, which is indicative of a left-handed excitonically coupled structure. The left-handedness of the dye stacking arrangement suggests that the dye aggregate is not influenced directly by the immediate DNA macromolecule structure, which is righthanded. Rather, the handedness most likely arises from the formation of the larger 4armed DNA construct. Curiously, a positive Cotton effect ${ }^{88}$ (i.e., right rotation of circularly polarized light) also appeared at $317 \mathrm{~nm}$, within the range of the DNA signal, for the H-tetramer. This may be related to interactions between the DNA and Cy5 dyes or result from the formation of the 4-armed structure. The shape of the experimental 
absorbance spectrum in the $650-700 \mathrm{~nm}$ range is similar to that of the dimer absorption spectrum, which suggests that the contribution at this end of the spectrum is due to internal rearrangements of the Holliday junction core in which dyes are paired as depicted in Figure 2.2. The experimental absorbance spectrum is a sum of the absorbance spectra of the individual core states. The discrepancy between the experimental and best fit absorbance spectra in this spectral region may thus be the result of attempting to find a single structure that best fits the spectrum produced by an ensemble of structures. A more rigorous test of the KRM model would require a reliable means by which to determine what the individual contribution is of each core state to the absorbance and CD spectra. 2.4.2 Investigation of Aggregation State Reaction Kinetics and Thermodynamics.

To enhance our understanding of the reaction by which $\mathrm{H}$-tetramer formation occurs, the kinetics and thermodynamics of the transition between J-dimer and $\mathrm{H}$ tetramer states were investigated. The procedure used to obtain reaction kinetics spectra as a function of time and temperature is illustrated in Figure 2.4a,b and described in detail in section 2.7.4. Reaction kinetics data (Figure 2.4c,d) were obtained by exploiting the Htetramer absorbance peak at $562 \mathrm{~nm}$ to monitor aggregate state changes with minimal spectral interference from the monomer absorbance centered at $645 \mathrm{~nm}$. Data were collected at several temperatures and fit to Eq. (14) to extract the rate constants. The average rate constants $\left(k_{J \rightarrow H}\right.$ and $\left.k_{H \rightarrow J}\right)$ at each temperature were computed and are listed in Tables 2.10 and 2.11. Figure 2.4c,d shows representative data sets (circles, squares, etc.) fit to Eq. (14) using the averaged rate constants (black curves). Our extracted rate constants were found to be relatively slow and on the order of $10^{-2}-10^{-4} \mathrm{~s}^{-1}$ and $10^{-5}$ $-10^{2} \mathrm{M}^{-1} \mathrm{~s}^{-1}$ for $k_{J \rightarrow H}$ and $k_{H \rightarrow J}$, respectively. Additionally, it was observed that 
increasing the temperature of the system by $10{ }^{\circ} \mathrm{C}$ led to a 2 orders of magnitude increase in the reaction rate constants, which exhibited Arrhenius-type behavior. The slow rate of the reaction would be expected for a case in which two DNA duplexes undergo four-way branch migration to form a 4AJ. Specifically, the magnesium ions present in solution, as opposed to monovalent sodium ions, have been shown to slow the rate of reaction. ${ }^{77,89}$
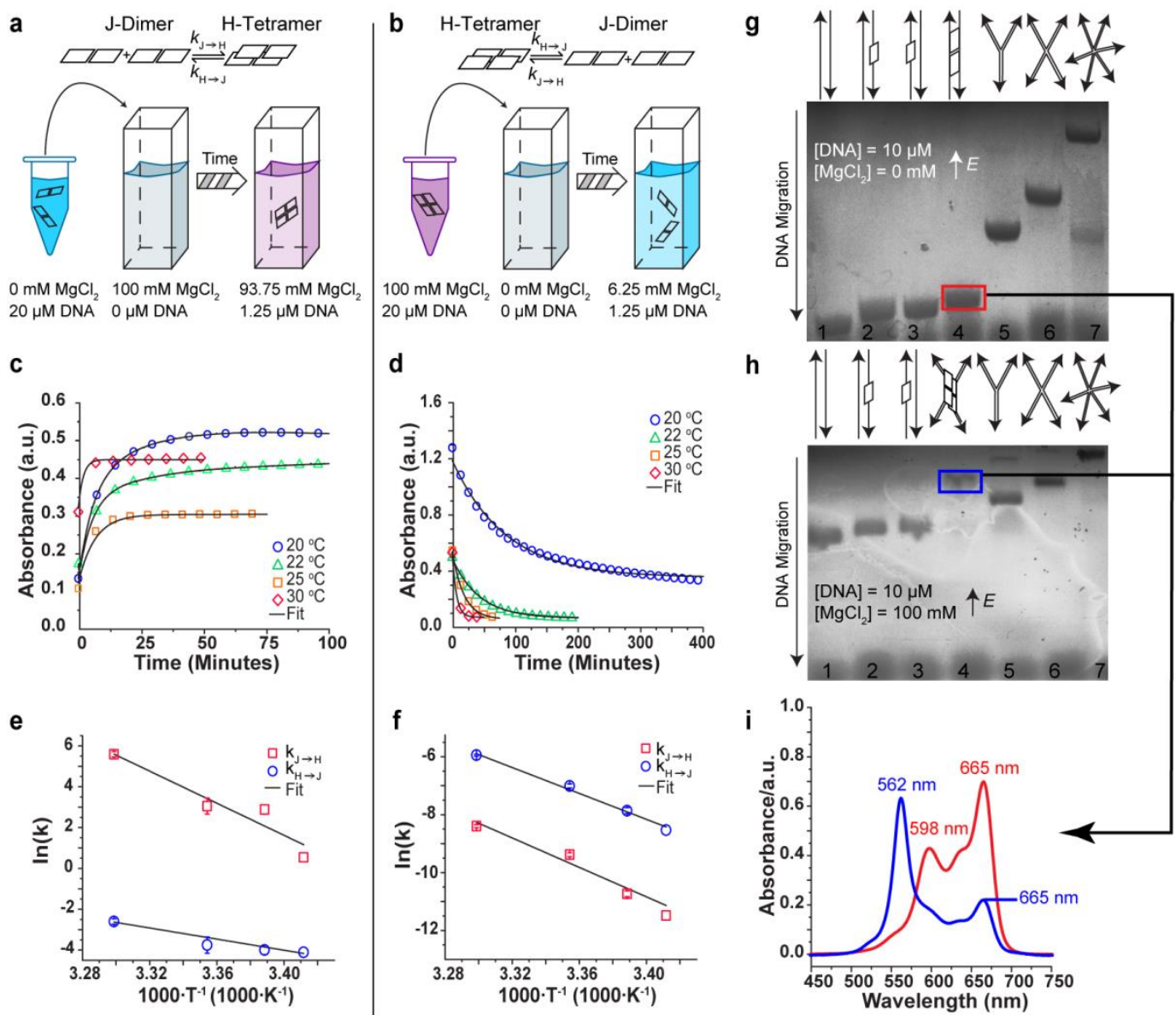

Figure 2.4. Schematic illustrations of the reaction kinetics setup for solutions transitioning from (a) duplexes (J-dimers, 0 mM MgCl ) to 4AJs (H-tetramers, 93.75 $\mathrm{mM} \mathrm{MgCl}_{2}$ ) and (b) 4AJs (H-tetramers, $100 \mathrm{mM} \mathrm{MgCl}$ ) to duplexes (J-dimers, 6.25 mM MgCl2). Initial dye-DNA solutions were prepared at DNA concentrations of 20 $\mu M$ to yield final concentrations of $1.25 \mu M$ after dilution. Colors shown represent actual experimentally observed color changes. The depicted dye stacking arrangements are schematic representations and are not meant to imply specific geometric configurations. Corresponding reaction kinetics data of the timedependent transitions: (c) $\mathrm{J}_{0} \rightarrow \mathrm{H}_{93.75}\left(\mathrm{O} \mathrm{mM} \rightarrow 93.75 \mathrm{mM} \mathrm{MgCl}\right.$ ) and (d) $\mathrm{H}_{100} \rightarrow \mathrm{J}_{6.25}$ $(100 \rightarrow 6.25 \mathrm{mM} \mathrm{MgCl}$ ). Shown are representative data sets fit to Eq. (14) using the 
averaged rate constant measured over multiple runs for a given temperature. The log of the averaged forward $\left(k_{J \rightarrow H}\right)$ and reverse $\left(k_{H \rightarrow J}\right)$ rate constants obtained from a total of three independent measurements are plotted versus the inverse temperature for (e) $\mathrm{J}_{0} \rightarrow \mathrm{H}_{93.75}$ and (f) $\mathrm{H}_{100} \rightarrow \mathrm{J}_{6.25}$, respectively, to determine the activation energies of the forward $\left(E_{J \rightarrow H}\right)$ and reverse $\left(E_{H \rightarrow J}\right)$ reactions based on the best fit to the data (black line). Error bars were determined using the standard deviation. Structural characterization of the aggregates using electrophoresis to monitor the migration of (g) $\mathrm{J}$ - and (h) $\mathrm{H}$-aggregates, prepared at $\mathrm{O}$ and $100 \mathrm{mM}$ added $\mathrm{MgCl}_{2}$, respectively, relative to a variety of DNA constructs (schematically represented). Absorbance spectra (i) of the excised and eluted bands were obtained for the J-dimer (red box) and the H-tetramer (blue box).

Table 2.1. Activation energies $\left(E_{J \rightarrow H}\right.$ and $\left.E_{H \rightarrow J}\right)$, frequency factors $(A)$, and free energy $\left(\Delta G^{\circ}\right)$ values for aggregation state transitions.

\begin{tabular}{|c|c|c|c|c|c|}
\hline State & $\begin{array}{c}\boldsymbol{E}_{\boldsymbol{J} \rightarrow \boldsymbol{H}} \\
(\mathrm{kJ} / \mathrm{mol})\end{array}$ & $\begin{array}{c}\boldsymbol{E}_{\boldsymbol{H} \rightarrow \boldsymbol{J}} \\
(\mathrm{kJ} / \mathrm{mol})\end{array}$ & $\begin{array}{c}\boldsymbol{A}_{\boldsymbol{J} \rightarrow \boldsymbol{H}} \\
\left(\mathrm{M}^{-1} \mathrm{~s}^{-1}\right)\end{array}$ & $\begin{array}{c}\boldsymbol{A}_{\boldsymbol{H} \rightarrow \boldsymbol{J}} \\
\left(\mathrm{s}^{-1}\right)\end{array}$ & $\begin{array}{c}\Delta \boldsymbol{G}^{\circ} \\
(\mathrm{kJ} / \mathrm{mol})\end{array}$ \\
\hline $\mathrm{J}_{0}-\mathrm{H}_{93.75}$ & $\begin{array}{c}328.3 \pm 7.3 \\
(\sim 3.4 \mathrm{eV})\end{array}$ & $\begin{array}{c}211.6 \pm 46.6 \\
(\sim 2.2 \mathrm{eV})\end{array}$ & $(2.3 \pm 6.8) \times 10^{59}$ & $(6.3 \pm 1.2) \times 10^{32}$ & $\begin{array}{c}-22.5 \\
(-0.23 \mathrm{eV})\end{array}$ \\
\hline $\mathrm{H}_{100}-\mathrm{J}_{6.25}$ & $\begin{array}{c}113.8 \pm 6.1 \\
(\sim 1.2 \mathrm{eV})\end{array}$ & $\begin{array}{c}186.2 \pm 41.8 \\
(\sim 1.9 \mathrm{eV})\end{array}$ & $(2.5 \pm 7.3) \times 10^{18}$ & $(3.5 \pm 5.9) \times 10^{28}$ & $\begin{array}{c}7.1 \\
(0.07 \mathrm{eV})\end{array}$ \\
\hline
\end{tabular}

Plotting the log of the averaged rate constants against the inverse of temperature, the activation energies (listed in Table 2.1) for the aggregation state transitions were calculated using a linear fit (Figure 2.4e,f). The reported values for activation energies of the dimerization of free-floating dyes are typically a factor of 4 smaller than what we report in Table 2.1. However, this composite two-state system consists of both dyes and DNA oligomers and thus is expected to exhibit strong temperature dependence due to physical processes such as DNA breathing and macromolecular rearrangement. A deeper understanding of the activation energy and frequency factor values will require structural and molecular dynamic information on the process leading to the transformation of a pair of J-dimers into an H-tetramer. 
To calculate the free energy of the system, the Gibbs Free energy $\left(\Delta \mathrm{G}^{\circ}\right)$ was computed as described in section 2.7.7. The $\mathrm{J}_{0} \rightarrow \mathrm{H}_{93.75}$ reaction, in which two J-dimers (duplexes) combine to form an $\mathrm{H}$-tetramer (4AJ), has a $\Delta \mathrm{G}^{\circ}$ of $-22.5 \mathrm{~kJ} / \mathrm{mol}$, while the $\mathrm{H}_{100} \rightarrow \mathrm{J}_{6.25}$ reaction, in which an H-tetramer dissociates into two J-dimers, has a $\Delta \mathrm{G}^{\circ}$ value of $+7.1 \mathrm{~kJ} / \mathrm{mol}$ (Table 2.1). Because the system's entropy decreases with the formation of an $\mathrm{H}$-tetramer and increases with the dissociation of $\mathrm{H}$-tetramers into $\mathrm{J}$ dimers, we can extrapolate that the enthalpy of the system must be negative for $\mathrm{J}_{0} \rightarrow$ $\mathrm{H}_{93.75}$ transitions and positive for $\mathrm{H}_{100} \rightarrow \mathrm{J}_{6.25}$ transitions in the temperature range studied. The relatively small $\Delta \mathrm{G}^{\circ}$ values are consistent with our hypothesis of $4 \mathrm{AJ}$ formation due to similar base-pairing energy of the two states. Thus, the differences in the free energy arise primarily from dye-dye interactions and the change in configurational entropy. ${ }^{77}$

On the basis of the outcome of the spectral fitting using the KRM model (Figure 2.3) and the success of the second-order reaction kinetics model in fitting the reaction kinetics data (Figure 2.4), it was hypothesized that the H-tetramer consisted of a complex having four duplex arms extending from the central core, and thus should exhibit mobility, under nondenaturing gel electrophoresis conditions, comparable to that of a four-armed Holliday junction with arms of the same length (Figure 2.4g,h). These expectations were confirmed by gel shift experiments in which the mobility of the $\mathrm{H}$ tetramer was compared with that of a Holliday junction and an assortment of controls. Details of the experiments and multiarmed DNA structure designs are provided in section 2.7.6. The results show ( $0 \mathrm{mM} \mathrm{MgCl}_{2}$, Figure $\left.2.4 \mathrm{~g}\right)$ that the J-dimer (lane 4) migrates through the gel at a mobility rate similar to that of the duplex structures (lanes 1-3), indicating that a duplex persists without higher-ordered aggregation. As expected for high 
salt concentration (100 $\mathrm{mM} \mathrm{MgCl}_{2}$, Figure 2.4h), the transport of the H-tetramer (lane 4) mimics the mobility of a four-armed structure (lane 6) - as opposed to smaller structures such as the duplexes (lanes 1-3) and three-armed construct (lane 5) or a larger structure such as the six-armed construct in lane 7. This structural information supports our hypothesis that the H-tetramer consists of two DNA duplexes combined to form a fourarmed construct, stabilized by the four centrally located Cy5 dyes. The identities of the Jdimer (red box and corresponding absorbance curve) and H-tetramer bands (blue box and corresponding absorbance curve) were confirmed by excising the bands, eluting their contents, and measuring their respective absorbance curves (Figure 2.4i). Overall, it was found that the proposed reaction kinetics model [Eq. (1)] described the experimental reaction kinetics data extremely well (Figure $2.4 \mathrm{c}, \mathrm{d}$ ). An implication of the goodness of the fits of the data to a two-state second-order reaction kinetics model [Eq. (1)] is that the size (i.e., order) of the dye aggregation is explicitly 2 -fold and that higher-ordered aggregation (i.e., formation of multimers beyond $\mathrm{H}$-tetramers) is not observed. Thus, unlike free-floating dyes that form large aggregates, ${ }^{91-93}$ our dye-DNA system only forms small $\mathrm{H}$-tetramer aggregates. The formation of $\mathrm{H}$-tetramers, as opposed to larger aggregates such as $\mathrm{H}$-hexamers, is further substantiated by the PAGE characterization (Figure $2.4 \mathrm{~g}, \mathrm{~h}$ ), which explicitly correlates the mobility of the H-tetramer construct to that of a four-armed structure.

\subsubsection{Effects of Salt and DNA Concentrations on J- and H-Aggregate Populations.}

Precise arrangement of dye aggregates is essential for controlling excitonic delocalization behavior. Because it has been shown that both dye and salt concentration affect the aggregation of free-floating dyes,${ }^{49-53,91,92,94,95}$ we explored the effects of both 
salt and DNA concentrations on the preferred Cy5 dye stacking arrangements within our DNA-templated system to test the proposed reaction model [Eq. (1)]. Note that the DNA concentration is proportionate to the dye concentration and the salt concentration is explicitly added salt to a TAE buffer solution. After collecting the UV-vis absorbance spectrum of each sample (Figure 2.5a,b), the spectra of PAGE purified controls (section 2.7.8) were used to calculate the relative percentage of $\mathrm{J}$ - and $\mathrm{H}$-aggregate populations present in solution at various salt $\left(0-100 \mathrm{mM}\right.$ added $\left.\mathrm{MgCl}_{2}\right)$ and DNA $(0.1-10 \mu \mathrm{M})$ concentrations. Figure 2.5a,b shows that with changes in salt or DNA concentration the locations of the absorbance peaks remain constant within the selected $\mathrm{MgCl}_{2}$ and $\mathrm{DNA}$ concentration ranges, respectively. The absence of variation in the position of the absorption peaks implies that the relative orientations between the two dyes are constant for the two populations, suggesting that the dyes do not continuously rearrange their configuration as a function of salt concentration or DNA concentration but instead exist in one of two geometrically distinct states, as a J-dimer (duplex) or an H-tetramer (4AJ). Accordingly, the relative concentration of each aggregate state varies with salt concentration and/or DNA concentration as indicated by the change in relative peak intensities for the J-dimer $\left(\lambda_{\max }=665 \mathrm{~nm}\right)$ and the H-tetramer $\left(\lambda_{\max }=562 \mathrm{~nm}\right)$. Furthermore, as shown in Figure 2.17, absorption spectra at intermediate salt or DNA concentrations are well approximated using a linear combination of the PAGE purified Jand H-aggregate spectra. Thus, the observed spectra are a manifestation of spectral overlap between $\mathrm{J}$ - and H-aggregates. 
a
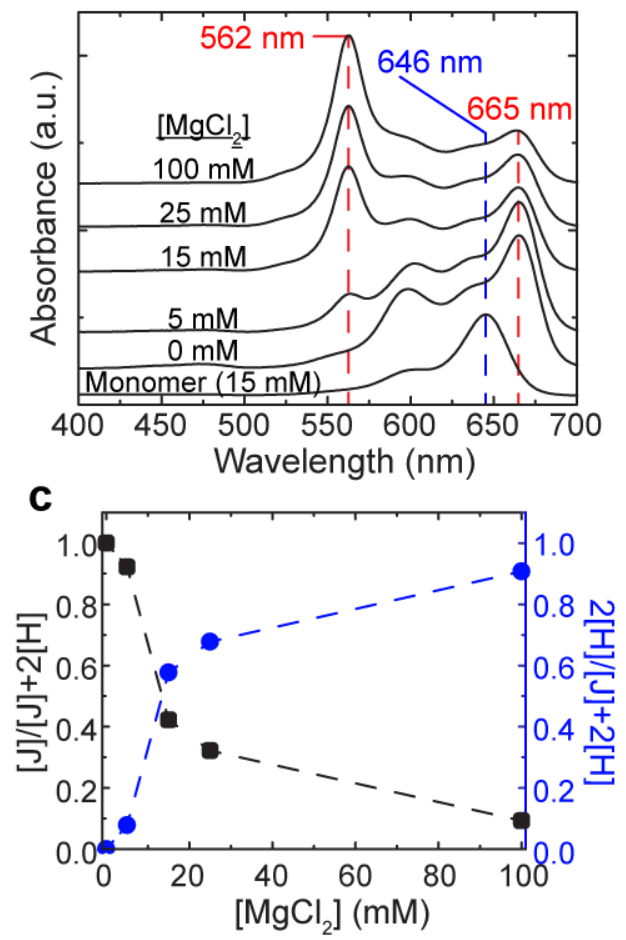

b

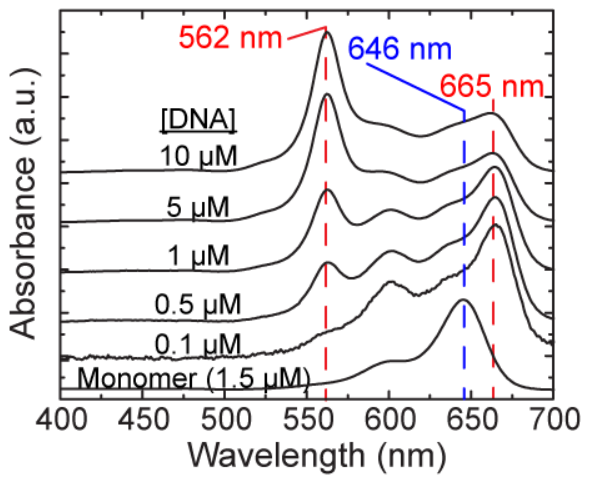

d

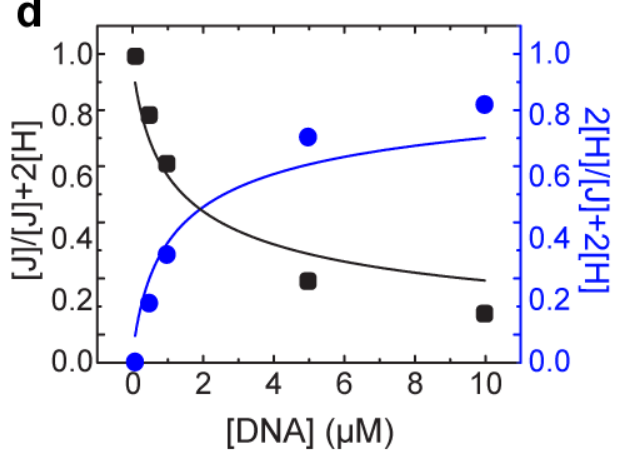

Figure 2.5. Observed UV-vis absorbance spectra with varied (a) salt (DNA concentration held constant at $1.5 \mu \mathrm{M}$ ) and (b) DNA (added $\mathrm{MgCl}_{2}$ concentration held constant at $15 \mathrm{mM}$ ) concentrations. Changes in J-dimer (black squares and ordinate axes) and H-tetramer (blue circles and ordinate axes) fractions (based on their respective absorbance peaks) as a function of (c) salt (i.e., $\mathrm{MgCl}_{2}$ ) and (d) DNA concentrations. The dashed lines connecting the points in panel $\mathrm{c}$ are for visual aid only, while the curves in panel $d$ were obtained by fitting the data according to Eqs (19) and (20). Each spectrum was acquired at room temperature.

Using the fitting procedure described in section 2.7.8, the relative populations of $\mathrm{J}$ - and $\mathrm{H}$ - aggregates can be determined. Figure $2.5 \mathrm{c}$ shows the changes in aggregate populations (taking into account conservation of mass) as a function of salt (i.e., added $\mathrm{MgCl}_{2}$ ) concentration. Plotting the changes in ratio of $\mathrm{J}$ - to $\mathrm{H}$-aggregates as a function of salt concentration revealed a sigmoidal-like trend in which higher $\mathrm{MgCl}_{2}$ concentrations produced a favorable environment for $\mathrm{H}$-tetramer formation (Figure 2.5c). The dye studied, $\mathrm{Cy} 5$, has been found to naturally arrange into $\mathrm{H}$-aggregates at high salt and dye concentrations, ${ }^{96}$ and recent studies from Mooi et al. show that the addition of ionic species can promote higher-ordered aggregation of cyanine-based dyes. ${ }^{91,92}$ Furthermore, 
it is also known that an excess of $\mathrm{Mg}^{2+}$ can aid with neutralizing the negative charge of the DNA backbone, thereby decreasing the charge repulsion between adjacent DNA duplexes and allowing two J-dimers to interact within proximal distances. Thus, it is not surprising that the dye-DNA constructs tend to assemble into H-tetramers at these high ionic strengths. Figure $2.5 \mathrm{~d}$ displays how the aggregate population composition changes with increasing DNA concentration. If the transformation between $\mathrm{J}$ - and H-aggregates were simply first order (as expected for dye conformational changes within a DNA duplex), changes would not be observed in the relative concentrations of J-dimers ([J]) to H-tetramers $([\mathrm{H}])$ as a function of the DNA concentration, [DNA]. Furthermore, the percentages of J-dimers and H-tetramers present in solution, defined as $[\mathrm{J}] /([\mathrm{J}]+2[\mathrm{H}])$ and $2[\mathrm{H}] /([\mathrm{J}]+2[\mathrm{H}])$, would be constants independent of [DNA]. The dependence of the relative populations of $\mathrm{J}$ - and $\mathrm{H}$-aggregates on [DNA] provides a strong indication that a second-order reaction mechanism is involved, and agrees with previous studies that show aggregation is concentration dependent. ${ }^{51-53,93,96,97}$ As shown by the fit line in Figure 2.5d, the observed trend correlates well with Eqs. (19) and (20), lending support for the proposed second-order reaction described by Eq. (1) and used to fit the data as demonstrated in Figure 2.4.

\subsubsection{Distance Dependence of Coherent Exciton Delocalization.}

Exciton delocalization results from strong excitonic coupling between neighboring dyes located with a spatial proximity of less than a few nanometers. ${ }^{59}$ Because the dimensions of the DNA helix are well-known, varying the number of bps separating the Cy5 dye pair within the DNA construct provides a means for examining the dependence of the dye coupling strength on spatial separation (Figure 2.6). 


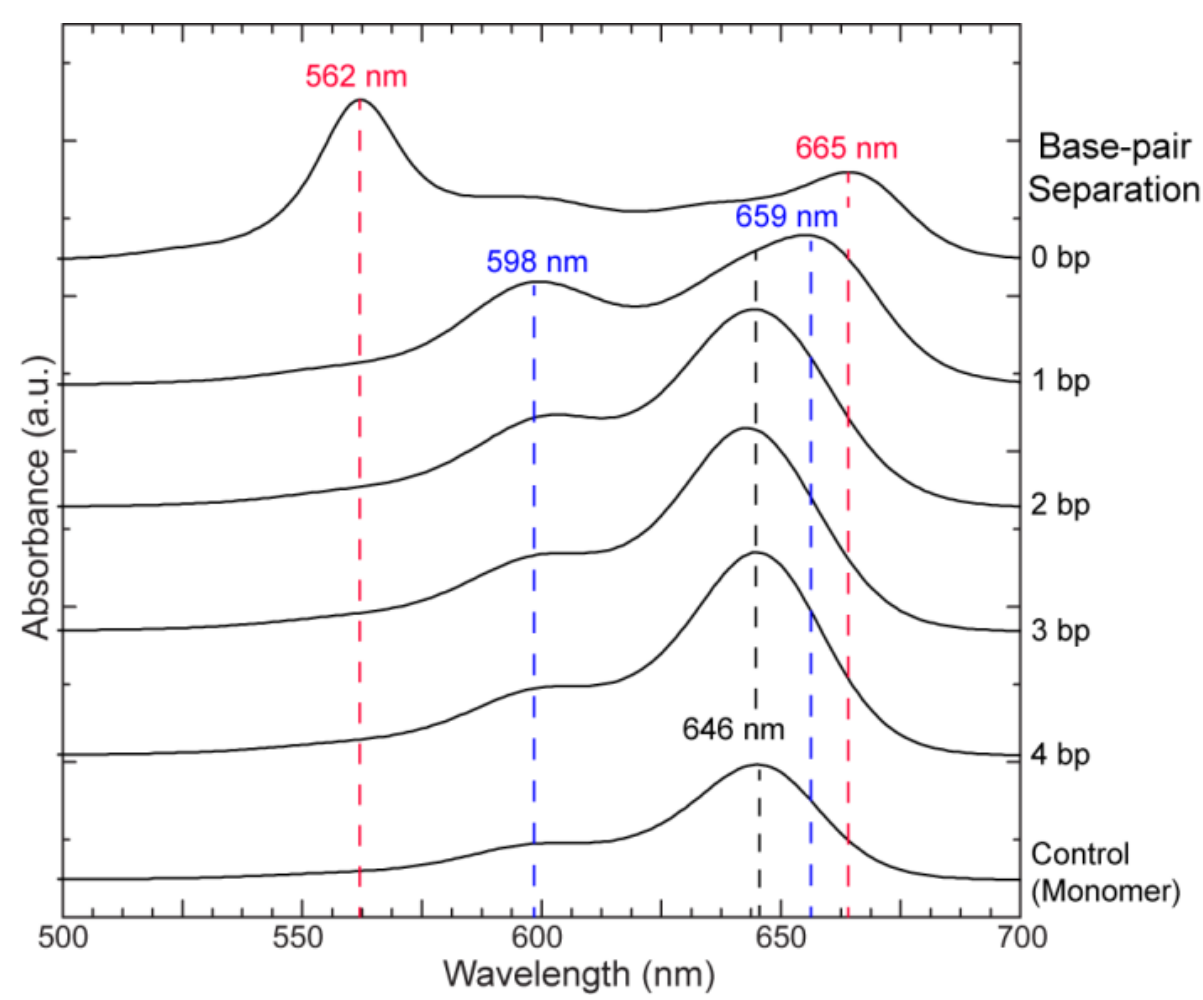

Figure 2.6 UV-Vis absorbance spectra documenting changes in the optical properties of dye aggregates as the number of bps separating the dye pair is increased. Samples were prepared at $1.0 \mu \mathrm{M}$ DNA concentration with $15 \mathrm{mM} \mathrm{MgCl} 2$ added. Spectra are normalized by DNA concentration.

In Figure 2.6, the absorbance spectra of the variable bp-separated dye-pairs are compared to the monomer (i.e., control), which shows a single peak at $646 \mathrm{~nm}$ with a small vibronic shoulder at $599 \mathrm{~nm}$. At high salt concentration and $0 \mathrm{bp}$ separation, the monomer peak at $646 \mathrm{~nm}$ is absent, while both a blue-shifted peak at $562 \mathrm{~nm}$ (characteristic of an H-aggregate) and a red-shifted peak at $665 \mathrm{~nm}$ (characteristic of Jaggregate behavior) appear simultaneously, consistent with Davydov splitting. The large Davydov splitting $(103 \mathrm{~nm})$ results in an observable color change of the solution from cyan (monomer) to violet, which is a visible indication of coherent exciton delocalization. Upon increasing the dye pair separation to $1 \mathrm{bp}$, the $\mathrm{H}$-aggregate peak completely disappears, while the $\mathrm{J}$-aggregate peak is only shifted slightly to $659 \mathrm{~nm}$ (i.e., $13 \mathrm{~nm}$ relative to the monomer rather than $19 \mathrm{~nm}$ as in the case of 0 bp separation), suggesting 
that $\mathrm{H}$-tetramer formation is no longer favorable, and the coupling between the $\mathrm{J}$-dimer dyes has weakened. The increase in the vibronic peak at $598 \mathrm{~nm}$ correlates well to the vibronic effects discussed by Spano with the formation of J-aggregates. ${ }^{98}$ Increasing the distance to 2 bp separation returns the spectrum to that of the monomeric form, implying that coupling of the dyes has ceased. While these data test the variability of spatial separation along with dye orientation (i.e., resulting from the helical nature of the DNA template) and the local chemical environment (i.e., due to differences in neighboring base pairs), overall we find that the absorption spectrum profile of the dye aggregates with $2-4$ bp separation reflect the shape of the monomer spectrum, indicating that spatial proximity has the greatest effect on the Davydov splitting. Moreover, the extremely large band splitting at relatively small $(<2 \mathrm{~nm})$ distances, in combination with both $\mathrm{J}$ - and $\mathrm{H}-$ aggregate optical behavior, corresponds well with models described by Kasha ${ }^{14,15}$ and provides strong evidence for coherent exciton delocalization.

\subsection{Conclusion}

In this study, we have investigated excitonic delocalization in a DNA-templated dye aggregate system. Specifically, it was found that a Cy5 dye-DNA dimer construct, which exhibits primarily J-aggregate characteristics at low salt and DNA concentrations, can couple with a second dimer to form a tetramer with predominantly $\mathrm{H}$-aggregate characteristics at higher salt and/or DNA concentrations. Characterizing the reaction kinetics and subsequent thermodynamic equilibrium behavior of this two-state system combined with performing gel electrophoresis studies suggested that the H-tetramer formed through a branch migration process in which a four-armed DNA construct was assembled with four Cy5 dyes located centrally. The oblique stacking of these 
arrangements, as evidenced by EC-CD signals and validated by fitting the spectral data with the KRM model, results in a large Davydov splitting of the absorbance spectrum as well as a significant change of the solution color from cyan to violet. Furthermore, we show that the relative concentration of the two aggregate states (i.e., J-dimer versus Htetramer) can be controlled by changing the salt and/or DNA concentrations, such that $\mathrm{H}$ tetramer aggregates are favored at high salt and/or DNA concentrations. The large deviation we have observed in the absorption spectra of the J-dimer and $\mathrm{H}$-tetramer configurations compared to that of the monomer indicates that further investigation of the use of DNA-templated dye aggregates with controlled structure is warranted. The development of precisely assembled dyes that exhibit exciton delocalization may enable the construction of aggregates in configurations that are not easily accessible in simple dye-solvent systems. The delocalization of excitons over such dye aggregates may allow the tailoring of absorption and fluorescence spectra for application in light harvesting, analysis of chemicals, and biological imaging. Advancing the development of DNAtemplated dye aggregate systems even further by assembling complex networks of dyes to propagate exciton signals via coherent exciton exchange may enable information processing and perhaps quantum computing.

\subsection{Acknowledgments}

This research was supported in part by the Boise State University Division of Research and Economic Development, NASA Idaho Space Grant Consortium (ISGC), the National Science Foundation INSPIRE No. 1648655, the Institutional Development Awards (IDeA) from the National Institute of General Medical Sciences No. P20GM103408 and the National Institutes of Health: No. P20GM109095, The National 
Science Foundation No 0619793 and 0923535, the MJ Murdock Charitable Trust, and the Idaho State Board of Education. B.L.C. acknowledges the support of the Micron Technology MSE Ph.D. Fellowship. The authors thank Don and Jan Haworth for financial assistance, the students and staff within the Nanoscale Materials \& Device group for valuable assistance with this work, Raquel Brown (Boise State University) for aid with CD data collection, Hieu Bui (Duke University) for conducting preliminary work, Jennifer Padilla (J. E. Padilla studio) for insightful discussions on graphics, and Dr. Lan Li, Eric Nelson, and Andres Correa for DFT modeling. Additionally, we appreciate the helpful discussion with Dr. Joseph Melinger and colleagues at the U.S. Naval Research Laboratory (NRL) regarding the theoretical work of Kühn et al. Molecular graphics and analyses were performed with the UCSF Chimera package. Chimera is developed by the Resource for Biocomputing, Visualization, and Informatics at the University of California, San Francisco (supported by NIGMS P41GM103311).

\subsection{Supporting Information}

\subsubsection{Strand Sequences and Dye Details}

The sequences for both the dye labeled (i.e., Cy5) and non-dye labeled (i.e., ND) DNA oligomers are given in Table 2.2 below. Oligonucleotides were purchased from Bio-Synthesis, Inc. (Lewisville, TX, USA) and Integrated DNA Technologies, Inc. (Coralville, IA, USA). Comparable results were obtained with strands purchased from either manufacturer. Strands labeled Cy5-0 through Cy5-4 were used for distance dependence studies that examined the effects of base-pair (bp) separation of dye pairs on exciton delocalization. The Cy5 structure is illustrated in Figure 2.7, and shows the attachment sites by which the dye is covalently bound to the 5' and 3' sides of the DNA 
backbone, respectively. Optical properties of the dye monomer, J-dimer, and $\mathrm{H}$-tetramer are listed in Table 2.2. It should be noted that the extinction coefficients for the aggregates are expected to change due to the difference in the number of dyes (e.g., one for the monomer versus four for the tetramer) and to account for exciton-vibrational interactions. The large three, four, and six-armed structures are illustrated in Figure 2.8. 
Table 2.2 DNA Oligomer Sequences

\begin{tabular}{|c|c|c|c|}
\hline $\begin{array}{l}\text { Strand } \\
\text { Name }\end{array}$ & Sequence $\left(5^{\prime}\right.$, to $\left.3^{\prime}\right)$ & $\begin{array}{c}\text { Length } \\
\text { (nt) }\end{array}$ & Purification \\
\hline ND & CAGTCATAATATGCGAGCGATTATAT & 26 & Standard Desalting* \\
\hline Сy5-0 & CAGTCATAATATGCGA/Cy5/GCGATTATAT & 26 & HPLC $* *$ \\
\hline $\mathrm{cND}$ & ATATAATCGCTCGCATATTATGACTG & 26 & Standard Desalting \\
\hline cСy5-0 & ATATAATCGC/Cy5/TCGCATATTATGACTG & 26 & HPLC \\
\hline Cy5-1 & ATATAATCGCT/Cy5/CGCATATTATGACTG & 26 & HPLC \\
\hline Сy5-2 & ATATAATCGCTC/Cy5/GCATATTATGACTG & 26 & HPLC \\
\hline Cy5-3 & ATATAATCGCTCG/Cy5/CATATTATGACTG & 26 & HPLC \\
\hline Cy5-4 & ATATAATCGCTCGC/Cy5/ATATTATGACTG & 26 & HPLC \\
\hline $3 \mathrm{~A}$ & ATATAATCGCTCGCATATTATGACTG & 26 & Standard Desalting \\
\hline $3 \mathrm{~B}$ & CAGTCATAATATGTGGAATGTGAGTG & 26 & Standard Desalting \\
\hline $3 \mathrm{C}$ & CACTCACATTCCACGAGCGATTATAT & 26 & Standard Desalting \\
\hline $4 \mathrm{C}$ & САСТCACАТТССАСТСААСАССАСАА & 26 & Standard Desalting \\
\hline $4 \mathrm{D}$ & TTGTGGTGTTGAGCGAGCGATTATAT & 26 & Standard Desalting \\
\hline $6 \mathrm{~A}$ & CAAGGTATCATCGGTTCAAGTCCAGT & 26 & Standard Desalting \\
\hline $6 \mathrm{~B}$ & ACTGGACTTGAACGCTAACTATCTGG & 26 & Standard Desalting \\
\hline $6 \mathrm{C}$ & CCAGATAGTTAGCCCGAGAGCATTTT & 26 & Standard Desalting \\
\hline $6 \mathrm{D}$ & AAAATGCTCTCGGTGTCCCGTGTAAA & 26 & Standard Desalting \\
\hline $6 \mathrm{E}$ & TTTACACGGGACATTATTCAGGGCAC & 26 & Standard Desalting \\
\hline $6 \mathrm{~F}$ & GTGCCCTGAATAACGATGATACCTTG & 26 & Standard Desalting \\
\hline
\end{tabular}

* Desalting to remove short products and small organic contaminants. Does include PAGE purification

** High-Performance Liquid Chromatography 
The common name, formal name, and structure of the dye used in this study is:

Cy5: 1-[3-(4-monomethoxytrityloxy)propyl]-1'-[3-[(2-cyanoethyl)-(N,N-diisopropyl

phosphoramidityl]propyl]-3,3,3',3'-tetramethylindodicarbocyanine chloride.

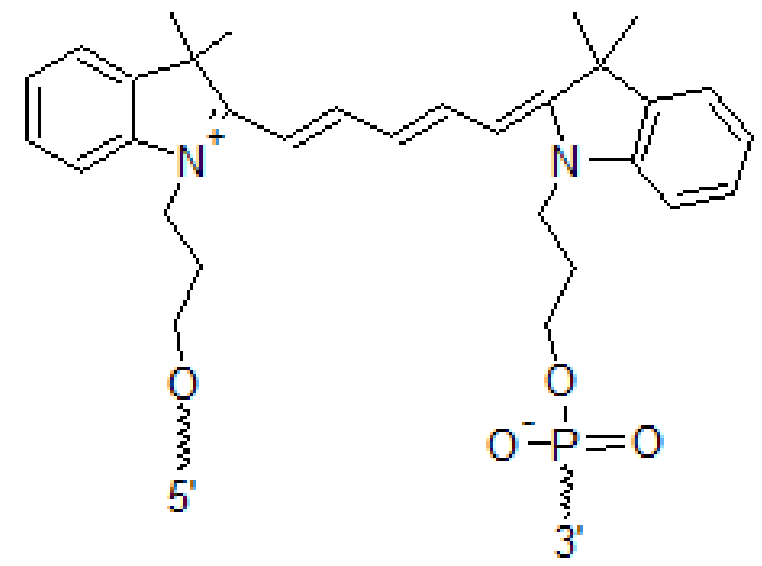

Figure 2.7. Molecular structure of the Cy5 dye adapted from IDT. The two long carbon tethers are used to attach onto the DNA oligomers. The attachment sites by which the dye is covalently bound to the DNA backbone are indicated by 5' and 3', representing the directionality of the attachment scheme.

Table 2.3 List of Absorbance Peak Maxima, Extinction Coefficients, and Full Widths at Half Maximum (FWHM) for a variety of dye-DNA constructs.

\begin{tabular}{|c|c|c|c|}
\hline $\begin{array}{c}\text { Dye-DNA } \\
\text { Construct }\end{array}$ & $\begin{array}{c}\text { Absorbance Peak } \\
\text { Maximum (nm) }\end{array}$ & $\begin{array}{c}\text { Extinction } \\
\text { Coefficient at Max } \\
\left(\mathbf{L ~} \mathbf{~ m o l}^{\mathbf{- 1}} \mathbf{c m}^{\mathbf{- 1}}\right)\end{array}$ & FWHM (nm) \\
\hline Cy5 Monomer & 646 & 250,000 & 29 \\
\hline J-Dimer & 665 & 376,220 & 26 \\
\hline H-Tetramer & 562 & 398,800 & 18 \\
\hline
\end{tabular}




\subsubsection{Fitting of the Absorption and Circular Dichroism (CD) Data using the Kühn-}

\section{$\underline{\text { Renger-May Model }}$}

There is a substantial collection of work that focuses on the modeling of excitonic coupling effects on the absorbance and CD spectra of dye aggregates. We have followed the approach of Kühn, Renger, and May in which a dominant vibrational mode in each dye is singled out and treated as separate from the vibrational continuum and a judicious choice of basis states is made in which to express the system Hamiltonian. ${ }^{2-8}$ As these authors have pointed out: it should be emphasized that the expression for the system Hamiltonian corresponds to standard expressions commonly used in exciton-vibrational problems; the major difference, however, results from the fact that the representation of the theory in the vibrational eigenstate avoids any perturbation treatment of the excitonvibrational coupling. The KRM model allows one to efficiently obtain accurate eigenvalues and eigenvectors by diagonalizing the system Hamiltonian on suitably truncated Hilbert space. A limitation in the approach used here (but not a limitation of the KRM model) is that we have not included the coupling of the system Hamiltonian to the bath modes, which give rise to line broadening. Instead, for simplicity, we obtain a line broadened spectra by convolving the line spectra with a Gaussian. The width of the Gaussian is treated as a fitting parameter. For simplicity, the vibronic potential of the electronic excited state of a dye is taken to be a harmonic oscillator and the same as that for the electronic ground state except for a position offset. Here, a cluster of $\mathrm{N}$ dyes, of identical molecular structure, coupled vibrationally to the environment is considered. It is convenient to express the energy eigenstate of a dye $s$ as:

$$
|s ; \epsilon\rangle|s ; \epsilon, v\rangle,
$$


where $\mathrm{s}$ is the site (dye) index, $|\mathrm{s} ; \epsilon\rangle$ is the electronic energy eigenstate where $\epsilon=$ 0 for the ground state and $\epsilon=1$ for the excited state, and $|s ; \epsilon, v\rangle$ is the vibronic energy eigenstate where $v$ is an integer ranging from 0 to $\infty$ and is the number of quanta in the vibrational potential well of the electronic ground state when $\epsilon=0$ and the number of quanta in the vibrational potential well of the electronic excited state when $\epsilon=1$. The $|s, \epsilon\rangle$ are taken to be orthonormal, that is:

$$
\left\langle s ; \epsilon \mid s ; \epsilon^{\prime}\right\rangle=\delta_{\epsilon, \epsilon^{\prime}}
$$

In addition, the following orthonormality relations hold:

$$
\left\langle\mathrm{s} ; 0, \mathrm{v} \mid \mathrm{s} ; 0, \mathrm{v}^{\prime}\right\rangle=\left\langle\mathrm{s} ; 1, \mathrm{v} \mid \mathrm{s} ; 1, \mathrm{v}^{\prime}\right\rangle=\delta_{\mathrm{v}, \mathrm{v}^{\prime}}
$$

Because we have chosen a basis set where the vibrational energy eigenstates of the electronic excited state manifold differ from the vibrational basis set of the electronic ground state manifold, due to the horizontal displacement of the excited state vibronic potential from that of the ground state vibronic potential by the dimensionless amount $d$, these two sets of basis states are not orthogonal to each other. In particular, the overlap integrals between the vibrational eigenstates of the excited electronic state manifold and the electronic ground state manifold are given by:

$$
\left\langle s ; 0, v \mid s ; 1, v^{\prime}\right\rangle=\int_{-\infty}^{\infty} \varphi_{v}(x) \varphi_{v^{\prime}}(x-d) d x
$$

and

$$
\left\langle s ; 1, v \mid s ; 0, v^{\prime}\right\rangle=\int_{-\infty}^{\infty} \varphi_{v}(x-d) \varphi_{v^{\prime}}(x) d x
$$

where $\varphi_{\mathrm{v}}(\mathrm{x})$ is the wave function for the $v$ th energy eigenstate of the harmonic oscillator:

$$
\varphi_{\mathrm{v}}(\mathrm{x})=\frac{1}{\sqrt{2^{\mathrm{n}} \mathrm{n} ! \sqrt{\pi}}} \mathrm{e}^{-\frac{\mathrm{x}^{2}}{2}} \mathrm{H}_{\mathrm{v}}(\mathrm{x})
$$


and $H_{v}(x)$ is the vth Hermite polynomial. Despite this nonorthogonality, the states $|s ; \epsilon\rangle|s ; \epsilon, v\rangle$ form an orthonormal basis set due to Eq. (2.22), that is, one has:

$$
\left\langle s ; \epsilon, v\left|\left\langle s ; \epsilon \mid s ; \epsilon^{\prime}\right\rangle\right| s ; \epsilon^{\prime}, v^{\prime}\right\rangle=\delta_{\epsilon, \epsilon^{\prime}} \delta_{v, v^{\prime}}
$$

The Hilbert space for the system of $\mathrm{N}$ dyes is the outer product of the Hilbert spaces for the individual dyes and is spanned by the orthonormal basis set:

$$
\begin{aligned}
& \mathrm{B}_{1}= \\
& \left\{\prod_{\mathrm{s}=1}^{\mathrm{N}} \otimes\left|\mathrm{s} ; \epsilon_{\mathrm{s}}\right\rangle\left|\mathrm{s} ; \epsilon_{\mathrm{s}}, \mathrm{v}_{\mathrm{s}}\right\rangle \mid \forall_{\epsilon_{\mathrm{s}}} \in\{0,1\} \text { and } \mathrm{v}_{\mathrm{s}} \in\{0,1, \ldots \infty\}\right\}
\end{aligned}
$$

The Hamiltonian for the system of $\mathrm{N}$ dyes can now be written as:

$$
\begin{aligned}
& \mathrm{H}=\sum_{\mathrm{s}=1}^{\mathrm{N}} \mathrm{E}_{\mathrm{opt}}|\mathrm{s} ; 1\rangle\langle\mathrm{s} ; 1|+ \\
& \sum_{\mathrm{s}=1}^{\mathrm{N}} \sum_{\epsilon=0}^{1} \sum_{\mathrm{v}=0}^{\infty} \mathrm{vE}_{\mathrm{vib}}|\mathrm{s} ; \epsilon\rangle|\mathrm{s} ; \epsilon, v\rangle\langle\mathrm{s} ; \epsilon, v|\langle\mathrm{s} ; \epsilon|+ \\
& \sum_{\mathrm{s}=1}^{\mathrm{N}} \sum_{\substack{\mathrm{s}^{\prime}=1 \\
\mathrm{~s} \prime \neq s}}^{\mathrm{N}} \mathrm{J}_{\mathrm{s}, \mathrm{s}^{\prime}}|\mathrm{s} ; 1\rangle\left\langle\mathrm{s} ; 0|\otimes| \mathrm{s}^{\prime} ; 0\right\rangle\left\langle\mathrm{s}^{\prime} ; 1\right| .
\end{aligned}
$$

where $E_{\text {opt }}$ is the energy difference between the ground electronic state and the excited electronic state of a dye, $\mathrm{E}_{\mathrm{vib}}$, is the energy of a quantum of vibration, and $\mathrm{J}_{\mathrm{s}, \mathrm{s}}$, is the exchange energy between dyes $s$ and s' resulting from dipole-dipole coupling between transition dipoles. Since $\mathrm{J}_{\mathrm{s}, \mathrm{s}^{\prime}}=\mathrm{J}_{\mathrm{s}^{\prime}, \mathrm{s}}$, the Hamiltonian is manifestly Hermitian.

This Hamiltonian conserves the exciton number and consequently block diagonalizes according to exciton number. Hence, for low light conditions where the probability for the presence of more than one exciton in the dye cluster is small, one need only consider $\mathrm{H}$ on the one exciton sector of the Hilbert space. The orthonormal basis vectors spanning this space can be written in the form $\left|s ; v_{1}, v_{2}, \ldots v_{N}\right\rangle$ where the index $s$ now indicates which dye is excited and it is understood that $v_{s}$ is the number of quanta in 
the excited state of dye $\mathrm{s}$ and $\mathrm{v}_{\mathrm{i}}$ for $i \neq \mathrm{s}$ is the number of vibrational quanta in the ground state of dye $i$. In terms of the basis states S1, one has:

$$
\begin{aligned}
& \left|s ; v_{1}, v_{2}, \ldots v_{N}\right\rangle=\left(\prod_{s^{\prime}=1}^{s-1} \otimes\left|s^{\prime} ; 0\right\rangle\left|s^{\prime} ; 0, v_{s^{\prime}}\right\rangle\right) \otimes \\
& |s ; 1\rangle\left|s ; 1, v_{s}\right\rangle \otimes \prod_{s^{\prime}=s+1}^{N}\left|s^{\prime} ; 0\right\rangle\left|s^{\prime}, 0, v_{s^{\prime}}\right\rangle .
\end{aligned}
$$

Since the range of $\mathrm{v}_{\mathrm{i}}$ spans from 0 to $\infty$ in order to numerically diagonalize the Hamiltonian, approximations must be made. Often, this is accomplished by truncating the Hilbert space such that the $v_{i}$ are restricted over a finite range of integers: $0 \leq v_{i} \leq n_{\max }$. Since only the $\mathrm{v}_{\mathrm{s}}=0$ and $\mathrm{v}_{\mathrm{s}}=1$ vibronic peaks appear prominently in our absorption data, our fits to the absorption spectra were performed with $\mathrm{n}_{\max }=2$; that is, the $\mathrm{v}_{\mathrm{s}}=0,1$, and 2 vibronic states of each dye were employed.

Since Cy5 is a rod shaped molecule having greater length than width and since these molecules are brought in close proximity with each other where nearest-neighbor distances are comparable to the width of the molecules, the approximation of the exchange interaction as that resulting from two point dipoles was deemed insufficient. Instead, an expression for $\mathrm{J}_{\mathrm{s}, \mathrm{s}^{\prime}}$ was employed having the same form as that resulting from the coulomb interaction between point charges located near ends of the molecules. In particular, $\mathrm{J}_{\mathrm{s}, \mathrm{s}^{\prime}}$ was taken to be given by:

$$
\mathrm{J}_{\mathrm{s}, \mathrm{s}^{\prime}}=\mathrm{J}\left[\frac{1}{\left|\mathrm{r}_{\mathrm{s}}^{(1)}-\mathrm{r}_{\mathrm{s}^{\prime}}^{(1)}\right|}-\frac{1}{\left|\mathrm{r}_{\mathrm{s}}^{(1)}-\mathrm{r}_{\mathrm{s}^{\prime}}^{(2)}\right|}-\frac{1}{\left|\mathrm{r}_{\mathrm{s}}^{(2)}-\mathrm{r}_{\mathrm{s}^{\prime}}^{(1)}\right|}-\frac{1}{\mid \mathrm{r}_{\mathrm{s}}^{(2)}-\mathrm{r}_{\mathrm{s}^{\prime}}^{(2)}}\right]
$$

where $r_{s}^{(1)}$ and $r_{s}^{(2)}$ are the position vectors for the two opposite ends of dye s. J can be related to the charge $\delta$ of the point charges by $J=\delta^{2} / 4 \pi \varepsilon$. In the limit where the length $\mathrm{l}=\left|\mathrm{r}_{\mathrm{s}}^{(1)}-\mathrm{r}_{\mathrm{s}^{\prime}}^{(2)}\right|$ of the molecule goes to zero, the usual dipole-dipole form of the exchange interaction is recovered. 
The eigenvalues and eigenvectors of the Hamiltonian were computed using a software program written in-house. For the computation of the absorption and CD spectra, it was assumed that the initial state of the system was the ground state of the system; that is, the state in which there are no excitons present and the vibronic state is $\mathrm{v}=0$ (since at room temperature, the thermal energy $k T$ is small compared to the vibrational energy) for all of the dyes. This state can be simply denoted as $|0\rangle$. Let $\left|E_{\mathrm{i}}\right\rangle$ denote the ith energy eigenstate of the Hamiltonian, then the transition rate $\gamma_{i}$ from the ground state to the energy eigenstate $\mathrm{i}$ for the case when the dye complexes are randomly oriented in solution is given by:

$$
\gamma_{\mathrm{i}}=\beta\left|\left\langle\mathrm{E}_{\mathrm{i}}|\mu| 0\right\rangle\right|^{2}
$$

where $\beta$ is a proportionality constant and the transition amplitude dipole moment $\mu$ is given by:

$$
\begin{aligned}
& \left\langle E_{\mathrm{i}}|\mu| 0\right\rangle \\
& =\sum_{\mathrm{s}=1}^{\mathrm{N}} \sum_{\mathrm{v}_{\mathrm{s}}=1}^{\mathrm{N}} \mu \mathrm{n}_{\mathrm{s}} \sum_{\mathrm{v}_{\mathrm{s}}=0}^{\infty}\left\langle\mathrm{E}_{\mathrm{i}} \mid \mathrm{s} ; 0,0, \ldots, \mathrm{v}_{\mathrm{s}}, 0, \ldots 0\right\rangle\left\langle\mathrm{s} ; 1, \mathrm{v}_{\mathrm{s}} \mid \mathrm{s} ; 0,0\right\rangle,
\end{aligned}
$$

where $n_{s}$ is the orientation vector for the transition dipole of the dye $s$.

The difference, $\gamma_{\mathrm{i}}^{\mathrm{CD}}$, in the transition rates induced by right-handed and lefthanded circularly polarized light is given by: 


$$
\begin{aligned}
& \gamma_{\mathrm{i}}^{\mathrm{CD}} \\
& =\frac{4 \pi \beta}{\lambda_{\mathrm{i}}} \sum_{\substack{\mathrm{s}=1 \\
\mathrm{~s}^{\prime}=1 \\
\mathrm{~s} \neq \mathrm{s}^{\prime}}}^{\mathrm{N}}\left(\mathrm{n}_{\mathrm{s}} \times \mathrm{n}_{\mathrm{s}^{\prime}}\right) \cdot\left(\mathrm{R}_{\mathrm{s}}-\mathrm{R}_{\mathrm{s}^{\prime}}\right) \\
& \times \sum_{\mathrm{v}_{\mathrm{s}}=0}^{\infty}\left\langle\mathrm{E}_{\mathrm{i}} \mid \mathrm{s} ; 0,0, \ldots, \mathrm{v}_{\mathrm{s}}, 0, \ldots 0\right\rangle\left\langle\mathrm{s}^{\prime} ; 1, \mathrm{v}_{\mathrm{s}} \mid \mathrm{s} ; 0,0\right\rangle \\
& \times \sum_{\mathrm{v}_{\mathrm{s}^{\prime}}=0}^{\infty}\left\langle\mathrm{E}_{\mathrm{i}} \mid \mathrm{s}^{\prime} ; 0,0, \ldots, \mathrm{v}_{\mathrm{s}^{\prime}}, 0, \ldots 0\right\rangle\left\langle\mathrm{s}^{\prime} ; 1, \mathrm{v}_{\mathrm{s}^{\prime}} \mid \mathrm{s}^{\prime} ; 0,0\right\rangle,
\end{aligned}
$$

where $R_{s}$ is the position vector to the center of dye $s$, and $\lambda_{i}$ is the wavelength of light for which the quantum of energy is $E_{i}=h c / \lambda_{i}$.

The absorbance as a function of energy E, for comparison with experimental data, was computed as:

$$
A(E)=\sum_{i} \frac{\gamma_{i}}{\sqrt{2 \pi \Gamma^{2}}} \exp \left(-\frac{\left(E-E_{i}\right)^{2}}{2 \Gamma^{2}}\right) .
$$

Similarly, the differential CD absorbance as a function of energy was computed as:

$$
A_{C D}(E)=\sum_{i} \frac{\gamma_{i}^{C D}}{\sqrt{2 \pi \Gamma^{2}}} \exp \left(-\frac{\left(E-E_{i}\right)^{2}}{2 \Gamma^{2}}\right),
$$

where the value of $\Gamma$ was adjusted to give the best fit to all the data.

The fit parameters used are listed in Table 2.4. By first fitting the monomer spectra, we could determine $\mathrm{E}_{\mathrm{vib}}(0.16 \mathrm{eV})$ and the horizontal displacement $\mathrm{d}(0.78)$, which were constants used for fitting the aggregate spectra. To fit the J-dimer and $\mathrm{H}$ tetramer data, we considered the length of the dye molecule to be $1.4 \mathrm{~nm}$, the damping constant $(\Gamma)$ due to the energy loss to be $0.038 \mathrm{eV}$, and number of vibronic states $\left(\mathrm{n}_{\mathrm{v}}\right)$ to be 3 . 
The resulting outputs of the fit provide information pertaining to the angles and position of the dyes relative to each other. Given in spherical coordinates, the zenith $(\theta)$ and azimuth $(\varphi)$ angles are given in degrees. The Cartesian components of the orientation vector for a dye are given in terms of $\theta$ and $\varphi$ by the following set of equations:

$$
\begin{aligned}
& \mathrm{n}_{\mathrm{x}}=\sin (\theta) \cos (\varphi), \\
& \mathrm{n}_{\mathrm{y}}=\sin (\theta) \sin (\varphi), \\
& \mathrm{n}_{\mathrm{z}}=\cos (\theta) .
\end{aligned}
$$

The positions of the dyes are given in angstroms and listed in Table 2.5.

As a measure the goodness of the fits, we evaluated the overlap integrals of the experimental spectra with the theoretical spectra. Letting $S_{a b, e x}(E), S_{a b, t h}(E), S_{c d, e x}(E)$, and $\mathrm{S}_{\mathrm{cd}, \mathrm{th}}(\mathrm{E})$ denote respectively the experimental absorbance spectrum, theoretical absorbance spectrum, experimental CD spectrum, and theoretical CD spectrum where E is energy, the normalized absorbance overlap integral $\left(\mathrm{OI}_{\mathrm{AB}}\right)$ of the spectrum is defined by

$$
\mathrm{OI}_{\mathrm{AB}}=\frac{\int \mathrm{S}_{\mathrm{ab}, \mathrm{ex}}(\mathrm{E}) \mathrm{S}_{\mathrm{ab}, \mathrm{th}}(\mathrm{E}) \mathrm{dE}}{\sqrt{\int \mathrm{S}_{\mathrm{ab}, \mathrm{ex}}^{2}(\mathrm{E}) \mathrm{dE}} \sqrt{\int \mathrm{S}_{\mathrm{ab}, \mathrm{th}}^{2}(\mathrm{E}) \mathrm{dE}}} .
$$

and the normalized overlap integral for the $\mathrm{CD}$ spectrum $\left(\mathrm{OI}_{\mathrm{CD}}\right)$ is defined by

$$
\mathrm{OI}_{\mathrm{CD}}=\frac{\int \mathrm{S}_{\mathrm{cd}, \mathrm{ex}}(\mathrm{E}) \mathrm{S}_{\mathrm{cd}, \mathrm{th}}(\mathrm{E}) \mathrm{dE}}{\sqrt{\int \mathrm{S}_{\mathrm{cd}, \mathrm{ex}}^{2}(\mathrm{E}) \mathrm{dE}} \sqrt{\int \mathrm{S}_{\mathrm{cd}, \mathrm{th}}^{2}(\mathrm{E}) \mathrm{dE}}} .
$$

As an overall goodness parameter $\left(\mathrm{OI}_{\mathrm{Tot}}\right)$, we introduce

$$
\mathrm{OI}_{\mathrm{Tot}}=\frac{1}{2}\left(\mathrm{OI}_{\mathrm{ab}}+\mathrm{OI}_{\mathrm{cd}}\right) \text {. }
$$


The normalized overlap integrals provide a measure of how closely the experimental and theoretical curves match and are less than or equal to one. Only for a perfect match are they equal to one.

To obtain a better approximation for the exchange interaction (J) between dyes than the dipole-dipole coupling of two point dipoles provide, the exchange interaction [Eq. (2.21)] was used. In this form of the exchange interaction, the dipoles are modeled as two point charges, of opposite sign, separated by a distance, $\mathrm{l}_{\mathrm{c}}$. This distance was taken to be $1.4 \mathrm{~nm}$, which is shorter than the physical length of the Cy5 molecule (1.6 $\mathrm{nm}$ ) in order to account for the fact that the physical charge distribution is spread out over the length of the molecule but with the maxima of the charge density occurring near, but not necessarily at, the ends of the molecule. Additionally, a physical constraint was placed on the fit to force the minimum distance between dye molecules to maintain a 0.45 nm separation.

Table 2.4 Kühn -Renger-May model fit parameters for the J-dimer and Htetramer absorbance and CD spectra.

\begin{tabular}{|c|c|c|c|c|c|}
\hline Dye-DNA & \multicolumn{5}{|c|}{ Kühn-Renger-May Model Parameters } \\
Construct & \multicolumn{5}{|c|}{} \\
\hline & $n_{v}$ & $E_{o}(\mathrm{eV})$ & $J\left(\mathrm{eV} \mathrm{nm}^{3}\right)$ & $\Gamma(\mathrm{eV})$ & $O I_{T o t}$ \\
\hline J-Dimer & 3 & 0.0 & 0.054 & 0.038 & 0.984001 \\
\hline H-Tetramer & 3 & 0.1805 & 0.054 & 0.038 & 0.972912 \\
\hline
\end{tabular}


Table 2.5 Kühn-Renger-May model fitting outputs describing the dye orientations and positions.

\begin{tabular}{|c|c|c|c|c|c|}
\hline Dye-DNA & \multicolumn{5}{|c|}{ Kühn-Renger-May Model Parameters } \\
Construct & \multicolumn{5}{|c|}{} \\
\hline & $\theta$ (degrees) & $\varphi$ (degrees) & $x(\AA)$ & $y(\AA)$ & $z(\AA)$ \\
\hline$\underline{J-D i m e r}$ & & & & & \\
Dye 1 & $43.5^{\circ}$ & $180^{\circ}$ & 0.0 & 0.75135 & 7.5 \\
Dye 2 & $43.5^{\circ}$ & $0^{\circ}$ & 0.0 & -0.75135 & -7.5 \\
\hline$\underline{\text { H-Tetramer }}$ & & & & & \\
Dye 1 & $7.78^{\circ}$ & $2.07^{\circ}$ & 2.83431 & 1.93217 & -0.89778 \\
Dye 2 & $6.24^{\circ}$ & $91.07^{\circ}$ & 3.45277 & -7.50334 & -3.31306 \\
Dye 3 & $8.70^{\circ}$ & $180.77^{\circ}$ & 2.19294 & 10.97340 & 2.256089 \\
Dye 4 & $0.81^{\circ}$ & $269.87^{\circ}$ & 2.25385 & 6.46436 & 1.531691 \\
\hline
\end{tabular}

As a method for substantiating $\mathrm{H}$-Tetramer fit data, a series of fits with various dye orientations and configurations were executed and shown in Figure 2.8. By comparing all variations of the fits to the H-tetramer spectra, one can eliminate the "less good" fits, thereby substantiating the goodness of the best fit and gives confidence to the dye stacking arrangement that we have proposed in the manuscript. Quantitatively, the fits are considered poor due to overlap integral values $\left(\mathrm{OI}_{\mathrm{Tot}}\right)$ that differ from 1 by more than 5\% (Table 2.6) and qualitatively do not correspond well to the curve of the experimental data. Output parameters of the poor alternative fits are provided in Table 2.7 . 
a
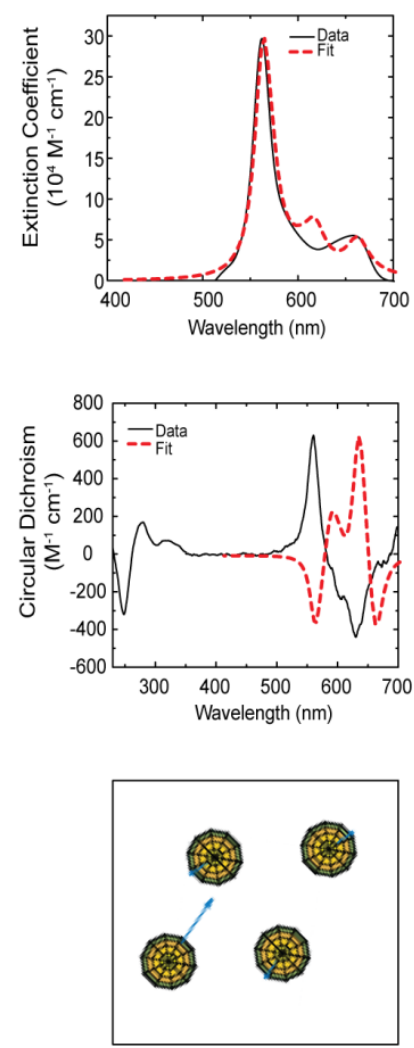

b
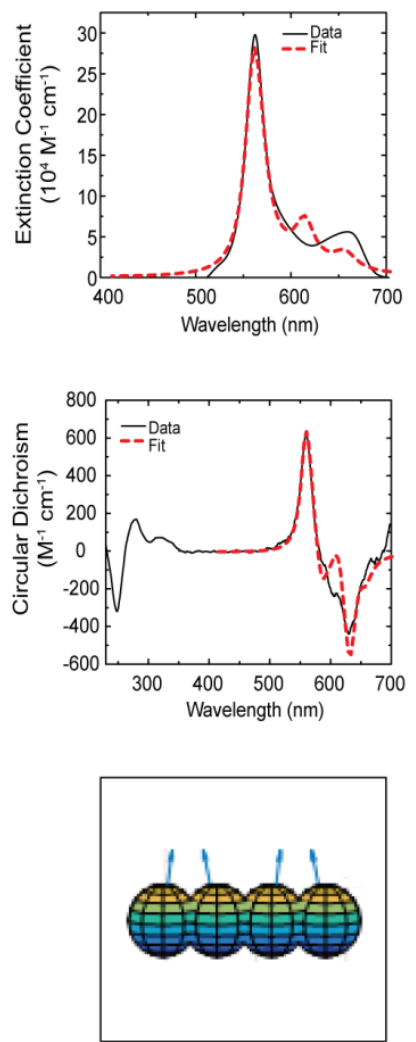

C
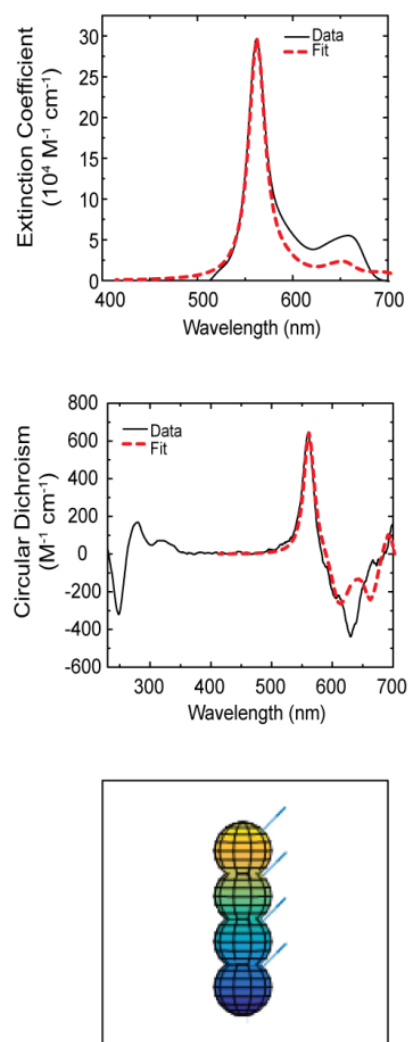

Figure 2.8. Three poor alternative fits of the H-tetramer absorbance (top panels) and CD (middle panels) spectra using the Kühn-Renger-May model to quantitatively compare the goodness of fit for a variety of dye configurations (bottom panels), including (a) square, (b) in line, and (c) stacked configurations.

Table 2.6 Kühn-Renger-May model fit parameters for the poor alternative fits of the H-tetramer absorbance and CD spectra shown in Figure 2.8.

\begin{tabular}{|c|c|c|c|c|c|}
\hline Dye-DNA & \multicolumn{5}{|c|}{ Kühn-Renger-May Model Parameters } \\
Construct & \multicolumn{5}{|c|}{} \\
\hline & $n_{v}$ & $E_{o}(\mathrm{eV})$ & $J\left(\mathrm{eV} \mathrm{nm}^{3}\right)$ & $\Gamma(\mathrm{eV})$ & $O I_{T o t}$ \\
\hline Square & 3 & 0.050 & 0.054 & 0.038 & 0.191103014 \\
\hline Line & 3 & -0.070 & 0.054 & 0.038 & 0.924583964 \\
\hline Stacked & 3 & -0.075 & 0.054 & 0.038 & 0.926914204 \\
\hline
\end{tabular}


Table 2.7 Kühn-Renger-May model fitting outputs describing the dye orientations and positions of the poor alternative fits for the $\mathrm{H}$ tetramer absorbance and CD spectra shown in Figure 2.8.

\begin{tabular}{|c|c|c|c|c|c|}
\hline Dye-DNA & \multicolumn{5}{|c|}{ Kühn-Renger-May Model Parameters } \\
Construct & \multicolumn{5}{|c|}{} \\
\hline & $\theta$ (degrees) & $\varphi$ (degrees) & $x(\AA)$ & $y(\AA)$ & $z(\AA)$ \\
\hline Square & & & & & \\
Dye 1 & $20^{\circ}$ & $65^{\circ}$ & -3.0 & 3.0 & 0.0 \\
Dye 2 & $60^{\circ}$ & $245^{\circ}$ & 5.0 & 5.0 & 0.0 \\
Dye 3 & $20^{\circ}$ & $45^{\circ}$ & 3.0 & -3.0 & 0.0 \\
Dye 4 & $20^{\circ}$ & $225^{\circ}$ & -5.0 & -5.0 & 0.0 \\
\hline Line & & $-10^{\circ}$ & 0.0 & 0.0 & 0.0 \\
Dye 1 & $45^{\circ}$ & $-13^{\circ}$ & 0.0 & 4.5 & 0.0 \\
Dye 2 & $45^{\circ}$ & $-10^{\circ}$ & 0.0 & 9.0 & 0.0 \\
Dye 3 & $45^{\circ}$ & $-13^{\circ}$ & 0.0 & 13.5 & 0.0 \\
Dye 4 & $45^{\circ}$ & $-25^{\circ}$ & 0.0 & 13.5 & 0.0 \\
\hline Stacked & & $45^{\circ}$ & 0.0 & 4.5 & 0.0 \\
Dye 1 & $10^{\circ}$ & $45^{\circ}$ & 0.0 & 0.0 & 0.0 \\
Dye 2 & $-20^{\circ}$ & $35^{\circ}$ & 0.0 & 9.0 & 0.0 \\
Dye 3 & $10^{\circ}$ & $45^{\circ}$ & & & \\
Dye 4 & -2.0 & & \\
\hline
\end{tabular}




\subsubsection{Detailed Derivation of Second-Order Reactions Kinetics Model}

Here, we provide a more detailed view of the second-order reaction kinetics derivation. From Eq. (2.1) we are given the following second-order chemical reaction:

$$
J+J \underset{k_{H \rightarrow J}}{\stackrel{k_{J \rightarrow H}}{\rightleftharpoons} \mathrm{H}}
$$

such that the rate equations for the system are:

$$
\frac{d[J]}{d t}=-2 k_{J \rightarrow H}[J]^{2}+2 k_{H \rightarrow J}[H]
$$

and

$$
\frac{d[H]}{d t}=k_{J \rightarrow H}[J]^{2}-k_{H \rightarrow J}[H]
$$

Note that, per conservation of mass, we have:

$$
[H]=\frac{1}{2}([D N A]-[J]) .
$$

Thus, while conserving mass, we can rewrite Eq. (2.43) as:

$$
\frac{d[H]}{d t}=k_{J \rightarrow H}([D N A]-2[H])^{2}-k_{H \rightarrow J}[H]
$$

or

$$
\begin{aligned}
& \quad \frac{d[H]}{d t}=4 k_{J \rightarrow H}[H]^{2}-\left(4 k_{J \rightarrow H}[D N A]+k_{H \rightarrow J}\right)[H]+ \\
& k_{J \rightarrow H}[D N A]^{2}
\end{aligned}
$$

This differential equation can be cast into the integral form:

$$
\int_{[H]_{0}}^{[H]} \frac{d x}{f+g x+h x^{2}}=t
$$

where

$$
\begin{aligned}
& f=k_{J \rightarrow H}[D N A]^{2} . \\
& g=-4 k_{J \rightarrow H}[D N A]-k_{H \rightarrow J}
\end{aligned}
$$




$$
h=4 k_{J \rightarrow H}
$$

Additionally, the following is introduced:

$$
r=-4 f h+g^{2}=8 k_{J \rightarrow H} k_{H \rightarrow J}[D N A]+k_{H \rightarrow J}^{2} .
$$

Note, that $r$ is equivalent to $q$ defined in Eq. (2.9). Using the CRC table of integrals, Eq. (2.47) becomes:

$$
\left.\frac{1}{\sqrt{q}} \ln \left(\frac{2 h x+g-\sqrt{q}}{2 h x+g+\sqrt{q}}\right)\right|_{x=[H]_{0}} ^{x=[H]},
$$

which yields,

$$
\ln \left(\frac{2 h[H]+g-\sqrt{q}}{2 h[H]+g+\sqrt{q}}\right)=\ln (\beta)+\sqrt{q} t,
$$

Where:

$$
\beta=\frac{2 h[H]_{0}+g-\sqrt{q}}{2 h[H]_{0}+g+\sqrt{q}}
$$

Simplifying by eliminating the natural log,

$$
\frac{2 h[H]+g-\sqrt{q}}{2 h[H]+g+\sqrt{q}}=\beta e^{\sqrt{q} t}
$$

and solving for $[H]$ yields,

$$
[H]=\frac{\sqrt{q}}{2 h}\left(\frac{1+\beta e^{\sqrt{q} t}}{1-\beta e^{\sqrt{q} t}}\right)-\frac{g}{2 h} .
$$

Given the following relationships, as provided in Eq. (2.8),

$$
\begin{aligned}
& a=-k_{H \rightarrow J}[D N A], \\
& b=k_{H-J}, \\
& c=2 k_{J \rightarrow H} .
\end{aligned}
$$

We can re-express the relations:

$$
\frac{g}{2 h}=-\frac{1}{2}[D N A]-\frac{k_{H \rightarrow J}}{8 k_{J \rightarrow H}} .
$$

obtained from Eq. (2.49) and Eq. (2.50) as: 


$$
\frac{g}{2 h}=-\frac{1}{2}[D N A]-\frac{b}{4 c} .
$$

Similarly, Eq. (2.50) can be expressed as:

$$
h=2 c .
$$

Therefore, we can now rewrite Eq. (2.56) as:

$$
[H]=\frac{1}{2}[D N A]+\frac{\sqrt{q}}{4 c}\left(\frac{1+\beta e^{\sqrt{q} t}}{1-\beta e^{\sqrt{q} t}}\right)+\frac{b}{4 c} .
$$

Additionally, rearranging Eq. (2.54), we find that:

$$
\beta=\frac{4 c[H]_{0}+2 c[D N A]-b-\sqrt{q}}{4 c[H]_{0}+2 c[D N A]-b+\sqrt{q}},
$$

Conserving mass and eliminating $[H]_{0}$ and $[D N A]$ from the equation yields:

$$
\beta=\frac{2 c[J]_{0}+b+\sqrt{q}}{2 c[J]_{0}+b-\sqrt{q}}
$$

which can be related to Eq. (2.10) as:

$$
\beta=\frac{1}{\alpha} .
$$

Therefore, the final equation can be given as follows:

$$
[H]=\frac{1}{2}[D N A]+\frac{\sqrt{q}}{4 c}\left(\frac{1+\alpha e^{-\sqrt{q} t}}{1-\alpha e^{-\sqrt{q} t}}\right)+\frac{b}{4 c},
$$

which is provided in Eq. (2.7).

\subsubsection{Reaction Kinetics}

The first method by which the reaction kinetics data were fit is described in Eq.

(2.14). The rate constants are determined by substituting the extracted parameters into the following two equations:

$$
\begin{aligned}
& q=8 k_{J \rightarrow H} k_{H \rightarrow J}[D N A]+k_{H \rightarrow J}^{2}, \\
& k_{J \rightarrow H}=K_{e q} * k_{H \rightarrow J} .
\end{aligned}
$$


where $[D N A]$ represents the total concentration of DNA after injection, $k_{J \rightarrow H}$, and $k_{H \rightarrow J}$ represent the reaction rate constants for J-dimers transitioning into H-tetramers (i.e., forward reaction) and H-tetramers transitioning into J-dimers (i.e., reverse reaction), respectively, and $K_{e q}$ is the equilibrium constant [Eq. (73)]. Representative data sets of raw and normalized reaction kinetics obtained as a function of time are shown in Figures 2.9 and 2.10, respectively. Note that reproduced data is not shown but was acquired to provide error bars on the rate constants (shown in Figure 2.4e,f), which were determined using the standard deviation and a Python-based error propagation calculator.

Fitting parameters and regression analysis results are listed in Tables 2.8 and 2.9. The calculated rate constants, found using Eqs. (2.70) and (2.71), are listed in Tables 2.10 and 2.11. Note that the $K_{e q}$ values determined by fitting the absorption spectra in Figure 2.17 are also listed in Tables 2.10 and 2.11. The second method for fitting the reaction kinetics data is to assume that when $t$ is small $(t \approx 0)$ the initial concentration of $\mathrm{J}$ dimers $([J])$ at high initial salt concentration is approximately zero and thus the reaction will follow a first-order rate law, such that:

$$
k_{H \rightarrow J}=\frac{\ln (2)}{t_{1 / 2}}
$$

where $t_{1 / 2}$ is the time when the reaction will have reached half-completion. With this, it then follows that:

$$
k_{J \rightarrow H}=k_{H \rightarrow J} K_{e q}
$$

The value of the equilibrium constant $K_{e q}$ is found by fitting the absorbance spectra in Figure 2.17. The rate constants and corresponding equilibrium constants are found using this method and are listed in Table 2.12. Finding the equilibrium constants 
independently provides confidence for our rate constant values extracted from the reaction kinetics data using a second-order reaction model.

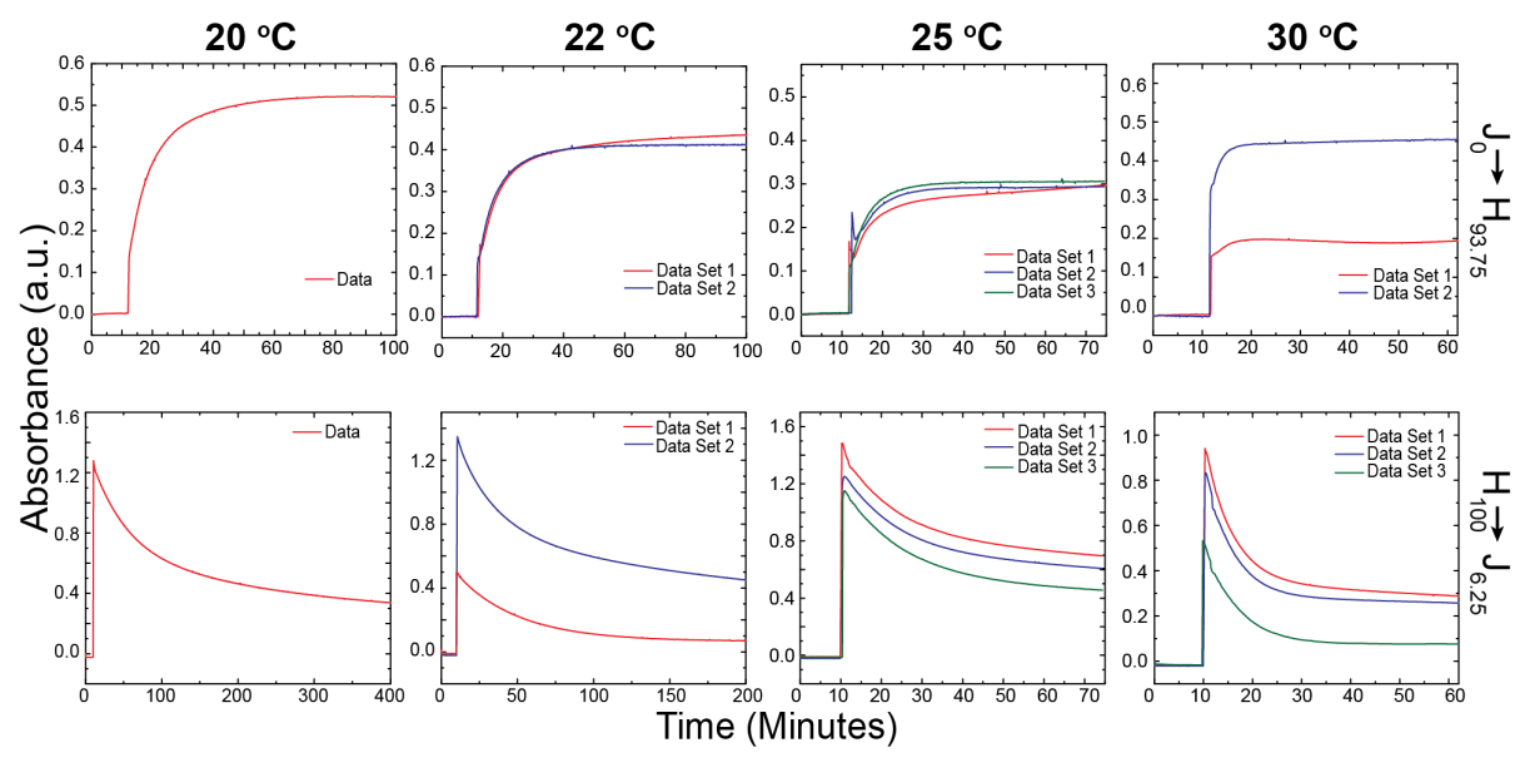

Figure 2.9. Raw (i.e., non-normalized) reaction kinetics data demonstrating aggregate state transitions at $20^{\circ} \mathrm{C}, 22^{\circ} \mathrm{C}, 25^{\circ} \mathrm{C}$, and $30{ }^{\circ} \mathrm{C}$. The dye-DNA constructs $(1.25 \mu \mathrm{M}$ DNA concentration) were monitored at $562 \mathrm{~nm}$, the H-tetramer peak absorption maximum, in order to eliminate interference from the monomer. The first 10-12 minutes of the data examines any baseline absorption due to the TAE buffer, after which the dye-DNA constructs were added into the solution. The top row of panels (Jo-H93.75) show switching from the J-dimer state initially at $\mathbf{0 ~} \mathrm{mM} \mathrm{MgCl}_{2}$ to predominantly H-tetramer states at a final salt concentration of $93.75 \mathbf{m M ~ M g C l}$. The bottom row of panels ( $\left.\mathrm{H}_{100} \mathrm{~J}_{6.25}\right)$ demonstrate switching from H-tetramers initially at $100 \mathrm{mM} \mathrm{MgCl} 2$ to mainly J-dimers at a final salt concentration of $6.25 \mathrm{mM}$ $\mathrm{MgCl}_{2}$. Small spikes evident in the data at the time of injection are the result of momentarily inserting a pipette into the incident light beam path. 


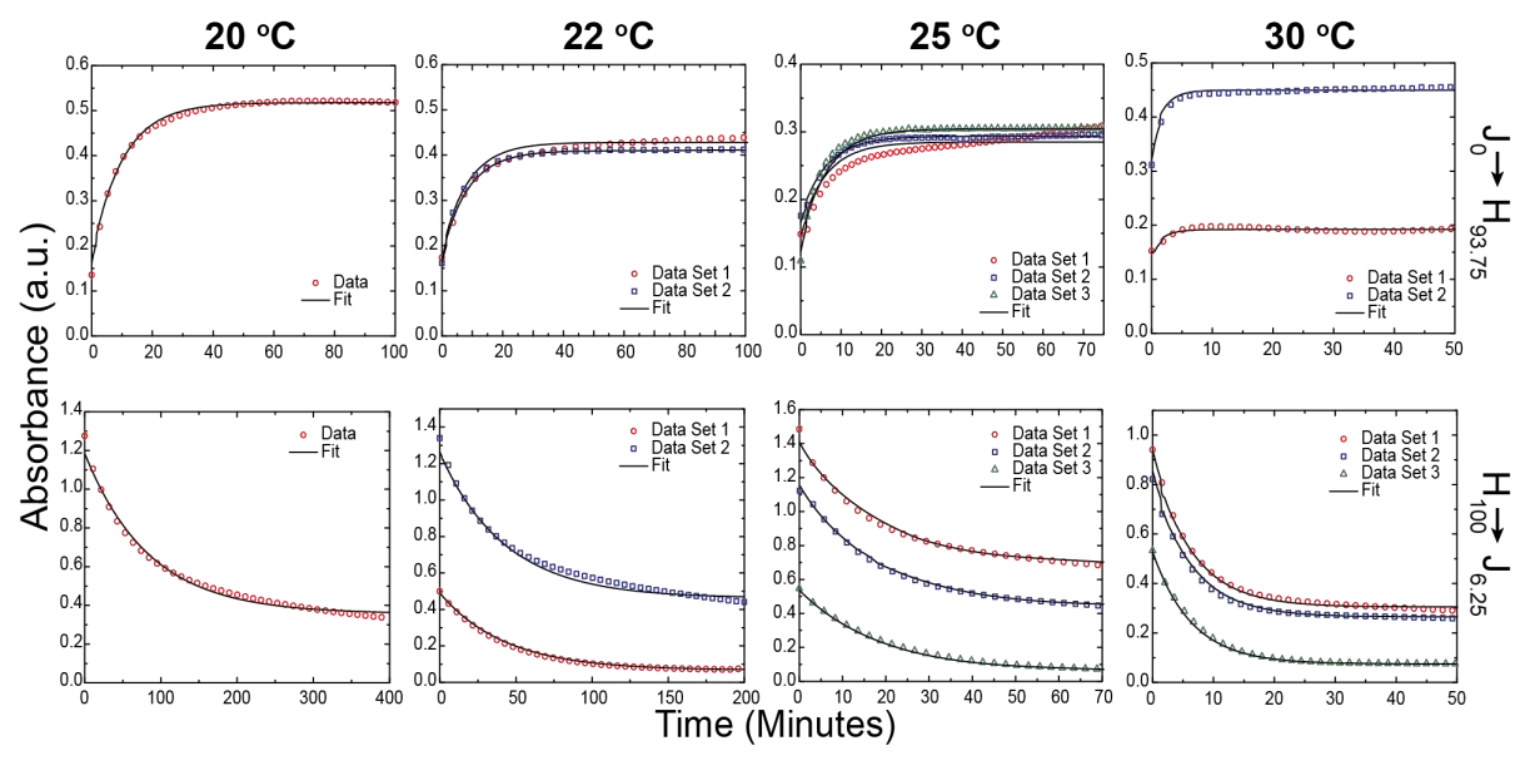

Figure 2.10. Reaction kinetics data demonstrating J-dimer to H-tetramer (top row of panels) and H-tetramer to J-dimer (bottom row of panels) transitions. The black curves shown in each plot represent the fit used to calculate the second-order reaction rate constants. Fitting parameters and regression coefficient parameters are listed in Tables 2.8 and 2.9. Reaction rate constants are calculated from the fits are listed in Table 2.12. 
Table 2.8 Reaction kinetics fitting parameters obtained for each data set shown in the top row of panels in Figure 2.10. All dye-DNA constructs were at $1.25 \mu \mathrm{M}$ DNA concentration with $93.75 \mathrm{mM}$ added $\mathrm{MgCl}_{2}$. The number following the temperature describes the corresponding data set for which the fit was applied.

\begin{tabular}{|c|c|c|c|c|c|}
\hline \multirow[t]{2}{*}{$\begin{array}{c}J \rightarrow H \\
\text { Data }\end{array}$} & \multicolumn{3}{|c|}{$[H]=\left([H]_{t=0}-[H]_{t=\infty}\right)\left(\frac{(1}{1}\right.$} & $\begin{array}{l}\text { lation } \\
\left.\frac{\sqrt{q} t}{\bar{q} t}\right)+\end{array}$ & $+[H]_{t=\infty}$ \\
\hline & {$[H]_{t=0}$ (a.u.) } & $\begin{array}{c}{[H]_{t=\infty}} \\
\text { (a.u.) }\end{array}$ & $\alpha$ & $q$ & Adjusted $\mathrm{R}^{2}$ \\
\hline $20^{\circ} \mathrm{C}, 1$ & 0.1615 & 0.518 & $6.8 \times 10^{-6}$ & 0.009 & 0.995 \\
\hline $22^{\circ} \mathrm{C}, 1$ & 0.1714 & 0.428 & $5.5 \times 10^{-4}$ & 0.009 & 0.980 \\
\hline $22^{\circ} \mathrm{C}, 2$ & 0.2569 & 0.522 & $5.5 \times 10^{-4}$ & 0.018 & 0.996 \\
\hline $25^{\circ} \mathrm{C}, 1$ & 0.1479 & 0.293 & $4.7 \times 10^{-4}$ & 0.008 & 0.948 \\
\hline $25^{\circ} \mathrm{C}, 2$ & 0.166 & 0.264 & $4.7 \times 10^{-4}$ & 0.021 & 0.973 \\
\hline $25^{\circ} \mathrm{C}, 3$ & 0.1236 & 0.305 & $4.7 \times 10^{-4}$ & 0.041 & 0.997 \\
\hline $30^{\circ} \mathrm{C}, 1$ & 0.2148 & 0.192 & $6.1 \times 10^{-3}$ & 0.317 & 0.772 \\
\hline $30^{\circ} \mathrm{C}, 2$ & 0.3213 & 0.450 & $6.1 \times 10^{-3}$ & 0.216 & 0.969 \\
\hline
\end{tabular}


Table 2.9 Reaction kinetics fitting parameters obtained for each data set shown in the bottom row of panels in Figure 2.10. All dye-DNA constructs were at $1.25 \mu \mathrm{M}$ DNA concentration with $6.25 \mathrm{mM}$ added $\mathrm{MgCl}_{2}$. The number following the temperature describes the corresponding data set for which the fit was applied.

\begin{tabular}{|c|c|c|c|c|c|}
\hline \multirow{2}{*}{$\begin{array}{c}\boldsymbol{H} \rightarrow \boldsymbol{J} \\
\text { Data }\end{array}$} & \multicolumn{4}{|c|}{$[H]=\left([H]_{t=0}-[H]_{t=\infty}\right)\left(\frac{(1-\alpha) e^{-\sqrt{q} t}}{1-\alpha e^{-\sqrt{q} t}}\right)+[H]_{t=\infty}$} \\
\hline & {$[H]_{t=0}$ (a.u.) } & $\begin{array}{c}{[H]_{t=\infty}} \\
\text { (a.u.) }\end{array}$ & $\alpha$ & Adjusted $\mathrm{R}^{2}$ \\
\hline $20^{\circ} \mathrm{C}, 1$ & 1.185 & 0.357 & $8.2 \times 10^{-10}$ & 0.0001 & 0.993 \\
\hline $22^{\circ} \mathrm{C}, 1$ & 1.263 & 0.462 & $2.2 \times 10^{-4}$ & 0.0005 & 0.992 \\
\hline $22^{\circ} \mathrm{C}, 2$ & 0.490 & 0.067 & $2.2 \times 10^{-4}$ & 0.0006 & 0.999 \\
\hline $25^{\circ} \mathrm{C}, 1$ & 0.534 & 0.056 & $2.0 \times 10^{-4}$ & 0.0025 & 0.999 \\
\hline $25^{\circ} \mathrm{C}, 2$ & 1.410 & 0.687 & $2.0 \times 10^{-4}$ & 0.0034 & 0.995 \\
\hline $25^{\circ} \mathrm{C}, 3$ & 1.153 & 0.439 & $2.0 \times 10^{-4}$ & 0.0032 & 0.991 \\
\hline $25^{\circ} \mathrm{C}, 4$ & 0.537 & 0.060 & $2.0 \times 10^{-3}$ & 0.0027 & 0.999 \\
\hline $30^{\circ} \mathrm{C}, 1$ & 0.940 & 0.305 & $7.1 \times 10^{-3}$ & 0.0241 & 0.998 \\
\hline $30^{\circ} \mathrm{C}, 2$ & 0.854 & 0.265 & $7.1 \times 10^{-3}$ & 0.0271 & 0.999 \\
\hline $30^{\circ} \mathrm{C}, 3$ & 0.528 & 0.075 & $7.1 \times 10^{-3}$ & 0.0221 & 0.999 \\
\hline
\end{tabular}


Table 2.10 Calculated forward $\left(k_{J \rightarrow H}\right)$ and reverse $\left(k_{H \rightarrow J}\right)$ reaction rate constants for the $\mathrm{J}_{0}-\mathrm{H}_{93.75}$ reactions shown in the upper row of panels in Figure 2.10. all dye-DNA constructs were at $1.25 \mu \mathrm{M}$ DNA concentration with $93.75 \mathrm{mM}$ added $\mathrm{MgCl}_{2} . K_{e q}$ values were found by fitting the absorbance data in Figure 2.17.

\begin{tabular}{|c|c|c|c|}
\hline $\begin{array}{c}\text { Averaged Rate } \\
\text { Constant }\end{array}$ & $\boldsymbol{k}_{\boldsymbol{J} \rightarrow \boldsymbol{H}}(\mathrm{M} \mathrm{s})^{-1}$ & $\boldsymbol{k}_{\boldsymbol{H} \rightarrow \boldsymbol{J}} 10^{-2}(\mathrm{~s})^{-1}$ & $\boldsymbol{K}_{\boldsymbol{e q}}(\mathrm{M})^{-1}$ \\
\hline $20^{\circ} \mathrm{C}$ & $1.7 \pm 0.1$ & $1.6 \pm 0.3$ & 1,050 \\
\hline $22^{\circ} \mathrm{C}$ & $17.8 \pm 4.2$ & $1.8 \pm 0.4$ & 9,800 \\
\hline $25^{\circ} \mathrm{C}$ & $21.1 \pm 8.2$ & $2.3 \pm 0.9$ & 9,100 \\
\hline $30^{\circ} \mathrm{C}$ & $268.1 \pm 36.1$ & $7.3 \pm 1.0$ & 36,600 \\
\hline
\end{tabular}

Table 2.11 Calculated forward $\left(k_{J \rightarrow H}\right)$ and reverse $\left(k_{H \rightarrow J}\right)$ reaction rate constants for the $\mathrm{H}_{100}-\mathrm{J}_{6.25}$ reactions shown in the bottom row panels in Figure 2.10. All dye-DNA constructs were at $1.25 \mu \mathrm{M}$ DNA concentration with $6.25 \mathrm{mM}$ added $\mathrm{MgCl}_{2}$. $K_{e q}$ values were found by fitting the absorbance data in Figure 2.17.

\begin{tabular}{|c|c|c|c|}
\hline $\begin{array}{c}\text { Averaged Rate } \\
\text { Constant }\end{array}$ & $\boldsymbol{k}_{\boldsymbol{J} \rightarrow \boldsymbol{H}} 10^{-5}(\mathrm{M} \mathrm{s})^{-1}$ & $\boldsymbol{k}_{\boldsymbol{H} \rightarrow \boldsymbol{J}} 10^{-4}(\mathrm{~s})^{-1}$ & $\boldsymbol{K}_{\text {eq }} 10^{-2}(\mathrm{M})^{-1}$ \\
\hline $20^{\circ} \mathrm{C}$ & $1.0 \pm 0.1$ & $2.0 \pm 0.8$ & 5.3 \\
\hline $22^{\circ} \mathrm{C}$ & $2.2 \pm 0.2$ & $3.9 \pm 0.3$ & 5.6 \\
\hline $25^{\circ} \mathrm{C}$ & $8.5 \pm 0.6$ & $9.0 \pm 0.6$ & 9.4 \\
\hline $30^{\circ} \mathrm{C}$ & $22.7 \pm 1.2$ & $26.0 \pm 7.8$ & 8.7 \\
\hline
\end{tabular}


Table 2.12 Calculated forward $\left(k_{J \rightarrow H}\right)$ and reverse $\left(k_{H \rightarrow J}\right)$ reaction rate constants and corresponding equilibrium constants for $\mathrm{H}_{100-} \mathrm{J}_{6.25}$ obtained using the second method, see Eqs. (2.28) and (2.29). All dye-DNA constructs were at $1.25 \mu \mathrm{M}$ DNA concentrations with $6.25 \mathrm{mM}$ added $\mathrm{MgCl}_{2}$. $K_{e q}$ values were found by fitting the absorbance data in Figure 2.17.

\begin{tabular}{|c|c|c|c|}
\hline Rate Constant & $\boldsymbol{k}_{\boldsymbol{J} \rightarrow \boldsymbol{H}} 10^{-5}(\mathrm{M} \mathrm{s})^{-1}$ & $\boldsymbol{k}_{\boldsymbol{H} \rightarrow \boldsymbol{J}} 10^{-4}(\mathrm{~s})^{-1}$ & $\boldsymbol{K}_{\boldsymbol{e q}} 10^{-2}(\mathrm{M})^{-1}$ \\
\hline $20^{\circ} \mathrm{C}$ & 1.3 & 2.5 & 5.3 \\
\hline $22^{\circ} \mathrm{C}$ & 2.4 & 4.4 & 5.6 \\
\hline $25^{\circ} \mathrm{C}$ & 9.8 & 10.4 & 9.4 \\
\hline $30^{\circ} \mathrm{C}$ & 22.7 & 25.7 & 8.7 \\
\hline
\end{tabular}

\subsubsection{Spectral Analysis of Absorbance and Fluorescence Data}

UV-Vis absorbance and fluorescence spectra for each dye-DNA construct was analyzed by fitting each dataset to a series of Gaussian curves. From the Gaussian curves, the peak positions and full width at half maximums (FWHMs) were obtained. The Gaussian fit is defined as:

$$
y=y_{0}+\left(\frac{A}{w \sqrt{\frac{\pi}{2}}}\right) e^{-2\left(\frac{x-x_{c}}{w}\right)^{2}}
$$

where $y_{0}$ is an offset in terms of either $\mathrm{mM}^{-1}$ or counts. $\mathrm{mM}^{-1}$ for absorbance or fluorescence, respectively. The area of the curve is given as $A$, the position of the peak is denoted by $x_{c}$, and the width of the peak is given by $w$. The FWHMs are calculated as $w \sqrt{\ln (4)}$ and listed in Table 2.13. Gaussian fit parameters and residuals are provided in Table 2.13 for each dye-DNA construct's absorbance (Abs) and fluorescence (FL) peak maximum. 

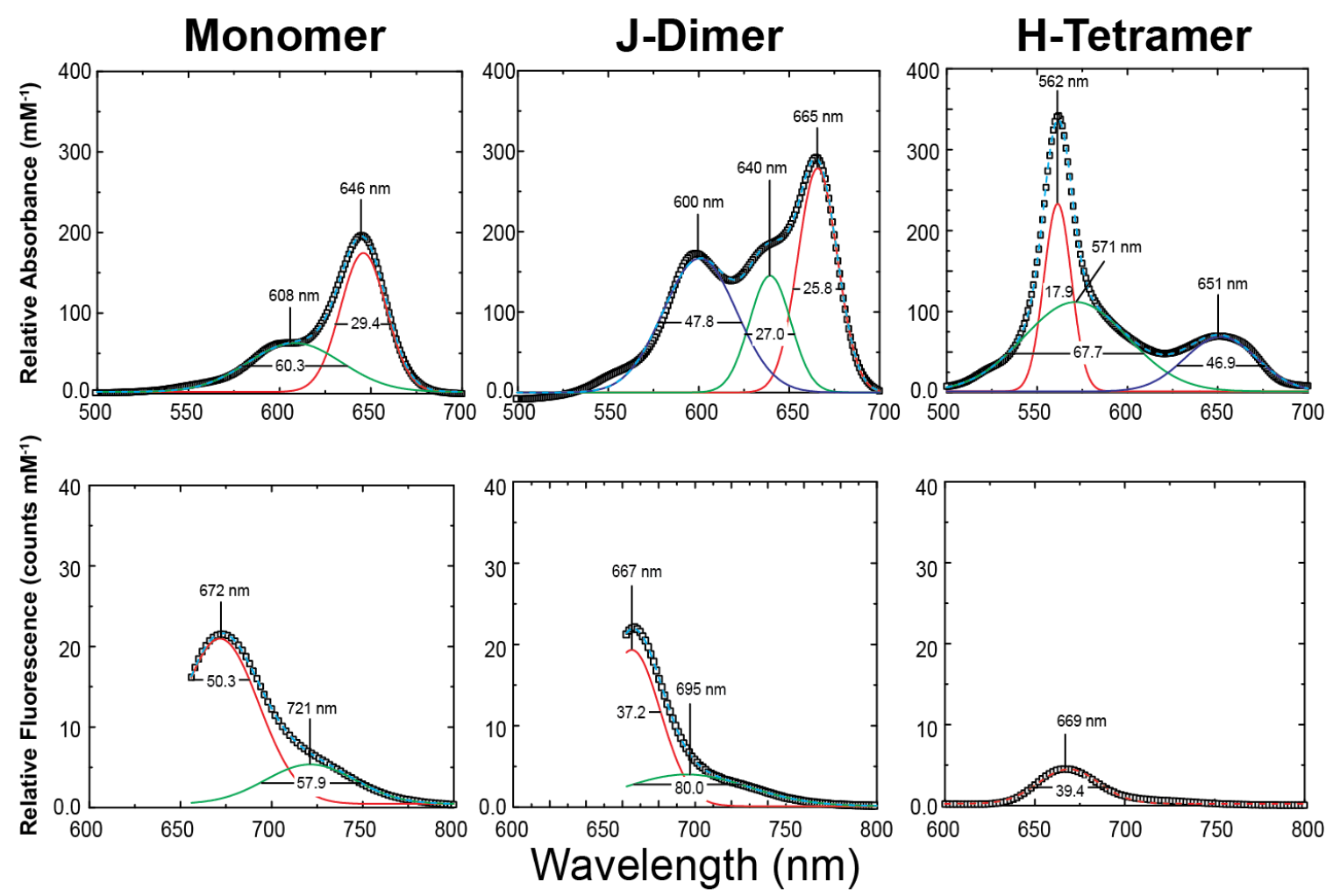

Figure 2.11. Relative UV-Vis absorbance spectra (top panels) and fluorescence (bottom panels) of the monomer $\left(15 \mathrm{mM}\right.$ added $\left.\mathrm{MgCl}_{2}\right)$, J-dimer $(0 \mathrm{mM}$ added $\mathrm{MgCl}_{2}$ ), and $\mathrm{H}$-tetramer ( $155 \mathrm{mM}$ added $\mathrm{MgCl}_{2}$ ) from left to right, respectively. Each dataset was fit to a series of Gaussian curves to obtain information regarding the peak positions and full width at half maximums (FWHMs). All solutions of dye-DNA constructs were prepared at $1.5 \mu \mathrm{M}$. 
Table 2.13 Gaussian fitting parameters obtained for the peak maximum of each absorbance (Abs) and fluorescence (FL) data set shown in Figure 2.11.

\begin{tabular}{|c|c|c|c|c|c|c|}
\hline & \multicolumn{7}{|c|}{ Gaussian Fit Equation } \\
$\begin{array}{c}\text { Dye-DNA } \\
\text { Construct }\end{array}$ & \multicolumn{7}{|c|}{$y=y_{0}+\left(\frac{A}{w \sqrt{\frac{\pi}{2}}}\right) e^{-2\left(\frac{x-x_{c}}{w}\right)^{2}}$} \\
\hline & $\mathrm{y}_{0}$ & $x_{c}(\mathrm{~nm})$ & $w(\mathrm{~nm})$ & $A$ & $\begin{array}{c}\text { Adjusted } \\
\mathrm{R}^{2}\end{array}$ & $\begin{array}{c}\text { FWHM } \\
(\mathrm{nm})\end{array}$ \\
\hline $\begin{array}{c}\text { Monomer } \\
(\text { Abs })\end{array}$ & $\begin{array}{c}(2.3 \pm \\
0.1) \times 10^{3}\end{array}$ & $\begin{array}{c}645.9 \pm \\
0.1\end{array}$ & $25.0 \pm 0.1$ & $\begin{array}{c}(5.4 \pm \\
0.1) \times 10^{3}\end{array}$ & 0.999 & $29.4 \pm 0.2$ \\
\hline $\begin{array}{c}\text { Monomer } \\
(\mathrm{FL})\end{array}$ & $\begin{array}{c}(4.7 \pm \\
0.3) \times 10^{4}\end{array}$ & $\begin{array}{c}671.7 \pm \\
0.2\end{array}$ & $42.7 \pm 0.3$ & $\begin{array}{c}(1.1 \pm \\
0.1) \times 10^{8}\end{array}$ & 0.999 & $50.3 \pm 0.4$ \\
\hline $\begin{array}{c}\text { J-Dimer } \\
(\text { Abs })\end{array}$ & $0.0 \pm 0.0$ & $\begin{array}{c}665.8 \pm \\
6.2\end{array}$ & $21.9 \pm 7.4$ & $\begin{array}{c}(7.6 \pm \\
4.4) \times 10^{6}\end{array}$ & 0.138 & $25.8 \pm 8.7$ \\
\hline $\begin{array}{c}\text { J-Dimer } \\
(\mathrm{FL})\end{array}$ & $\begin{array}{c}(1.1 \pm \\
0.8) \times 10^{4}\end{array}$ & $\begin{array}{c}667.1 \pm \\
0.1\end{array}$ & $31.6 \pm 0.1$ & $\begin{array}{c}(7.6 \pm \\
0.6) \times 10^{7}\end{array}$ & 0.999 & $37.2 \pm 0.1$ \\
\hline $\begin{array}{c}\text { H-Tetramer } \\
(\mathrm{Abs})\end{array}$ & $\begin{array}{c}(1.4 \pm \\
0.7) \times 10^{3}\end{array}$ & $\begin{array}{c}561.4 \pm \\
0.1\end{array}$ & $15.2 \pm 0.1$ & $\begin{array}{c}(4.4 \pm \\
0.4) \times 10^{6}\end{array}$ & 0.999 & $17.9 \pm 0.1$ \\
\hline $\begin{array}{c}\mathrm{H}-\mathrm{Tetramer} \\
(\mathrm{FL})\end{array}$ & $\begin{array}{c}(2.4 \pm \\
0.3) \times 10^{4}\end{array}$ & $\begin{array}{c}668.8 \pm \\
0.3\end{array}$ & $33.5 \pm 0.6$ & $\begin{array}{c}(1.8 \pm \\
0.3) \times 10^{7}\end{array}$ & 0.981 & $39.4 \pm 0.7$ \\
\hline
\end{tabular}

\subsubsection{Structural Characterization}

To assess the validity of the H-tetramer hypothesis as discussed in the manuscript, structural characterization of the J-dimer and $\mathrm{H}$-tetramer was performed using native poly-acrylamide gel electrophoresis (PAGE) to separate the structures based on their electrophoretic mobility and to compare the mobility of the J-dimer and H-tetramer against a variety of duplex and multi-arm structural controls (Figure 2.12). Multi-arm junctions were prepared in a similar manner to the duplex samples as described in section 2.8.1. Equimolar amounts of strands were mixed together in a $1 \times$ TAE buffer with either 0 or $100 \mathrm{mM}$ added $\mathrm{MgCl}_{2}$. Samples were allowed to hybridize at room temperature over a 24 hour period. Samples of DNA duplexes and multi-arm junctions at $10 \mu \mathrm{M}$ concentration with 0 or $100 \mathrm{mM}$ added $\mathrm{MgCl}_{2}$ were mixed in a 5:1 ratio with $6 \times \mathrm{New}$ 
England Biolabs loading buffer ( $1 \times$ buffer: $11 \mathrm{mM}$ ethylenediaminetetraacetic acid, 3.3 $\mathrm{mM}$ tris-hydrochloric acid, $0.017 \%$ sodium dodecyl sulfate, $0.015 \%$ bromophenol blue, $2.5 \%$ Ficoll@-400), injected into a 12\% native PAGE gel $(10 \mathrm{~cm}$ length and $1.5 \mathrm{~mm}$ width), and allowed to run for approximately 90-120 minutes with a 75-150 V applied voltage. To avoid introducing external intercalants or dyes into the system, staining agents were not used. Instead, completed PAGE gel runs were placed on a covered phosphor plate and imaged using a multiplexed gel imaging and documentation system (FluorChemQ, ProteinSimple). Gels were excited with a $254 \mathrm{~nm}$ UV light source and bands were identified by the shadows cast onto the illuminated phosphor plate. Bands containing well-formed dye-DNA constructs were excised from the gels and crushed using a micro-mortar and pestle. Constructs were extracted from the gel fragments by eluting the DNA in a $1 \times$ TAE buffer solution with 0 or $100 \mathrm{mM}$ added $\mathrm{MgCl}_{2}$.

(a) Three-Armed Junction

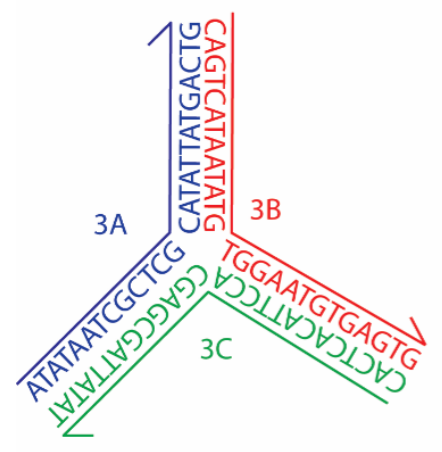

(b) Four-Armed Junction

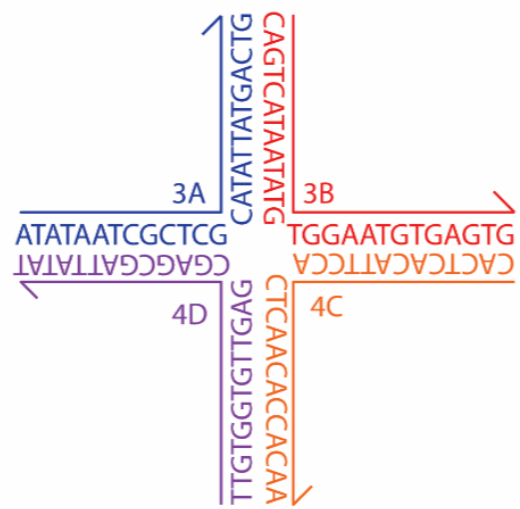

(c) Six-Armed Junction

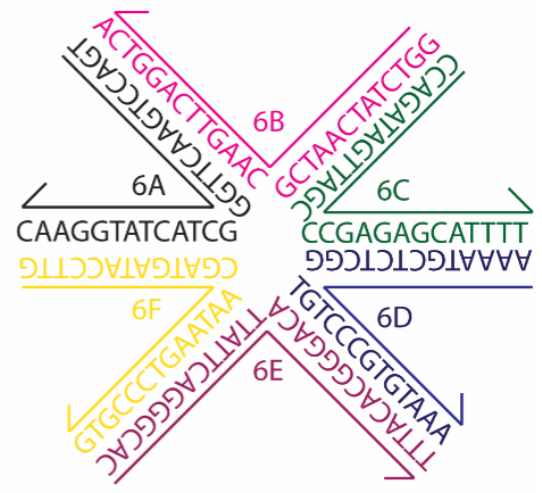

Figure 2.12. Illustrations of completed (a) three, (b) four, and (c) six-armed structures that were used as controls for monitoring the migration of duplexed strands during gel electrophoresis. Strand sequences are listed in Table 2.2.

A Cary 5000 UV-Vis-NIR spectrophotometer (Agilent Technologies) was used to acquire absorbance spectra of the dye-DNA constructs. Because Markova et al. used sodium chloride $(\mathrm{NaCl})$ as the main electrolyte source in their solution, ${ }^{34}$ we monitored 
changes in the UV-Vis absorbance spectra of the dye-DNA constructs for a variety of $\mathrm{NaCl}$ concentrations (Figure 2.13). Spectral scans were obtained by placing $150 \mu \mathrm{L}$ if sample into a self-masking, sub-micro, $1 \mathrm{~cm}$ path length quartz spectrophotometer cell (Starna). The initial DNA sample contained complementary dye-labeled oligomers (Cy50 and cCy5-0) in a $1 \times$ TAE buffer with no added $\mathrm{NaCl}$. Subsequently, $5 \mu \mathrm{L}$ aliquots of a $5 \mathrm{M} \mathrm{NaCl}$ solution were added sequentially to the solution. Upon completion, spectra were normalized by DNA concentration to take into account dilution effects. Note that some samples may not have had time to fully reach chemical equilibrium. Regardless, Figure 2.13 indicates that the Davydov splitting remains constant with increasing $\mathrm{NaCl}$ concentration, while the peak intensity ratio between the J-dimer $(665 \mathrm{~nm})$ and $\mathrm{H}$ tetramer $(562 \mathrm{~nm})$ constructs changes, similar to varying $\mathrm{MgCl}_{2}$ concentration. Differences in $\mathrm{NaCl}$ and $\mathrm{MgCl}_{2}$ concentrations may be attributed to differences in the ionic valencies between the sodium $\left(\mathrm{Na}^{+}\right)$ions and the magnesium $\left(\mathrm{Mg}^{2+}\right)$ ions, which also corresponds to a difference in the ionic strength. 


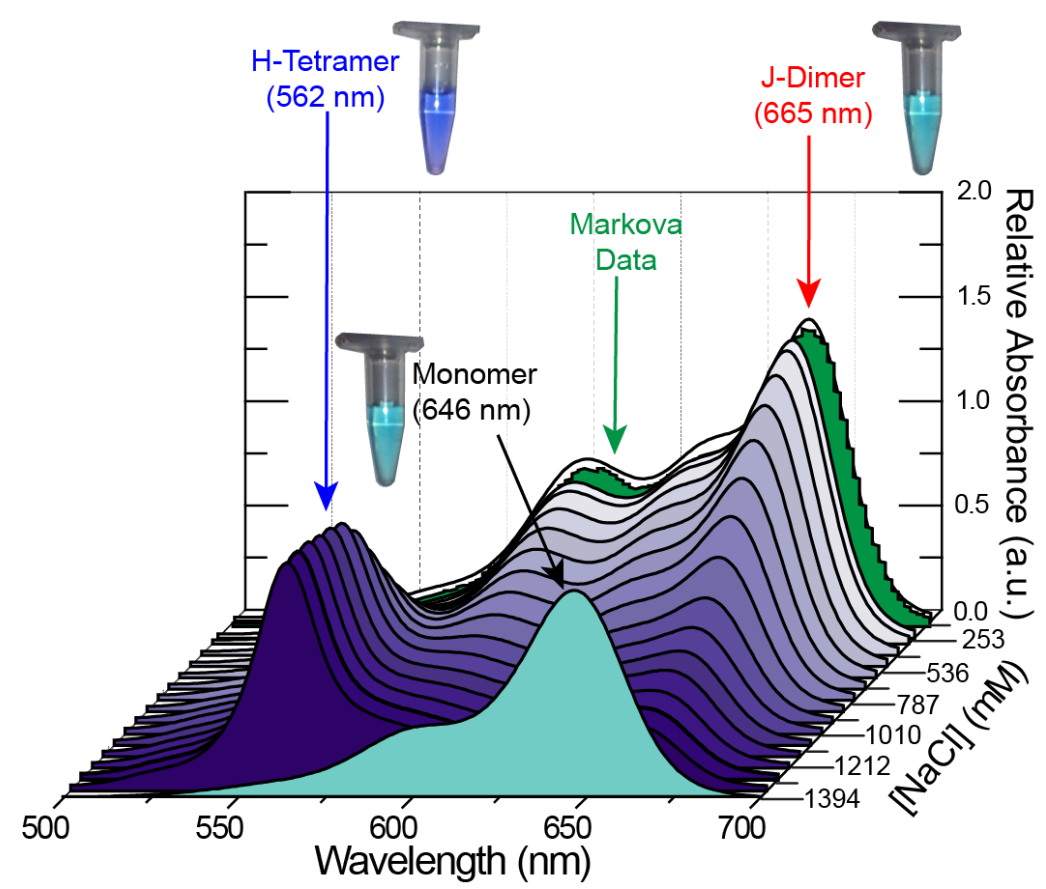

Figure 2.13. UV-Vis absorbance spectra normalized by DNA concentration showing spectral changes as a function of salt $(\mathrm{NaCl})$ concentration. Note that the scans were not necessarily taken at chemical equilibrium, but still indicate that a two-state quantum coherent system exists. The absorbance spectrum acquired by Markova $e t$ al. has been inserted into the data set at the appropriate salt concentration to demonstrate reproducibility.

Table 2.14 Equilibrium constant, $K_{e q}$, and fit regression coefficients, $R^{2}$. Samples were held at room temperature with $15 \mathrm{mM}$ added $\mathrm{MgCl}_{2}$. DNA concentrations were varied.

\begin{tabular}{|c|c|c|}
\hline & $\boldsymbol{K}_{\boldsymbol{e q}}\left(\mathbf{M}^{-1}\right)$ & $\boldsymbol{R}^{\mathbf{2}}$ \\
\hline$\frac{[J]}{[J]+2[H]}$ & $(5.1 \pm 1.6) 10^{1}$ & 0.931 \\
\hline$\frac{2[H]}{[J]+2[H]}$ & $(5.1 \pm 1.6) 10^{1}$ & 0.931 \\
\hline
\end{tabular}

\subsubsection{Calculation of Gibb's Free Energy $\left(\Delta \mathrm{G}^{\circ}\right)$}

To calculate the Gibbs free energy $\left(\Delta G^{\circ}\right)$ of the $\mathrm{J}_{0} \rightarrow \mathrm{H}_{93.75}$ and $\mathrm{H}_{100} \rightarrow \mathrm{J}_{6.25}$ reactions, the populations of the J-dimers $([J])$ and H-tetramers $([H])$ were calculated using Eq. (2.21). Subsequently, from Eq. (2.1), the following equation can be derived, 


$$
K_{e q}=\frac{[H]}{[J]^{2}}
$$

such that $\Delta G^{\circ}$ is found using the following equation:

$$
\Delta G^{\circ}=-R T \ln \left(\frac{[H]}{[J]^{2}}\right)
$$

where $R$ is the gas constant and $T$ is room temperature in Kelvin.

The values of $[J]$ and $[H]$ were calculated by taking absorbance spectra of the reaction kinetics samples after allowing the dye-DNA constructs to reach chemical and thermal equilibrium, as shown in Figure 2.14. The spectra were then fit to Eq. (2.21) to show the relative populations of $\mathrm{J}$ - and $\mathrm{H}$-aggregates and monomers in each sample (Figure 2.15). The populations at each temperature were then averaged to give an effective population for each specific reaction (i.e., $\mathbf{J}_{0} \rightarrow \mathrm{H}_{93.75}$ versus $\mathrm{H}_{100} \rightarrow \mathbf{J}_{6.25}$ ) and substituted into Eq. (2.25).

The calculated values for $K_{e q}$, found by fitting the data provided in Figure 2.15, are listed in Tables 2.10 and 2.11 . The $\Delta G^{\circ}$ values found at room temperature $\left(22^{\circ} \mathrm{C}\right)$ are listed in Table 2.1. 

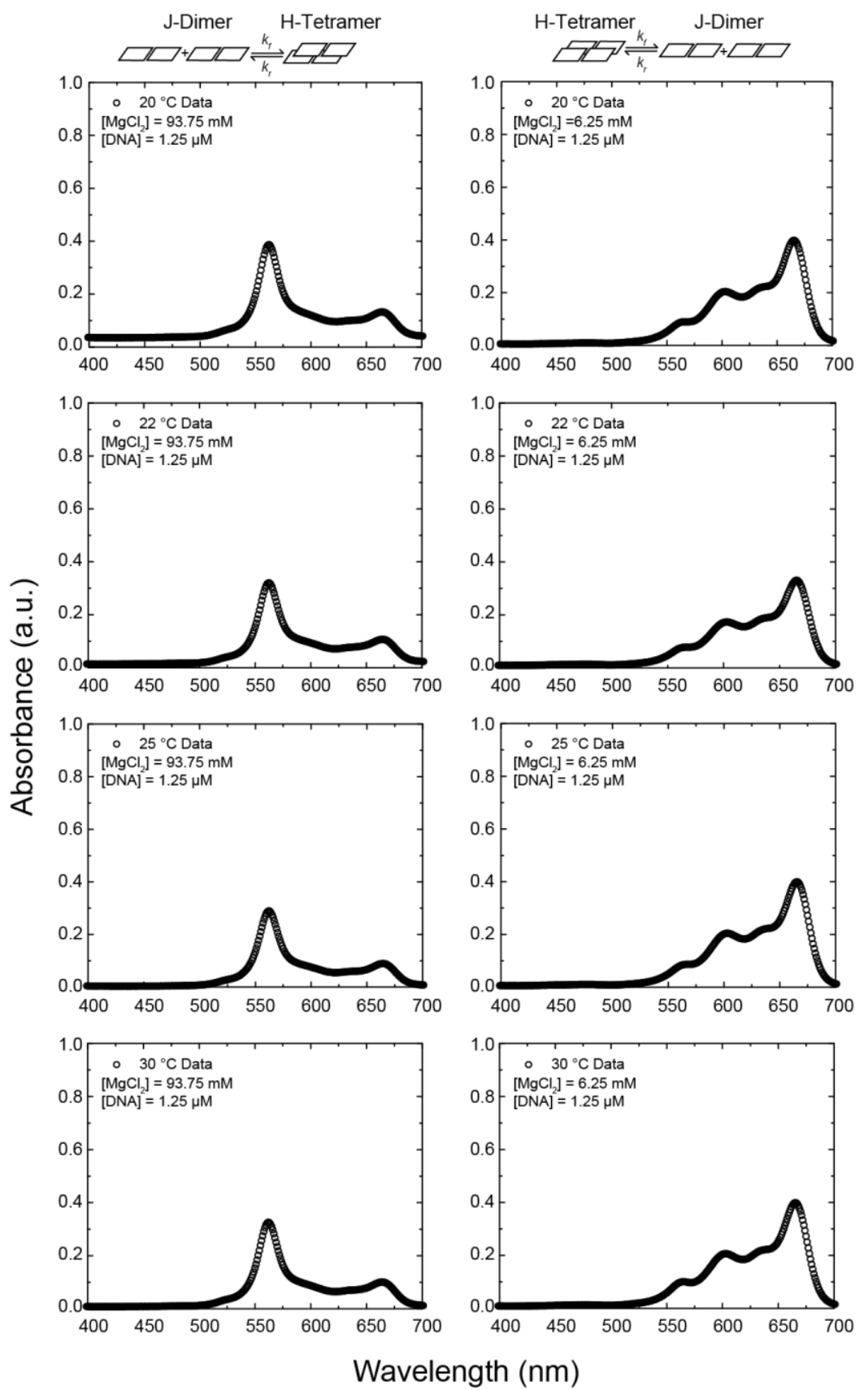

Figure 2.14. Raw UV-Vis absorbance spectra taken after completed reaction kinetics runs for both $\mathrm{J}_{0}-\mathrm{H}_{93.75}$ reactions (left side panel) and $\mathrm{H}_{100}-\mathrm{J}_{6.25}$ reactions (right side panel). All solutions of dye-DNA constructs consisted of $1.25 \mu \mathrm{M}$ DNA concentrations. Panels on the left were taken at $93.75 \mathrm{mM} \mathrm{MgCl}_{2}$ concentrations, while the panels on the right were taken at $6.25 \mathrm{mM} \mathrm{MgCl} 2$ concentration. 


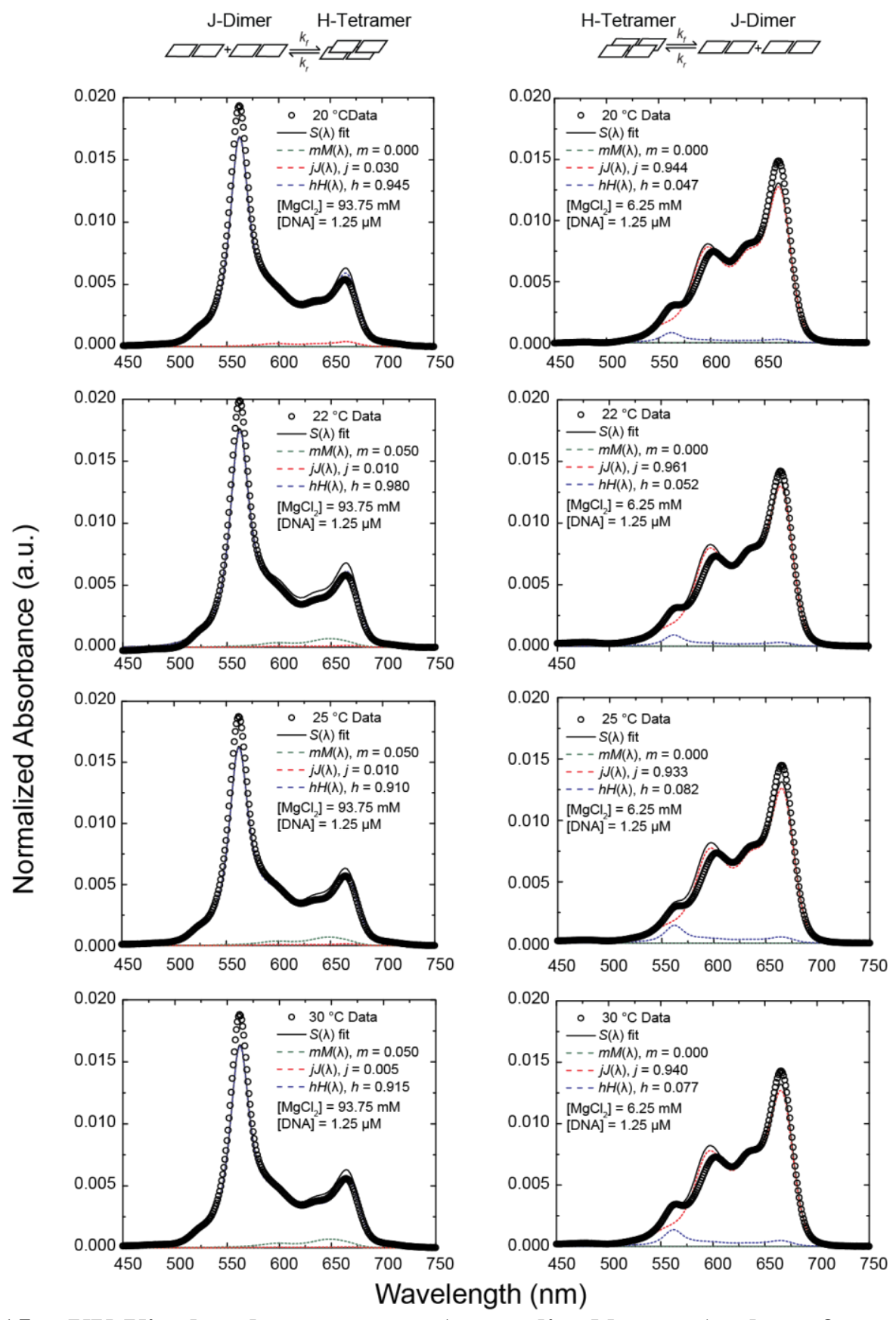

Figure 2.15. UV-Vis absorbance spectra (normalized by area) taken after completed reaction kinetics runs for both $\mathrm{J}_{0} \rightarrow \mathrm{H}_{93.75}$ reactions (left side panel) and $\mathrm{H}_{100} \rightarrow \mathrm{J}_{6.25}$ reactions (right side panel). Each scan was fit to Eq. (2.21) to give relative percentages of each individual aggregate state: $m$ (monomer), $j$ (J-dimer), and $h$ (H-tetramer). All solutions of dye-DNA constructs consisted of $1.25 \mu \mathrm{M}$ DNA concentrations. Panels on the left were taken at $93.75 \mathrm{mM} \mathrm{MgCl} 2$ concentrations, while the panels on the right were taken at $6.25 \mathrm{mM} \mathrm{MgCl}_{2}$ concentration. 


\subsubsection{Salt and DNA Concentration Studies}

PAGE purified samples of the dye-DNA monomer (i.e., duplexed DNA with a single Cy5 dye-labeled strand, M), J-dimer (J), and H-tetramer $(\mathrm{H})$ constructs were used to collect representative UV-Vis spectra for each pure aggregate. As shown in Figure 2.16, the spectra were acquired from 450-750 $\mathrm{nm}$ and normalized by area.

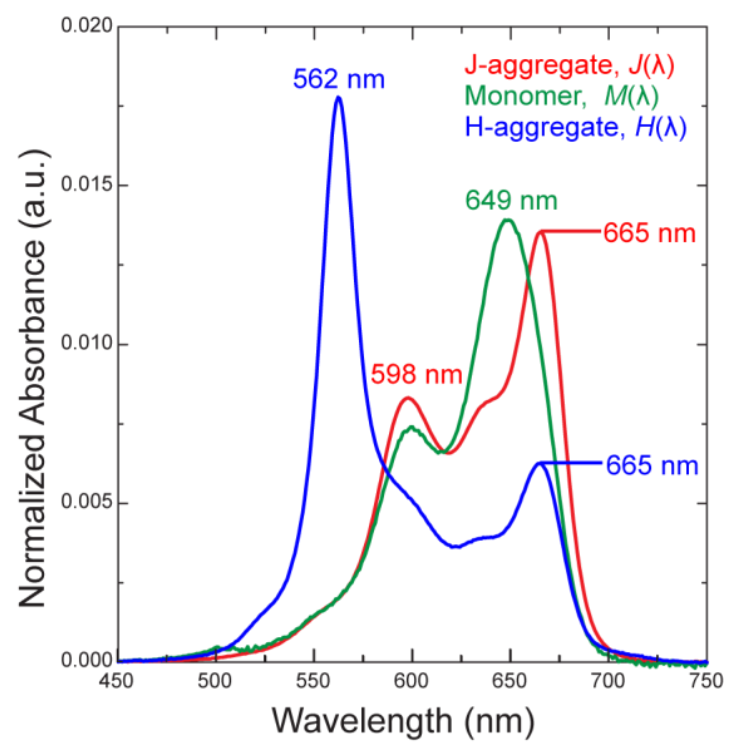

Figure 2.16. UV-Vis absorbance spectra of PAGE purified monomer $(M(\lambda)$; green curve), J-dimer $(J(\lambda)$; red curve), and $H$-tetramer $(H(\lambda)$; blue curve). all spectra have been normalized by area and subsequently used to determine the percentages of each state present in non-purified samples.

Using the control spectra obtained as described in the section above, the following fitting function that decomposes the spectrum into a linear combination of the UV-Vis spectrum for each individual dye-DNA construct (i.e., Monomer, J-dimer, or H-tetramer) was used:

$$
S(\lambda)=m \cdot M(\lambda)+j \cdot J(\lambda)+h \cdot H(\lambda)
$$

where the constants $m, j$, and $h$ are determined by a least squares fitting procedure using the control spectra $M(\lambda), J(\lambda)$, and $H(\lambda)$, which represent the monomer, J-dimer, and H-tetramer states, respectively. 
The concentration of monomer within the spectrum is a result of volumetric and concentration errors of non-purified samples. It is considered nearly negligible in all cases and not considered within any of the further calculations. 


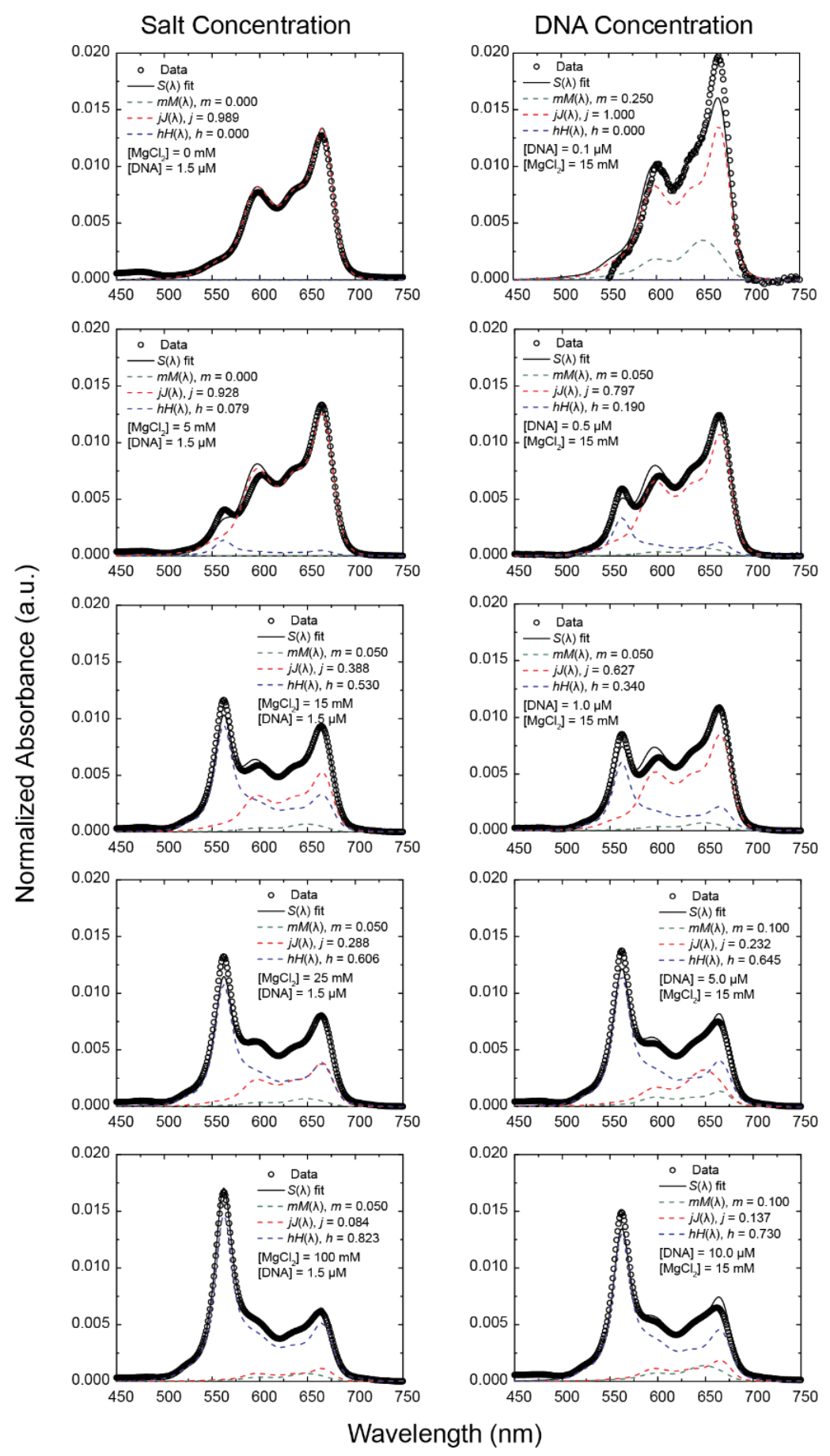

Figure 2.17. UV-Vis absorbance scans (normalized by area) showing spectral changes as a function of salt ( $\mathrm{MgCl}_{2}$, left panels) and DNA (right panels) concentrations. Each scan is fit to Eq. (2.75) to give relative percentages of each individual aggregate state: $\boldsymbol{m}$ (monomer), $\boldsymbol{j}$ (J-dimer), and $\boldsymbol{h}$ (H-tetramer).

To accurately describe the DNA concentration dependence of the aggregation state transition, we have the following two theoretical equations: 


$$
\frac{[J]}{[J]+2[H]}=\frac{\sqrt{1+8 K_{e q}[D N A]}-1}{4 K_{e q}[D N A]}
$$

and

$$
\frac{2[H]}{[J]+2[H]}=1-\frac{\sqrt{1+8 K_{e q}[D N A]}-1}{4 K_{e q}[D N A]}
$$

where $[J],[H]$, and $[D N A]$ represent the J-dimer, H-tetramer, and total DNA concentrations, respectively, and $K_{e q}$ is the equilibrium constant for the transition reaction. The $[J]$ and $[H]$ values were determined using the linear combination fitting procedure described above (i.e., $j$ and $h$ ), and found to be those indicated in the right hand panel of Figure 2.17. To see the full derivation of these equations, please refer to section 2.3. The data shown in Figure $4 \mathrm{~d}$ (samples held at $15 \mathrm{mM} \mathrm{MgCl} 2$ and room temperature with variations in DNA concentration) were fit to Eqs. (2.42) and (2.43) using a least-squares fitting procedure. From the fit, the equilibrium constant, $K_{e q}$, was found to be $51 \mathrm{M}^{-1}$ (Table 2.14). Note that the two fits provided equivalent results, as the data are not independently measured, such that Eq. (2.43) is merely one minus Eq. (2.42).

\subsection{Experimental Methods}

\subsubsection{Preparation of DNA-Templated Dye Aggregate Constructs}

Short 26 bp DNA oligomers internally functionalized with Cy5 dyes were purchased lyophilized and purified via high-performance liquid chromatography (HPLC) from Bio-Synthesis, Inc. (Lewisville, TX, USA) and Integrated DNA Technologies, Inc. (Coralville, IA, USA). Oligomers were rehydrated with ultrapure water (Barnstead Nanopure, Thermo Scientific) to create a $100 \mu \mathrm{M}$ stock solution that was used without further purification. The Cy5 dye structure and DNA sequences are provided in section 2.7.1. DNA duplexes were prepared by combining equimolar amounts of complementary 
oligomers in a $1 \times$ TAE (40 mM tris(hydroxymethyl)aminomethane, $20 \mathrm{mM}$ acetic acid, 1 $\mathrm{mM}$ ethylenediaminetetraacetic acid) buffer solution ( $\mathrm{pH}$ 8.0) with 0-100 $\mathrm{mM}$ added magnesium chloride $\left(\mathrm{MgCl}_{2}\right)$ to yield a final DNA concentration of $0.1-20 \mu \mathrm{M}$. Resulting solutions were then allowed to hybridize for 24 hours at room temperature. TAE (10x stock solution) and $\mathrm{MgCl}_{2}$ (99\% purity) were purchased from Fisher Scientific and used as received. Aliquots of a $1.375 \mathrm{M}$ stock solution of $\mathrm{MgCl}_{2}$ were added to the diluted (1x) TAE buffer solution to produce solutions with final magnesium $\left(\mathrm{Mg}^{2+}\right)$ concentrations of $0,5,12,15$, and $100 \mathrm{mM} \mathrm{MgCl}_{2}$. The initial salt concentration due to the TAE buffer $\left(<1 \mathrm{mM} \mathrm{Na}^{+}\right.$arising from the EDTA counterion) was found to be negligible compared to the subsequently added salt concentrations.

\subsubsection{Optical Characterization of Dye Aggregates}

All optical characterization was performed at room temperature unless otherwise noted. UV-Vis absorbance spectra were collected using a dual-beam Cary 5000 UV-VisNIR spectrophotometer (Agilent Technologies) by pipetting $150 \mu \mathrm{L}$ of sample solution into a $1 \mathrm{~cm}$ path length sub-micro, self-masking quartz spectrophotometer cell (Starna). Prior to synthesis of dye-DNA constructs, concentrations of ssDNA oligomers were quantified by measuring the UV absorbance at $260 \mathrm{~nm}$ using the Cary 5000 . Static absorbance spectra were taken over a range of wavelengths and normalized by the resulting DNA concentration. All absorption and fluorescence spectra of the Cy5 monomer was conducted using a solution sample of oligomers labeled with a single Cy5 dye hybridized with a complementary non-dye labeled strand in $1 \times$ TAE buffer with 15 $\mathrm{mM}$ added $\mathrm{MgCl}_{2}$. Similarly, J- and $\mathrm{H}$-aggregates were prepared by combining equimolar amounts of a Cy5 labeled oligomer with a second complimentary Cy5 labeled oligomer 
in a $1 \times$ TAE buffer with $0 \mathrm{mM}$ and $155 \mathrm{mM}$ added $\mathrm{MgCl}_{2}$, respectively. See section 2.7.6 for similar experiments conducted with $\mathrm{NaCl}$.

The procedure used to obtain reaction kinetics spectra as a function of time and temperature is illustrated in Figure 2.5. Isothermal reaction kinetics data for both the forward and reverse reactions were collected by recording changes in the H-tetramer absorbance peak at $562 \mathrm{~nm}$ every 0.1 seconds for a series of temperatures $\left(20^{\circ} \mathrm{C}, 22^{\circ} \mathrm{C}\right.$, $25^{\circ} \mathrm{C}$, and $30^{\circ} \mathrm{C}$ ). For the transition reaction where J-dimers at $0 \mathrm{mM}$ added $\mathrm{MgCl}_{2}$ associate to form $\mathrm{H}$-tetramers at a final salt concentration of $93.75 \mathrm{mM} \mathrm{MgCl} 2\left(\mathrm{~J}_{0} \rightarrow \mathrm{H}_{93.75}\right.$, Figure 2.5aError! Reference source not found.), dye-DNA constructs were initially $p$ repared at $20 \mu \mathrm{M}$ dsDNA concentration with no $(0 \mathrm{mM})$ added $\mathrm{MgCl}_{2}$ and allowed to reach thermal and chemical equilibrium. A single small $(10 \mu \mathrm{L})$ aliquot of the stock 20 $\mu \mathrm{M}$ dye DNA solution was then added to a cuvette containing $150 \mu \mathrm{L}$ of $1 \times \mathrm{TAE}$ solution with $100 \mathrm{mM}$ added $\mathrm{MgCl}_{2}$, and the absorption was monitored until equilibrium was reached (50-400 minutes depending upon the temperature). The final salt and DNA concentrations were $93.75 \mathrm{mM} \mathrm{MgCl}_{2}$ and $1.25 \mu \mathrm{M}$ DNA, respectively.

To examine the reaction in which $\mathrm{H}$-tetramers at $100 \mathrm{mM} \mathrm{MgCl} 2$ dissociate into pairs of J-dimers with a final salt concentration of $6.25 \mathrm{mM} \mathrm{MgCl} 2\left(\mathrm{H}_{100} \rightarrow \mathrm{J}_{6.25}\right.$, Figure 2.5b), dye-DNA samples were prepared in a $1 \times$ TAE solution with $100 \mathrm{mM} \mathrm{MgCl}_{2}$ added and left for 24 hours to reach thermal and chemical equilibrium. Upon adding $10 \mu \mathrm{L}$ of the stock $20 \mu \mathrm{M}$ dye-DNA sample into $150 \mu \mathrm{L}$ of $1 \times$ TAE buffer solution with $0 \mathrm{mM}$ added $\mathrm{MgCl}_{2}$, the sample was monitored over 50-400 minutes (depending upon the temperature) until equilibrium was reached. The final DNA concentration was $1.25 \mu \mathrm{M}$ and $6.25 \mathrm{mM}$ added $\mathrm{MgCl}_{2}$ present. 
Static fluorescence spectra of each dye-DNA construct (i.e., monomer, J-dimer, and H-tetramer) were obtained from $250 \mu \mathrm{L}$ of dye-DNA sample solution pipette into a 4 mm path length micro square, open-top special optical glass (SOG) fluorometer cell (Starna). Measurements were taken over a range of fluorescence emission wavelengths within the visible region while exciting the constructs at their respective absorption maxima using a Fluorolog-3 spectrofluorometer (HORIBA Scientific).

\subsubsection{Structural Characterization of DNA-Templated Dye Aggregates}

Polyacrylamide gel-electrophoresis (PAGE) was used to assess the structural differences between the two states (i.e., J-dimer and H-tetramer). Solutions of DNA constructs with carious dye arrangements in TAE buffer at $20 \mu \mathrm{M}$ dsDNA concentrations were mixed in a 5:1 ratio with $6 \times$ New England Biolabs loading buffer (final concentrations in resultant $1 \times$ buffer: $11 \mathrm{mM}$ ethylenediaminetetraacetic acid, $3.3 \mathrm{mM}$ tris-hydrochloric acid, 0.017\% Ficoll@-400), injected into a 12\% PAGE gel, and allowed to run for approximately 90-120 minutes with a 75-150 V applied voltage. The sample and running buffers were composed of a $1 \times$ TAE solution with either $0 \mathrm{mM}$ or $100 \mathrm{mM}$ added $\mathrm{MgCl}_{2}$. Completed PAGE gels were placed on a phosphor imaging plate and imaged while being briefly illuminated with a $254 \mathrm{~nm}$ UV light source using a multiplexed gel imaging and documentation system (Fluorchem Q, ProteinSimple). Wellformed dye-DNA construct bands were identified, cut from the gels with a razor blade, extracted into a TAE buffer solution, and optically characterized using a Cary 5000 spectrophotometer. Extraction was conducted by placing select cut gel pieces into a vial of $1 \times$ TAE buffer containing the desired concentration of $\mathrm{MgCl}_{2}$. The DNA structures were then allowed 24-48 hours to diffuse out of the gel and into the surrounding buffer 
solution. The sample buffer solution was then extracted from the vial and any remaining gel fragments removed by centrifuging the sample at 1000 relative centrifugal force (rcf) for 10 minutes. The UV-Vis spectrum collected from structures that were identified via PAGE as exclusively $\mathrm{J}$-dimer constructs (at $0 \mathrm{mM} \mathrm{MgCl}$ ) was used as a pure $\mathrm{J}$-aggregate control (see section 2.7.8). A similar procedure was followed for the structures identified as H-tetramers (at $100 \mathrm{mM} \mathrm{MgCl}_{2}$ ) to obtain a pure $\mathrm{H}$-tetramer control spectrum. 


\section{REFERENCES}

[1] Fassioli, F.; Dinshaw, R.; Arpin, P. C.; Scholes, G. D., Photosynthetic Light Harvesting: Excitons and Coherence. Journal of Royal Soceity Interface 2014, 11 (92).

[2] Streltsov, A.; Singh, U.; Dhar, H. S.; Bera, M. N.; Adesso, G., Measuring Quantum Coherence with Entanglement. Physical Reviews Letters 2015, 115 (2).

[3] Baumgratz, T.; Cramer, M.; Plenio, M. B., Quantifying Coherence. Physical Review Letters 2014, 113 (14).

[4] Collini, E., Spectroscopic Signatures of Quantum-Coherent Energy Transfer. Chemical Society Reviews 2013, 42 (12), 4932-4947.

[5] Blancafort, L.; Voityuk, A. A., Exciton Delocalization, Charge Transfer, and Electronic Coupling for Singlet Excitation Energy Transfer Between Stacked Nucleobases in DNA: An MS-CASPT2 Study. Journal of Chemical Physics 2014, 140 (9).

[6] Briggs, J. S.; Eisfeld, A., Equivalence of Quantum and Classical Coherence in Electronic Energy Transfer. Physical Reviews E 2011, 83 (5).

[7] Briggs, J. S.; Eisfeld, A., Coherent Quantum States from Classical Oscillator Amplitudes. Physical Reviews A 2012, 85 (5).

[8] Briggs, J. S.; Eisfeld, A., Quantum Dynamics Simulation with Classical Oscillators. Physical Reviews A 2013, 88 (6).

[9] Eisfeld, A.; Briggs, J. S., The J-Band of Organic Dyes: Lineshape and Coherence Length. Chemical Physics 2002, 281 (1), 61-70.

[10] Eisfeld, A.; Briggs, J. S., Absorption Spectra of Quantum Aggregates Interacting via Long-Range Forces. Physical Review Letters 2006, 96 (11).

[11] Eisfeld, A.; Schulz, G.; Briggs, J., The Influence of Geometry on the Vibronic Spectra of Quantum Aggregates. Journal of Luminescence 2011, 131 (12), 2555-2564. 
[12] Asanuma, H.; Fujii, T.; Kato, T.; Kashida, H., Coherent Interactions of Dyes Assembled on DNA. J. Photochemistry and Photobiology C 2012, 13 (2), 124-135.

[13] Engel, G. S., Quantum Coherence in Photosynthesis. Procedia Chemistry 2011, 3, 222-231.

[14] Fidler, A. F.; Caram, J. R.; Hayes, D.; Engel, G. S., Towards a Coherent Picture of Excitonic Coherence in the Fenna-Matthews-Olson Complex. Journal of Physics B 2012, 45(15).

[15] Saikin, S. K.; Eisfeld, A.; Valleau, S.; Aspuru-Guzik, A., Photonics Meets Excitonics: Natural and Artificial Molecular Aggregates. Nanophotonics 2013, 2 (1), 21-38.

[16] DiVincenzo, D. P.; Loss, D., Quantum Computers and Quantum Coherence. Journal of Magnetism and Magnetic Materialsagn 1999, 200 (1-3), 202-218.

[17] Unruh, W. G., Maintaing Coherence in Quantum Computers. Physical Review A 1995, $51(2), 992-997$.

[18] Davydov, A. S., Theory of Absorption Spectra of Molecular Crystals. Institute of Physics, Academy of Sciences of Ukrainian SSR: Kyiv, Ukraine, 1948; Vol. 18.

[19] Davydov, A. S., The Theory of Molecular Excitons. Soviet Physics 1964, 82 (3-4), 145-178.

[20] Kasha, M., Energy Transfer Mechanisms and Molecular Exciton Model for Molecular Aggregates. Radiation Research 1963, 20 (1), 55-\&.

[21] Kasha, M., The Exciton Model in Molecular Spectroscopy. Pure and Applied Chemistry 1965, 11 (3-4), 371.

[22] Eisfeld, A.; Briggs, J. S., The J- and H-Bands of Organic Dye Aggregates. Chemical Physics 2006, 324 (2-3), 376-384.

[23] Eisfeld, A.; Briggs, J. S., The Shape of the J-Band of Pseudoisocyanine. Chemical Physics Letters 2007, 446 (4-6), 354-358.

[24] Gross, M.; Haroche, S., Super-Radiance - An Essay on the Theory of Collective Spontaneous Emission. Physical Reports 1982, 93 (5), 301-396.

[25] Dicke, R. H., Coherence in Spontaneous Radiation Processes. Physical Reviews 1954, 93 (1), 99-110. 
[26] Spano, F. C.; Kuklinski, J. R.; Mukamel, S., Temperature-Dependent Superradiant Decay of Excitons in Small Aggregates. Physical Review Letters 1990, 65 (2), 211214.

[27] Scholes, G. D.; Ghiggino, K. P.; Oliver, A. M.; Paddonrow, M. N., Through-Space and Through-Bond Effects on Exciton Interactions in Rigidly Linked Dinaphthyl Molecules. Journal of the American Chemical Society 1993, 115 (10), 4345-4349.

[28] Pajusalu, M.; Raetsep, M.; Trinkunas, G.; Freiberg, A., Davydov Splitting of Excitons in Cyclic Bacteriochlorophyll a Nanoaggregates of Bacterial Light-Harvesting Complexes between 4.5 and 263 K. Chemphyschem 2011, 12 (3), 634-644.

[29] Zimanyi, E. N.; Silbey, R. J., Theoretical description of quantum effects in multichromophoric aggregates. Philosophical Transactions of the Royal Society A 2012, 370 (1972), 3620-3637.

[30] Wuerthner, F.; Kaiser, T. E.; Saha-Moeller, C. R., J-Aggregates: From Serendipitous Discovery to Supramolecular Engineering of Functional Dye Materials. Angewandte Chemie - International Edition 2011, 50 (15), 3376-3410.

[31] Wang, M. M.; Silva, G. L.; Armitage, B. A., DNA-Templated Formation of a Helical Cyanine Dye J-aggregate. Journal of the American Chemcal Society 2000, 122 (41), 9977-9986.

[32] von Berlepsch, H.; Bottcher, C., Supramolecular Structure of TTBC J-Aggregates in Solution and on Surface. Langmuir 2013, 29 (16), 4948-4958.

[33] Stiel, H.; Teuchner, K.; Becker, W.; Freyer, W.; Dahne, S., Fluorescence Lifetime Studies of Pseudoisocyanin J-Aggregates in the Subnanosecond Range. Journal of Molecular Structure 1984, 114, 351-354.

[34] Markova, L. I.; Malinovskii, V. L.; Patsenker, L. D.; Haner, R., J- vs. H-Type Assembly: Pentamethine Cyanine (Cy5) as a Near-IR Chiroptical Reporter. Chemical Communications 2013, 49 (46), 5298-5300.

[35] Li, Z. a.; Mukhopadhyay, S.; Jang, S.-H.; Bredas, J.-L.; Jen, A. K. Y., Supramolecular Assembly of Complementary Cyanine Salt J-Aggregates. Journal of the American Chemical Society 2015, 137 (37), 11920-11923.

[36] Jones, R. M.; Bergstedt, T. S.; Buscher, C. T.; McBranch, D.; Whitten, D., Superquenching and Its Applications in J-Aggregated Cyanine Polymers. Langmuir 2001, 17 (9), 2568-2571. 
[37] Iwaura, R.; Ohnishi-Kameyama, M.; Iizawa, T., Construction of Helical J-Aggregates Self-Assembled from a Thymidylic Acid Appended Anthracene Dye and DNA as a Template. Chemistry - A European Journal 2009, 15 (15), 3729-3735.

[38] Herrera, F.; Peropadre, B.; Pachon, L. A.; Saikin, S. K.; Aspuru-Guzik, A., Quantum Nonlinear Optics with Polar J-Aggregates in Microcavities. Journal of Physical Chemistry Letters 2014, 5 (21), 3708-3715.

[39] Gadde, S.; Batchelor, E. K.; Weiss, J. P.; Ling, Y. H.; Kaifer, A. E., Control of H- and J-Aggregate Formation via Host-Guest Complexation using Cucurbituril Hosts. Journal of the American Chemical Society 2008, 130 (50), 17114-17119.

[40] Fidder, H.; Knoester, J.; Wiersma, D. A., Superradiant Emission and Optical Dephasing in J-Aggregates. Chemical Physics Letters 1990, 171 (5-6), 529-536.

[41] Ruedas-Rama, M. J.; Orte, A.; Martin-Domingo, M. C.; Castello, F.; Talavera, E. M.; Alvarez-Pez, J. M., Interaction of YOYO-3 with Different DNA Templates to Form H-Aggregates. Journal of Physical Chemistry B 2014, 118 (23), 6098-6106.

[42] Ruedas-Rama, M. J.; Alvarez-Pez, J. M.; Orte, A., Formation of Stable BOBO-3 HAggregate Complexes Hinders DNA Hybridization. Journal of Physical Chemistry B 2010, 114 (27), 9063-9071.

[43] Ikeda, S.; Okamoto, A., Hybridization-Sensitive On-Off DNA Probe: Application of the Exciton Coupling Effect to Effective Fluorescence Quenching. Chemistry - An Asian Journal 2008, 3 (6), 958-968.

[44] Asanuma, H.; Shirasuka, K.; Takarada, T.; Kashida, H.; Komiyama, M., DNA-Dye Conjugates for Controllable $\mathrm{H}^{*}$ Aggregation. Journal of the American Chemical Society 2003, 125 (8), 2217-2223.

[45] Ikeda, S.; Kubota, T.; Kino, K.; Okamoto, A., Sequence Dependence of Fluorescence Emission and Quenching of Doubly Thiazole Orange Labeled DNA: Effective Design of a Hybridization-Sensitive Probe. Bioconjugate Chemistry 2008, 19 (8), 1719-1725.

[46] Armitage, B. A., Cyanine Dye-DNA Interactions: Intercalation, Groove Binding, and Aggregation. Top Curr. Chem. 2005, 253, 55-76.

[47] Hannah, K. C.; Armitage, B. A., DNA-Templated Assembly of Helical Cyanine Dye Aggregates: A Supramolecular Chain Polymerization. Accounts of Chemical Research 2004, 37 (11), 845-853. 
[48] Teo, Y. N.; Kool, E. T., DNA-Multichromophore Systems. Chemical Reviews 2012, $112(7), 4221-4245$.

[49] Knapp, E. W., Lineshapes of Molecular Aggregates - Exchange Narrowing and Intersite Correlation. Chemical Physics 1984, 85 (1), 73-82.

[50] Whittaker, D. M.; Kinsler, P.; Fisher, T. A.; Skolnick, M. S.; Armitage, A.; Afshar, A. M.; Sturge, M. D.; Roberts, J. S., Motional Narrowing in Semiconductor Microcavities. Physical Review Letters 1996, 77 (23), 4792-4795.

[51] Bonifacio, R.; Lugiato, L. A., Cooperative Radiation Processes in 2-Level Systems Superfluorescence. Phys. Rev. A 1975, 11 (5), 1507-1521.

[52] Bonifacio, R.; Lugiato, L. A., Cooperative Radiation Processes in 2-Level Systems Superfluorescence .2. Physical Reviews A 1975, 12 (2), 587-598.

[53] Marcus, R. J.; Haugen, G. R., Resonance Fluorescence in Chlorophyll a Solutions. Photochemistry and Photobiology 1965, 4, 183-192.

[54] Herz, A. H., Aggregation of Sensitizing Dyes in Solution and Their Adsorption onto Silver Halides. Advanced Colloid Interface Science 1977, 8 (4), 237-298.

[55] Mobius, D., Scheibe Aggregates. Advanced Materials 1995, 7 (5), 437-444.

[56] Malinovskii, V. L.; Wenger, D.; Haner, R., Nucleic Acid-Guided Assembly of Aromatic Chromophores. Chemical Society Reviews 2010, 39 (2), 410-422.

[57] Jelley, E. E., Spectral Absorption and Fluorescence of Dyes in the Molecular State. Nature 1936, 138, 1009-1010.

[58] Jelley, E. E., Molecular, Nematic and Crystal States of I: I-Diethyl-Cyanine Chloride. Nature 1937, 139 (3519), 631-632.

[59] Scheibe, G.; Kandler, L.; Ecker, H., Polymerisation and Polymere Adsorption as a Cause of Novel Absorption Bands of Organic Pigments. Naturwissenschaften 1937, $25,75-75$.

[60] Scheibe, G.; Mareis, A.; Ecker, H., The reverible polymerisation as a cause of unusual absorption bands III. Naturwissenschaften 1937, 25, 474-475.

[61] Scheibe, G., Über die Veränderlichkeit der Absorptionsspektren in Lösungen und die Nebenvalenzen als ihre Ursache. Angewandte Chemie 1937, 50.11, 212-219. 
[62] Fujii, T.; Kashida, H.; Asanuma, H., Analysis of Coherent Heteroclustering of Different Dyes by Use of Threoninol Nucleotides for Comparison with the Molecular Exciton Theory. Chemistry - A European Journal 2009, 15 (39), 10092-10102.

[63] Conley, N. R.; Pomerantz, A. K.; Wang, H.; Twieg, R. J.; Moerner, W. E., Bulk and Single-Molecule Characterization of an Improved Molecular Beacon Utilizing HDimer Excitonic Behavior. Journal of Physical Chemistry B 2007, 111 (28), 79297931.

[64] Ogawa, M.; Kosaka, N.; Choyke, P. L.; Kobayashi, H., H-Type Dimer Formation of Fluorophores: A Mechanism for Activatable, in Vivo Optical Molecular Imaging. ACS Chemical Biology 2009, 4 (7), 535-546.

[65] Scholes, G. D., Quantum Biology Coherence in photosynthesis. Nature Physics 2011, 7 (6), 448-449.

[66] Nalbach, P.; Thorwart, M., Quantum Coherence and Entanglement in Photosynthetic Light-Harvesting Complexes. Semiconductors and Semimetals, Weber, E. R.; Thorwart, M.; Wurfel, U., 2010; 83, 39-75.

[67] Ishizaki, A.; Fleming, G. R., Quantum Coherence in Photosynthetic Light Harvesting. Annual Reviews of Condenensed Matieral Physics, Langer, J. S., 2012; 3, 333-361.

[68] Engel, G. S.; Calhoun, T. R.; Read, E. L.; Ahn, T.-K.; Mancal, T.; Cheng, Y.-C.; Blankenship, R. E.; Fleming, G. R., Evidence for Wavelike Energy Transfer Through Quantum Coherence in Photosynthetic Systems. Nature 2007, 446 (7137), 782-786.

[69] Arndt, M.; Juffmann, T.; Vedral, V., Quantum Physics Meets Biology. HFSP Journal 2009, 3 (6), 386-400.

[70] Goodsell, D. S., Biomolecules and Nanotechnology. American Science 2000, 88 (3), 230-237.

[71] Nafisi, S.; Saboury, A. A.; Keramat, N.; Neault, J. F.; Tajmir-Riahi, H. A., Stability and Structural Features of DNA Intercalation with Ethidium Bromide, Acridine Orange and Methylene Blue. Journal of Moelcuar Structure 2007, 827 (1-3), 35-43.

[72] Kelley, S. O.; Barton, J. K., DNA-Mediated Electron Transfer from a Modified Base to Ethidium: Pi-Stacking as a Modulator of Reactivity. Chemical Biology 1998, 5 (8), 413-425.

[73] Haner, R.; Samain, F.; Malinovskii, V. L., DNA-Assisted Self-Assembly of Pyrene Foldamers. Chemistry - A European Journal 2009, 15 (23), 5701-5708. 
[74] Markova, L. I.; Malinovskii, V. L.; Patsenker, L. D.; Haner, R., Synthesis and Properties of Squaraine-Modified DNA. Organic \& Biomolecular Chemistry 2012, 10 (45), 8944-8947.

[75] Kashida, H.; Asanuma, H., Preparation of Supramolecular Chromophoric Assemblies Using a DNA Duplex. Physical Chemistry Chemical Physics 2012, 14 (20), 71967204.

[76] Kashida, H.; Tanaka, M.; Baba, S.; Sakamoto, T.; Kawai, G.; Asanuma, H.; Komiyama, M., Covalent Incorporation of Methyl Red Dyes into Double-Stranded DNA for Their Ordered Clustering. Chemistry - A European Journal 2006, 12 (3), 777-784.

[77] Conley, N. R.; Biteen, J. S.; Moerner, W. E., Cy3-Cy5 Covalent Heterodimers for Single-Molecule Photoswitching. Journal of Physical Chemistry B 2008, 112 (38), 11878-11880.

[78] Zwillinger, D., CRC Standard Mathematical Tables and Formulae. 31st ed.; CRC Press: 2002.

[79] Nygren, J.; Svanvik, N.; Kubista, M., The Interactions Between the Fluorescent Dye Thiazole Orange and DNA. Biopolymers 1998, 46 (1), 39-51.

[80] Kumar, C. V.; Turner, R. S.; Asuncion, E. H., Groove Binding of a Styrylcyanine Dye to the DNA Double Helix - The Salt Effect. Journal of Photochemistry and Photobiology A 1993, 74 (2-3), 231-238.

[81] Mooi, S. M.; Heyne, B., Size Does Matter: How To Control Organization of Organic Dyes in Aqueous Environment Using Specific Ion Effects. Langmuir 2012, 28 (48), 16524-16530.

[82] Mooi, S. M.; Keller, S. N.; Heyne, B., Forcing Aggregation of Cyanine Dyes with Salts: A Fine Line between Dimers and Higher Ordered Aggregates. Langmuir 2014, 30 (32), 9654-9662.

[83] von Berlepsch, H.; Boettcher, C., H-Aggregates of an Indocyanine Cy5 Dye: Transition from Strong to Weak Molecular Coupling. Journal of Physical Chemistry B 2015, 119 (35), 11900-11909.

[84] Kunzler, J.; Samha, L.; Zhang, R.; Samha, H., Investigation of the Effect of Concentration on Molecular Aggregation of Cyanine Dyes in Aqueous Solutions. American Journal of Undergraduate Research. 2011, 9 (4), 1-4. 
[85] Chakraborty, S.; Bhattacharjee, D.; Soda, H.; Tominaga, M.; Suzuki, Y.; Kawamata, J.; Hussain, S. A., Temperature and Concentration Dependence of J-Aggregate of a Cyanine Dye in a Laponite Film Fabricated by Langmuir-Blodgett Technique. Applied Clay Science 2015, 104, 245-251.

[86] Huang, Z. X.; Ji, D. M.; Wang, S. F.; Xia, A. D.; Koberling, F.; Patting, M.; Erdmann, R., Spectral Identification of Specific Photophysics of Cy5 by Means of Ensemble and Single Molecule Measurements. Journal of Physical Chemistry A 2006, 110 (1), 4550 .

[87] Berova, N.; Nakanishi, K., Circular Dichroism: Principles and Applications. John Wiley \& Sons: 2000.

[88] Harada, N.; Nakanish.K, Exciton Chirality Method and Its Application to Configurational and Conformational Studies of Natural Products. Accounts of Chemical Research 1972, 5 (8), 257.

[89] West, W.; Pearce, S., The Dimeric State of Cyanine Dyes. Journal of Physical Chemistry 1964, 69(6), 1894-1903.

[90] Schelly, Z. A.; Harward, D. J.; Hemmes, P.; Eyring, E. M., Bonding in Dye Aggregates - Energetics of Dimerization of Aqueous Cobalt(II)-4,4',4",4"'Tetrasulfophthalocyanine Ion. Journal of Physical Chemistry 1970, 74 (16), 3040.

[91] Kallenbach, N. R.; Ma, R. I.; Seeman, N. C., An Immobile Nucleic-Acid Junction Constructed from Oligonucleotides. Nature 1983, 305 (5937), 829-831.

[92] Mooi, S. M.; Heyne, B. Size Does Matter: How To Control Organization of Organic Dyes in Aqueous Environment Using Specific Ion Effects. Langmuir 2012, 28, 16524-16530.

[93] Kunzler, J.; Samha, L.; Zhang, R.; Samha, H. Investigation of the Effect of Concentration on Molecular Aggregation of Cyanine Dyes in Aqueous Solutions. Am. J. Undergrad. Res. 2011, 9, 1-4.

[94] Nygren, J.; Svanvik, N.; Kubista, M. The Interactions Between the Fluorescent Dye Thiazole Orange and DNA. Biopolymers 1998, 46, 39-51.

[95] Kumar, C. V.; Turner, R. S.; Asuncion, E. H. Groove Binding of a Styrylcyanine Dye to the DNA Double Helix - The Salt Effect. J. Photochem. Photobiol., A 1993, 74, 231-238. 
[96] von Berlepsch, H.; Boettcher, C. H-Aggregates of an Indocyanine Cy5 Dye: Transition from Strong to Weak Molecular Coupling. J. Phys. Chem. B 2015, 119, 11900-11909.

[97] Chakraborty, S.; Bhattacharjee, D.; Soda, H.; Tominaga, M.; Suzuki, Y.; Kawamata, J.; Hussain, S. A. Temperature and Concentration Dependence of J-Aggregate of a Cyanine Dye in a Laponite Film Fabricated by Langmuir-Blodgett Technique. Appl. Clay Sci. 2015, 104, 245-251.

[98] Spano, F. C. The Spectral Signatures of Frenkel Polarons in Hand J-Aggregates. Acc. Chem. Res. 2010, 43, 429-439. 


\section{CHAPTER THREE: LARGE DAVYDOV SPLITTING AND STRONG FLUORESCENCE SUPPRESSION: AN INVESTIGATION OF EXCITON DELOCALIATION IN DNA-TEMPLATED HOLLIDAY JUNCTION DYE AGGREGATES}

This chapter is published by the American Chemical Society in Journal of Physical Chemistry $A$ and should be referenced appropriately.

\section{Reference:}

Cannon, B. L.; Kellis, D. L.; Patten, L. K.; Davis, P. H.; Lee, J.; Graugnard, E.; Yurke, B;

Knowlton, W. B., Large Davydov Splitting and Strong Fluorescence Suppression: An Investigation of Exciton Delocalization in DNA-Templated Holliday Junction Dye Aggregates, Journal of Physical Chemistry A 2017, 122 (8), 2086-2095.

Reproduced/modified by permission of the American Chemical Society.

*This chapter includes modifications from the originally published version. 
Large Davydov Splitting and Strong Fluorescence Suppression: An Investigation of Exciton Delocalization in DNA-Templated Holliday Junction Dye Aggregates

\author{
Brittany L. Cannon ${ }^{\mathrm{a}}$ \\ Lance K. Patten ${ }^{\mathrm{a}}$ \\ Donald L. Kellis ${ }^{\mathrm{a}}$ \\ Elton Graugnard ${ }^{\mathrm{a}}$ \\ Jeunghoon Lee \\ Paul H. Davis ${ }^{\mathrm{a}}$ \\ Bernard Yurke $\mathrm{a}^{\mathrm{ac}}$ \\ William B. Knowlton ${ }^{\mathrm{a}, \mathrm{c}}$
}

\begin{abstract}
${ }^{a}$ School of Materials Science and Engineering, Boise State University
${ }^{\mathrm{b}}$ Department of Chemistry and Biochemistry, Boise State University

${ }^{\mathrm{c}}$ Department of Electrical and Computer Engineering, Boise State University 1920 University Dr., Boise ID 83725 USA.
\end{abstract}




\subsection{Abstract}

Exciton delocalization in dye aggregate systems is a phenomenon that is revealed by spectral features, such as Davydov splitting, J- and H-aggregate behavior, and fluorescence suppression. Using DNA as an architectural template to assemble dye aggregates enables specific control of the aggregate size and dye type, proximal and precise positioning of the dyes within the aggregates, and a method for constructing large, modular two- and three-dimensional arrays. Here, we report on dye aggregates, organized via an immobile Holliday junction DNA template, that exhibit large Davydov splitting of the absorbance spectrum $(125 \mathrm{~nm}, 397.5 \mathrm{meV}), \mathrm{J}$ - and H-aggregate behavior, and near-complete suppression of the fluorescence emission ( $\sim 97.6 \%$ suppression). Due to the unique optical properties of the aggregates, we have demonstrated that our dye aggregate system is a viable candidate as a sensitive absorbance and fluorescence optical reporter. DNA-templated aggregates exhibiting exciton delocalization may find application in optical detection and imaging, light-harvesting, photovoltaics, optical information processing, and quantum computing.

\subsection{Introduction}

Exciton delocalization, first discussed theoretically by Frenkel in the 1930's, and later by Davydov and Kasha, is an optical phenomenon that has been observed in optically-active molecular (i.e., dye) aggregates. ${ }^{1-6}$ Exciton (i.e., electron-hole pairs) delocalization is the spatial extension of excited electronic eigenstates across the aggregate, with the resultant excitonic state described by a coherent linear superposition of excited states. ${ }^{7} 8$ Among the observable optical manifestations of excitonic delocalization are Davydov splitting, ${ }^{2,3} \mathrm{~J}$ - and $\mathrm{H}$-aggregate behavior, 4, 5, 7, 9, 10 motional 
narrowing, ${ }^{11-13}$ Dicke superradiance, resonance fluorescence, ${ }^{14}$ fluorescence quenching, ${ }^{15-}$ ${ }^{19}$ and excitonically-coupled circular dichroism (EC-CD). ${ }^{20,21}$ The optical behavior of such aggregates is largely dictated by the stacking arrangement of the closely spaced dye molecules ( $2 \mathrm{~nm}$ or less apart). A single dye, termed a monomer, has a single one-exciton excited energy state, which is the lowest lying excited singlet state. A transition between this excited state and the ground state is allowed (Figure 3.1a). A two-dye aggregate system, termed a dimer, has two one-exciton states in which the degeneracy of the two excited states has been broken by the coupling between the transition dipoles, thus resulting in two energy eigenstates: $\mathrm{E}^{+}$and $\mathrm{E}^{-}$. The exciton is delocalized between the two dyes in these eigenstates. Transitions strengths between these states and the ground state, $\mathrm{E}_{0}$, are strongly dependent on geometry. In the simplest geometric configurations, the two dyes can stack such that the transition dipoles are oriented parallel (H-dimer, Figure 3.1b), head-to-tail (J-dimer, Figure 1c), or at an intermediate configuration (oblique, Figure 3.1d). For these three possible cases, the allowed transitions are indicated by solid black arrows, while the forbidden transitions are indicated by dashed arrows in Figure 3.1. H-type aggregates exhibit a blue-shifted absorbance peak relative to the monomer, significantly reduced fluorescence emission intensity due to an optically forbidden energy transition from the lower energy exciton excited state (E-), and a large Stokes shift. ${ }^{9}$ 15-17, ${ }^{22-30}$ Conversely, J-type aggregates, discovered by Jelley ${ }^{31,32}$ and Scheibe, ${ }^{33-35}$ exhibit a red-shift in the absorbance relative to the monomer since the only allowed optical transition is to the E- state, which consequently produce a nearly resonant fluorescence emission (i.e., small Stokes shift) with a sharp, high-intensity emission peak..$^{7,9,12, ~ 14, ~ 27, ~ 36-~}$ ${ }^{42}$ Oblique aggregates display band (i.e., Davydov) splitting of the absorbance spectrum 
in which optical transitions to both $\mathrm{E}^{-}$and $\mathrm{E}^{+}$are allowed and reflect a mix of $\mathrm{H}-$ and $\mathrm{J}-$ aggregate optical properties. ${ }^{2,3,27,43,44}$
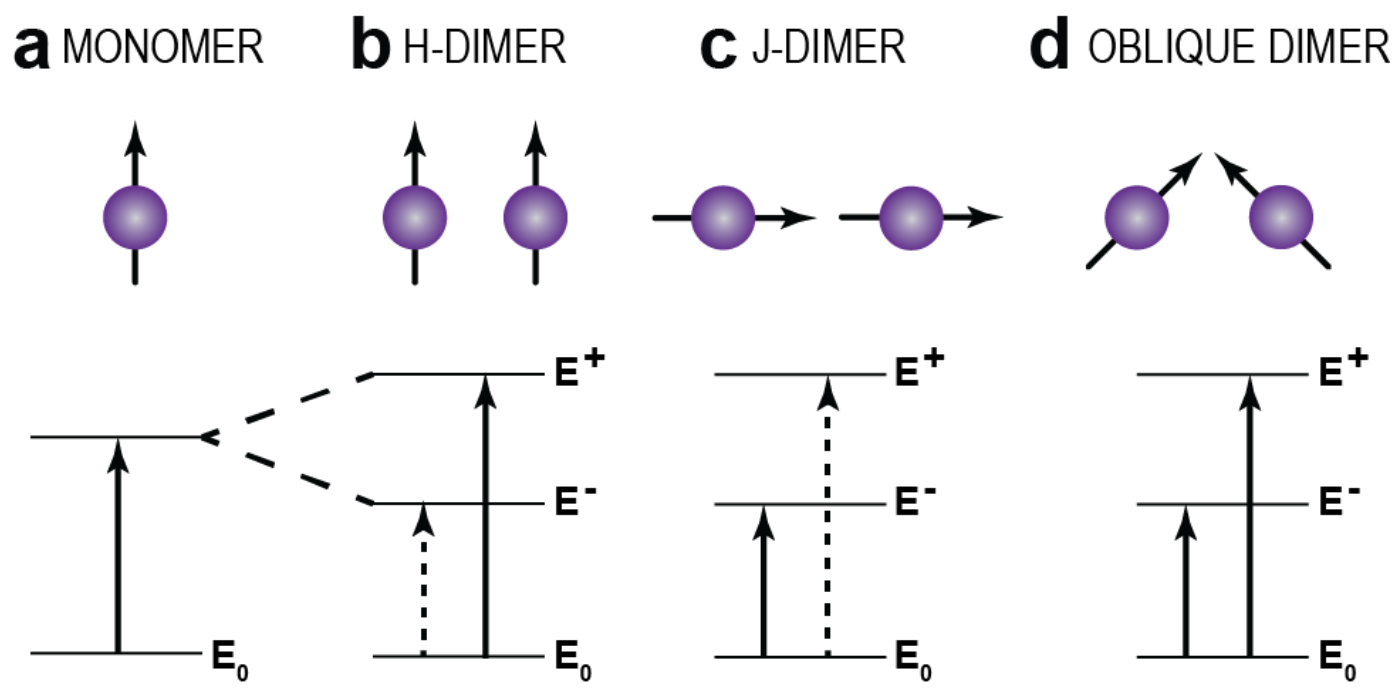

Figure 3.1. Schematics and energy diagrams for various dye aggregate arrangements: (a) monomer, (b) H-dimer, (c) J-dimer, and (d) oblique dimer. The top schematic shows dye molecules represented as purple spheres, while the dipole orientation is depicted by black arrows. Each energy diagram shows a ground energy state $\left(E_{0}\right)$, the first excited energy state $(E)$ for the monomer, and the excited energy state's split energy levels (higher energy, $E^{+}$and lower energy, $E^{-}$) for dimers due to exciton delocalization. Allowed energy transitions are provided as solid black arrows and forbidden transitions are shown as dashed black arrows. Adapted from Kasha with permission. ${ }^{4}$

The manifestation of excitonic delocalization has been observed in both naturally occurring photosynthetic systems ${ }^{45-47}$ and in synthetic dye-solvent systems. ${ }^{29,39,47-51}$ Photosynthetic systems have evolved such that the molecular dyes are proximally positioned via a protein scaffold. However, using a protein scaffolding architecture in synthetic systems is difficult owing to complex folding mechanisms. The aggregation of dyes in solvents has been found to occur spontaneously and preferred dye stacking arrangements are largely driven by kinetics and thermodynamics, which is problematic for controlling the excitonic delocalization behavior. To assert greater control over the assembly of dyes into aggregates of well-defined structure, we exploit DNA as an 
alternative biomolecular scaffolding material. DNA assembly is governed by simple and predictable design rules due largely to Watson-Crick hybridization. In addition, DNA can be functionalized with molecular dyes through covalent attachment at precisely specified locations anywhere along its length. Thus, covalent attachment of dyes onto a DNA substrate enables (i) proximal and precise positioning of the dye molecules, ${ }^{27,}{ }^{52-58}$ (ii) specific and selective control of dye number and type, ${ }^{24,52,54,59}$ and (iii) the self-assembly of large two- and three-dimensional arrays. ${ }^{60-62}$

Capitalizing on the modularity enabled by DNA self-assembly allows larger, more complex arrays and networks of precisely controlled dye aggregates (i.e., dye assemblies) to be realized. Though there have been many studies that demonstrate exciton delocalization in DNA-templated dye aggregation within linear duplex structures, ${ }^{17,18,22-25,27,52-59,63-66}$ only a few studies have exploited DNA constructs of more complex geometry. Most of these have been limited to studies employing threearmed junctions for dye assembly, which have been constructed to explore lightharvesting complexes and excimer behavior. ${ }^{52,67}$ In our recent work, we were able to facilitate and control the transition of Cy5 dimers templated within a linear DNA duplex structure to a Cy5 tetramer in a mobile 4-arm junction (i.e., 4AJ) structure, thereby inducing a change in exciton delocalization from J-type to H-type behavior. ${ }^{68}$ The transition was controlled by varying salt and/or DNA concentrations. Here, we exploit an immobile 4AJ (i.e., Holliday junction) $)^{69-70}$ to explore exciton delocalization in multimers, including dimers, trimers, and tetramers in order to better control the effects of exciton delocalization such as Davydov splitting and J- and H-type behavior at ambient temperatures. The excitonic delocalization between the four $\mathrm{Cy} 5$ dyes (i.e., tetramer) 
located within the core of a 4AJ exhibits an unusually large Davydov splitting (125 nm, $397.5 \mathrm{meV}$ ) that results in a striking color change of the solution, excitonically-coupled circular dichroism (EC-CD) signals, and near-complete quenching (i.e., strong suppression) of the fluorescence emission. To obtain further insight into the 4AJ-guided assembly of excitonically-coupled aggregates, a variety of aggregate types were examined. It was found that increasing the number of dyes within the aggregate core of the $4 \mathrm{AJ}$ substantially increased the energy splitting of the system. Due to its unique optical signatures, this immobile tetramer offers two characterization modes for optical detection that have applications as optical reporters, namely a large shift in absorbance and strong suppression of the fluorescence. The construction, by DNA self-assembly, of dye aggregates exhibiting the strong optical effects reported here may pave the way for novel practical applications of exciton delocalization.

\subsection{Results and Discussion}

\subsubsection{Optical Characterization of 4AJ Templated Dye Aggregates.}

Optical characterization using spectroscopy techniques, including absorbance, fluorescence, and circular dichroism (CD) were performed on a variety of dye-labeled DNA 4AJ structures to identify spectral signatures of exciton delocalization. Although the majority of the data reported here were obtained from samples in a $1 \times$ TAE buffer solution containing $15 \mathrm{mM}$ of $\mathrm{MgCl}_{2}$, we additionally observed absorbance measurements of samples prepared in a $1 \times$ TAE buffer solution containing a variety of salt concentrations: 0 and $100 \mathrm{mM} \mathrm{MgCl}_{2}$ with a constant $1 \mu \mathrm{M}$ DNA concentration and a variety of DNA concentrations: $0.1,1.0$, and $3.0 \mu \mathrm{M}$ with a constant $15 \mathrm{mM}$ salt concentration (see Section 3.7.6) to investigate the salt and DNA concentration 
dependence that might indicate higher order aggregation formation. The absorbance spectra exhibited only a weak dependence on $\mathrm{MgCl}_{2}$ and DNA concentration, indicating that the results obtained at $15 \mathrm{mM} \mathrm{MgCl}_{2}$ concentration are representative of a broad range of salt concentrations and that our $4 \mathrm{AJ}$ structures have little tendency to form higher-ordered aggregates. The minimal dependence on $\mathrm{MgCl}_{2}$ and DNA concentration to drive changes in dye aggregate formation is in contrast to what was found in our previous work with linear DNA duplex constructs that combined to form mobile 4AJ structures. ${ }^{68}$ For spectroscopic studies, all 4AJ structures were purified via polyacrylamide gel-electrophoresis (PAGE). Dye structure and strand information are provided in section 3.7.1. Fluorescence suppression is computed using the area of the emission spectrum; a general equation is given in section 3.7.2.As a control, spectral analysis of the monomer (Figure 3.2a) reveals single absorbance and emission peak maxima at $653 \mathrm{~nm}$ and $666 \mathrm{~nm}$, respectively. Although the monomer absorbance peak maximum is slightly red-shifted from the literature value, the shift is most likely a result of the increased rigidity of the $4 \mathrm{AJ}$ structure, dye-DNA interactions, and surrounding base sequences. ${ }^{71}$ As expected, the dye monomer did not produce a CD signal within the visible range (Figure 3.2b), indicating the absence of molecular chirality and excitonic coupling. Likewise, the CD signals appearing in the UV range at $246 \mathrm{~nm}$ and $276 \mathrm{~nm}$, respectively, result from the expected right-handed macromolecular structure of the $\beta$ DNA helix.

As shown in the schematics in Figure 3.2, the 4AJ structure was used to organize and create four different dye aggregates in addition to the monomer: two dimer configurations, a trimer, and a tetramer. The two-dye (dimer) aggregates are 
distinguished by the location of the dyes, either positioned on adjacent arms (adjacent dimer, Figure 3.2c) or on opposing arms (transverse dimer, Figure 3.2e). A pronounced difference in optical properties was observed between the two dimers. The adjacent dimer shows predominantly J-type aggregate behavior, with an absorbance peak maximum at $662 \mathrm{~nm}$ that is slightly red-shifted relative to the monomer at $653 \mathrm{~nm}$. This red-shift in absorbance relative to the fluorescence peak at $667 \mathrm{~nm}$ yields a small Stokes shift of 5 $\mathrm{nm}$, which is near resonance fluorescence behavior. A second, smaller absorbance peak also appears at $602 \mathrm{~nm}$. The appearance of the two peaks is indicative of a small Davydov splitting of $58 \mathrm{~nm}$ (Table 3.1). The adjacent dimer exhibits a split CD signal (Figure 3.2c) at $600 \mathrm{~nm}$ and $675 \mathrm{~nm}$. The $+/$ - of the CD peaks (read from right to left), signifies right-handed chirality and is anticipated owing to the right-handed nature of the DNA helix. Due to the predominant J-type behavior and presence of Davydov splitting and CD signal, the dyes are most likely arranged head-to-tail with some break in planarity between the molecules, or oblique-like arrangement, that favors J-aggregate stacking. In contrast, the transverse dimer (Figure 3.2e) shows optical behavior that is roughly opposite that of the adjacent dimer. The primary absorbance peak is blue-shifted, with an absorbance maximum at $600 \mathrm{~nm}$ and a much smaller red-shifted absorbance peak (maximum at $636 \mathrm{~nm}$ ) corresponding to an even smaller $36 \mathrm{~nm}$ Davydov splitting. A 68 $\mathrm{nm}$ Stokes shift is observed when comparing the primary absorbance peak to the fluorescence peak at $668 \mathrm{~nm}$, which is much reduced in intensity $(\sim 97.6 \%)$. The blueshifted primary absorbance peak, the large Stokes shift, and the reduced fluorescence intensity are all indicative of predominately $\mathrm{H}$-type aggregate behavior. The small Davydov splitting and a relatively strong -/+ CD signal at $600 \mathrm{~nm}$ and $669 \mathrm{~nm}$, 
respectively, of the transverse dimer indicates left-handed chirality and an oblique stacking arrangement that does not stack in a perfectly parallel or H-type arrangement (Figure 3.2f). Interestingly, the small absorbance peak at $636 \mathrm{~nm}$ corresponds to a split CD signal with peaks at $630 \mathrm{~nm}$ and $669 \mathrm{~nm}$, which implies excitonic coupling between the dyes. The considerably decreased fluorescence emission is indicative of fluorescence suppression due to a forbidden optical transition. 
a

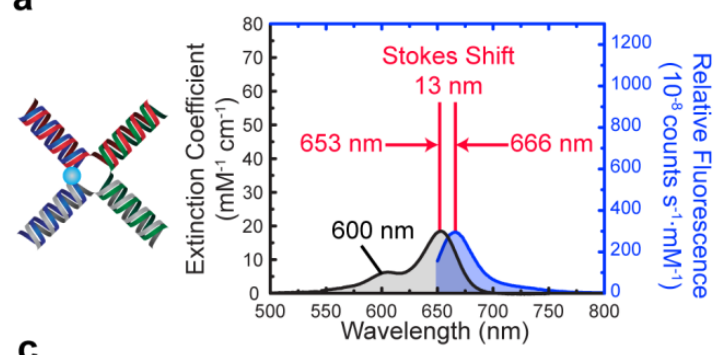

C

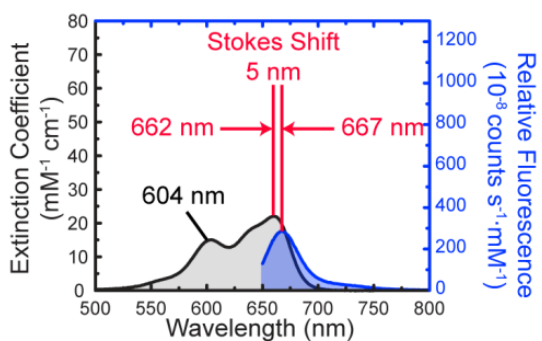

e

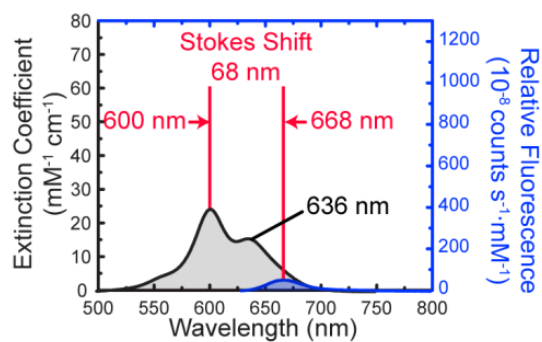

g

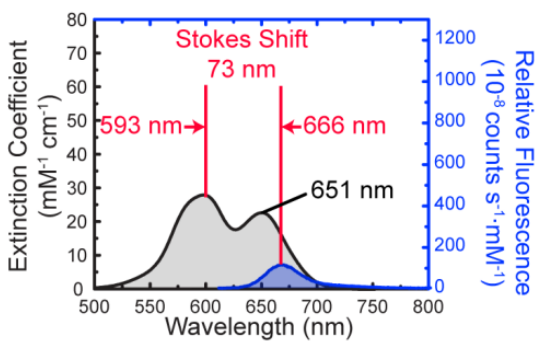

i

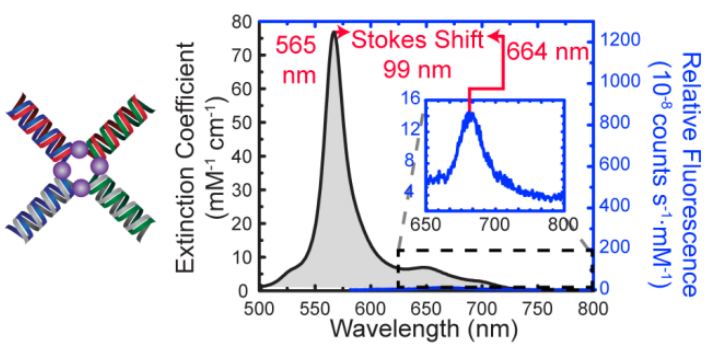

b

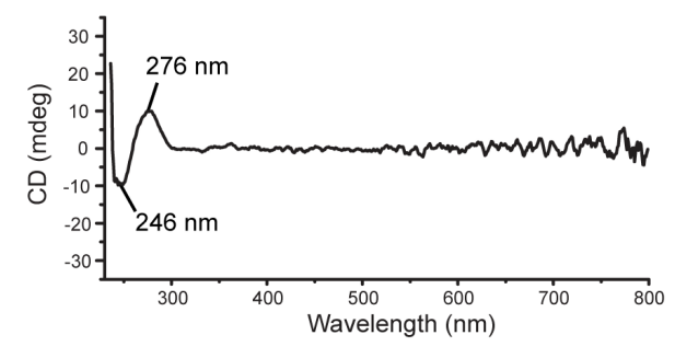

d

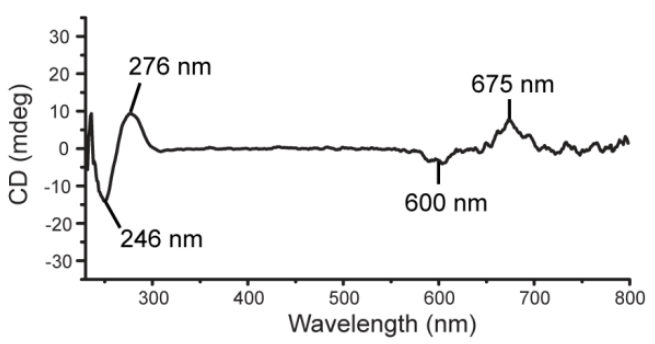

f

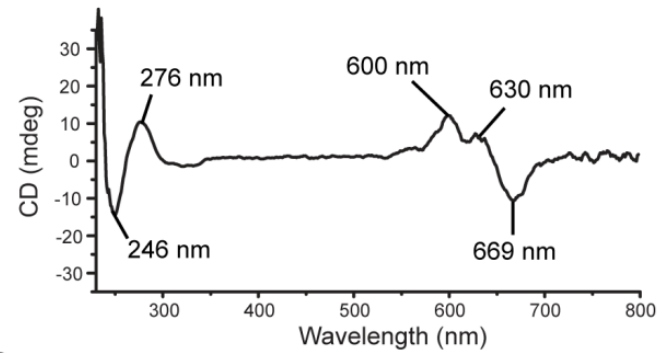

h

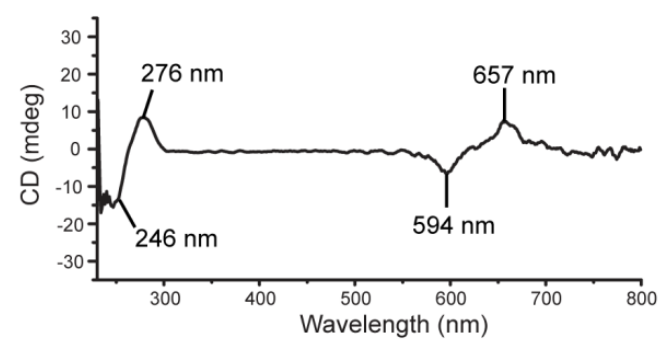

j

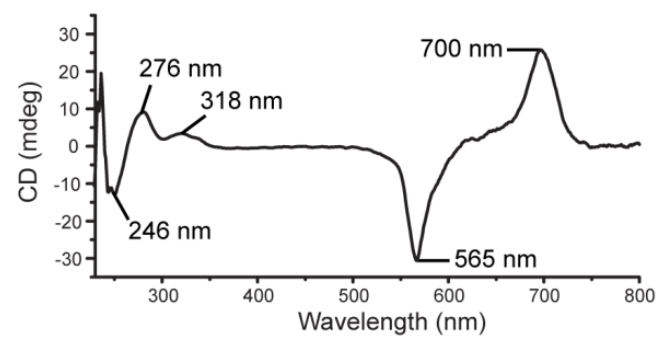

Figure 3.2. Absorbance (black traces in left column), fluorescence (blue traces), and circular dichroism (black traces in right column) spectra corresponding to the 4AJ templated (a,b) monomer, (c,d) adjacent dimer, (e,f) transverse dimer, (g,h) trimer, and (i,j) tetramer dye aggregates. The inset in (i) shows the suppressed fluorescence emission peak at $664 \mathrm{~nm}$ that occurs due to a forbidden energy transfer mechanism. All spectra have been normalized by DNA concentration. The fluorescence studies were performed by monitoring the emission over a range of wavelengths and exciting the aggregates at their respective absorbance maxima. All 
samples were prepared at $10 \mu \mathrm{M}$ DNA concentration in a $1 \times$ TAE buffer solution with $15 \mathrm{mM} \mathrm{MgCl}_{2}$ added, PAGE purified, and normalized by the resulting DNA concentrations $(\sim 0.5-2.5 \mu \mathrm{M})$.

The trimer (Figure 3.2g) shows absorbance and fluorescence properties similar to the transverse dimer. Most notably, the trimer absorbance spectrum shows an absorbance peak at $593 \mathrm{~nm}$ that is blue-shifted from the monomer by $60 \mathrm{~nm}$ and a smaller peak at $651 \mathrm{~nm}$ that is slightly blue-shifted by $2 \mathrm{~nm}$. The absorbance spectrum also reveals broadening of the primary peak by $15 \mathrm{~nm}$ compared to the monomer, as indicated by an increase in the full-width at half-maximum (FWHM) value listed in Table 3.1. A detailed procedure for calculating the FWHM is described in section 3.7.2. The broadening may result from an irresolvable third peak; however, because the CD spectrum (Figure 3.2h) does not exhibit a third peak, the broadening most likely arises from a distribution of trimer dye configurations (i.e., dye positions and orientations). In combination with the blue-shifted absorbance peak, the trimer shows $\sim 85 \%$ suppression of the fluorescence emission intensity (relative to the monomer), indicative of net $\mathrm{H}$-aggregate behavior. Similar to the other aggregate configurations, the trimer has an observed EC-CD signal that, though small, indicates a slightly imperfect stacking arrangement with minor obliqueness. Additionally, the aggregate displays right-handedness, similar to the adjacent dimer.

The tetramer dye aggregate configuration produces the most interesting optical spectra. A large Davydov splitting of $125 \mathrm{~nm}$ (397.5 meV), extensive enough to induce a visible color change in the solution, was observed. See Figure 3.7 for spectral data and peak fitting analysis. To our knowledge, this is the largest reported splitting for DNAtemplated dye aggregates (Figure 3.2i). The Davydov splitting is characterized by a 
significantly blue-shifted intense absorbance peak at $565 \mathrm{~nm}$ and a red-shifted much less intense peak at $690 \mathrm{~nm}$. Though difficult to resolve in the absorption spectrum, the absorbance peak at $690 \mathrm{~nm}$ is further substantiated by a large signal in the CD spectrum (Figure $3.2 \mathrm{j}$ ) at $700 \mathrm{~nm}$. Note that the difference in extinction coefficients of the various dye aggregates result from two key effects: (1) the differences in dye number and (2) exciton delocalization and exciton-vibrational interactions. The tetramer also exhibits strong fluorescence suppression behavior at $664 \mathrm{~nm}$, with a 97.6\% decrease in the fluorescent emission relative to the monomer, as determined by peak area. The large Davydov splitting, strong fluorescence suppression, and $99 \mathrm{~nm}$ Stokes shift (Table 3.1) provide solid evidence of a dye assembly with predominantly H-type stacking. The pronounced $-/+$ CD signal of the tetramer configuration indicates strong exciton coupling between the dyes and reveals that the dyes are oriented predominantly in a parallel manner with some obliqueness that is supported by the absorbance peak at $690 \mathrm{~nm}$. Comparing the optical spectra of the immobile 4AJ-templated tetramer presented here with the mobile 4AJ-templated tetramer observed in our prior study, the most notable difference is in the CD spectrum. Interestingly, the immobile tetramer shows righthandedness (Figure 3.2J), while the mobile tetramer shows left-handedness. ${ }^{68}$ Additionally, an absorbance peak at $665 \mathrm{~nm}$ was observed for the mobile 4AJ-templated tetramer. In contrast, for the immobile tetramer, a very subtle peak was observed for at $690 \mathrm{~nm}$ that was further supported by a strong CD signal, indicating obliqueness within the immobile tetramer. These differences are most likely due to the base-pair stacking of the immobile tetramer locking the aggregate core such that the DNA junction does not undergo restacking. The mobile tetramer undergoes DNA breathing and base-pair 
restacking and was found to partition into two J-dimer pairs that are displaced either horizontally or vertically along the arms, which accounts for the observed $665 \mathrm{~nm}$ absorbance peak. ${ }^{68}$

Table 3.1 Optical excitonic delocalization behavior per 4AJ construct

\begin{tabular}{|c|c|c|c|c|c|}
\hline $\begin{array}{c}\text { Optical } \\
\text { Behavior }\end{array}$ & Monomer & $\begin{array}{c}\text { Adjacent } \\
\text { Dimer }\end{array}$ & $\begin{array}{c}\text { Transverse } \\
\text { Dimer }\end{array}$ & Trimer & Tetramer \\
\hline $\begin{array}{c}\text { Davydov } \\
\text { splitting } \\
(\mathrm{nm} / \mathrm{meV})\end{array}$ & $0 / 0$ & $58 / 179.8$ & $36 / 117.0$ & $58 / 186.3$ & $125 / 397.5$ \\
\hline $\begin{array}{c}\text { Abs FWHM } \\
\text { (nm) }\end{array}$ & 34 & 30 & 24 & 49 & 16 \\
\hline $\begin{array}{c}\text { FL FWHM } \\
(\mathrm{nm})\end{array}$ & 33 & 32 & 34 & 34 & 48 \\
\hline $\begin{array}{c}\text { Stokes shift } \\
(\mathrm{nm})\end{array}$ & 13 & 5 & 68 & 73 & 99 \\
\hline $\begin{array}{c}\% \text { FL } \\
\text { Suppression }\end{array}$ & 0.0 & 53.3 & 89.6 & 84.5 & 97.6 \\
\hline
\end{tabular}

\subsubsection{Theoretical Spectral Modeling of 4AJ Templated Dye Aggregates}

Theoretical modeling of the absorbance and $\mathrm{CD}$ spectra was performed to determine the geometrical stacking configurations of the five dye aggregate configurations since specific dye positioning and orientation beyond the above simple analysis is not readily apparent from visual inspection of experimental spectral data. Following the approach used in our previous work ${ }^{68}$ modeling of the experimental absorbance and CD spectra using a method based on the work of Kühn, Renger, and May, referred to as the Kühn-Renger-May (KRM) model, enables the extraction of the position and orientation of the dyes within each type of aggregate. ${ }^{72}$ The KRM model proceeds by constructing a system Hamiltonian that considers $\mathrm{N}$ dye molecules with arbitrary positions and orientations. Through judicious choice of a truncated Hilbert space, a perturbational treatment of the exciton-vibrational coupling is avoided in the 
diagonalization of the Hamiltonian. Information regarding the computation of the eigenvalues and eigenvectors is described in section 3.7.3. An in-house software simulation tool that incorporates the KRM model was created to fit the absorbance and CD spectra. Smooth theoretical absorbance and CD spectra were obtained by calculating the optical transition rates for each eigenstate and convolving the transition rate line spectra with a Gaussian line shape to approximate the effect of the vibronic continuum on spectral line shape.

In Figure 3.3, theoretical KRM model fits of the absorbance and CD spectra are illustrated by red dashed curves, while the experimental data are shown as black open circles. To maximally constrain the theoretical modeling, the absorbance and CD data were simultaneously fit such that the two dominant peaks of the absorption and CD spectra were both matched and the mean-square difference between the experimental and theoretical absorption and $\mathrm{CD}$ spectra were minimized. From this fitting procedure, the relative positions and orientations of each dye are extracted (see Table S3.2).

Fitting the experimental absorbance (Figure 3.3a) and CD (Figure 3.3b) spectra for the adjacent dimer revealed that the dyes are oriented with predominantly J-aggregate (or head-to-tail) stacking but in a somewhat oblique manner, which is manifested in the increased vibronic peak at $600 \mathrm{~nm}$. This configuration supports the evidence provided by the red-shifted absorbance spectrum and demonstration of excitonic coupling in the $\mathrm{CD}$ spectrum, which only occur with an oblique stacking arrangement. Figure $3.3 \mathrm{c}$ visualizes the dimer stacking configuration within the $4 \mathrm{AJ}$ construct based on the fitting of the theory to the absorbance and CD data (see Table 3.3). The figure was created using UCSF Chimera visualization software. ${ }^{73,74}$ We note that only the position and orientation 
of the long axis of the Cy5 molecule is determined by the fitting procedure. Thus, how the molecules are oriented about the axis (i.e., rotated about the axis) and relative to the DNA is conjectural in Figures 3.3c, 3.3f, 3.3i, and 3.31. In contrast to the adjacent dimer, theoretical fitting of the transverse dimer absorbance (Figure 3.3d) and CD (Figure 3.3e) spectra using the KRM model determined that the dyes are indeed stacked parallel to one another (i.e., H-type) with a center-to-center distance of $\sim 1.0 \mathrm{~nm}$. The slight obliqueness of the dyes is exhibited in the EC-CD spectrum and a vibronic peak at $636 \mathrm{~nm}$ in the absorbance spectrum. Figure 3.3f illustrates the transverse dimer stacking configuration 
obtained from the theoretical fit of the absorbance and CD data.
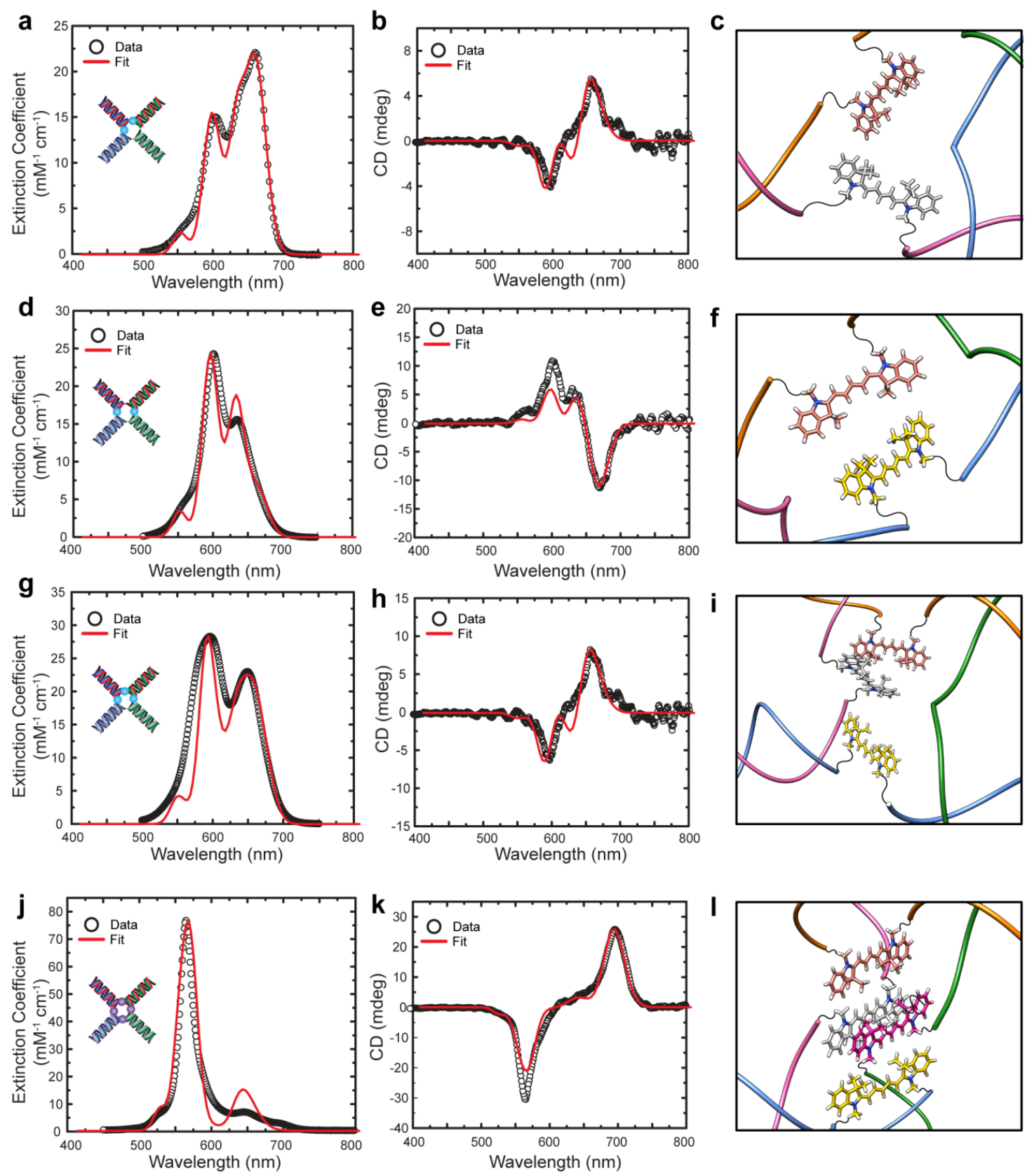

Figure 3.3. Absorbance (a,d,g, and j) and CD (b, e, h, and k) spectral fits with corresponding structures (c, $f$, i, and $l$ ) of the adjacent dimer, transverse dimer, trimer, and tetramer aggregates, respectively. Experimental data sets are shown as black open circles, while KRM theoretical fits are given as red curves. All samples were prepared at $10 \mu \mathrm{M}$ DNA concentration in a $1 \times$ TAE buffer solution with $15 \mathrm{mM}$ $\mathrm{MgCl} 2$ added, PAGE purified, and normalized by the resulting DNA concentrations $(\sim 0.5-2.5 \mu M)$. Fluorescence measurements were obtained by exciting samples at their respective absorption maxima. 
Using the KRM model to fit the trimer absorbance (Figure 3.3g) and CD (Figure 3.3h) spectra substantiates our hypothesis that there are not three underlying absorbance peaks, but rather the peak at $593 \mathrm{~nm}$ is simply broadened. Though the theoretical fit does not provide an explanation for the peak broadening, we can conjecture the existence of a population of trimer constructs having slightly different dye configurations (i.e., dye positions and orientations within the core of the $4 \mathrm{AJ}$ ), such that the spectrum, consisting of an average of these populations, exhibits a broadened absorption peak. The fit of the theory to the experimental absorbance and $\mathrm{CD}$ data indicates that the dyes of the trimer are stacked roughly parallel to each but with some twist, as depicted in Figure 3.3i. The configuration of the trimer dyes is predominantly H-type similar to the transverse dimer, which explains why their respective absorbance spectra appear so similar. Finally, fitting of the tetramer aggregate's absorbance (Figure 3.3j) and CD (Figure 3.3k) spectra incorporates the red-shifted peak at $690 \mathrm{~nm}$. Additionally, due to the strong EC-CD signals, obliqueness in the dye stacking arrangement was confirmed. The dye configuration obtained by the fitting procedure is illustrated in Figure 3.31. Input parameters, output positions and orientations for each dye, and a quantitative characterization of the quality of the theoretical fits are provided in the section 3.7.3.

\section{$\underline{\text { 3.3.3 Optical Reporter Functionality }}$}

To examine the viability of the $4 \mathrm{AJ}$ tetramer as a highly sensitive optical reporter, we constructed and studied the reaction kinetics of two reporter systems that exploit changes in fluorescence and optical absorption, respectively. In the system that exploited fluorescence changes, strand invasion was employed to disassemble a tetramer into a one dye and three dye complex. Strand invasion was facilitated by a designed $10 \mathrm{nt}$ extension 
(toehold) on one of the 4 arms to which the Invasion Strand can first attach by WatsonCrick base pairing. The Invasion Strand is fully complementary to the 4AJ strand with the extension and thus excises this strand from the $4 \mathrm{AJ} .{ }^{75}$ As illustrated in Figure 3.4a, this $4 \mathrm{AJ}$ tetramer (Tetramer - 1), would have nearly zero fluorescence while assembled. Upon invasion, the coherent excitonic delocalization within the aggregate would cease and greatly increased fluorescence would ensue from the dyes on both the single dye (monomer) product and the three dye complex (trimer). Conversely, the system that exploited absorbance changes consisted of two halves of the 4AJ synthesized separately that can hybridize when brought together to form a full 4AJ tetramer (Tetramer - 2). While disassembled, the two halves of the 4AJ (Dimer A and Dimer B) would exhibit minimal absorption at $565 \mathrm{~nm}$ (the H-tetramer absorbance peak), yielding a cyan solution color. When assembled, the absorption of the complete 4AJ (Tetramer - 2) at $565 \mathrm{~nm}$ greatly increases, yielding a solution color change to violet. This color change is depicted schematically in Figure 3.4 by the color of the represented dye molecules (cyan and violet spheres). 
a

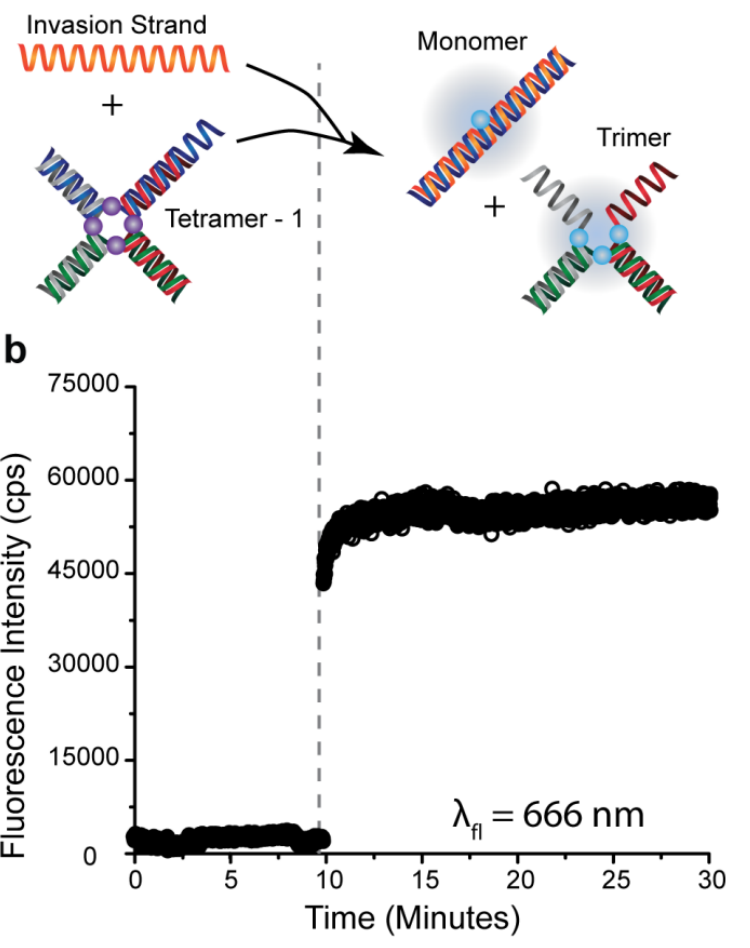

C

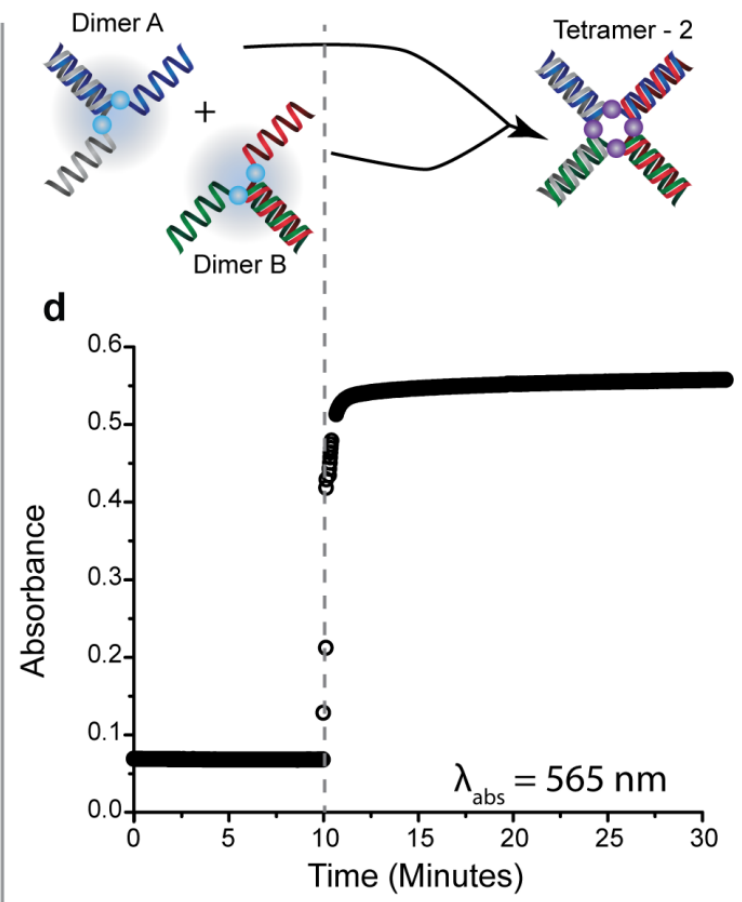

Figure 3.4. (a) Schematic illustration and (b) experimental demonstration of the 4AJ tetramer undergoing strand-invasion using fluorescence detection $(\lambda \mathrm{fl}=666 \mathrm{~nm})$. (c) Schematic illustration and (d) experimental demonstration of the $4 \mathrm{AJ}$ tetramer formation via DNA hybridization using UV-Vis absorbance, or colorimetric, detection $(\lambda a b s=565 \mathrm{~nm})$.

The operation of the fluorescence scheme (Figure 3.4b) yielded a 25-fold increase in fluorescence intensity upon disassembly of the $4 \mathrm{AJ}$ into a monomer and trimer.

Similarly, the absorbance reaction (Figure 3.4d) gave a 10-fold increase in absorbance at $565 \mathrm{~nm}$. For the DNA concentrations at which the experiments were carried out, $\sim 0.4 \mu \mathrm{M}$ for the fluorescence scheme and $\sim 0.1 \mu \mathrm{M}$ for the absorbance scheme, the time to half completion of the reactions was short compared to the 30 seconds required to thoroughly pipette mix the reactants in the cuvette. This implies a lower bound of $8 \times 10^{5} \mathrm{M}^{-1} \mathrm{~s}^{-1}$ for the rate constant of the strand invasion reaction of Figure 3.1a and a lower bound of 3 $\times 10^{5} \mathrm{M}^{-1} \mathrm{~s}^{-1}$ for the rate constant of the hybridization reaction of Figure 3.1c. These are 
consistent with the rate constants reported in the literature for these types of reactions, ${ }^{75}$, ${ }^{76}$ which are typically in the $10^{6} \mathrm{M}^{-1} \mathrm{~s}^{-1}$ range. The observed near complete $(>97.6 \%)$ fluorescence suppression and large Davydov splitting $(125 \mathrm{~nm}, 397.5 \mathrm{meV})$ in the $4 \mathrm{AJ}$ tetramer (Figure 3.2) signals its potential usefulness as a highly sensitive optical reporter.

\subsection{Experimental Methods}

\subsubsection{Synthesis}

Short 26 nucleotide (nt) DNA oligomers were purchased lyophilized from Integrated DNA Technologies, Inc. Oligomers internally functionalized with Cy5 dyes were purified via high-performance liquid chromatography (HPLC), while non dyefunctionalized strands were purified through standard desalting. All oligomers were rehydrated with ultrapure water (Barnstead Nanopure, Thermo Scientific) to produce a $100 \mu \mathrm{M}$ stock solution. DNA four-armed junctions (4AJs) were prepared by combining equimolar amounts of complementary oligomers in a $1 \times$ TAE $(40 \mathrm{mM}$ tris(hydroxymethyl)aminomethane, $20 \mathrm{mM}$ acetic acid, $1 \mathrm{mM}$ ethylenediaminetatraacetic acid) buffer solution ( $\mathrm{pH} 8.0)$ with $15 \mathrm{mM}$ added magnesium chloride $\left(\mathrm{MgCl}_{2}\right)$ to obtain a final DNA concentration of $10 \mu \mathrm{M}$. Resulting solutions were left for $24-48$ hours at room temperature to allow for hybridization and self-assembly. TAE (10× stock solutions) and $\mathrm{MgCl}_{2}$ (99\% purity) were purchased from Fisher Scientific and used as received. Solutions of $1.375 \mathrm{M} \mathrm{MgCl}_{2}$ were added to a $1 \times \mathrm{TAE}$ buffer solution to yield final magnesium $\left(\mathrm{Mg}^{2+}\right)$ concentrations of 0,15 , and $100 \mathrm{mM}$. The initial salt concentration due to the TAE buffer ( $<1 \mathrm{mM} \mathrm{Na}^{+}$arising from the EDTA counterion) was found to be negligible compared to the subsequently added salt concentrations. 


\section{$\underline{\text { 3.4.2 Purification }}$}

Self-assembled DNA 4AJ structures were further purified using polyacrylamide gel-electrophoresis (PAGE). Solutions of 4AJs were prepared with various dye arrangements in a $1 \times$ TAE buffer at $10 \mu \mathrm{M}$ double-stranded DNA (dsDNA) concentrations and $15 \mathrm{mM}$ added $\mathrm{MgCl}_{2}$. A 6× New England Biolabs loading buffer was added in a 1:5 ratio with the $4 \mathrm{AJs}$ to give a resulting $1 \times$ concentration of the loading buffer ( $1 \times$ buffer: $11 \mathrm{mM}$ ethylenediaminetetraacetic acid, $3.3 \mathrm{mM}$ tris-hydrochloric acid, $0.017 \%$ sodium dodecyl sulfate, $0.015 \%$ bromophenol blue, $2.5 \%$ Ficoll®- 400$)$. The prepared sample solutions were then injected into a $12 \%$ gel, prepared with $0.25 \times \mathrm{TAE}$ with $3.75 \mathrm{mM} \mathrm{MgCl} 2$ added and allowed to migrate for approximately 90 minutes with a $150 \mathrm{~V}$ applied voltage. $1 \times \mathrm{TAE}$ with $12 \mathrm{mM}$ added $\mathrm{MCl}_{2}$ was used in the buffer reservoirs of the electrophoresis apparatus. Using a multiplexed gel imaging and documentation system (FluorChem Q, ProteinSimple), completed gels were imaged by brief illumination with a $254 \mathrm{~nm}$ ultraviolet (UV) light source while placed on a phosphor imaging plate. Well-formed 4AJ structures were identified, excised from the gels with a razor blade, and extracted. Extraction was conducted by placing select excised gel pieces into a vial of $1 \times \mathrm{TAE}$ buffer containing $15 \mathrm{mM}$ added $\mathrm{MgCl}_{2}$. The DNA $4 \mathrm{AJ}$ s were allowed 48 hours to diffuse out of the gel and into the surrounding buffer solution. The resulting buffer solution was then extracted from the vial and any remaining gel fragments removed by centrifuging the sample at 1000 relative centrifugal force (rcf) for 10 minutes. A further description of the gel-electrophoresis process and gel images are provided in section 3.7.5. 


\subsubsection{Optical Characterization}

All optical characterization was performed at room temperature. UV-Vis spectra were obtained using a dual-beam Cary 5000 UV-Vis-NIR spectrophotometer (Agilent Technologies). Solution samples $(150 \mu \mathrm{L})$ were transferred into a $1 \mathrm{~cm}$ path length submicro, self-masking quartz spectrophotometer cell (Starna). Static absorbance spectra were monitored over a range of wavelengths and normalized by DNA concentrations. Static fluorescence spectra were obtained using a Fluorolog-3 spectrofluorometer (Horiba Scientific) and monitored as a function of wavelength. PAGE purified solution samples of each $4 \mathrm{AJ}$ construct $(150 \mu \mathrm{L})$ were transferred to a $1 \mathrm{~cm}$ path length sub-micro, selfmasking fluorometer cell (Starna) and were excited at or near their respective absorption maxima listed in Figure 2. The excitation and emission bandwidths used were $5.0 \mathrm{~nm}$ and $2.5 \mathrm{~nm}$, respectively. Circular dichroism (CD) measurements were performed using a JACSO-J810 spectropolarimeter. Samples of purified 4AJ constructs $(100 \mu \mathrm{L})$ were transferred to a $1 \mathrm{~cm}$ path length micro, self-masking quartz cuvette (Thor Labs). Spectra were monitored over a series of wavelengths and normalized by the DNA concentration.

\subsubsection{Optical Reporter Studies}

Optical reporter studies were performed using two characterization techniques: fluorescence and absorbance. For fluorescence studies, DNA-templated 4AJs were designed with a $10 \mathrm{nt}$ toehold on one of the four arms. Structures were synthesized and purified as described above. Spectra were collected using a Horiba Fluorolog-3 spectrofluorometer. Static fluorescence spectra of the samples were obtained prior to and subsequently following the reaction. Samples $(250 \mu \mathrm{L})$ were monitored at the fluorescence peak maximum $(666 \mathrm{~nm})$ over 70 minutes while being excited at the 
tetramer absorbance peak maximum $(565 \mathrm{~nm})$. At 10 minutes, an invasion strand designed to be complementary to the strand with the $10 \mathrm{nt}$ toehold extension - was introduced into the system in 1:1 stoichiometry with the $4 \mathrm{AJ}$ and pipette mixed for $\sim 30$ seconds. The change in fluorescence intensity was then monitored over 70 minutes. The initial dsDNA concentrations of the $4 \mathrm{AJ}$ were $\sim 0.4 \mu \mathrm{M}$, and all reactions were held at 22 ${ }^{\circ} \mathrm{C}$.

Absorbance studies were conducted by initially synthesizing the $4 \mathrm{AJ}$ structure in two parts, described as Dimers A and B, such that the two dimers are complementary and could join together to form the full 4AJ structure. Dimer A and B were purified via PAGE and diluted to $\sim 0.1 \mu \mathrm{M}$ dsDNA concentration. Using a Cary 5000 UV-Vis-NIR spectrophotometer, changes in absorbance at the H-tetramer peak maximum (565 nm) were monitored. Initially, a sample of Dimer A was monitored. At 10 minutes, Dimer B was introduced in stoichiometry and pipette mixed for $\sim 30$ seconds. Upon hybridization the $4 \mathrm{AJ}$ tetramer formed, inducing an absorbance signal at $565 \mathrm{~nm}$. All reactions were held at room temperature $\left(22^{\circ} \mathrm{C}\right)$.

\subsubsection{Stability Studies}

Results from studies performed to monitor the stability of the 4AJs with changes in solution salt and DNA concentrations are described in section 3.7.6. Samples of tetramer $4 \mathrm{AJ}$ constructs used for salt studies were prepared in a $1 \times \mathrm{TAE}$ buffer solution containing 0-100 $\mathrm{mM} \mathrm{MgCl} 2$ and held at a constant DNA concentration of $1.0 \mu \mathrm{M}$. Samples of the tetramer used for DNA concentration studies were prepared in a $1 \times$ TAE buffer solution with $0.1-3.0 \mu \mathrm{M}$ dsDNA concentrations and held at a constant salt concentration of $15 \mathrm{mM} \mathrm{MgCl}$. 


\subsection{Conclusion}

We have explored a two-dimensional DNA template for proximally positioning molecular dyes to induce exciton delocalization. The four-armed junction (4AJ) DNA construct enabled the analysis of a variety of dye aggregate types (i.e., adjacent and transverse dimers, trimer, and tetramer) that revealed various exciton delocalization spectral signatures. Through static spectral characterization of the 4AJ-templated dye aggregates, it was found that the type of aggregate (i.e., H-aggregate, J-aggregate, or oblique) depended largely on the position of the dyes within the $4 \mathrm{AJ}$ construct. The specificity of the dye position was demonstrated explicitly by the difference in spectral properties of the two dimer constructs, such that the dimer with dyes positioned on adjacent arms (adjacent dimer) demonstrated predominantly J-aggregate behavior, whereas the transverse dimer with dyes positioned on opposite arms exhibited Haggregate behavior. The tetramer, with four dyes positioned at each node of the $4 \mathrm{AJ}$, expressed a strikingly large Davydov splitting and strong fluorescence suppression. Exploiting the distinctive optical properties of the tetramer afforded the opportunity to fabricate and operate two dynamic dye aggregate schemes for potential use as a sensitive optical reporter using either absorbance or fluorescence characterization methods. Further exploration of exciton delocalization in DNA-templated dye assemblies may yield applications in optical detection and imaging schemes, light-harvesting, photovoltaics, optical information processing, and quantum computing.

\subsection{Acknowledgments}

The authors would like to thank the students and staff within the Nanoscale Materials \& Device group for valuable assistance with this work, specifically Dr. Natalya 
Hallstrom and Allison Christy for their help with PAGE Gel electrophoresis experiments, J. Melinger and colleagues at the U.S. Naval Research Laboratory (NRL) for helpful discussions regarding the theoretical work of O. Kühn et al. and L. Li, E. Nelson, and A. Correa for DFT modeling. The research was supported in part by the Boise State University Division of Research and Economic Development, the National Science Foundation INSPIRE No. 1648655, and the NASA Idaho Space Grant Consortium (ISGC). We acknowledge the Biomolecular Research Center for the use of their CD instrumentation supported in part by the Institutional Development Awards (IDeA) from the National Institute of General Medical Sciences No. P20GM103408 and the National Institutes of Health No P20GM109095, the National Science Foundation No. 0619793 and 0923535, the MJ Murdock Charitable Trust, the Idaho State Board of Education. B.L.C. acknowledges the Micron Technology MSE Ph.D. Fellowship. The molecular graphics and analyses were performed using the UCSF Chimera package, which is developed by the Resource for Biocomputing, Visualization, and Informatics at the University of California, San Francisco.

\subsection{Supporting Information}

\subsubsection{Strand Sequences and Dye Details}

The sequences for both the dye labeled (i.e., Cy5) and non-dye labeled DNA oligomers are given in Table 3.2 below. Oligonucleotides were purchased from Integrated DNA Technologies, Inc. (IDT, Coralville, IA, USA). The Cy5 structure is illustrated in Figure 3.5, and shows the attachment sites by which the dye is covalently bound to the 5' and 3' sides of the DNA backbone, respectively. The final structure of the assembled Holliday junction (i.e., 4AJ) is illustrated in Figure 3.6, along with schematics 
of the constructs used in the absorbance and fluorescence detection schemes. All strands with an internally functionalized dye were purified using high-performance liquid chromatography (HPLC), while all non-dye labeled strands were purified using a standard desalting procedure.

Table 3.2 DNA Oligomer Sequences

\begin{tabular}{|c|c|c|c|}
\hline $\begin{array}{c}\text { Strand } \\
\text { Name }\end{array}$ & Sequence (5' to 3') & $\begin{array}{c}\text { Length } \\
\text { (nt) }\end{array}$ & Purification \\
\hline A & ATATAATCGCTCGCATATTATGACTG & 26 & Standard \\
\hline B & CAGTCATAATATGTGGAATGTGAGTG & 26 & Standard Desalting \\
\hline C & CACTCACATTCCACTCAACACCACAA & 26 & Standard Desalting \\
\hline D & TTGTGGTGTTGAGCGAGCGATTATAT & 26 & Standard Desalting \\
\hline Cy5-A & ATATAATCGCTCG/iCy5/CATATTATGACTG & 26 & HPLC** \\
\hline Cy5-B & CAGTCATAATATG/iCy5/TGGAATGTAGTG & 26 & HPLC \\
\hline Cy5-C & CACTCACATTCCA/iCy5/CTCAACACCACAA & 26 & HPLC \\
\hline Cy5-D & TTGTGGTTTGAG/iCy5/CGAGCGATTATAT & 26 & HPLC \\
\hline Cy5-B2 & CAGTCATAATATGTGGAATGTAGTGTAAAGCCGTT & 36 & HPLC \\
\hline
\end{tabular}

\footnotetext{
* Desalting to remove short products and small organic contaminants. Does include PAGE purification

** High-Performance Liquid Chromatography
}

The common name and structure of the dye used in this study is:

Cy5: 1-[3-(4-monomethoxytrityloxy)propyl]-1'-[3-[(2-cyanoethyl)-(N,Ndiisopropyl phosphoramidityl]propyl]-3,3,3',3'-tetramethylindodicarbocyanine chloride. 


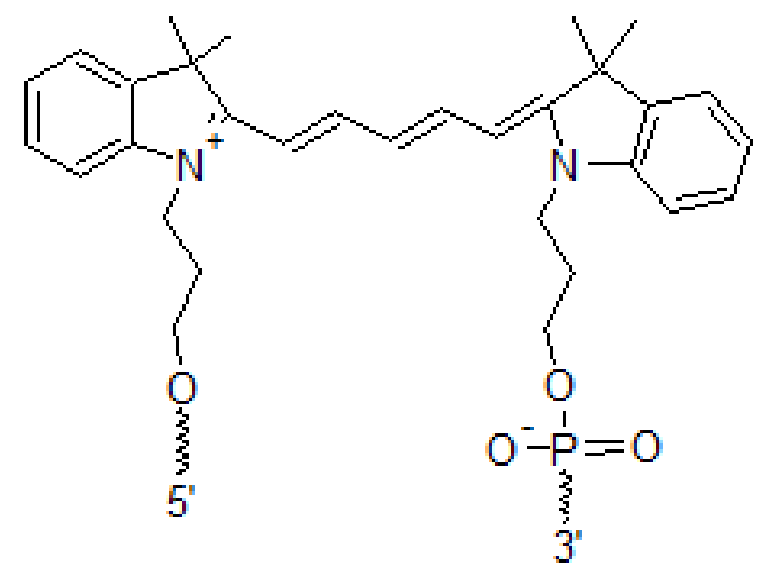

Figure 3.5. Molecular structure of the Cy5 dye adapted from IDT. The two long carbon tethers represent the directionality of the attachment scheme onto the phosphate backbone of the DNA.

a

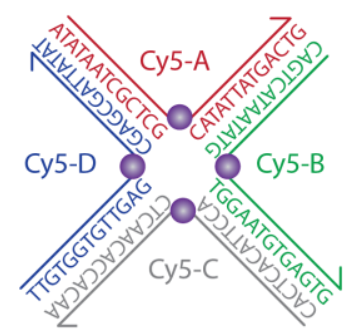

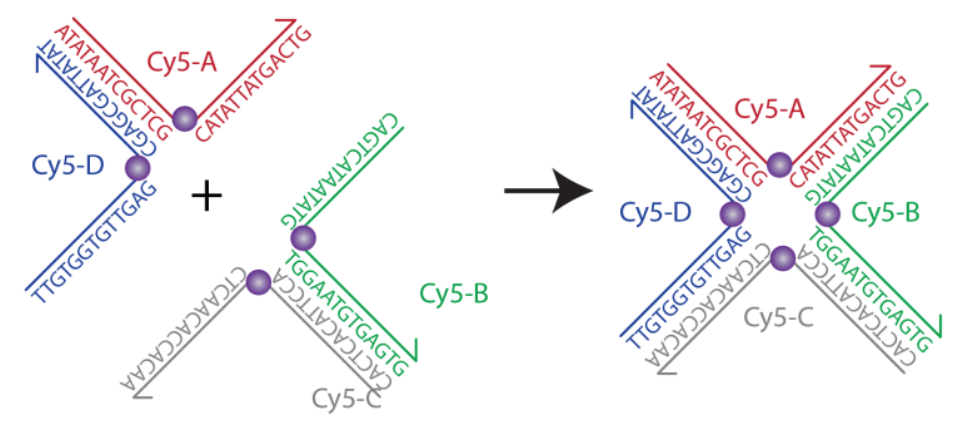

c

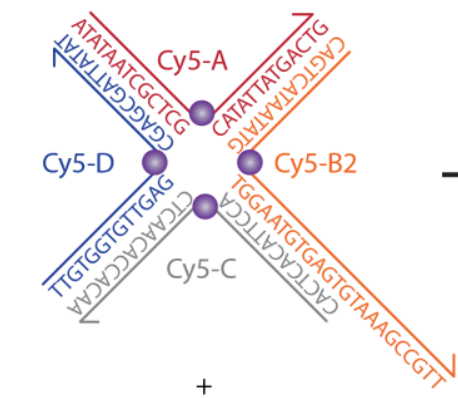

$\frac{\text { B2-comp }}{\text { AACGGCTTTACACTCACATTCCACATATTATGACTG }}$

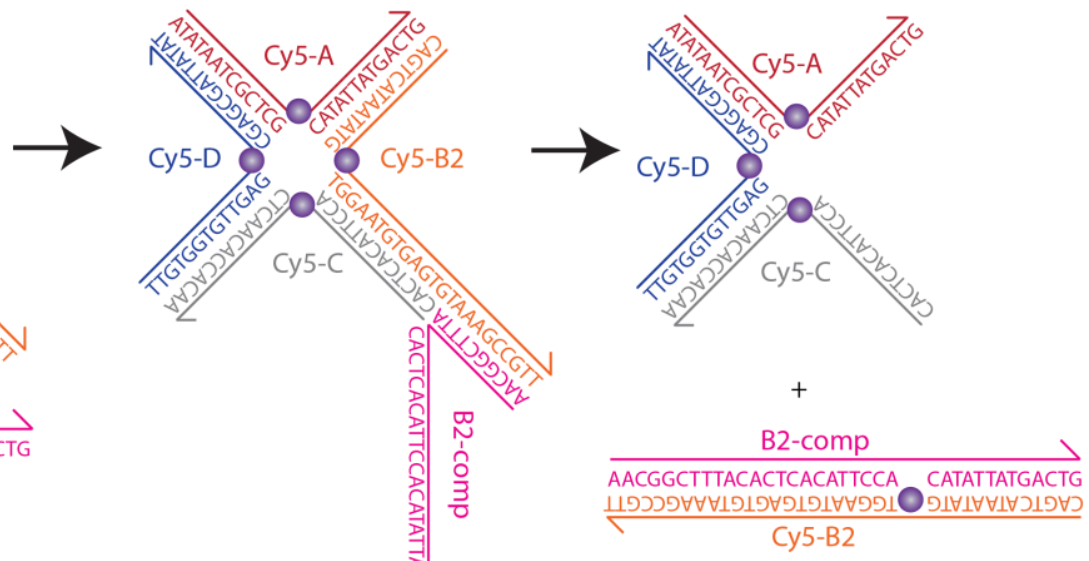

it

Figure 3.6. Illustration depicting the complementary DNA domains of each strand (Cy5-A, Cy5-B, Cy5-C, Cy5-D, etc.) that hybridize to form the (a) complete fourarmed junction (4AJ) structure, (b) absorbance detection scheme, and (c) fluorescence detection scheme. The locations of $\mathrm{Cy} 5$ molecules are indicated by purple spheres; however, do not suggest orientation. 


\subsubsection{Spectral Analysis of Absorbance and Fluorescence Data}

UV-Vis absorbance and fluorescence spectra for each 4AJ construct (Figure 3.7) were analyzed by fitting each dataset to a series of Gaussian curves using a least squares fitting procedure, where a Gaussian curve is defined by:

$$
y=y_{0}+\left(\frac{A}{w \sqrt{\frac{\pi}{2}}}\right) e^{-2\left(\frac{x-x_{c}}{w}\right)^{2}}
$$

Here, $\mathrm{y}_{0}$ is an offset given in $\mathrm{mM}^{-1}$ or counts. $\mathrm{mM}^{-1}$ for absorbance and fluorescence spectra, respectively. The area of the curve is $A$, the position of the peak is denoted by $x_{c}$, and the width of the peak is $w$. The FWHMs are calculated as $w \sqrt{\ln (4)}$ and listed in Table 3.3. Gaussian fit parameters and residuals are provided in Table 3.3 for each 4AJ construct's absorbance (Abs) and fluorescence (FL) peak maximum.

Samples used to obtain absorbance and fluorescence spectra were PAGE purified and normalized by dsDNA concentration prior to performing a fitting procedure on each absorbance and fluorescence dataset.

Fluorescence suppression is calculated using the integrated area of the emission curves given by:

$$
\% F L \text { Suppression }=\frac{\int F L_{\text {monomer }}-\frac{1}{n} \int F L_{\text {aggregate }}}{\int F L_{\text {monomer }}} \cdot 100
$$

where $\int F L_{\text {monomer }}$ is the integrated area of the monomer emission curve and $\int F L_{\text {aggregate }}$ is the integrated area under the aggregate (i.e., dimer, trimer, or tetramer) emission curve. The aggregate fluorescence is normalized by dye concentration, $n$. Integration was performed between a range of $650 \mathrm{~nm}$ and $800 \mathrm{~nm}$ wavelengths. 

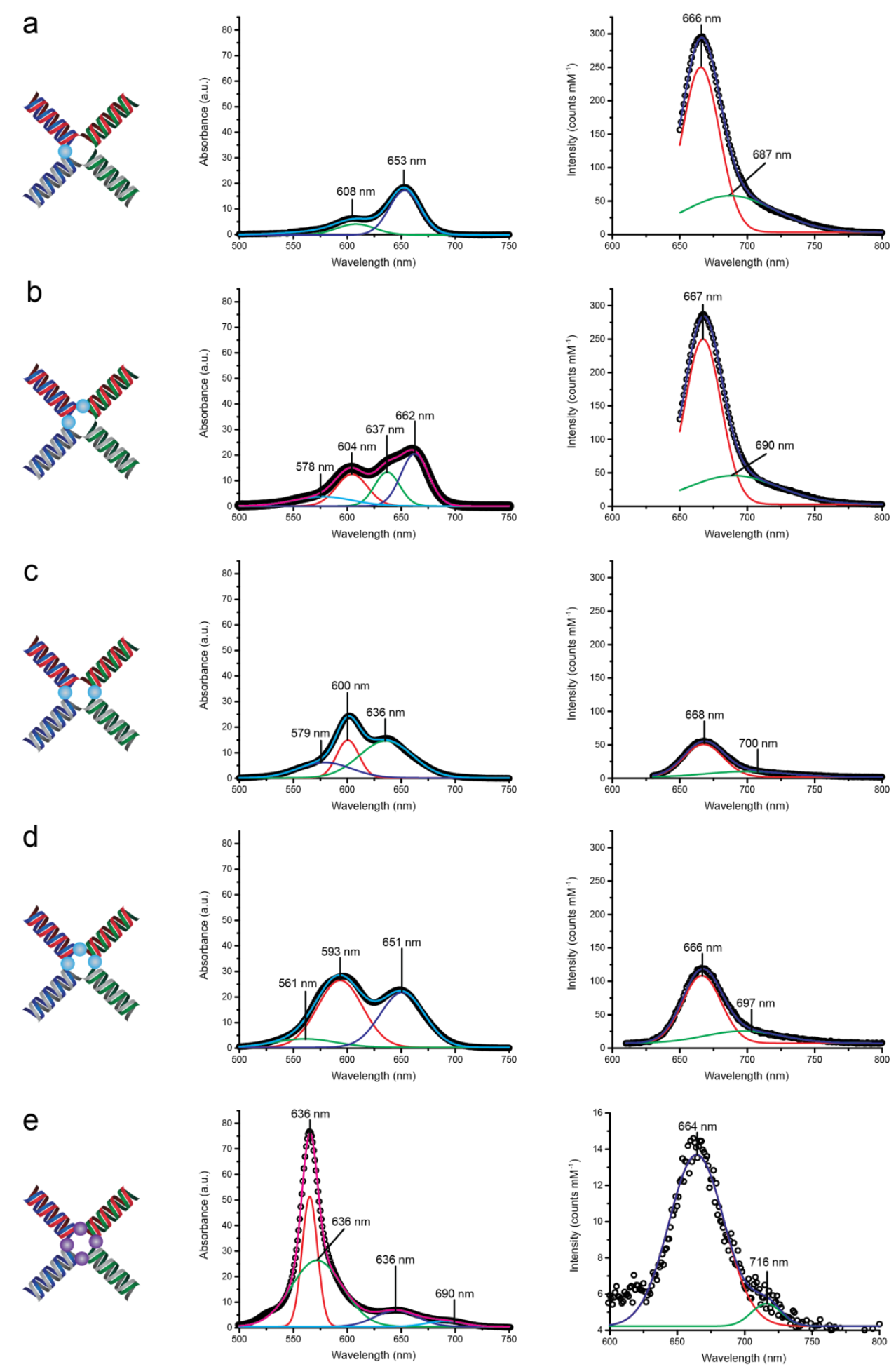

Figure 3.7. Peak fitting performed on both absorbance (left panels) and fluorescence (right panels) spectra (black circles) using a Gaussian curve (solid curves) and a least squares fitting procedure for the $4 \mathrm{AJ}$ (a) monomer, (b) adjacent dimer, (c) transverse dimer, (d) trimer, and (e) tetramer configurations. The fluorescence studies were performed by monitoring the emission over a range of wavelengths and exciting the aggregates at their respective absorbance maxima. 
Table 3.3 Gaussian fitting parameters obtained for the peak maximum of each absorbance (Abs) and fluorescence (FL) data set shown in Figure 3.7.

\begin{tabular}{|c|c|c|c|c|c|c|}
\hline \multirow[t]{2}{*}{$\begin{array}{c}\text { 4AJ } \\
\text { Construct }\end{array}$} & \multicolumn{6}{|c|}{$\begin{array}{c}\text { Gaussian Fit Equation } \\
y=y_{0}+\left(\frac{A}{\sqrt{\pi}}\right) e^{-2\left(\frac{x-x_{c}}{w}\right)^{2}}\end{array}$} \\
\hline & $\mathrm{y}_{0}$ & $x_{c}(\mathrm{~nm})$ & $w(\mathrm{~nm})$ & $A$ & $\begin{array}{c}\text { Adjusted } \\
\mathrm{R}^{2}\end{array}$ & $\begin{array}{c}\text { FWHM } \\
(\mathrm{nm})\end{array}$ \\
\hline $\begin{array}{l}\text { Monomer } \\
(\mathrm{Abs})\end{array}$ & $\begin{array}{c}(-1.6 \pm 0.3) \\
\times 10^{3}\end{array}$ & $653.1 \pm 0.1$ & $28.7 \pm 0.1$ & $\begin{array}{c}(6.3 \pm 0.6) \\
\times 10^{6}\end{array}$ & 0.999 & $\begin{array}{c}33.8 \pm \\
0.2\end{array}$ \\
\hline $\begin{array}{l}\text { Monomer } \\
(\mathrm{FL})\end{array}$ & $3.5 \pm 0.5$ & $665.6 \pm 0.2$ & $27.7 \pm 0.5$ & $\begin{array}{c}(8.6 \pm 0.1) \\
\times 10^{3} \\
\end{array}$ & 0.999 & $\begin{array}{c}32.6 \pm \\
0.6\end{array}$ \\
\hline $\begin{array}{l}\text { Adjacent } \\
\text { Dimer } \\
(\text { Abs })\end{array}$ & $\begin{array}{c}(1.2 \pm 0.2) \\
\times 10^{3}\end{array}$ & $662.5 \pm 0.2$ & $25.7 \pm 0.2$ & $\begin{array}{c}(6.6 \pm 0.2) \\
\times 10^{6}\end{array}$ & 0.999 & $\begin{array}{c}30.3 \pm \\
0.2\end{array}$ \\
\hline $\begin{array}{l}\text { Adjacent } \\
\text { Dimer } \\
(\mathrm{FL})\end{array}$ & $3.0 \pm 0.3$ & $667.3 \pm 0.1$ & $27.0 \pm 0.3$ & $\begin{array}{c}(8.4 \pm 0.3) \\
\times 10^{3}\end{array}$ & 0.999 & $\begin{array}{c}31.8 \pm \\
0.4\end{array}$ \\
\hline $\begin{array}{l}\text { Transverse } \\
\text { Dimer } \\
\text { (Abs) }\end{array}$ & $\begin{array}{c}(1.6 \pm 0.3) \\
\times 10^{3}\end{array}$ & $600.5 \pm 0.1$ & $20.7 \pm 0.3$ & $\begin{array}{c}(3.8 \pm 0.2) \\
\times 10^{6}\end{array}$ & 0.999 & $\begin{array}{c}24.3 \pm \\
0.4\end{array}$ \\
\hline $\begin{array}{l}\text { Transverse } \\
\text { Dimer } \\
\text { (FL) }\end{array}$ & $1.84 \pm 0.1$ & $667.6 \pm 0.1$ & $29.1 \pm 0.3$ & $\begin{array}{c}(1.8 \pm 0.1) \\
\times 10^{3}\end{array}$ & 0.999 & $\begin{array}{c}34.3 \pm \\
0.3\end{array}$ \\
\hline $\begin{array}{c}\text { Trimer } \\
(\mathrm{Abs})\end{array}$ & $\begin{array}{c}(3.0 \pm 0.6) \\
\times 10^{3} \\
\end{array}$ & $593.3 \pm 1.0$ & $41.8 \pm 1.6$ & $\begin{array}{c}(13.7 \pm \\
2.7) \times 10^{6}\end{array}$ & 0.998 & $\begin{array}{c}49.2 \pm \\
1.8 \\
\end{array}$ \\
\hline $\begin{array}{l}\text { Trimer } \\
(\mathrm{FL})\end{array}$ & $7.4 \pm 0.2$ & $666.3 \pm 0.1$ & $28.7 \pm 0.3$ & $\begin{array}{c}(3.6 \pm 0.1) \\
\times 10^{3}\end{array}$ & 0.998 & $\begin{array}{c}33.8 \pm \\
0.4\end{array}$ \\
\hline $\begin{array}{c}\text { Tetramer } \\
(\mathrm{Abs})\end{array}$ & $\begin{array}{c}(1.9 \pm 0.3) \\
\times 10^{3}\end{array}$ & $564.7 \pm 0.1$ & $13.5 \pm 0.1$ & $\begin{array}{c}(7.9 \pm 0.1) \\
\times 10^{6}\end{array}$ & 0.999 & $\begin{array}{c}15.9 \pm \\
0.1\end{array}$ \\
\hline $\begin{array}{l}\text { Tetramer } \\
\text { (FL) }\end{array}$ & $4.2 \pm 0.1$ & $664.4 \pm 0.4$ & $41.0 \pm 1.0$ & $\begin{array}{c}(4.9 \pm 0.1) \\
\times 10^{2}\end{array}$ & 0.952 & $\begin{array}{c}48.3 \pm \\
1.2\end{array}$ \\
\hline
\end{tabular}




\subsubsection{Fitting the Absorption and Circular Dichroism (CD) data using the Kühn-Renger-}

\section{May Model}

Following the same approach as that used in our previous work, ${ }^{68}$ which is guided by the theoretical work of Kühn, Renger, and May ${ }^{69}$ we model the absorption and circular dichroism (CD) spectra that singles out a dominant vibrational mode in each dye and treats the mode separately from the vibrational continuum by including it as part of the system Hamiltonian. This approach corresponds to standard expressions commonly used in exciton-vibrational problems; however, the vibrational eigenstate avoids perturbation treatment of the exciton-vibrational coupling. Furthermore, a dipose approximation is inadequate since the distance between the dyes can be less than the dye length; therefore, a separated charge approximation was used in the model. ${ }^{68}$

From the derivation provided in our previous work, we find the absorbance as a function of energy $E$, for comparison with experimental data, which is computed by convolving the discrete spectrum of energy eigenvalues, $E_{i}$, obtained by diagonalzing the model Hamiltonian, $H$, with a Gaussian:

$$
A(E)=\sum_{i} \frac{\gamma_{i}}{\sqrt{2 \pi \Gamma^{2}}} \exp \left(-\frac{\left(E-E_{i}\right)^{2}}{2 \Gamma^{2}}\right),
$$

where $\gamma_{i}$ are the transition rates between the ground state and $E_{i}$. Similarly, the differential $\mathrm{CD}$ absorbance as a function of energy was computed as:

$$
A_{C D}(E)=\sum_{i} \frac{\gamma_{i}^{C D}}{\sqrt{2 \pi \Gamma^{2}}} \exp \left(-\frac{\left(E-E_{i}\right)^{2}}{2 \Gamma^{2}}\right),
$$

where the value of the damping constant due to energy loss $(\Gamma)$ was adjusted to give the best fit to all the data. This provides a phenomenological approximation on the effect that the vibronic continuum has on the spectra. 
The phenomenological constants entering the theoretical model were the same ones used in reference [68]. In particular, by fitting the monomer spectra, it had been determined parameters specific to the Cy5 dye, such as the wavelength of the peak of the monomer spectrum, the energy of the vibronic peak $E_{v i b}=0.16 \mathrm{eV}$, and the horizontal displacement $d=0.78$ of the excited electronic state vibrational potential from that of the ground electronic state vibrational potential. Fits to the dimer spectrum fixed the values for the exchange coupling constant $J=0.054 \mathrm{eV} \mathrm{nm}^{3}$ and for the line-width parameter $\Gamma=0.038 \mathrm{eV}$. We used these values of the model parameters for all the other aggregates. The cut-off for the vibrational state Hilbert spaces was set to $n_{v}=3$, that is, vibrational states with the vibration quantum numbers $0,1,2$ were included for both the ground and excited electronic state manifold for each dye. In addition, it was assumed that there were no changes in the electronic state energy shifts between the ground electronic states and the excited electronic states resulting from changes in the supramolecular environment of the dyes for the different dye configurations, that is, the parameter $E_{0}$ was set to zero.

A stochastic gradient-search algorithm was used to search for the dye configurations that resulted in the best fit between the theoretical and experimental absorbance and CD spectra. In order to avoid solutions in which the dyes are unphysically close, the search was restricted to configurations in which the distance of closest approach between the long axes of any pair of dyes is greater than $0.45 \mathrm{~nm}$. The fitness function consisted of a sum of three terms. The first term sought to enforce the ratio of the peak height of the $\mathrm{CD}$ spectrum to that of the absorbance spectrum to be the same for both the experimental and the theoretical spectra. The second term sought to minimize the mean-square error between the experimental and theoretical absorbance 
spectrum and third term sought to minimize the mean-square error between the experimental and theoretical CD spectrum. The term that minimized the mean-square error between the experimental and theoretical absorbance spectra was weighted more heavily than the other terms since this data had the least noise.

Table 3.3 lists parameters indicating the goodness of the fits that were obtained for the various dye-DNA constructs. Let $P_{C D}$ denote the height of the highest peak of the absolute value of the $\mathrm{CD}$ spectrum and let $P_{A}$ denote the height of the highest peak of the absorbance spectrum. Let $r^{\text {th }}$ denote the ratio for $P_{C D}$ to $P_{A}$ for the theoretical spectrum and $r_{e x}$ the ratio of PCD to PA for the experimental spectrum, then $\mathrm{r}$, listed in Table 3.3 is given by $r=r_{t h} / r_{e x}$. This quantity would be 1 if there were perfect agreement between the theoretical and experimental spectra. As a dimensionless way of reporting the goodness of fits to the absorbance and $\mathrm{CD}$ spectra the overlap integrals between corresponding spectra were evaluated. Letting $S_{a b, e x}(E), S_{a b, t h}(E), S_{c d, e x}(E)$, and $S_{c d, t h}(E)$ denote respectively the experimental absorbance spectrum, theoretical absorbance spectrum, experimental $\mathrm{CD}$ spectrum, and theoretical $\mathrm{CD}$ spectrum where $\mathrm{E}$ is energy, the normalized absorbance overlap integral $\left(\mathrm{OI}_{\mathrm{AB}}\right)$ of the spectrum is defined by:

$$
O I_{A B}=\frac{\int S_{a b, e x}(E) S_{a b, t h}(E) d E}{\sqrt{\int S_{a b, e x}^{2}(E) d E} \sqrt{\int S_{a b, t h}^{2}(E) d E}} .
$$

and the normalized overlap integral for the $\mathrm{CD}$ spectrum $\left(\mathrm{OI}_{\mathrm{CD}}\right)$ is defined by:

$$
O I_{C D}=\frac{\int S_{c d, e x}(E) S_{c d, t h}(E) d E}{\sqrt{\int S_{c d, e x}^{2}(E) d E} \sqrt{\int S_{c d, t h}^{2}(E) d E}} .
$$

To provide an overall goodness of fit parameter $\left(\mathrm{OI}_{\mathrm{Tot}}\right)$, we introduce:

$$
O I_{T o t}=\frac{1}{2}\left(O I_{a b}+O I_{c d}\right) .
$$


The normalized overlap integrals provide a measure of how closely the experimental and theoretical curves match and must be less than or equal to one. Only for a perfect match are they equal to one.

The angle and position of each dye is output from the resulting fit, provided in units of angstroms and degrees, respectively, and listed in Table 3.4. From the angles, which are given in spherical coordinates $(\theta, \varphi)$, the Cartesian components of the molecular orientation vector (i.e., the unit vector parallel to the long axis of the Cy5 molecule) can be computed using the following set of equations:

$$
\begin{aligned}
& n_{x}=\sin (\theta) \cos (\varphi), \\
& n_{y}=\sin (\theta) \sin (\varphi), \\
& n_{z}=\cos (\theta)
\end{aligned}
$$

Table 3.4 Goodness of fit parameters for absorbance and CD spectra of various 4AJ configurations.

\begin{tabular}{|c|c|c|c|c|}
\hline $\begin{array}{c}\text { Dye-DNA } \\
\text { Construct }\end{array}$ & \multicolumn{4}{|c|}{ Kühn-Renger-May Model Parameters } \\
\hline & $r$ & $O I_{A B}$ & $O I_{C D}$ & $O I_{T o t}$ \\
\hline Adjacent Dimer & 1.02 & 0.995 & 0.758 & 0.876 \\
\hline $\begin{array}{c}\text { Transverse } \\
\text { Dimer }\end{array}$ & 1.06 & 0.983 & 0.946 & 0.965 \\
\hline Trimer & 1.03 & 0.964 & 0.903 & 0.933 \\
\hline Tetramer & 1.03 & 0.978 & 0.981 & 0.980 \\
\hline
\end{tabular}


Table 3.5 Kühn-Renger-May model fitting outputs describing each dye orientation and position.

\begin{tabular}{|c|c|c|c|c|c|}
\hline $\begin{array}{c}\text { Dye-DNA } \\
\text { Construct }\end{array}$ & \multicolumn{5}{|c|}{ Kühn-Renger-May Model Outputs } \\
\hline & $\theta$ (degrees) & $\varphi$ (degrees) & $x(\AA)$ & $y(\AA)$ & $z(\AA)$ \\
\hline Adjacent Dimer & $-45.43^{\circ}$ & $-132.08^{\circ}$ & 0.00 & 0.00 & 0.00 \\
Dye 1 & $63.18^{\circ}$ & $-155.54^{\circ}$ & 14.14 & 0.00 & 0.00 \\
Dye 2 & & & & & \\
\hline Transverse Dimer & $-10.94^{\circ}$ & $5.42^{\circ}$ & 0.00 & 0.00 & 0.00 \\
\hline Dye 1 & $31.97^{\circ}$ & $3.68^{\circ}$ & 10.09 & 0.00 & 0.00 \\
Dye 2 & & & & & \\
Trimer & $24.91^{\circ}$ & $-8.65^{\circ}$ & 10.33 & 0.43 & -13.41 \\
Dye 1 & $48.73^{\circ}$ & $20.17^{\circ}$ & 16.27 & -2.93 & -16.66 \\
Dye 2 & $50.12^{\circ}$ & $-201.16^{\circ}$ & 28.88 & -5.81 & -13.12 \\
Dye 3 & & & & & \\
\hline Tetramer & $2.06^{\circ}$ & $1.78^{\circ}$ & 0.25 & -0.15 & 1.98 \\
Dye 1 & $2.83^{\circ}$ & $1.73^{\circ}$ & 8.70 & -2.81 & 2.85 \\
Dye 2 & $9.16^{\circ}$ & $4.04^{\circ}$ & 9.31 & 1.86 & 2.73 \\
Dye 3 & $8.28^{\circ}$ & $179.74^{\circ}$ & 17.50 & -0.52 & 4.92 \\
Dye 4 & \multicolumn{3}{|c|}{} \\
\hline
\end{tabular}

\subsubsection{Optical Reporter}

As shown in Figure 3.8, reaction kinetics were performed to demonstrate two types of transitions: (i) non-fluorescent and fluorescent states (Figure 3.8a) and (ii) nonabsorbent and absorbent at $562 \mathrm{~nm}$, thus transitioning from a cyan solution color to violet (Figure 3.8b). Lower bounds on the reaction rate constants $k$ were computed using:

$$
k>\frac{1}{t_{m[D N A]}},
$$

where $t_{\mathrm{m}}$ is the mixing time and [DNA] is the concentration of one of the DNA reactants, since the reactants are in stoichiometry and undergo a second order reaction. 
a
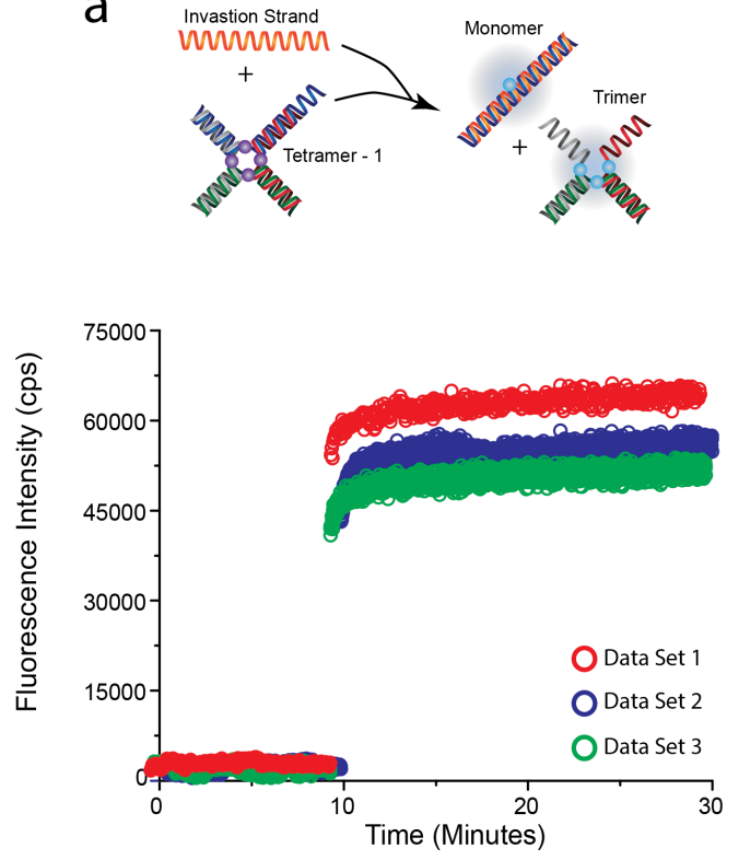

b
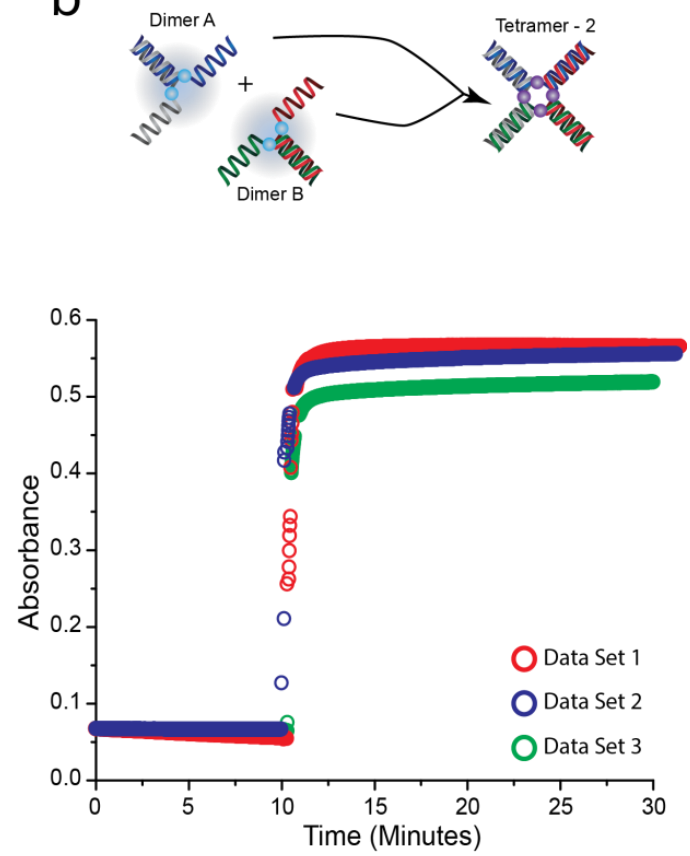

Figure 3.8. Optical reporter data demonstrating transitions between nonfluorescent and fluorescent states for the fluorescence-based detection scheme (a) and between non-absorbing and absorbing states for the absorbance-based detection scheme (b). In the fluorescence-based detection scheme, one of the $4 \mathrm{AJ}$ strands was augmented with a $10 \mathrm{nt}$ toehold to enable toehold-mediated strand invasion in order to remove of one of the dye-labeled strands. Upon removal of one of the dyes from the tetramer aggregate, excitonic quantum coherence between aggregates ceases and the dyes fluoresce. Samples were excited at $565 \mathrm{~nm}$ (the tetramer absorbance maximum) and monitored at $666 \mathrm{~nm}$ (the peak emission). The experiments were performed at room temperature with a DNA concentration of $\sim 0.4 \mu \mathrm{M}$. In the absorbance-based detection scheme, absorbance changes were induced by the combination of two halves of the 4AJ via DNA hybridization, thereby enabling tetramer formation and promoting absorbance at $565 \mathrm{~nm}$. The fluorescence-based experiments were performed at room temperature with a DNA concentration of $\sim 0.1 \mu \mathrm{M}$. The first 10 minutes of the data, which examines any baseline fluorescence/absorption due to the initial construct, has been removed along with small spikes that resulted from momentarily inserting the pipette into the incident light beam path.

Prior to (Figure 3.9; left panels) and subsequently following (Figure 3.9; right panels) the optical reporter experiments, static fluorescence (Figure 3.9; top panels) and absorbance (Figure 3.9; bottom panels) scans were obtained to ensure that correct aggregate formation or disassembly was occurring. 

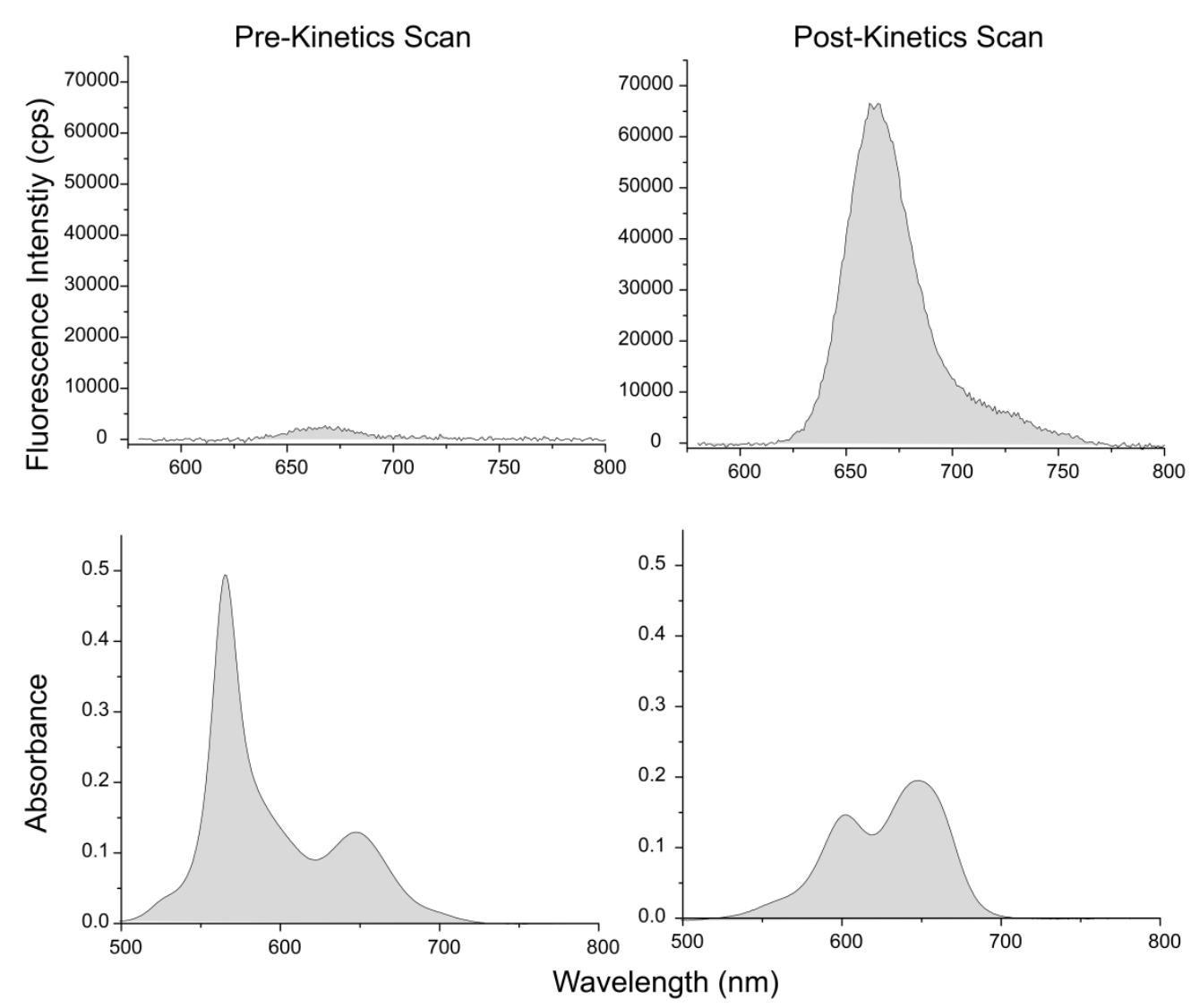

Figure 3.9. Absorbance (bottom panels) and fluorescence (top panels) spectra depicting the optical behavior of the $4 \mathrm{AJ}$ aggregate structures measured pre- (left panels, $t=0$ minutes) and post (right panels, $t=30$ minutes) reaction kinetic run as demonstrated in Figure 3.8a.

\section{$\underline{\text { 3.7.5 Structural Characterization }}$}

For structural characterization of the 4AJ-templated dye constructs, native polyacrylamide gel electrophoresis (PAGE) was used to separate structures by electrophoretic mobility to identify well-formed structures. Samples of DNA constructs at $10 \mu \mathrm{M}$ concentration with $15 \mathrm{mM}$ added $\mathrm{MgCl}_{2}$ were mixed in a 5:1 ratio with $6 \times \mathrm{New}$ England Biolabs loading buffer $(1 \times$ buffer: $11 \mathrm{mM}$ ethylenediaminetetraacetic acid, $3.3 \mathrm{mM}$ trishydrochloric acid, $0.017 \%$ sodium dodecyl sulfate, $0.015 \%$ bromophenol blue, $2.5 \%$ Ficoll@-400), injected into a 12\% native PAGE gel (10 cm length and $1.5 \mathrm{~mm}$ width), and allowed to run for approximately 120 minutes with a $150 \mathrm{~V}$ applied voltage. Staining 
agents were not used to avoid introducing external intercalants or dyes into the system. Rather, completed PAGE gel runs were placed onto a phosphor plate covered in plastic and imaged using UV illumination in a multiplexed gel imaging and documentation system (FluorChemQ, ProteinSimple). Gels were briefly illuminated with $254 \mathrm{~nm}$ UV light source and bands were identified by the shadows cast onto the illuminated phosphor plate. Bands containing the desired 4AJ-templated dye constructs were excised from the gels and crushed using a micro-mortar and pestle. Constructs were then extracted from the gel fragments by eluting in a $1 \times$ TAE buffer solution with $15 \mathrm{mM}$ added $\mathrm{MgCl}_{2}$.

Figure 3.10a shows an image of a completed gel run in which the 4AJ-templated dye constructs and the running buffer all contained $1 \times$ TAE $(40 \mathrm{mM}$ tris(hydroxymethyl)aminomethane, $20 \mathrm{mM}$ acetic acid, $1 \mathrm{mM}$ ethylenediaminetetraacetic acid; $\mathrm{pH}$ 8.0) buffer with $15 \mathrm{mM}$ added $\mathrm{MgCl}_{2}$. The PAGE gel was prepared with $0.25 \times$ TAE with $3.75 \mathrm{mM}$ added $\mathrm{MgCl}_{2}$. Labeled sample wells are located on the top of the gel image and DNA migration occurs from the top to bottom. The contents for each lane are illustrated at the top of the image. The first four lanes of the gel show the mobility of each single-stranded DNA through the gel and act as a control for the mobility of the larger, more complex structures. The middle two lanes (lane 5 and 6) indicate the two halves of the 4AJ (Dimer A and Dimer B) that were used for absorbance reaction kinetics studies. The right 4 lanes (lane 7-10) indicate 4AJ structures with various dye arrangements. It is evident from the gel that the mobility systematically increases with the increasing number of dye molecules. Whether this is due to the direct influence of the 
dyes on the mobility of due to changes in the geometry or stiffness of the $4 \mathrm{AJ}$ resulting from the incorporation of the dyes into the core is not known.

To confirm the stability of the tetramer structures in solution and verify that the constructs do no re-equilibrate in solution to form higher ordered structures, the yellow and orange bands of Lanes 9 and 10 were excised, the dye-DNA constructs eluted into a solution of buffer for 24 hours, and then re-purified in a second gel, as shown in Figure $3.10 \mathrm{~b}$ and Figure 3.10c, respectively, Note that the yellow- and orange-boxed lanes in Figure 3.10b and Figure 3.01c are samples representative of those excised from the yellow- and orange-boxed bands in Figure 3.10a. The first three lanes of Figure 3.10b, highlighted by a yellow box, shows a band where the higher ordered dye-DNA constructs are anticipated and a faint band indicating the presence of some $4 \mathrm{AJ}$ constructs. The second three lanes, highlighted in orange, only show a bright band where the 4AJ constructs are expected, and no band above indicating higher ordered DAN construct formation. Interestingly, Figure 3.10c shows no formation of higher ordered constructs in 
the first three lanes, highlighted in yellow, or the second three lanes, highlighted in orange.

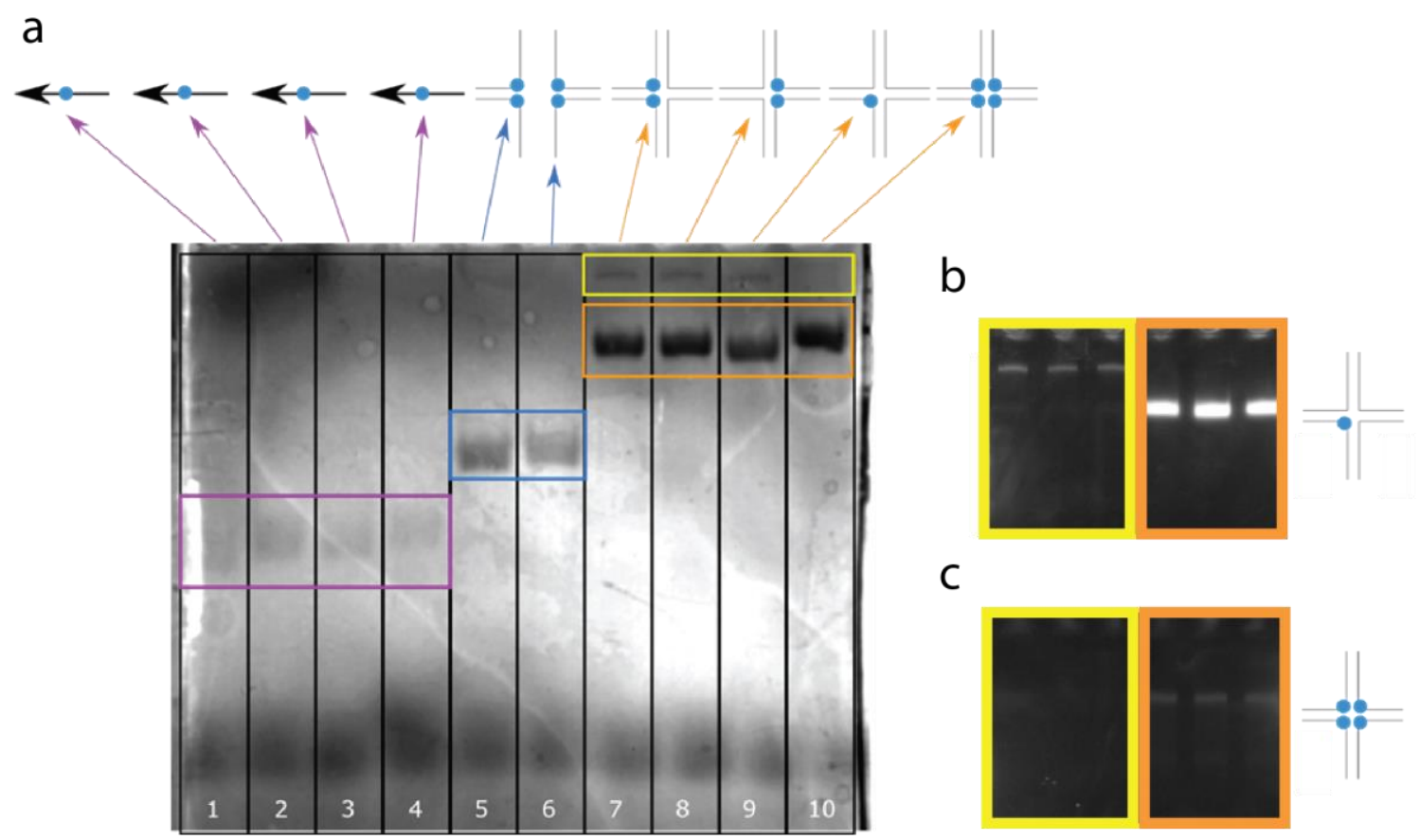

Figure 3.10. (a) Images of PAGE gel containing various 4AJ-templated dye-DNA constructs. All lanes that consist of DNA oligomers labeled with a single Cy5 dye molecule are identified by the violet box. Complexes that include two strands that are half-complementary and form a dye dimer are signified by the blue box. Orange boxes indicates well-formed $4 \mathrm{AJ}$ constructs with a variety of dye arrangements. The yellow box is used to highlight higher order DNA (most likely octamer) structures that form spontaneously in solution. Note that Lane 10, corresponding to the tetramer, does not have a band in the yellow-boxed region as compared to Lanes 7-9, indicating that higher ordered structures are not prevalent. Color-coded arrows direct towards the corresponding illustration of the DNA construct. To verify that the tetramer complexes were stable and did not form "octamer: structures while in equilibrium, the yellow and orange bands of (b) Lane 9 and (c) Lane 10 were excised, allowed to elute into solution and reach equilibrium over 24 hours, and then repurified in a PAGE gel.

\subsubsection{Salt and DNA Concentration Studies}

The PAGE gel in Figure 3.10 indicates that, apart from a small amount of octamer formation, the systems presented here exhibit no tendency to form higher ordered aggregates. Nevertheless, increased salt concentration and increased DNA concentration 
can favor the formation of higher ordered aggregates. To examine the stability against the formation of higher ordered aggregates and to investigate the possible dependence of the absorption spectrum on salt concentration, absorbance measurements were performed as a function of salt and DNA concentration. Figure 3.11 demonstrates the stability of the 4AJbased $\mathrm{H}$-tetramer in solution with varying salt (i.e., $\mathrm{MgCl}_{2}$ ) and DNA concentrations. Note, that the dye concentration scales linearly with the DNA concentration. The left panel of Figure S6.1 shows H-tetramer stability within a wide range of salt concentration, 0-100 $\mathrm{mM} \mathrm{MgCl} 2$, as depicted by the absorbance peak at $565 \mathrm{~nm}$. Samples were held constant at 1.5 $\mu \mathrm{M}$ DNA concentration and $22{ }^{\circ} \mathrm{C}$ temperature. Similarly, in the right panel of Figure S6.1, the absorbance peak at $565 \mathrm{~nm}$, indicative of an H-tetramer, is observed at all DNA concentrations (0.1-3.0 $\mu \mathrm{M})$. Samples were held constant at $15 \mathrm{mM} \mathrm{MgCl}_{2}$ and $22{ }^{\circ} \mathrm{C}$.

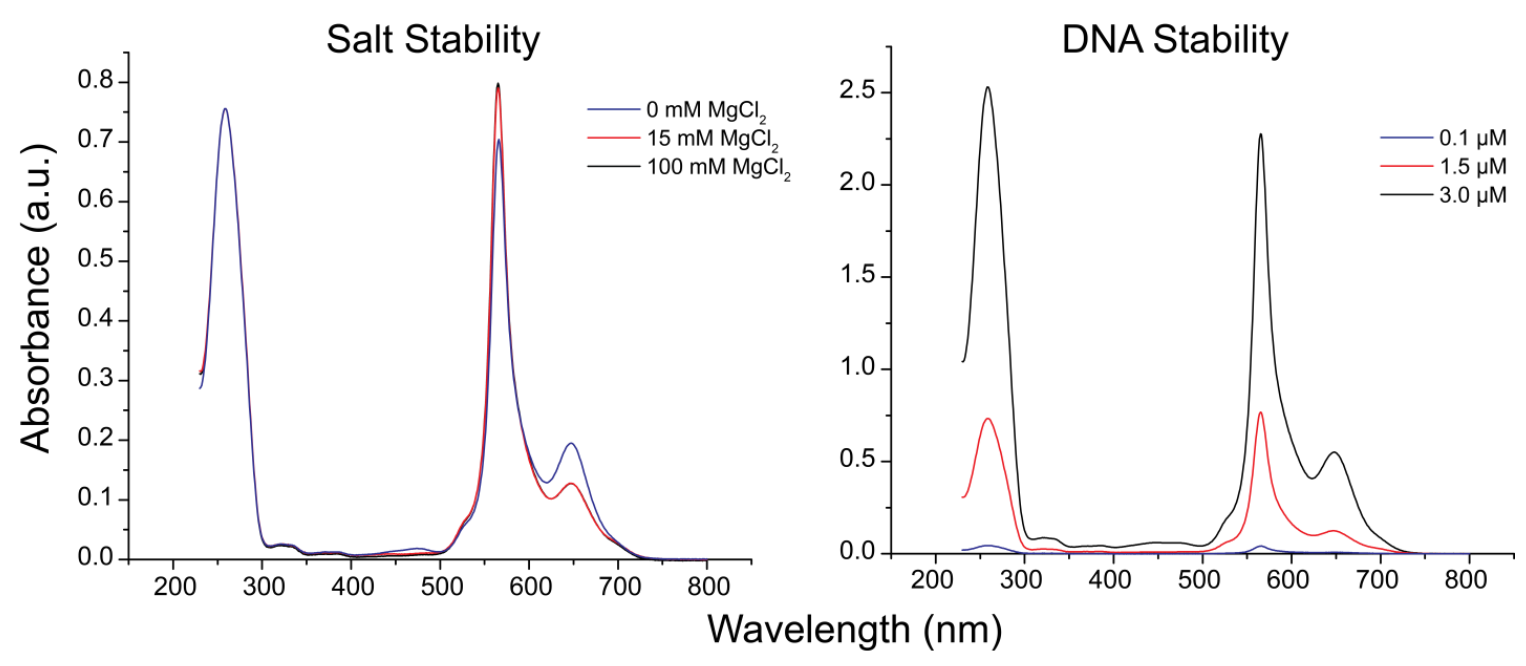

Figure 3.11. UV-Vis absorbance spectra demonstrating the stability of the $4 \mathrm{AJ}$ tetramer in solution with variable salt (i.e., $\mathrm{MgCl}_{2}$, left panel) and DNA (right panel) concentrations. The salt stability experiment was conducted with samples held at constant DNA concentrations of $1.5 \mu \mathrm{M}$, while DNA stability samples were conducted with samples held at constant salt concentrations of $15 \mathrm{mM} \mathrm{MgCl}$. 


\section{REFERENCES}

[1] Frenkel, J., On the Transformation of Light into Heat in Solids. II. Physical Reviews 1931, 37 (10), 1276-1294.

[2] Davydov, A. S., Theory of Absorption Spectra of Molecular Crystals. Institute of Physics, Academy of Sciences of Ukrainian SSR: Kyiv, Ukraine, 1948; 18.

[3] Davydov, A. S., The Theory of Molecular Excitons. Soviet Physics 1964, 82 (3-4), 145-178.

[4] Kasha, M., Energy Transfer Mechanisms and Molecular Exciton Model for Molecular Aggregates. Radiation Research 1963, 20 (1), 55.

[5] Kasha, M., The Exciton Model in Molecular Spectroscopy. Pure and Applied Chemistry 1965, 11 (3-4), 371.

[6] Franck, J.; Teller, E., Migration and Photochemical Action of Excitation Energy in Crystals. Journal of Chemical Physics 1938, 6 (12), 861-872.

[7] Eisfeld, A.; Briggs, J. S., The J-Band of Organic Dyes: Lineshape and Coherence Length. Chemical Physics 2002, 281 (1), 61-70.

[8] Fidder, H.; Knoester, J.; Wiersma, D. A., Optical Properties of Disordered Molecular Aggregates: A Numerical Study. Journal of the American Chemical Society, 1991, 95 (11), 7880-7890.

[9] Roden, J.; Eisfeld, A.; Briggs, J. S., The J- and H-bands of Dye Aggregate Spectra: Analysis of the Coherent Exciton Scattering (CES) Approximation. Chemical Physics 2008, 352 (1-3), 258-266.

[10] Eisfeld, A.; Briggs, J. S., Absorption Spectra of Quantum Aggregates Interacting via Long-Range Forces. Physcial Review Letters 2006, 96 (11).

[11] Whittaker, D. M.; Kinsler, P.; Fisher, T. A.; Skolnick, M. S.; Armitage, A.; Afshar, A. M.; Sturge, M. D.; Roberts, J. S., Motional Narrowing in Semiconductor Microcavities. Physical Review Letters 1996, 77 (23), 4792-4795. 
[12] Walczak, P. B.; Eisfeld, A.; Briggs, J. S., Exchange Narrowing of the J Band of Molecular Dye Aggregates. Journal of Chemical Physics 2008, 128 (4).

[13] Knapp, E. W., Lineshapes of Molecule Aggregates - Exchange Narrowing and Intersite Correlation. Chemical Physics 1984, 85 (1), 73-82.

[14] Marcus, R. J.; Haugen, G. R., Resonance Fluorescence in Chlorophyll a Solutions. Photochemistry and Photobiology 1965, 4, 183-192.

[15] Ogawa, M.; Kosaka, N.; Choyke, P. L.; Kobayashi, H., H-Type Dimer Formation of Fluorophores: A Mechanism for Activatable, in Vivo Optical Molecular Imaging. ACS Chemical Biology 2009, 4 (7), 535-546.

[16] Ikeda, S.; Kubota, T.; Kino, K.; Okamoto, A., Sequence Dependence of Fluorescence Emission and Quenching of Doubly Thiazole Orange Labeled DNA: Effective Design of a Hybridization-Sensitive Probe. Bioconjugate Chemistry 2008, 19 (8), 1719-1725.

[17] Ikeda, S.; Okamoto, A., Hybridization-Sensitive On-Off DNA Probe: Application of the Exciton Coupling Effect to Effective Fluorescence Quenching. Chemistry - An Asian Journal 2008, 3 (6), 958-968.

[18] Hara, Y.; Fujii, T.; Kashida, H.; Sekiguchi, K.; Liang, X. G.; Niwa, K.; Takase, T.; Yoshida, Y.; Asanuma, H., Coherent Quenching of a Fluorophore for the Design of a Highly Sensitive In-Stem Molecular Beacon. Angewandte Chemie - International Edition 2010, 49 (32), 5502-5506.

[19] Bouquin, N.; Malinovskii, V. L.; Haener, R., Highly Efficient Quenching of Excimer Fluorescence by Perylene Diimide in DNA. Chemical Communications 2008, (17), 1974-1976.

[20] Eisfeld, A.; Kniprath, R.; Briggs, J. S., Theory of the Absorption and Circular Dichroism Spectra of Helical Molecular Aggregates. Journal of Chemical Physics 2007, 126 (10).

[21] Person, R. V.; Monde, K.; Humpf, H. U.; Berova, N.; Nakanishi, K., A New Approach in Exciton-Coupled Circular-Dichroism (ECCD) - Insertion of an Auxillary Stereogenic Center. Chirality 1995, 7 (3), 128-135.

[22] Ruedas-Rama, M. J.; Orte, A.; Martin-Domingo, M. C.; Castello, F.; Talavera, E. M.; Alvarez-Pez, J. M., Interaction of YOYO-3 with Different DNA Templates to Form H-Aggregates. Journal of Physical Chemistry B 2014, 118 (23), 6098-6106. 
[23] Ruedas-Rama, M. J.; Alvarez-Pez, J. M.; Orte, A., Formation of Stable BOBO-3 HAggregate Complexes Hinders DNA Hybridization. Journal of Physical Chemistry B 2010, 114 (27), 9063-9071.

[24] Asanuma, H.; Fujii, T.; Kato, T.; Kashida, H., Coherent Interactions of Dyes Assembled on DNA. Journal of Photochemistry and Photobiology C 2012, 13 (2), 124-135.

[25] Asanuma, H.; Shirasuka, K.; Takarada, T.; Kashida, H.; Komiyama, M., DNA-Dye Conjugates for Controllable $\mathrm{H}^{*}$ Aggregation. Journal of the American Chemical Society 2003, 125 (8), 2217-2223.

[26] Yang, M. N., Influence of Energy Transfer on the Intensity Pattern of Vibronic Excitation Studied by Reduced Density-Matrix Theory. The Jounral of Molecular Spectroscopy 2006, 239 (1), 108-114.

[27] Markova, L. I.; Malinovskii, V. L.; Patsenker, L. D.; Haner, R., J- vs. H-Type Assembly: Pentamethine Cyanine (Cy5) as a Near-IR Chiroptical Reporter. Chemical Communications 2013, 49 (46), 5298-5300.

[28] Conley, N. R.; Pomerantz, A. K.; Wang, H.; Twieg, R. J.; Moerner, W. E., Bulk and Single-Molecule Characterization of an Improved Molecular Beacon Utilizing $\mathrm{H}-$ Dimer Excitonic Behavior. Journal of Physical Chemistry B 2007, 111 (28), 79297931.

[29] Lim, J. M.; Kim, P.; Yoon, M. C.; Sung, J.; Dehm, V.; Chen, Z. J.; Wurthner, F.; Kim, D., Exciton Delocalization and Dynamics in Helical Pi-Stacks of Self-Assembled Perylene Bisimides. Chemical Science 2013, 4 (1), 388-397.

[30] Ikeda, S.; Kubota, T.; Yuki, M.; Okamoto, A., Exciton-Controlled HybridizationSensitive Fluorescent Probes: Multicolor Detection of Nucleic Acids. Angewandte Chemie - International Edition 2009, 48 (35), 6480-6484.

[31] Jelley, E., E., Molecular, Nematic and Crystal States of I: I-Diethyl--Cyanine Chloride. Nature 1937, 139.3519, 631-632.

[32] Jelley, E. E., Molecular, Nematic and Crystal States of I: I-Diethyl-Cyanine Chloride. Nature 1937, 139 (3519), 631-632.

[33] Scheibe, G., Über die Veränderlichkeit der Absorptionsspektren in Lösungen und die Nebenvalenzen als ihre Ursache. Angewandte Chemie 1937, 50.11, 212-219. 
[34] Scheibe, G.; Kandler, L.; Ecker, H., Polymerisation and Polymere Adsorption as a Cause of Novel Absorption Bands of Organic Pigments. Naturwissenschaften 1937, $25,75-75$.

[35] Scheibe, G.; Mareis, A.; Ecker, H., The Reversible Polymerisation as a Cause of Unusual Absorption Bands III. Naturwissenschaften 1937, 25, 474-475.

[36] Stiel, H.; Teuchner, K.; Becker, W.; Freyer, W.; Dahne, S., Flurescence Lifetime Studies of Pseudoisocyaniny J-Aggregates in the Subnanosecond Range. The Journal of Molecular Structure 1984, 114, 351-354.

[37] Fidder, H.; Knoester, J.; Wiersma, D. A., Superradiant Emission and Optical Dephasing in J-aggregates. Chemical Physical Letters 1990, 171 (5-6), 529-536.

[38] Eisfeld, A.; Briggs, J. S., The J- and H-Bands of Organic Dye Aggregates. Chemical Physics 2006, 324 (2-3), 376-384.

[39] Eisfeld, A.; Briggs, J. S., The Shape of the J-Band of Pseudoisocyanine. Chemical Physics Letters 2007, 446 (4-6), 354-358.

[40] Spano, F. C., The Spectral Signatures of Frenkel Polarons in H- and J-Aggregates. Account of Chemical Research 2010, 43 (3), 429-439.

[41] Wuerthner, F.; Kaiser, T. E.; Saha-Moeller, C. R., J-Aggregates: From Serendipitous Discovery to Supramolecular Engineering of Functional Dye Materials. Angewandte Chemie - International Edition 2011, 50 (15), 3376-3410.

[42] Li, Z. a.; Mukhopadhyay, S.; Jang, S.-H.; Bredas, J.-L.; Jen, A. K. Y., Supramolecular Assembly of Complementary Cyanine Salt J-Aggregates. Journal of the American Chemical Society 2015, 137 (37), 11920-11923.

[43] Pajusalu, M.; Raetsep, M.; Trinkunas, G.; Freiberg, A., Davydov Splitting of Excitons in Cyclic Bacteriochlorophyll a Nanoaggregates of Bacterial Light-Harvesting Complexes between 4.5 and 263 K. Chemphyschem 2011, 12 (3), 634-644.

[44] von Berlepsch, H.; Bottcher, C., Supramolecular Structure of TTBC J-Aggregates in Solution and on Surface. Langmuir 2013, 29 (16), 4948-4958.

[45] Monshouwer, R.; Abrahamsson, M.; vanMourik, F.; vanGrondelle, R., Superradiance and Exciton Delocalization in Bacterial Photosynthetic Light-Harvesting Systems. Journal of Physical Chemistry B 1997, 101 (37), 7241-7248. 
[46] Dahlbom, M.; Pullerits, T.; Mukamel, S.; Sandstrom, V., Exciton Delocalization in the B850 Light-Harvesting Complex: Comparison of Different Measures. Journal of Physical Chemistry B 2001, 105 (23), 5515-5524.

[47] Scholes, G. D.; Rumbles, G., Excitons in Nanoscale Systems. Nature Materials 2006, 5 (9), 683-696.

[48] Blancafort, L.; Voityuk, A. A., Exciton Delocalization, Charge Transfer, and Electronic Coupling for Singlet Excitation Energy Transfer Between Stacked Nucleobases in DNA: An MS-CASPT2 Study. The Journal of Chemical Physics 2014, $140(9)$.

[49] Scholes, G. D.; Ghiggino, K. P.; Oliver, A. M.; Paddonrow, M. N., Through-Space and Through-Bond Effects on Exciton Interactions in Rigidly Linked Dinaphthyl Molecules. Journal of the American Chemical Society 1993, 115 (10), 4345-4349.

[50] Saikin, S. K.; Eisfeld, A.; Valleau, S.; Aspuru-Guzik, A., Photonics Meets Excitonics: Natural and Artificial Molecular Aggregates. Nanophotonics 2013, 2 (1), 21-38.

[51] Schroter, M.; Ivanov, S. D.; Schulze, J.; Polyutov, S. P.; Yan, Y.; Pullerits, T.; Kühn, O., Exciton-Vibrational Coupling in the Dynamics and Spectroscopy of Frenkel Excitons in Molecular Aggregates. Physical Reports 2015, 567, 1-78.

[52] Probst, M.; Langenegger, S. M.; Haner, R., A Modular LHC Built on the DNA ThreeWay Junction. Chemical Communications 2014, 50 (2), 159-161.

[53] Markova, L. I.; Malinovskii, V. L.; Patsenker, L. D.; Haner, R., Synthesis and Properties of Squaraine-Modified DNA. Organic \& Biomolecular Chemistry 2012, 10 (45), 8944-8947.

[54] Malinovskii, V. L.; Wenger, D.; Haner, R., Nucleic Acid-Guided Assembly of Aromatic Chromophores. Chemical Society Reviews 2010, 39 (2), 410-422.

[55] Li, S. G.; Langenegger, S. M.; Haner, R., Control of Aggregation-Induced Emission by DNA Hybridization. Chemical Communications 2013, 49 (52), 5835-5837.

[56] Haner, R.; Samain, F.; Malinovskii, V. L., DNA-Assisted Self-Assembly of Pyrene Foldamers. Chemistry - A European Journal 2009, 15 (23), 5701-5708.

[57] Garo, F.; Haner, R., A DNA-Based Light-Harvesting Antenna. Angewandte Chemie International Edition 2012, 51 (4), 916-919. 
[58] Adeyemi, O. O.; Malinovskii, V. L.; Biner, S. M.; Calzaferri, G.; Haner, R., Photon Harvesting by Excimer-Forming Multichromophores. Chemical Communications 2012, 48 (77), 9589-9591.

[59] Kashida, H.; Asanuma, H., Preparation of Supramolecular Chromophoric Assemblies using a DNA Duplex. Physical Chemistry Chemical Physics 2012, 14 (20), 71967204.

[60] Wei, B.; Dai, M. J.; Yin, P., Complex Shapes Self-Assembled from Single-Stranded DNA Tiles. Nature 2012, 485 (7400), 623-626.

[61] Rothemund, P. W. K.; Andersen, E. S., Nanotechnology: The Importance of Being Modular. Nature 2012, 485 (7400), 584-585.

[62] Ke, Y. G.; Ong, L. L.; Shih, W. M.; Yin, P., Three-Dimensional Structures SelfAssembled from DNA Bricks. Science 2012, 338 (6111), 1177-1183.

[63] Wang, M. M.; Silva, G. L.; Armitage, B. A., DNA-Templated Formation of a Helical Cyanine Dye J-Aggregate. Journal of the American Chemical Society 2000, 122 (41), 9977-9986.

[64] Kashida, H.; Asanuma, H.; Komiyama, M., Alternating Hetero H Aggregation of Different Dyes by Interstrand Stacking from Two DNA-Dye Conjugates. Angewandte Chemie - International Edition 2004, 43 (47), 6522-6525.

[65] Kashida, H.; Tanaka, M.; Baba, S.; Sakamoto, T.; Kawai, G.; Asanuma, H.; Komiyama, M., Covalent Incorporation of Methyl Red Dyes into Double-Stranded DNA for Their Ordered Clustering. Chemistry - A European Journal 2006, 12 (3), 777-784.

[66] Fujii, T.; Kashida, H.; Asanuma, H., Analysis of Coherent Heteroclustering of Different Dyes by Use of Threoninol Nucleotides for Comparison with the Molecular Exciton Theory. Chemistry - A European Journal 2009, 15 (39), 10092-10102.

[67] Probst, M.; Wenger, D.; Biner, S. M.; Haner, R., The DNA Three-Way Junction as a Mould for Tripartite Chromophore Assembly. Organic \& Biomolecular Chemistry 2012, 10 (4), 755-759.

[68] Cannon, B. L.; Kellis, D. L.; Patten, L. K.; Davis, P. H.; Lee, J.; Graugnard, E.; Yurke, B.; Knowlton, W. B., Coherent Exciton Delocalization in a Two-State DNATemplated Dye Aggregate System. Journal of Physical Chemistry A 2017, 121 (37), 6905-6916. 
[69] Seeman, N. C., Nucleic-Acid Junctions and Lattices. The Journal of Theoretical Biology, 1982, 99, 237-247.

[70] Kallenbach, N. R.; Ma, R.-I.; Seeman, N. C., An Immobile Nucleic-Acid Junction Contstructed from Oligonucleotides. Nature, 1983, 305, 829-831.

[71] Tomita, G., Absorption and Fluorescence Properties of Some Basic Dyes Complexing with Nucleosides or Nucleic Acids. Z. Naturforsch. Pt. B 1968, B 23 (7), 922.

[72] Kühn, O.; Renger, T.; May, V., Theory of Exciton-Vibrational Dynamics in Molecular Dimers. Chemical Physics 1996, 204 (1), 99-114.

[73] Pettersen, E. F.; Goddard, T. D.; Huang, C. C.; Couch, G. S.; Greenblatt, D. M.; Meng, E. C.; Ferrin, T. E., UCSF chimera - A Visualization System for Exploratory Research and Analysis. The Journal of Computational Chemistry 2004, 25 (13), 1605-1612.

[74] Couch, G. S.; Hendrix, D. K.; Ferrin, T. E., Nucleic Acid Visualization with UCSF Chimera. Nucleic Acids Research 2006, 34 (4).

[75] Yurke, B.; Turberfield, A. J.; Mills, A. P.; Simmel, F. C.; Neumann, J. L., A DNAFuelled Molecular Machine Made of DNA. Nature 2000, 406 (6796), 605-608.

[76] Srinivas, N.; Ouldridge, T. E.; Sulc, P.; Schaeffer, J. M.; Yurke, B.; Louis, A. A.; Doye, J. P. K.; Winfree, E., On the Biophysics and Kinetics of Toehold-Mediated DNA Strand Displacement. Nucleic Acids Research 2013, 41 (22), 10641-10658. 


\section{CHAPTER FOUR: SUMMARY AND FUTURE WORK}

In conclusion, exciton delocalization has been observed and explored in two DNA-templated systems: (1) a simple DNA duplex forming a Cy5 dimer aggregate that transitions to a mobile four-armed junction creating a tetramer upon increases in salt and/or DNA concentrations and (2) an immobile Holliday junction used to form dye aggregates of pre-determined size including two different dimer arrangements (i.e., adjacent and transverse), a trimer, and a tetramer. Both studies have provided foundational information that can be built upon for the development of higher-ordered aggregate systems to form arrays and networks for potential applications in lightharvesting and/or quantum information processing.

Fundamental observations of coherent exciton delocalization in an immobile Holliday Junction are listed below (Chapter 2):

1. Constructing a DNA duplex with two spatially proximate Cy5 dyes on opposing strands at a low concentration of salt and DNA resulted in the formation of a J-dimer as demonstrated by absorbance spectra showing a slightly red-shifted absorbance peak $(665 \mathrm{~nm})$ relative to the monomer peak $(645 \mathrm{~nm})$. Increasing the salt and/or DNA concentration of the solution facilitated the hybridization of two duplexes via a strandmediated displacement reaction to form a mobile four-armed junction (4AJ) construct, thus creating a tetramer. Due to the mobility of the $4 \mathrm{AJ}$ that occurred from DNA breathing and base pair restacking at the junction 
core, two aggregate populations existed simultaneously: (i) a tetramer with predominantly $\mathrm{H}$-aggregate-like behavior that was identified by a significantly blue-shifted absorbance peak $(562 \mathrm{~nm})$ relative to the monomer, and (ii) dimers that demonstrate predominantly J-aggregate like behavior with a red-shifted absorbance peak $(665 \mathrm{~nm})$ compared to the monomer and small Stokes shift (2 nm).

2. In low salt and DNA concentration solutions, the J-aggregate displayed near resonant fluorescence ( $2 \mathrm{~nm}$ Stokes shift relative to the absorbance maximum). Conversely, the H-aggregate (tetramer) showed a large Stokes shift of $104 \mathrm{~nm}$ and significantly decreased fluorescence. The observation of a CD signal for both the J- and H-type aggregates indicates some oblique stacking, in line with the observed Davydov splitting.

3. Changing the position of one of the dyes by a single base-pair led to the complete disappearance of the blue-shifted peak. Further increasing the separation distance between the dyes to two base-pairs extinguished all spectral signatures of exciton delocalization, demonstrating a strong distance dependence.

4. The observed Davydov splitting of $103 \mathrm{~nm}$ (or $336 \mathrm{meV}$ ) is significantly larger than that seen in other DNA-templated aggregate schemes with $\sim 200$ meV splitting. ${ }^{67}$

5. The mechanism driving the transition between the duplex and $4 \mathrm{AJ}$ structures was highly dependent on both the external salt concentration 
and the effective DNA (i.e., dye) concentration, which was modeled by a second-order reaction kinetics mechanism equation.

6. The switching between the two different discrete states with a resultant change in the equilibrium of the two species was initially hypothesized to be composed of multiple (2+) duplexes, and thus dye-dimers. Ultimately it was determined that two-dye dimer formed a single tetramer. Further characterization involving time-dependent reaction kinetics and gelelectrophoresis emphasized this hypothesis.

7. A phenomenological theoretical model (KRM model) was used and physical parameters were defined based on spectral data.

Building upon the duplex study, an immobile four-armed DNA structure was constructed with zero to $4 \mathrm{Cy} 5$ dyes positioned in the center of the junction. The concluding observations for this study are listed here (Chapter 3):

1. Depending on the number and relative position(s) of dyes present in the system, drastically different optical properties were observed.

2. The Davydov splitting exhibited by the immobile 4AJ-templated tetramer aggregate (135 $\mathrm{nm}$ or $423 \mathrm{meV}$ ) was found to be larger than that observed in the duplex-templated mobile 4AJ tetramer aggregate (103 nm or 336 meV). The splitting, originally determined by performing peak analysis on the absorbance spectrum, was validated by the observation of two large CD peaks corresponding to the blue-shifted absorbance peak at $565 \mathrm{~nm}$ and the red-shifted peak at $700 \mathrm{~nm}$. 
3. Using the simple design rules afforded by the DNA scaffold and exploiting the unique optical properties provided by the 4AJ-templated tetramer, two optical detection schemes were demonstrated using DNA strand-invasion. The first exploited the strong fluorescence suppression of the tetramer to demonstrate a sensitive fluorescence detector and the second used the large Davydov splitting observed in the tetramer to fabricate a colorimetric detection system.

4. The fabrication of a Cy5 tetramer aggregate that exhibits large Davydov splitting establishes the core necessary for an excitonic beam splitter. ${ }^{82}$ The construction of such devices could lead to the development of a quantum computer that operates at room temperature.

5. Validation of the KRM model wherein it was determined that the same physical parameters used in Chapter 2, could be applied to other dye configurations.

Overall, this work provides a substantial foundation and contributes encouraging results for the continued investigation into coherent exciton delocalization in DNAtemplated dye aggregate systems, specifically with applications towards quantum computing. A few directions that could be explored for future studies include:

1. Development of devices, including beam splitters and phase shifters, to construct and demonstrate the basic gates necessary for universal quantum computing. An example of how this may be executed is illustrated in Figure 4.1. As described by Yurke and Kuang, ${ }^{82}$ an excitonic beam splitter can be fabricated by assembling molecular dyes on a scaffold in a manner such that 
excitonic energy transfer is directed down one arm (in), travels towards the core of the structure, which then splits the possible path of the exciton down two or more spatially independent arms (i.e., out 1 , out $2, \ldots$ out $n$ ). As preliminarily presented in Chapter 3 and illustrate in Figure 4.1, the core of the beam splitter can be realized with molecular dyes that exhibit exciton delocalization and are attached at the center of the four arms of a junction. Specifically, selected dyes could then be attached along the length of the arms to act as an energy transfer guide or pathway by utilizing a combination of exciton delocalization and FRET mechanisms. Thus, the final readout of the system would be purely optical, with the output of one arm indicated by one color/wavelength (e.g., green), and the output of the second arm indicated by a different color/wavelength (e.g., yellow).
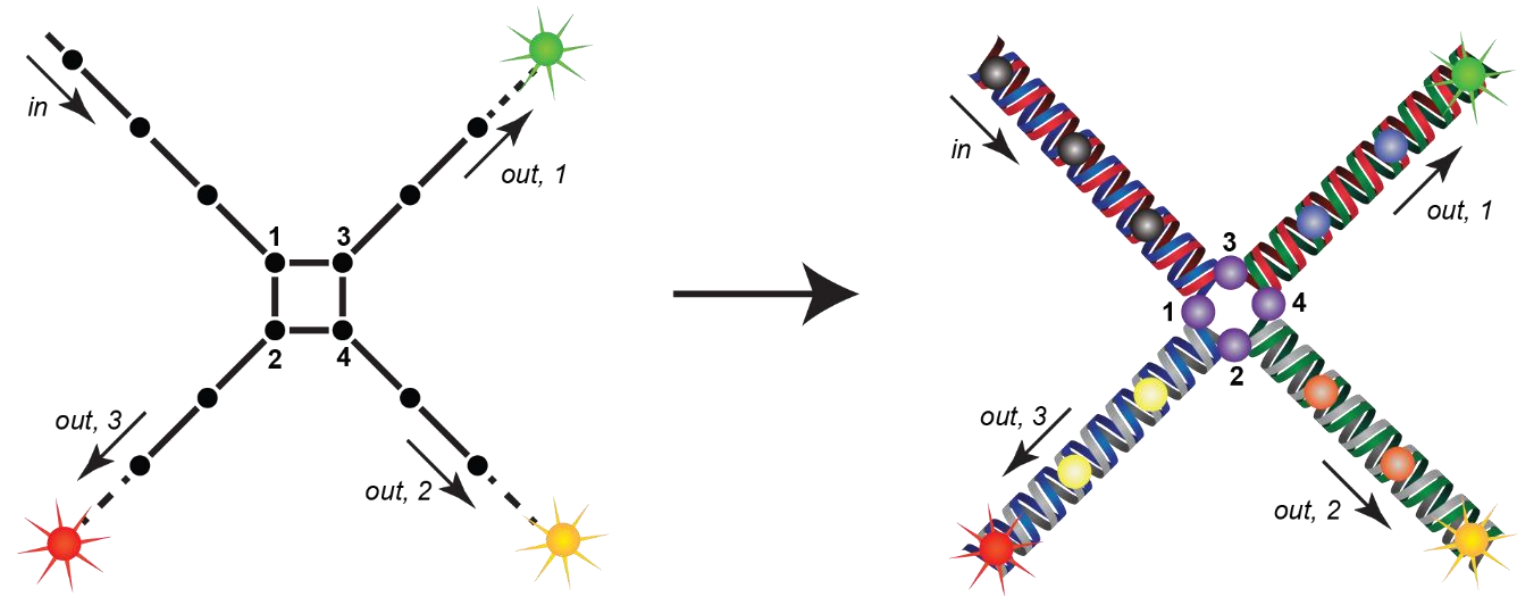

Figure 4.1. Illustration of an excitonic beam splitter with optical readouts. Adapted by Yurke and Kuang. ${ }^{82}$

2. Demonstration of exciton delocalization between dye aggregates assembled on a rigid DNA 'nanobreadboard'. Essentially, the DNA nanobreadboard would act as a structural platform for assembling molecular dyes into specific 
nanoscale patterns and arrays. The advantage of the DNA nanobreadboard, as opposed to a duplex or four-armed junction, is the superior rigidity offered by the complex structure and the ability to modularly assemble large molecular circuits and arrays.

3. Investigations into alternative dye types amenable to configuring into dye aggregates by identifying molecular structures (i.e., symmetric or asymmetric), sizes, and types to better promote Davydov splitting (i.e., greater coupling between the molecules).

4. Use of the KRM theoretical model as a predictive engineering tool for designing excitonic devices and quantum gates and as an analysis tool for determining dye aggregate configurations. 